\title{
Environmental Support FY 1995 Multi-Year Program Plan/Fiscal Year Work Plan WBS 1.5.2/7.4.11
}

\author{
D. A. Moore
}

Date Published

September 1994

Prepared for the U.S. Department of Energy Office of Environmental Restoration and Waste Management
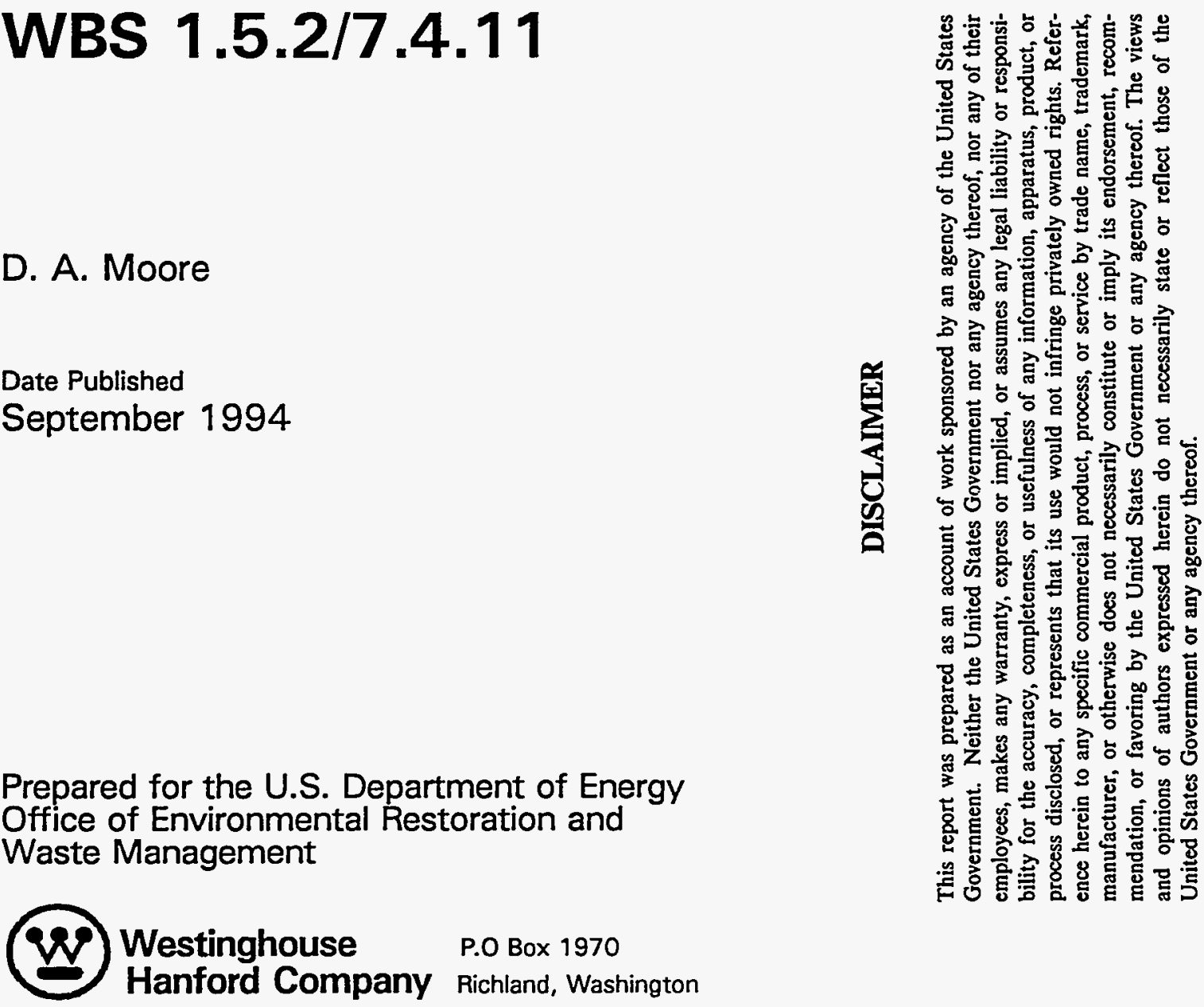

Hanford Operations and Engineering Contractor for the

U.S. Department of Energy under Contract DE-AC06-87RL10930

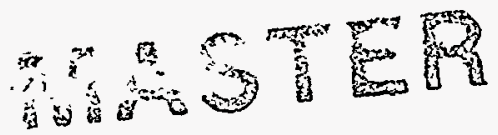

Approved for Public Release 


\section{LEGAL DISCLAIMER}

This report was prepared as an account of work sponsored by an agency of the United States Government. Neither the United States Government nor any agency thereof, nor any of their employees, nor any of their contractors, subcontractors or their employees, makes any warranty, express or implied, or assumes any legal liability or responsibility for the accuracy, completeness, or any third party's use or the results of such use of any information, apparatus, product, or process disclosed, or represents that its use would not infringe privately owned rights. Reference herein to any specific commercial product, process, or service by trade name, trademark, manulacturer, or otherwise, does not necessarily constitute or imply its endorsement, recommendation, or favoring by the United States Government or any agency thereof or its contractors or subcontractors. The views and opinions of authors expressed herein do not necessarily state or reflect those of the United States Government or any agency thereof.

This report has been reproduced from the best available copy. Available in paper copy and microfiche.

Available to the U.S. Department of Energy

and its contractors from

Office of Scientific and Technical Information

P O. Box 62

Oak Ridge, TN 37831

(615) 576-8401

Available to the public from the U.S. Department of Commerce National Technical Information Service

5285 Port Royal Road

Springfield, VA 22161

(703) $487-4650$

Punted in the Uniled States of Amenca

DISCLM-1 CHP (1-91) 


\section{DISCLAIMER}

Portions of this document may be illegible in electronic image products. Images are produced from the best available original document. 
WHC-SP-1112

DISTRIBUTION

Number of copies

OFFSITE

5 Department of Energy-Richland Operations Office

E. B. Dagan

A5-15

R. G. Holt

A5-15

C. R. Pasternak

G3-18

(2) W. A. Rutherford

A5-04

\section{ONSITE}

25 Westinghouse Hanford Company

P. J. Bence

G1-76

B. L. Bloom

G1-50

D. L. Borders

B3-62

(7) P. A. Callender

H6-30

G. D. Carpenter

H6-21

W. T. Dixon

H6-21

(2)

D. M. Eder

B3-62

J. K. Fish

B3-62

J. T. Funk

B1-61

C. T. Hewitt

B3-58

D. A. Moore

H6-21

L. L. Powers

H6-28

J. A. Sargent

B3-75

(2) O.S.T.I

L8-07

(2) Central Files

L8-04

EPIC

H6-08 


\subsubsection{Environmental Support}

U. S. Department of Energy

Richland Operations office

\section{Assistant Manager-Contracting Officer's Representative}
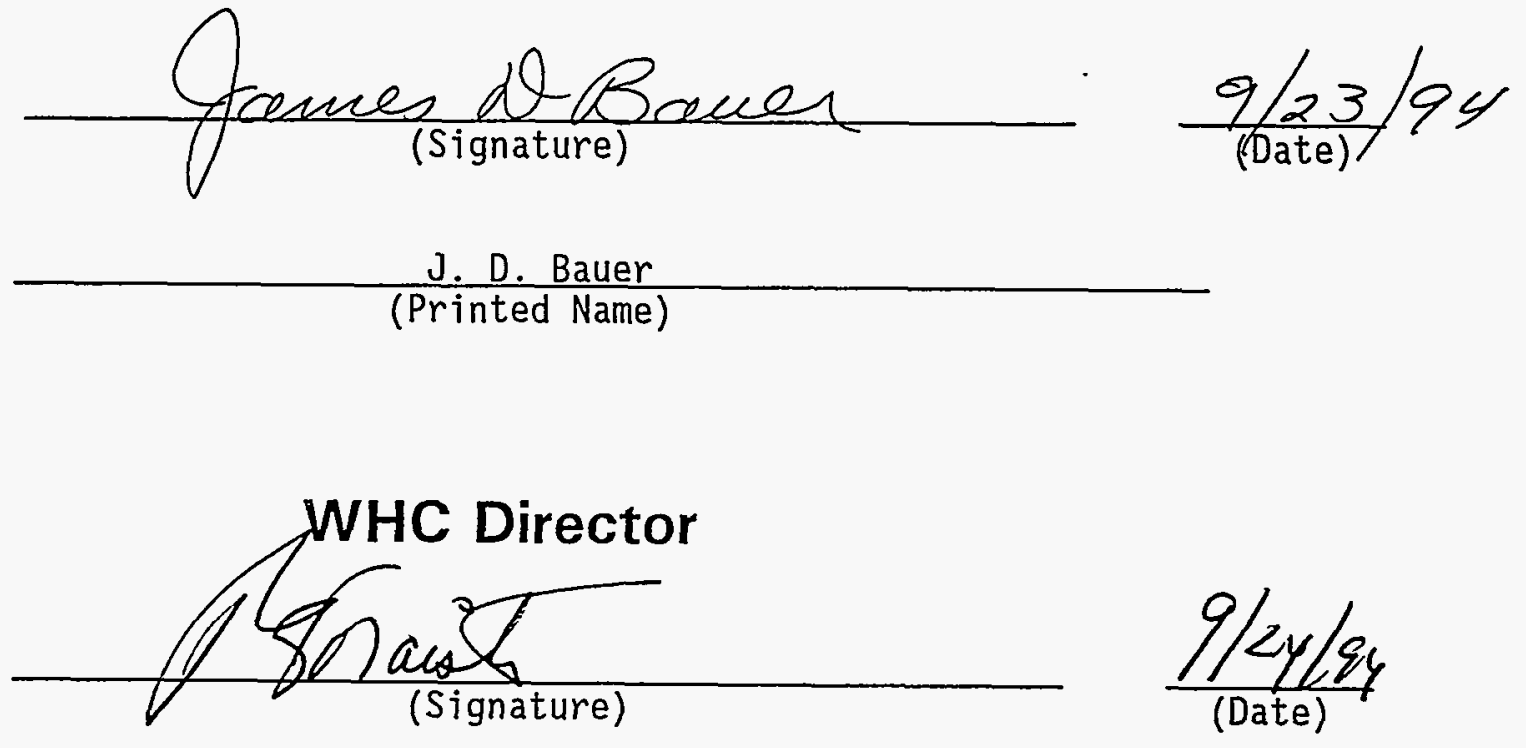

R. E. Traister (Printed Name) 


\subsubsection{Environmental Support}

U. S. Department of Energy

Richland Operations office

RL Program Manager(s)

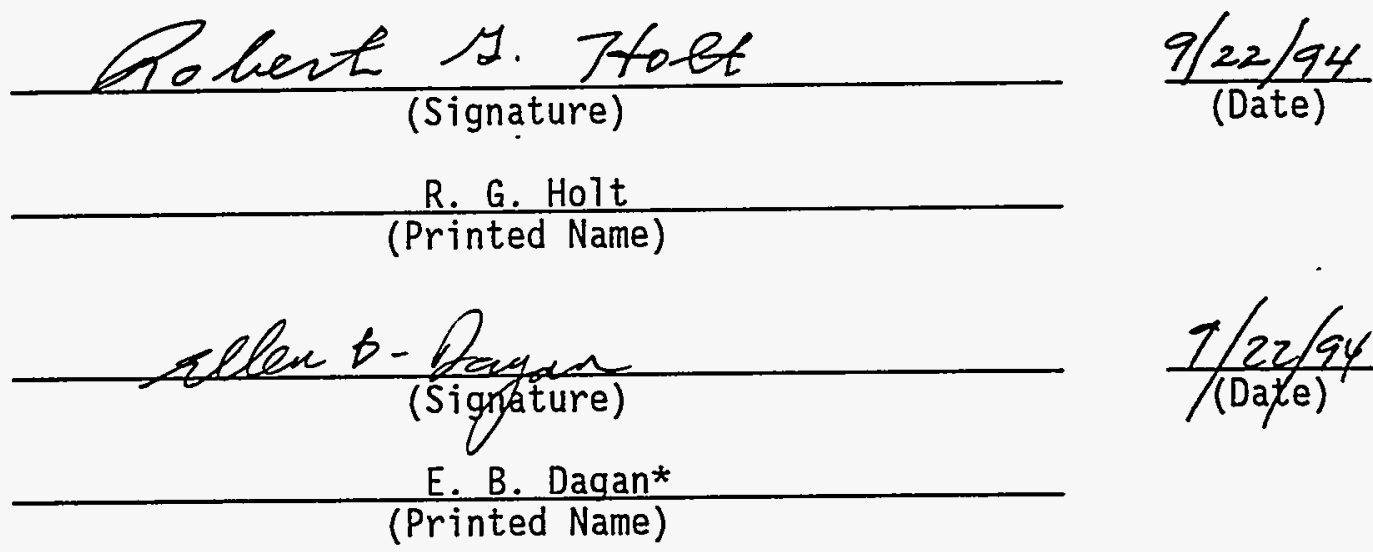

\section{WHC Manager}
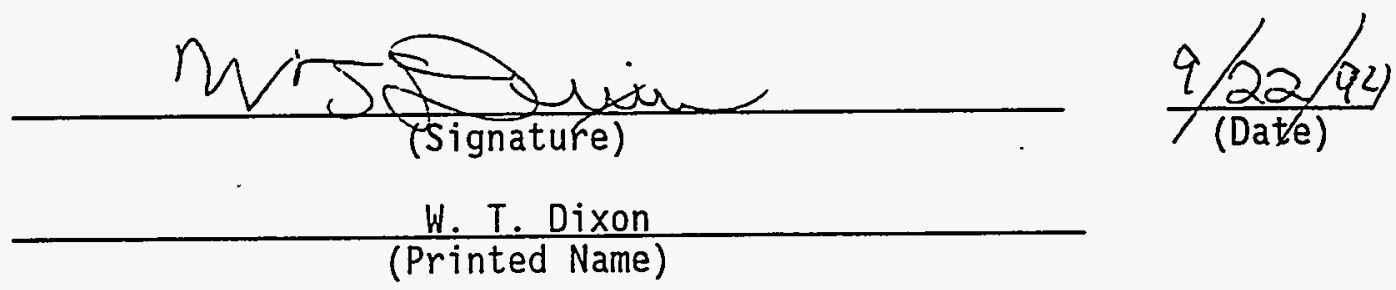

(Printed Name)

\section{WHC Program Manager}

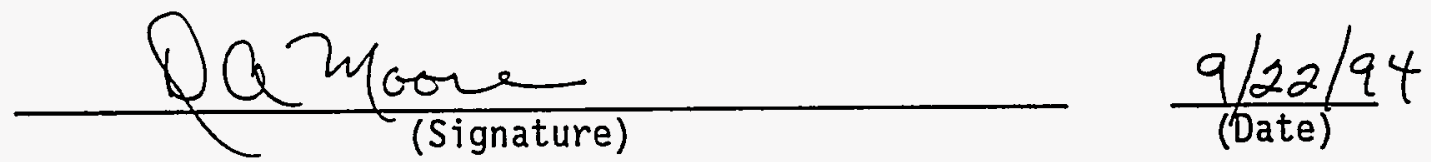

D. A. Moore (Acting)

(Printed Name)

* Approval for Activity Data Sheet 7332 - titled Pollution Prevention 


\author{
U.S.. DEPARTMENT OF ENERGY \\ RICHTAND OPERATIONS OFFICE \\ MANAGEMENT \& OPERATING (MEO). CONTRACT WORK AUTHORIZATION
}

2. Initiaror:

$\frac{R . G, \text { Folt }}{\text { Name and Signature }} \quad \frac{376-5340}{\text { Org. }}$ Telephone No.

3. Program Title: Environmental Support

4. Program WBS NO.: 1.5.2/7.4.11

5. Contractor: WRC

6. Performance Period From: 10/1/94 To: 9/30/95

7. Start Date:_N/A___Expected Completion Date: _N/A 8. Description: IIST ALI ADSS:

$\begin{array}{llllll}7330 & \text { Expenge } & \text { CENRTC } & \text { GPP } & \text { Iine Item } & \text { Total } \\ (\$ \mathrm{~K}) & \$ 8,162 & \$ 170 \mathrm{~K} & & & \$ 8,332 \\ 7360 & \text { Expense } & \text { CENRTC } & \text { GPP } & \text { Line Item } & \text { TotaI } \\ (\$ \mathrm{~K}) & \$ 2,701 & & & & \\ \mathbf{6 5 9 5} & \text { Expense } & \text { CENRTC } & \text { GPP } & \text { Iine Item } & \text { Total } \\ (\$ \mathrm{~K}) & \$ 369 & & & & \$ 369\end{array}$

This 15 an interim authorization to spend in accordance with the FY 1995 Environmental Support SrYPP. Expenditures should at no time exceed the funding shown above of in the FINPI.AN. A revised Work Authorization will be issued pending the FY 1995 budget reprioritination process in October 1994.

\title{
Concurrence
}

$$
\text { NAME . SIGNATURE DATE }
$$

9. RI Program Manager: R. G. Holt fR. Itolt $9 / 30 / 94$

10. Contractor: W. T. Dixon

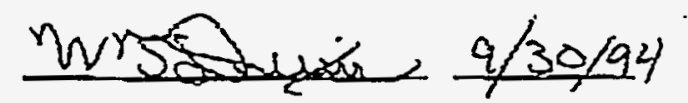

Agrioval

11. RI AM-COR: J. D. Bauer

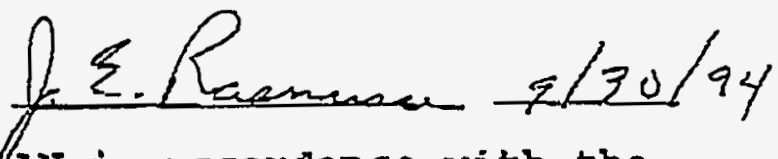

Funds will be authorized via the RINPLAN in accordance with the authoxized funding table.
C.E. OPI
CFO
MEO Contractor 


\section{RELEASE AUTHORIZATION}

Document Number: WHC-SP-1112

Document Title: $\quad$ Environmentai Support FY 1995 Multi-Year Program

Plan/Fiscal Year Work PIan WBS 1.5.2/7.4.11

Release Date: $\quad 9 / 28 / 94$

This document was reviewed following the procedures described in WHC-CM-3-4 and is:

APPROVED FOR PUBLIC RELEASE

***************

WHC Information Release Administration Specialist:

Christixi Mtillexighass

C. Willingham

$9 / 28 / 94$

(Signature)

(Date) 


\section{PREFACE}

The Multi-Year Program Plan (MYPP) is the programmatic planning baseline document for technical, schedule, and cost data. The MYPP contains data by which all work is managed, performed and controlled. The integrated planning process, defined by $R L$, is predicated on the establishment of detailed data in the MYPP.

The MYPP includes detailed information for the data elements including Level III critical path schedules, cost estimate detail, and updated technical data to be done annualiy. There will be baseline execution year and out year approval with work authorization for execution.

The MYPP will concentrate on definition of the scope, schedule, cost and program element level critical path schedules that show the relationship of planned activities.

The Fiscal Year Work Plan (FYWP) is prepared for each program to provide the basis for authorizing fiscal year work. This expansion of the programmatic details relates to schedules, resource estimates, costs, and work breakdown structure definitions.

Based on these data elements, the MYPP/FYWP will be structured into three main areas:

1. Program Overview (Program Vision and Mission, Program Mission Strategy)

2. Program Baselines (WBS Information, Responsibility Assignment Matrix, Program Logic Diagrams, Program Master Basel ine Schedule, Program Performance Baseline Schedule, Milestone List, Milestone Description Sheets, Program Cost Baseline, Basis of Estimate, Waste Type Data, Planned Staffing, Building Blocks)

3. Fiscal Year Work Plan (FYWP) (Cost Estimate Baseline by Month, Program - Funding Required, Carryover)

As the "cornerstone" program-planning baseline document, the MYPP is prepared and submitted in accordance with the RLID 5000.2, "Long-Range Planing Process."

Guidance found in RLID 5000.11, "Scheduling," is used for the logic and schedule formats. The MYPP/FYWP baseline is based on the budget and cost estimate data reflected in the Activity Data Sheets.

Validation of our baselines is now a requirement per RLP 5700.9, "Cost Estimating Validation." The scope, schedule, and cost data described in the MYPP/FYWP will meet this validation criteria. 
WHC-SP-1112

TABLE OF CONTENTS

1. Program Overview .................. . . . . . 1-1

1A. Program Vision/Mission ............... 1-2

1B. Program Mission Strategy .. . . . . . . . . . . 1-3

1B1. Technical Function and Requirements .... 1-4 - 1-10

1B2. Program Objectives ........... 1-11-1-12

1C. Planning Assumptions .............. . 1-13

1D. Issue and Constraints ............. . . 1-14

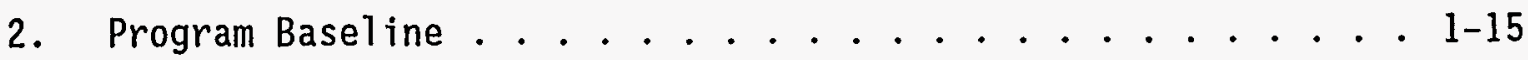

2A. WBS Informatiation ............... 1-16

2A1. WBS Structure ................ 1-17

2A2. WBS Dictionary ........... 1-18-1-19

2B. Responsibility Assignment Matrix . . . . . . . 1-20-1-21

2C. Program Logic Diagram . . . . . . . . . . . . 1-22

2D. Program Master Baseline Schedule (PMBS) . . . . 1-23 - 1-38

2E. Program Performance Base1 ine Schedule (PPBS) . . 1-39-1-83

2F. Milestone List ................ . 1-84-1-87

2G. Milestone Description Sheets ........ 1-88-1-135

2H. Cost Basel ine Summary by Year............ 1-136

2I. Basis of Estimate............... 1-137

2J. Waste Type Data .............. . 1-138 - 1-144

2K. Planned Staffing (Ful7-Time Equivalents) . . 1-145 - 1-146

2L. Building Blocks............. 1-147-1-156

3. Fiscal Year Work PTan (FYWP) . . . . . . . . . . . 1-157

3A. Cost Baseline Summary/Program Element - Operating Expense 1-158

3B. IProgram Summary/Cost Element - Operating Expense .. . 1-159

3C. Cost Baseline Summary - CENTRC . . . . . . . . . 1-160

3D. Program Funding .. . . . . . . . . . . . . . 1-161 
HANFORD ENVIRONMENTAL MANAGEMENT PROGRAM

FY 1995 MYPP

\title{
Section 1
}

\author{
Program Overview
}




\section{A. Program Vision/Mission}

The mission of the Hanford Environmental Management Program (HEMP) is to provide integrated guidance and support across mission areas to ensure the Hanford Site facilities/programs achieve compliance with environmental-requirements.

The following are specific assumptions made concerning environmental support:

* . Environmental Support will continue for all Hanford Site mission areas as long as the Site operates.

* As environmental compliance requirements change, these activities will be revised to ensure that compliance is maintained.

* The nature and extent of these activities will be modified as the Site cleanup mission progresses and decisions are made regarding cleanup strategy.

The HEMP role in sitewide activities is to act as a focal point for setting environmental policies and practices, and determining overall guidance; facility-specific compliance activities remain the responsibility of individual plants/programs. 
HANFORD ENVIRONMENTAL MANAGEMENT PROGRAM

\section{B. Program Mission Strategy}

The specific HEMP mission strategy is to provide guidance, coordination and integration for the following specific environmental activities:

* NEPA Coordination

* Environmental Field Services

* $\quad$ Air and Water Permitting Coordination

* Environmental Reporting

* Tri-Party Agreement Integration

* RCRA Compliance

* Pollution Prevention 


\section{B1. Technical Function and Requirements}

Provide sitewide support to management and facilities on overall NEPA/SEPA activities to ensure consistency/compliance. Primary activities include identification of NEPA/SEPA compliance requirements and participation in the development of strategies, identifying appropriate types of NEPA/SEPA documentation, preparing such documentation and supporting documents, response to and resolution of RL and DOE-HO document comments, and negotiations with RL and DOE-HO on NEPA compliance issues.

Regulatory Drivers: NEPA of 1969; 40 CFR 1500-1508; 10 CFR 1021; RL 5440.1A; DOE Order 5440.1E; 10 CFR 1022; WAC 197-11

Provide direct support to Hanford Site operations in attaining or maintaining compliance with environmental requirements. Provide coordination of onsite inspections by regulatory agencies, provide guidance and assistance in the preparation of regulatory compliance files, serve as a focal point for resolution of sitewide environmental compliance issues.

Regulatory Drivers: WAC 173-303; WAC 246-247; WAC 173-303-210 \& 380; WAC 173-303-646; WAC 173-303-070; 40 CFR 61; 40 CFR 246; DOE 5400.3; TriParty Agreement Article XXXVII, XXXVI 
HANFORD ENVIRONMENTAL MANAGEMENT PROGRAM

\section{B1. Technical Function and Requirements}

Participate in negotiations with federal, state, and local regulatory agencies in the establishment of air and water permitting priorities, schedules, and resolution of permitting issues, compliance plans and agreements involving Hanford Site Air and Water permitting activities. Establish and maintain standardized formats and procedures for all air and water permitting documentation. Respond to federal, state, and local agency requests for permitting documentation and associated information.

Regulatory Drivers:

WAC 173-400, 460, 401, 480; WAC 246-247; 40 CFR 52, 60, 61, 70; BCCAA Regulation 1; 40 CFR 122, 124; 33 CFR ACOE; WAC 173-218

Prepare the Emergency Planning and Community Right-to-Know Act (EPCRA) Sections 311, 312 , and 313 reports. The EPCRA reports are prepared for the entire Hanford Site and submitted to $\mathrm{RL}$ for transmittal to other agencies. Maintain the Hazardous Material Inventory Database (HMID) to collect and collate EPCRA reporting information for the Hanford Site. Update the Land Disposal Restrictions Report annually to meet the requirements of the TriParty Agreement and submit to $\mathrm{RL}$ to transmit EPA and Ecology. Prepare the Polychlorinated Biphenyl (PCB) Document Log and submit to RL. Prepare the PCB Annual Report and submit 
HANFORD ENVIRONMENTAL MANAGEMENT PROGRAM

\section{B1. Technical Function and Requirements}

to $\mathrm{RL}$ to transmit the report to the EPA. Prepare the Annual Dangerous Waste Report for the Hanford Site and submit to RL to transmit the report to the State and EPA. Prepare and complete the RCRA, Section 3016 Biennial Report and submit to RL to transmit to the State and EPA. Report spills and releases of hazardous substances to the environment in compliance with federal and state regulations.

Regulatory Drivers:

40 CFR 355, 370, 372; 29 CFR 1910.1200; WAC 173-303-220, 390, 140; 40 CFR 761; 40 CFR 264.75, 40 CFR 265.56; 40 CFR 268; 40 CFR 260; RCRA, Section 3016; Clean Air Act Amendments of 1990; Federal Facility Compliance Act of 1992; 40 CFR 302, 355; DOE 5484.1; Gen. Reg. 80-7, Tri-Party Agreement; WAC 173-303-145; RL 5484.1, DOE 5000.3B; National Pollutant Discharge Elimination Permit System Permit WA 000374-3, City of Richland Ordinance 35-84

Provide administration of the Tri-Party Agreement. The Tri-Party Agreement sets the plan and schedule for achieving regulatory compliance and cleanup of waste sites at the Hanford Site. Specific administration requirements (change control, progress reporting, milestone tracking, Tri-Party Agreement amendments, data management dispute resolution, etc.) are legal requirements of the Tri-Party Agreement. The Tri-Party Agreement will be formally updated annually. Meetings will be held with EPA and Ecology to discuss progress. 
HANFORD ENVIRONMENTAL MANAGEMENT PROGRAM

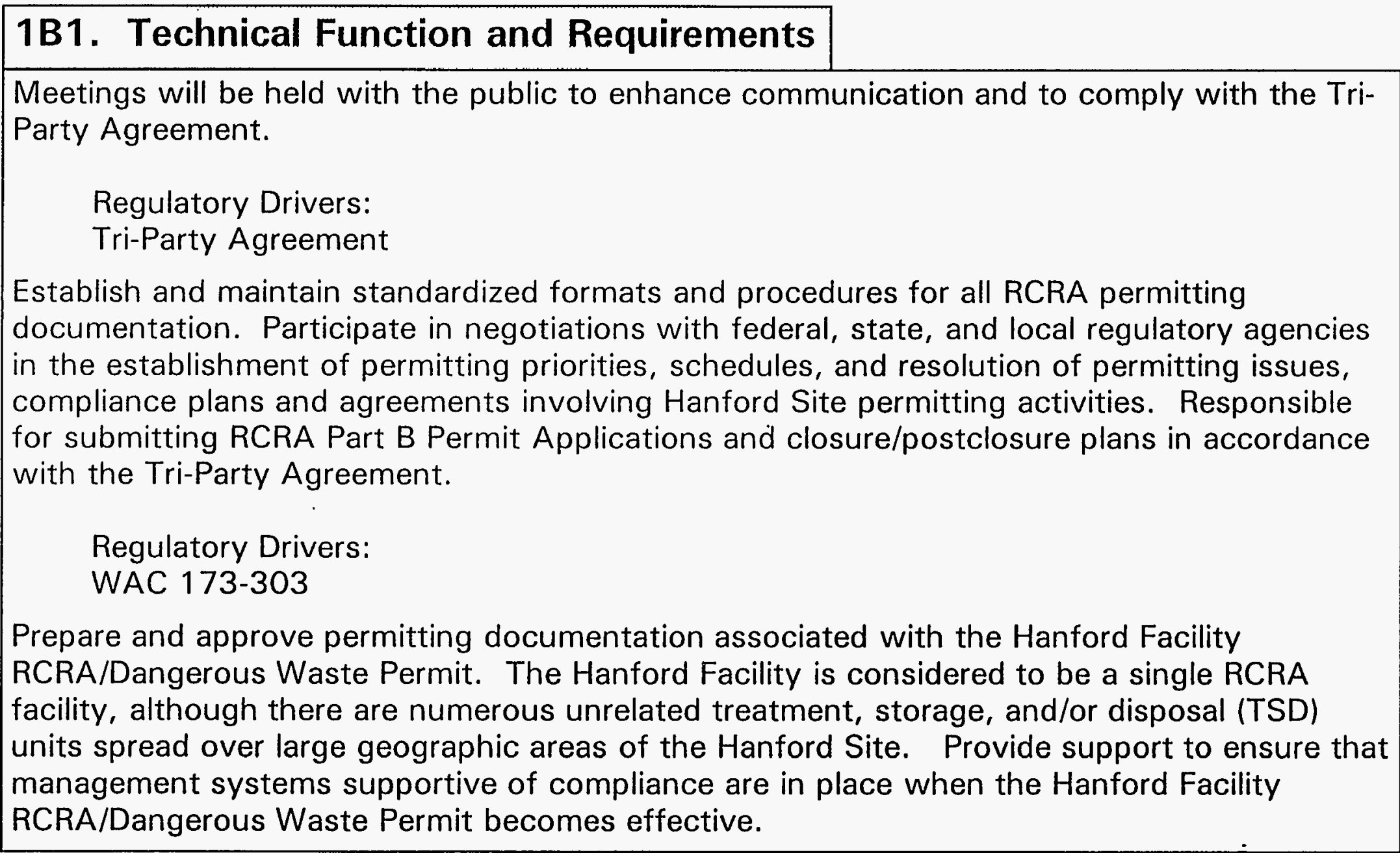




\section{B1. Technical Function and Requirements}

A coordinated document control system is required to ensure that (1) all affected TSD units and TSD activities are working from the most current RCRA Permit documents and attachments and (2) changes to these documents are distributed, incorporated, and implemented in a timely manner.

Additional TSD units will be added periodically to the RCRA Permit through the permit modification process. In addition, changes made to TSD units already incorporated into the RCRA Permit will be subject to the permit modification requirements. Therefore, a system will be in place that will be used to modify the RCRA Permit.

Due to the complexity of the RCRA Permit, a condition tracking system will be developed/maintained that lists all of the General Facility and Unit-Specific conditions that must be met.

Develop and maintain the recordkeeping and reporting system (1) to ensure that the specified records are being maintained in the Hanford Facility Operating Record and (2) to identify which records, within the Hanford Facility Operating Record, will be kept at the Hanford Facility level and which records will be kept at the TSD unit level. Provide annual reports covering (1) projections of anticipated costs for closure and postclosure of TSD units incorporated into the RCRA Permit and (2) waste minimization. 


\section{B1. Technical Function and Requirements}

Develop and maintain Solid Waste Management Units (SWMU) Inventory. Include the location, type and current status of each SWMU, date(s) the SWMU was operated, general dimensions and description of each SWMU, and general descriptions of waste placed in the SWMU.

Provide general facility inspection support of the Hanford Facility, excluding TSD units already covered by unit-specific plans. The purpose of the general inspection is to prevent malfunctions and deteriorations, operator errors, and discharges that might cause or lead to the release of dangerous waste constituents to the environment or pose a threat to human health.

Provide support to the mapping and marking of dangerous waste underground pipelines.

Regulatory Drivers:

Hanford Facility RCRA/Dangerous Waste Permit; WAC 173-303

Develop a sitewide pollution prevention (P2) program. Provide pollution prevention support for the Hanford Site to ensure that federal, state, and DOE regulations, policies, and reporting requirements are met. Complete and submit the Pollution Prevention Plan progress report, DOE Annual Waste Generation and Waste Minimization report, EPA Biennial Report 


\section{B1. Technical Function and Requirements}

on Waste Minimization, and the Dangerous Waste Pilot Report. It is the responsibility of the specific facilities/programs to develop and implement P2, including incorporation into facility design/modification.

Regulatory Drivers:

DOE Order 5400.1; DOE Order 5820.2A; WAC 173-303; WAC 173-307; RCRA Section 3002 (a)(6); RCRA Section 3005(h); Executive Order 12843, 12856, 12873, 12902 


\section{B2. Program Objectives}

The HEMP was established in 1986 to incorporate a structured approach toward achieving environmental compliance at the Hanford Site. The program scope focuses on regulatory compliance support by identifying and resolving sitewide environmental issues, providing guidance to facilities on compliance activities, coordinating sitewide report preparation, and providing integration of Tri-Party Agreement activities.

Key program objectives place high priority on environmental compliance. These objectives are:

* Ensure the safety of operations.

* Ensure program priorities are consistent with environmental regulations.

* Ensure that compliance with environmental requirements are identified in Facility/Program budgets.

* Ensure submittal of mandatory State and Federal regulatory reports. 
HANFORD ENVIRONIMENTAL MANAGEMENT PROGRAM

\section{B2. Program Objectives}

(Continued):

* Provide key contact within WHC for resolution of sitewide environmental compliance issues.

* Ensure appropriate resources are identified to support coordination and integration of TriParty Agreement requirements for the Hanford Site mission.

* Provide integration of NEPA, RCRA, and Air and Water permitting activities to ensure consistency and compliance.

* Comply with State and Federal regulations, and DOE requirements for waste minimization, waste reduction and pollution prevention. 
HANFORD ENVIRONIMENTAL MANAGEMENT PROGRAM

\section{C. Planning Assumptions}

The key assumptions in HEMP planning are:

* The FY 1995 MYPP reflects the total cost of work scope agreed to in the Integrated

Target Case. This baseline assumes a productivity commitment $110 \%$ in FY $1995,10 \%$ in FY 1996, 15\% in FY 1997, 20\% in FY 1998, 10\% in FY 1999, 10\% in FY 2000 and $10 \%$ in FY 2001) which achieves the same work scope at a lower unite rate, or the application of more efficient processes, or through cost avoidance.

* The FY 1995 MYPP assumes approval of the FY 1994 Waste Management/Facility Transition reprogramming. For the HEMP this total is $\$ 0.5 \mathrm{M}$.

* More compliance items are expected as new regulations and compliance orders are implemented.

* Facility specific compliance activities remain the responsibility of individual plants/programs.

* The Hanford Facility RCRA/Dangerous Waste Permit is expected to be issued in FY 1994, creating expanded regulatory requirements. TSD unit specific requirements of the Permit will be the responsibility of individual facilities/programs.

* The P2 Program has been elevated to a separate program element as the first step toward becoming its own independent program. 
HANFORD ENVIRONMENTAL MANAGEMENT PROGRAM

\section{D. Issuies and Constraints}

The Issues and Constraints for the HEMP are:

* The Hanford Facility RCRA/Dangerous Waste Permit is expected to be issued in FY 1994, creating additional compliance requirements. When fully defined these requirements may result in new funding impacts for FY 1995 and out years.

* The HEMP is assuming approval of the FY 1994 Waste Management/Facility Transition reprogramming. For HEMP this total is $\$ 0.5 \mathrm{M}, \$ 0.3 \mathrm{M}$ is required to perform the deferred FY 1994 anticipated carryover work scope.

* $\quad$ Beginning in FY 1994 the Hanford Site DOE and Executive Order requirements for P2 increased substantially, thereby requiring additional funding in FY 1995 to continue supporting the additional scope. 


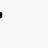



WBS 1.5.2.1

Section 2

Program Baseline 
$9 L-L$

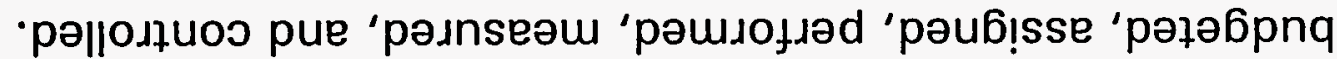
'pә|nрәчәs 'pәuuejd 'pәu!

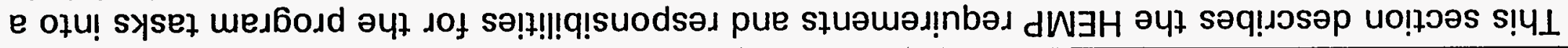
uolieuinojui $\operatorname{sgM}: \forall Z$ 
HANFORD ENVIRONMENTAL MANAGEMENT PROGRAM

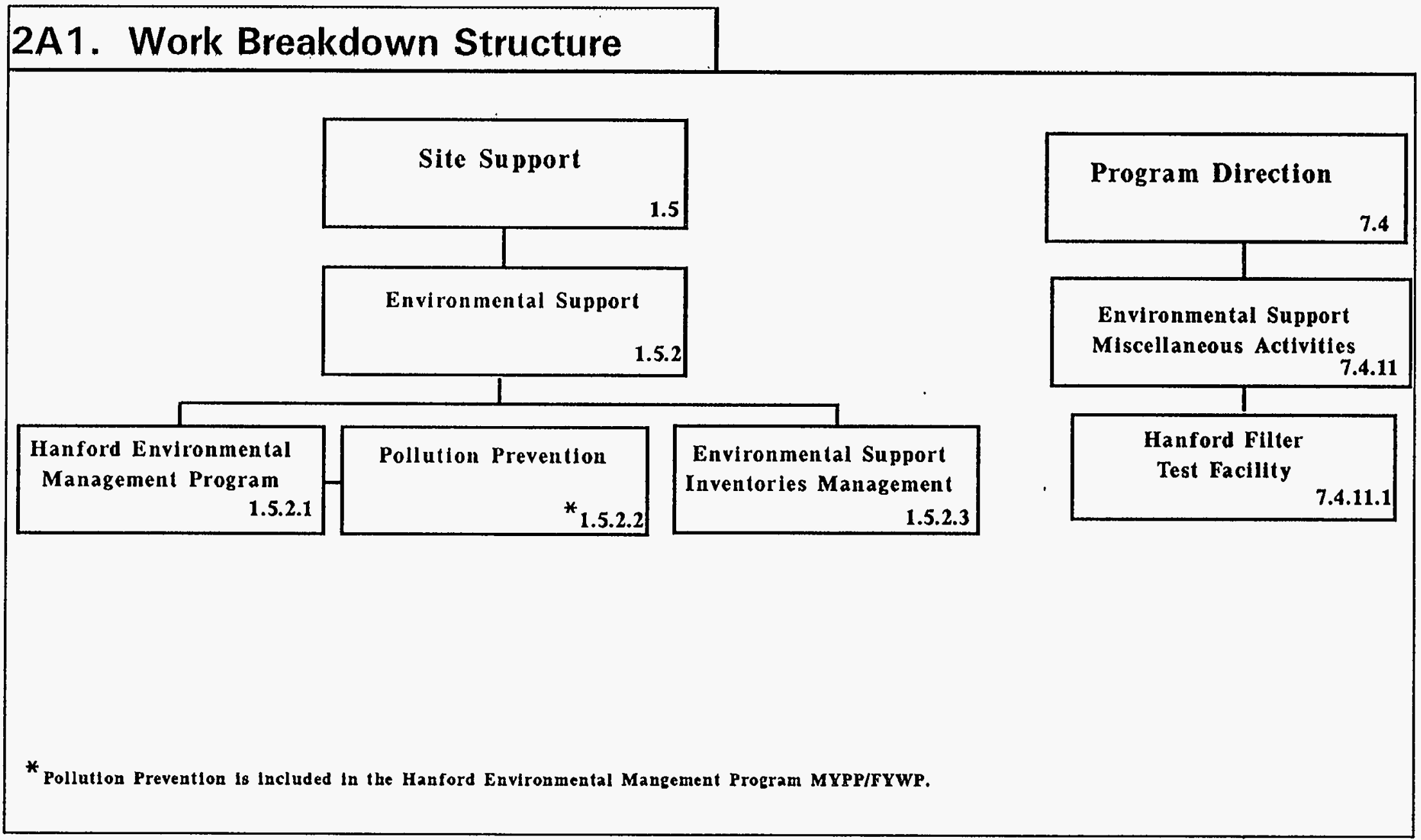




\section{A2. WBS Dictionary}

WORK BREAKDOWN STRUCTURE

\begin{tabular}{|c|c|c|}
\hline $\begin{array}{l}\text { 1. PROJECT TITLE/PARTICIPANT } \\
\text { Henford Environmental Mansgement Program }\end{array}$ & 2. DATE & $\begin{array}{l}\text { 3. DOE-HQ WBS CODE: } \\
3.7 .6 .5\end{array}$ \\
\hline $\begin{array}{l}\text { 4. DOE-RL WBS ELEMENT CODE } \\
\text { H. } 15,2,1 \% \text { का }\end{array}$ & \multicolumn{2}{|c|}{$\begin{array}{l}\text { 5. WBS ELEMENT TITLE } \\
\qquad \text { Hanford Environmental Management Program }\end{array}$} \\
\hline $\begin{array}{l}\text { 6. CONTRACTOR WBS ELEMENT CODE: } \\
31 \mathrm{RHH} \\
\end{array}$ & $\begin{array}{l}\text { 7. REVISION \# AND AUTHORIZATION } \\
\text { Not Applicable }\end{array}$ & $\begin{array}{l}\text { 8. DATE } \\
\text { Not Applicable }\end{array}$ \\
\hline 9. APPROVED CHANGES & $\cdot$ & \\
\hline $\begin{array}{l}\text { 10. SYSTEM DESIGN DESCRIPTION } \\
\text { NUMBER: } \\
\text { TITLE: }\end{array}$ & $\begin{array}{l}\text { 11. BUDGET AND REPORTING NUMBER } \\
\text { EW-31-10-01-0 }\end{array}$ & \\
\hline
\end{tabular}

12. ELEMENT TASK DESCRIPTION

A. Cost Content

Refer to Section II part $2 \mathrm{H}$ for the Cost Baseline Summary.

B. Technica! Content/Work Statement

This program is divided into seven subactivities which are titled as follows: Program Administration, NEPA Coordination, Environmental Field Services, Air and Water Permitting, Environmental Reporting. Tri-Party Agreement Integration. RCRA Compliance.

Program Administration provides a focus of responsibility and authority for managing the HEMP. Provides overall coordination, direction and customer interface. Program priorities are established and administrative support is provided for program documentation, funds management, scheduling. and to perform Site Management System requirements.

NEPA Coordination provides sitewide support to management and facilities on overall NEPA/SEPA activities to ensure consistency/compliance.

Environmental Field Services provides coordination for onsite inspections by regulatory agencies, guidance and assistance in the preparation of regulatory compliance files at Treatment, Storage and Disposal facilities, focal point for resolution of sitewide specific environmental compliance issues.

Air and Water Permitting provides sitewide non-RCRA environmental permitting coordination and review/resolution of permitting issues, and review non-RCRA permitting activities at WHC for consistency.

Environmental Reporting includes: Preparation of mandatory regulatory reports (i.e. EPCRA 311 - MSDS Quarterly Update Lists, EPCRA 312 Hazardous Chemical Inventory Report, EPCRA 313 - Toxic Release Inventory Report. Hanford Site Annual Dangerous Waste Report, Hanford Sito Annual PCB Report, Hanford Site Annual PCB Log. Biennial RCRA 3016 Report), and Land Disposal Restriction Status (LDR) reporting (Tri-Party Agreement $\mathrm{M}-26$ ) and sitewide spill reporting guidance..

Tri-Party Agreement Integration provides for administration of Tri-Party Agreement activities across the mission aress. Specific administration requirements (change control, progress reporting. milestone tracking. Tri-Party Agreement amendments, data management dispute resolution, etc.) are legal requirements of the Tri-Party Agreement. Required reports will be prepared and participant/public meatings will be coordinated.

RCRA Complianco will prepare and approve permitting documentation associated with the Hanford Facility RCRA/Dangerous Waste Pormit. Support is provided to ensure that management systems supportive of compliance are in place when the Hanford Facility RCRA/Dangerous Wasto Permit becomes effective. In addition RCRA permirting also supports preparation of permit applications and closure/postclosure plans for sitewide environmental permits to address RCRA requirements (Tri-Party Agreement milestono M-20-00). This does not include unitspecific RCRA permit requirements and coordination. 


\section{A2. WBS Dictionary}

WORK BREAKDOWN STRUCTURE

\begin{tabular}{|c|c|c|}
\hline $\begin{array}{c}\text { 1. PROJECT TITLE/PARTICIPANT } \\
\text { Pollution Provention }\end{array}$ & $\begin{array}{l}\text { 2. DATE } \\
\text { September 21. } 1994\end{array}$ & $\begin{array}{c}\text { 3. DOE-HQ WBS CODE: } \\
3.7 .1 .3\end{array}$ \\
\hline $\begin{array}{c}\text { 4. DOE-RL WBS ELEMENT CODE } \\
1.5 .2: 2\end{array}$ & $\begin{array}{l}\text { 5. WBS ELEMENT TITLE } \\
\text { Pollution Prevention }\end{array}$ \\
\hline $\begin{array}{c}\text { 6. CONTRACTOR WBS ELEMENT CODE: } \\
\text { 1R1H8 }\end{array}$ & $\begin{array}{c}\text { 7. REVISION \# AND AUTHORIZATION } \\
\text { Not Applicable }\end{array}$ & $\begin{array}{c}\text { 8. DATE } \\
\text { Not Applicablo }\end{array}$ \\
\hline
\end{tabular}

9. APPROVED CHANGES

10. SYSTEM DESIGN DESCRIPTION NUMBER: TITLE:
11. BUDGET AND REPORTING NUMBER

EW-31-10-01-0

12. ELEMENT TASK DESCRIPTION
A. Cost Content

Refer to Section II part $2 \mathrm{H}$ for the Cost Baseline Summary.

B. Technical Content/Work Statement

Develop a sitewide pollution prevention (P2) program. Provide pollution prevention support for the Hanford Site to ensure that federal, state, and DOE regulations, policies, and reporting requirements are met. Complete and submit the Pollution Prevention Plan progress report, DOE Annual Waste Generation and Waste Minimization report, EPA Biennial Report on Waste Minimization, and the Dangerous Waste Pilot Report. It is the responsibility of the specific facilities/programs to develop and implement P2, including incorporation into facility design/modification.

RL hes designated WHC as the Hanford Site P2 lead and RL point of coordination for all other site contractors. WHC will coordinate P2 onsite according to the key elements for the sitewide and generator-specific programs identified in the 1994 DOE Waste

Minimization/Pollution Prevention (WMin/PP) Crosscut Plan. 


\begin{tabular}{|c|c|c|c|c|c|}
\hline 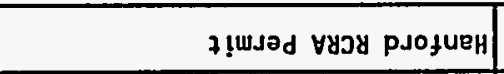 & כyunงg כy & bu!xjsew pue 6u!ddew au! jəd!d & $-7 \cdot 2 \cdot 1 \cdot Z^{\prime} \cdot S^{\circ} !$ & & \\
\hline 7!wsәd уу рлоңчен & əxundg כy & 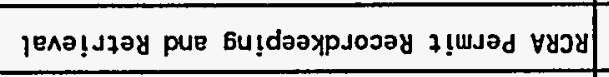 & $-\varepsilon^{*} L^{\prime} l^{\varepsilon \cdot Z^{\circ} \cdot S^{*} l}$ & & \\
\hline 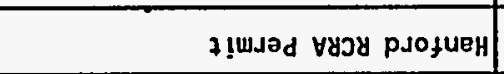 & әxunsg כy & 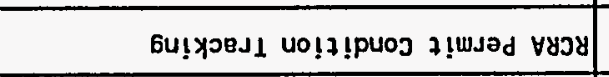 & $-2 \cdot L \cdot I^{2 L z^{\prime} \cdot S^{*}}$ & & \\
\hline 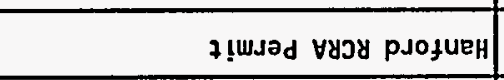 & əxundg כy & 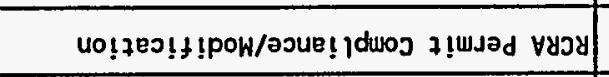 & $-1 \cdot \angle \cdot 1 \cdot 2 \cdot 5 \cdot 5 \cdot 1$ & & \\
\hline$\forall / N$ & $\forall / N$ & 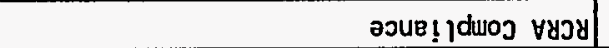 & & $L \cdot l \cdot 2 \cdot s \cdot l$ & \\
\hline 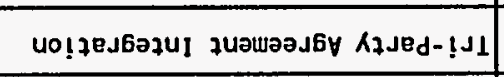 & plousy oา & 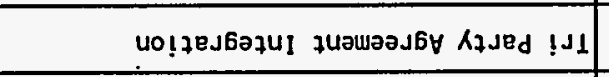 & $-1 \cdot 9 \cdot 1 \cdot 2 \cdot 5 \cdot 1$ & & \\
\hline$\forall / N$ & $\forall / N$ & 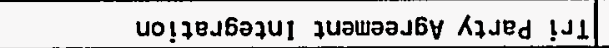 & & $9 \cdot 1 \cdot 2 \cdot 5 \cdot 1$ & \\
\hline szjodəy jequeursos!nug & pndjoys or & Gu!zjoday snzezs ә7sem y07 & $\begin{array}{r}\varepsilon 5 H L V \mid \\
-\varepsilon \cdot s^{2} l \cdot \tau^{*} s^{*} l \\
\end{array}$ & & \\
\hline s7jodey jequeuruos!nug & pnsjoxs or & 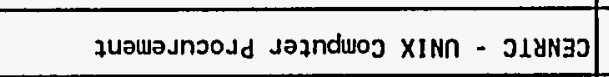 & $\begin{array}{r}25 H ! 4 z \\
-z \cdot s \cdot 1 \cdot z \cdot s \cdot l\end{array}$ & & \\
\hline słsoday jequaurostaug & pndjoxs or & S7soday jezuauros!nua & 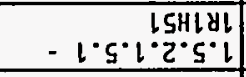 & & \\
\hline$\forall / N$ & $\forall / N$ & 6u! 7soday jequauruos!nua & & $s \cdot l \cdot 2 \cdot 5 \cdot l$ & \\
\hline 6u!ł7!Wدәd JajeM pue $J ! \forall$ & כxח & 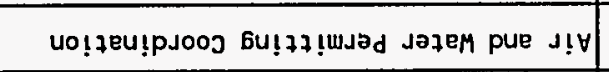 & $\begin{aligned} 1 \cdot 7 \cdot 1 \cdot 2 \cdot 5 \cdot 1 \\
\end{aligned}$ & & \\
\hline$\forall / N$ & $\forall / N$ & 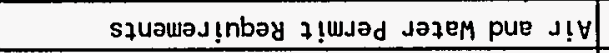 & & $\eta \cdot 1 \cdot 2 \cdot 5 \cdot 1$ & \\
\hline sos!̣jas Pןo!f gequauruoj!nug & uospue) $1 \exists 98$ & 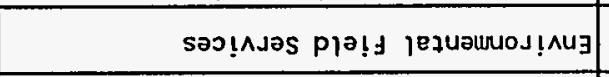 & 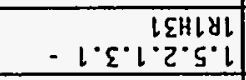 & & \\
\hline$\forall / N$ & $\forall / N$ & sas!njas pja!f jequamuoj!nug & & $\varepsilon \cdot l \cdot z \cdot s \cdot l$ & \\
\hline saj!̣ıJas $\forall d \exists N$ & чиешןәБиз ну & Uo!jeu!pJooJ $\forall d \exists N$ & 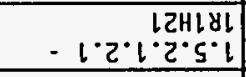 & & \\
\hline$\forall / N$ & $\forall / N$ & Uo!jeu!pJooj $\forall d \equiv N$ & & $2 \cdot 1 \cdot 2 \cdot 5 \cdot 1$ & \\
\hline jOJzUOJ mej60.d & sJamod 77 & 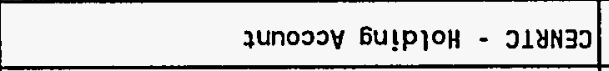 & 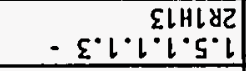 & & \\
\hline josquOJ wes6odd & sjamod & 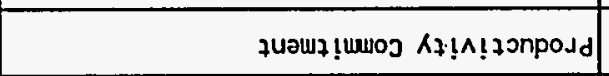 & $\begin{array}{r}2 ! H ! \cdot ! \\
-2 \cdot 1 \cdot 1 \cdot 2 \cdot 5 \cdot 1\end{array}$ & & \\
\hline jojquoj wej60Jd & S.JMOd 77 & uo!jejzs!u!wpy wej6odd & $-1 \cdot 1 \cdot 1 \cdot 2 \cdot 5 \cdot \frac{1}{L}$ & & \\
\hline$\forall / N$ & $\forall / N$ & Uo!̣es7s!u! upy weJ6osd & & $l \cdot l \cdot 2 \cdot s \cdot l$ & \\
\hline 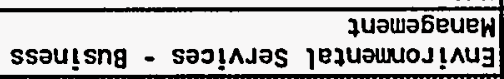 & (6U!2OY) כJ00W $\forall 0$ & wes6osd zuamabeueh jezuaunod!nug psofueh & & & $1 \cdot 2 \cdot 5 \cdot l$ \\
\hline NoI1YZINY9YO כา8ISNOdS3Y & УЭכษNษK $378 I S N O d S \exists Y$ & 37111 & INกOJJY ISOJ & MLIAIIJY & INJW \\
\hline
\end{tabular}




\section{B1. Responsibility Assignment Matrix}

\begin{tabular}{|c|c|c|c|c|c|}
\hline PROGRAM ELEMENT & ACTIVITY & COST ACCOUNT & l litLE & RESPONSI BLE MANAGER & RESPONSIBLE ORGANIZATION \\
\hline & & $\begin{array}{l}1.5 .2 .1 .7 .5- \\
\text { 1R1H75 }\end{array}$ & $\begin{array}{l}\text { Annual Closure Cost Estimate and Waste } \\
\text { Minimization Reports }\end{array}$ & RC Brunke & Hanford RCRA Permit \\
\hline & & $\begin{array}{l}1.5 .2 .1 .7 .6- \\
\text { IRIH76 }\end{array}$ & RCRA Permits Waste Inventory & RC Brunke & Hanford RCRA Permit \\
\hline & & $\begin{array}{l}1.5 .2 .1 .7 .7- \\
1 \mathrm{R} 1 \mathrm{H} 77\end{array}$ & Facility-Wide Inspection & BG Erlandson & Environmental Field Services \\
\hline & & $\begin{array}{l}1.5 .2 .1 .7 .8- \\
\text { IR1H78 }\end{array}$ & RCRA Permitting Coordination & SM Price & RCRA Permitting \\
\hline & & $\begin{array}{l}1.5 .2 .1 .7 .9- \\
2 R 1 H 79\end{array}$ & CENRTC - Records Keeping Computer System & RC Brunke & Hanford RCRA Permit \\
\hline 1.5 .2 .2 & & & Pollution Prevention & $N / A$ & $\mathrm{~N} / \mathrm{A}$ \\
\hline & 1.5.2.2.1 & & Pollution Prevention & DS Merry & Reengineering Program office \\
\hline & & $\begin{array}{l}1.5 .2 .2 .1 .1- \\
\text { IR1HB1 }\end{array}$ & Pollution Prevention & DS Merry & Reengineering Program office \\
\hline
\end{tabular}




\section{C. Program Logíc Diagram}

This page intentionally left black. 


\section{D. Program Master Baseline Schedule (PMBS)}

See attached. 


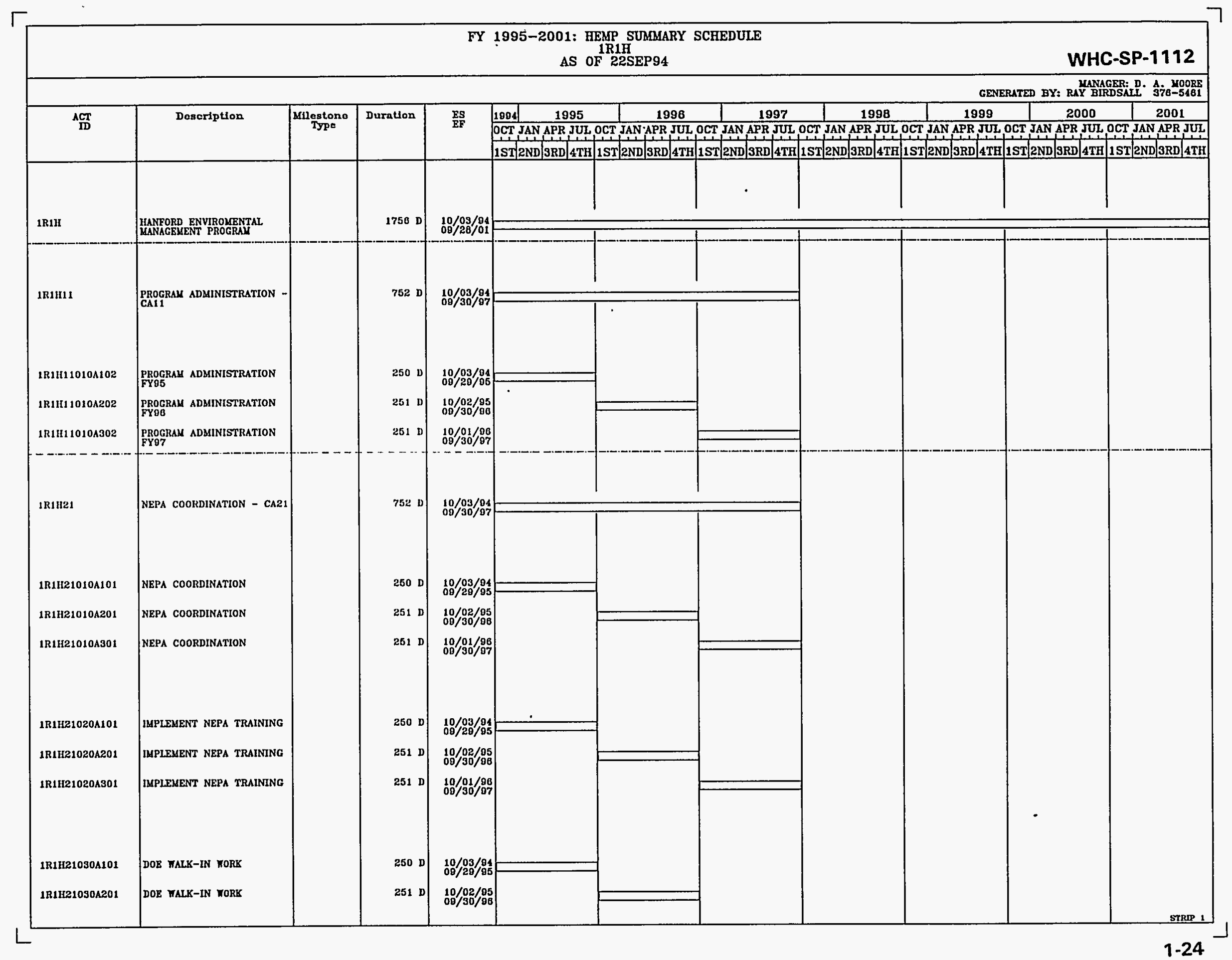




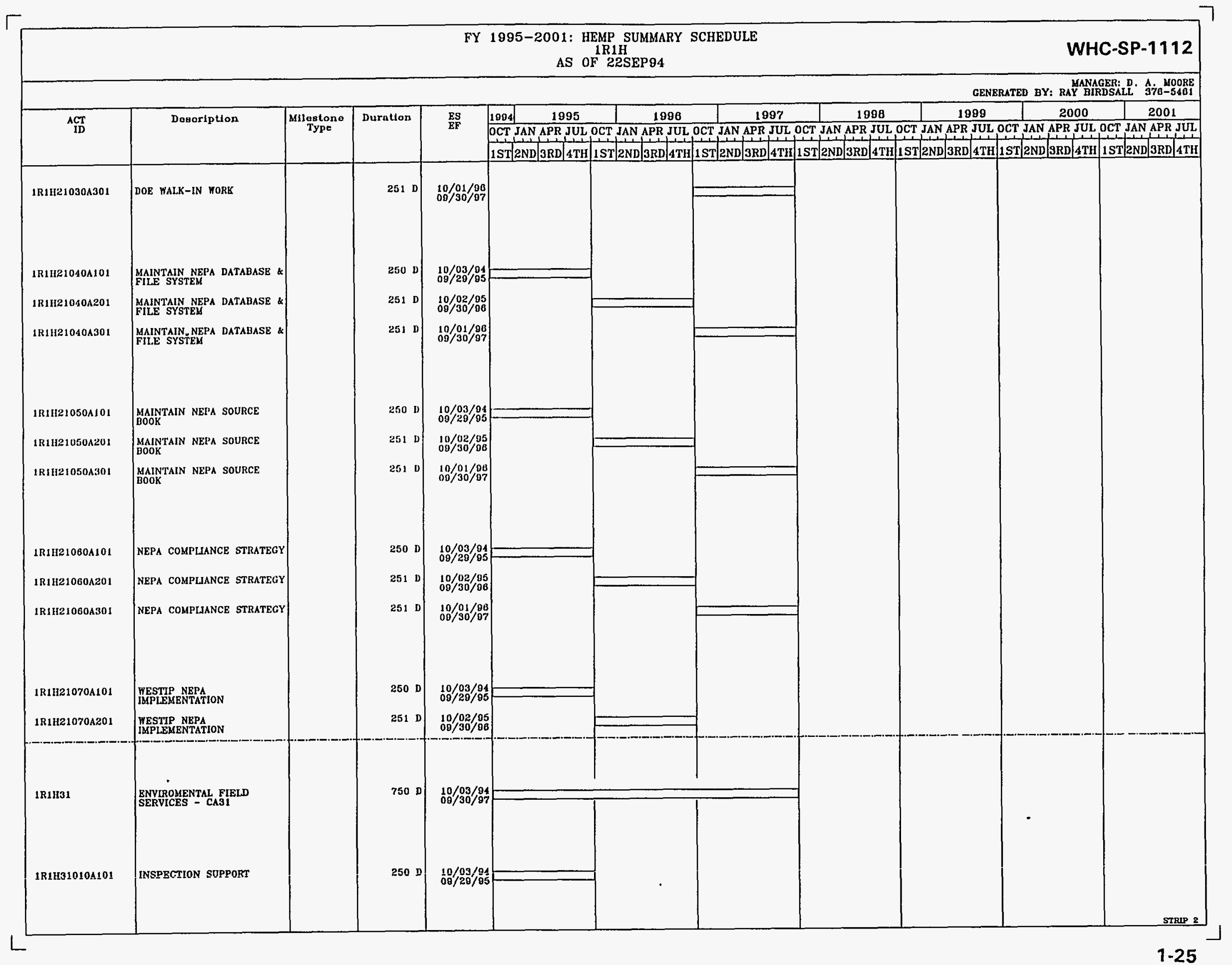




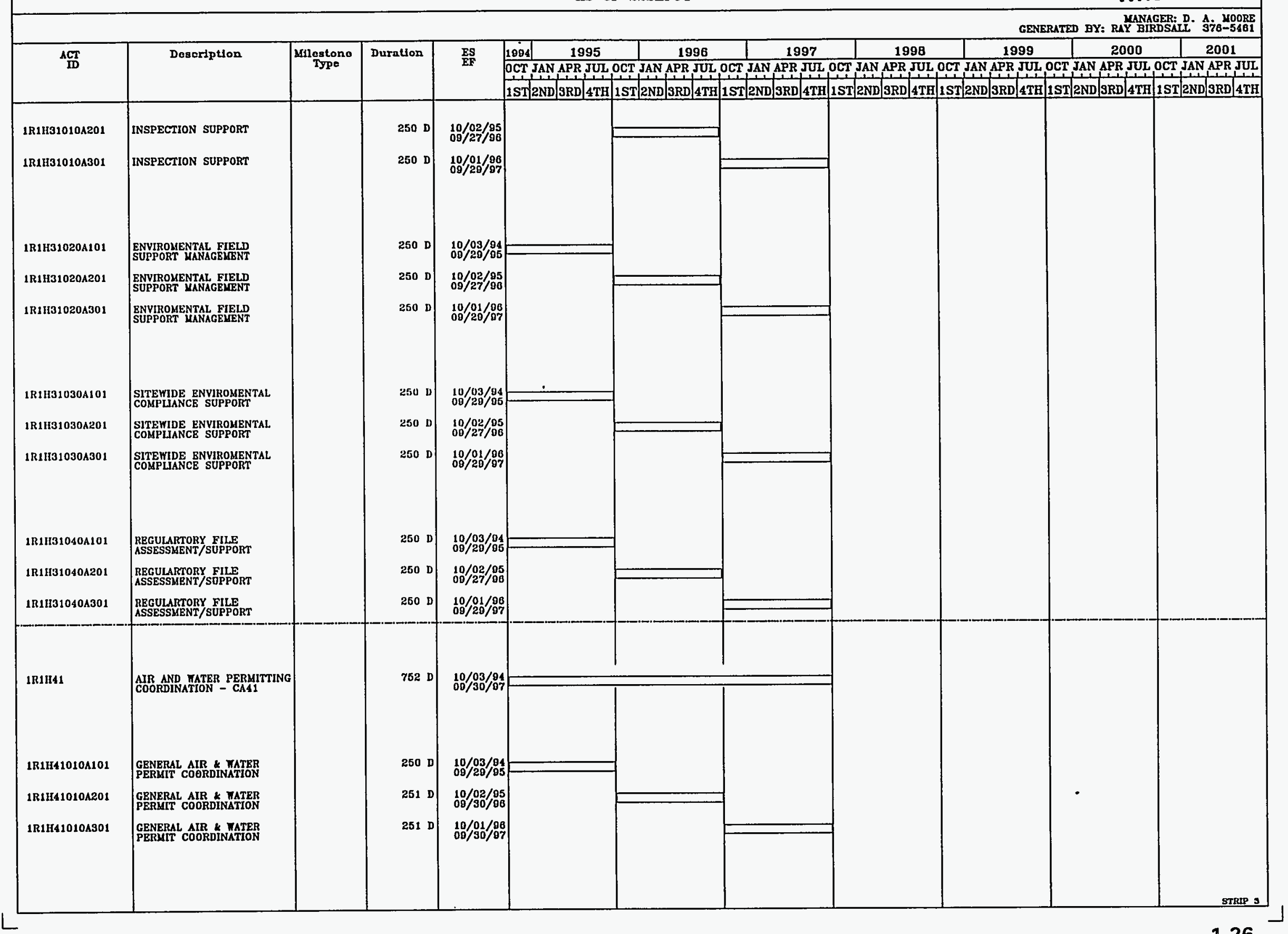




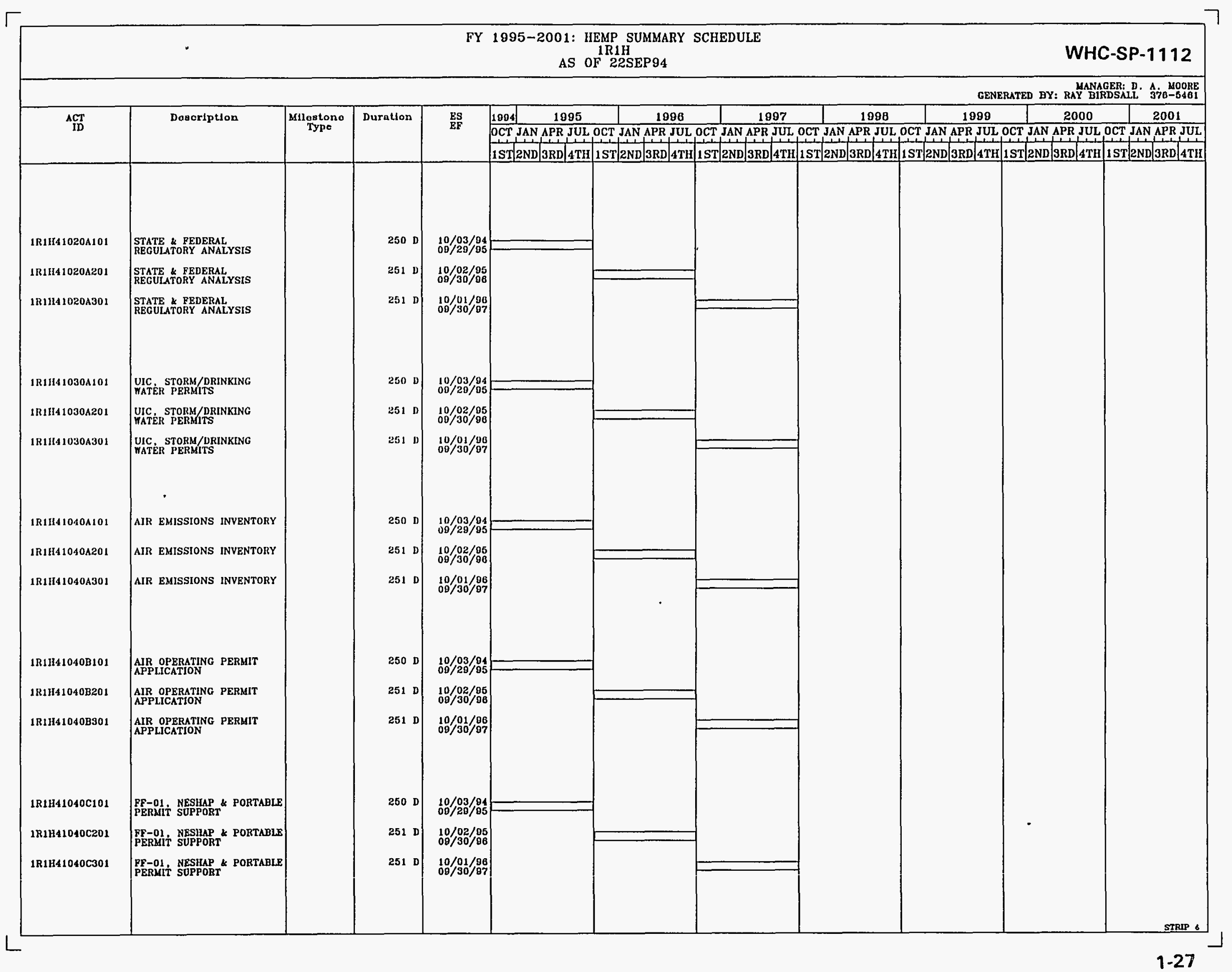




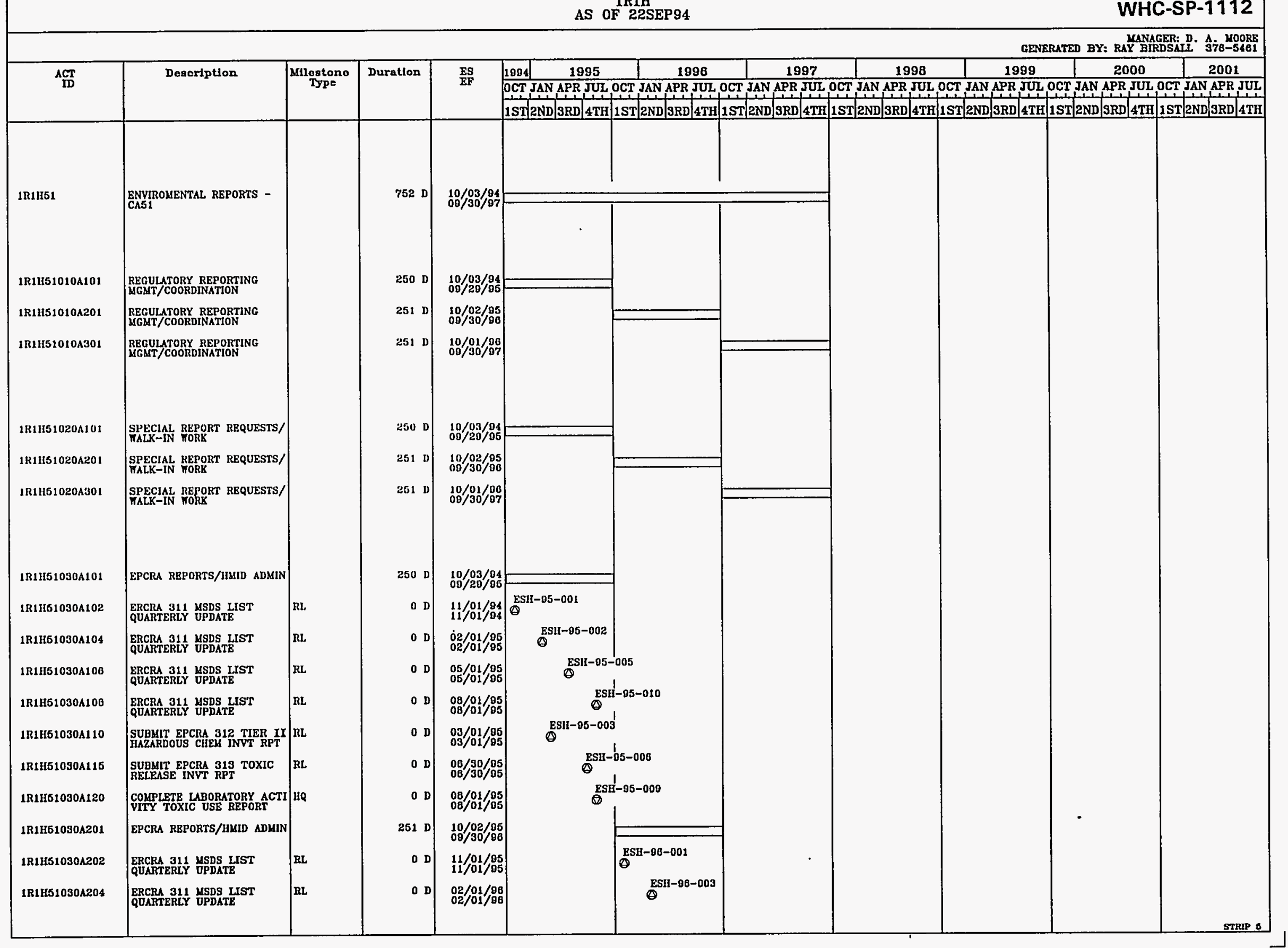




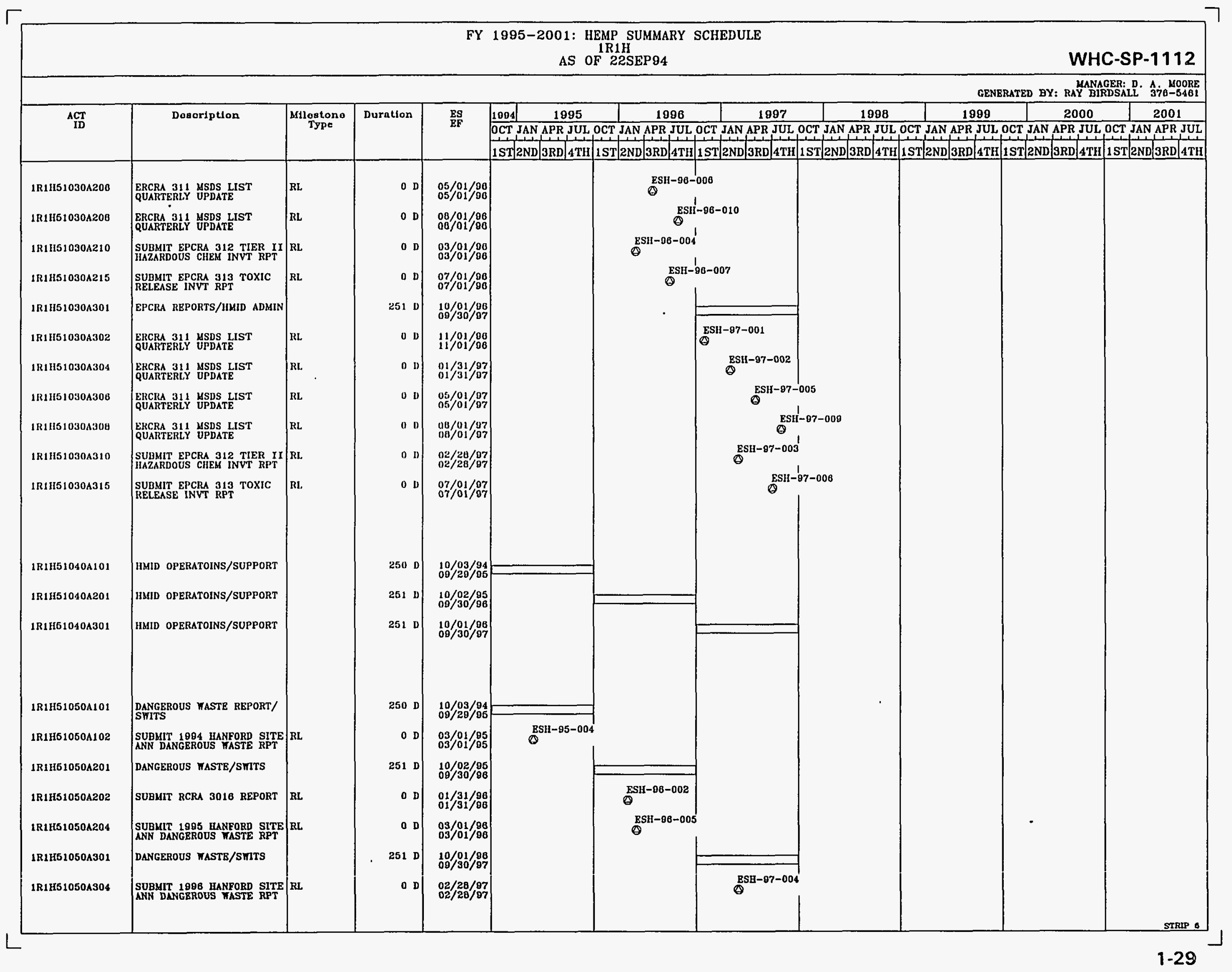




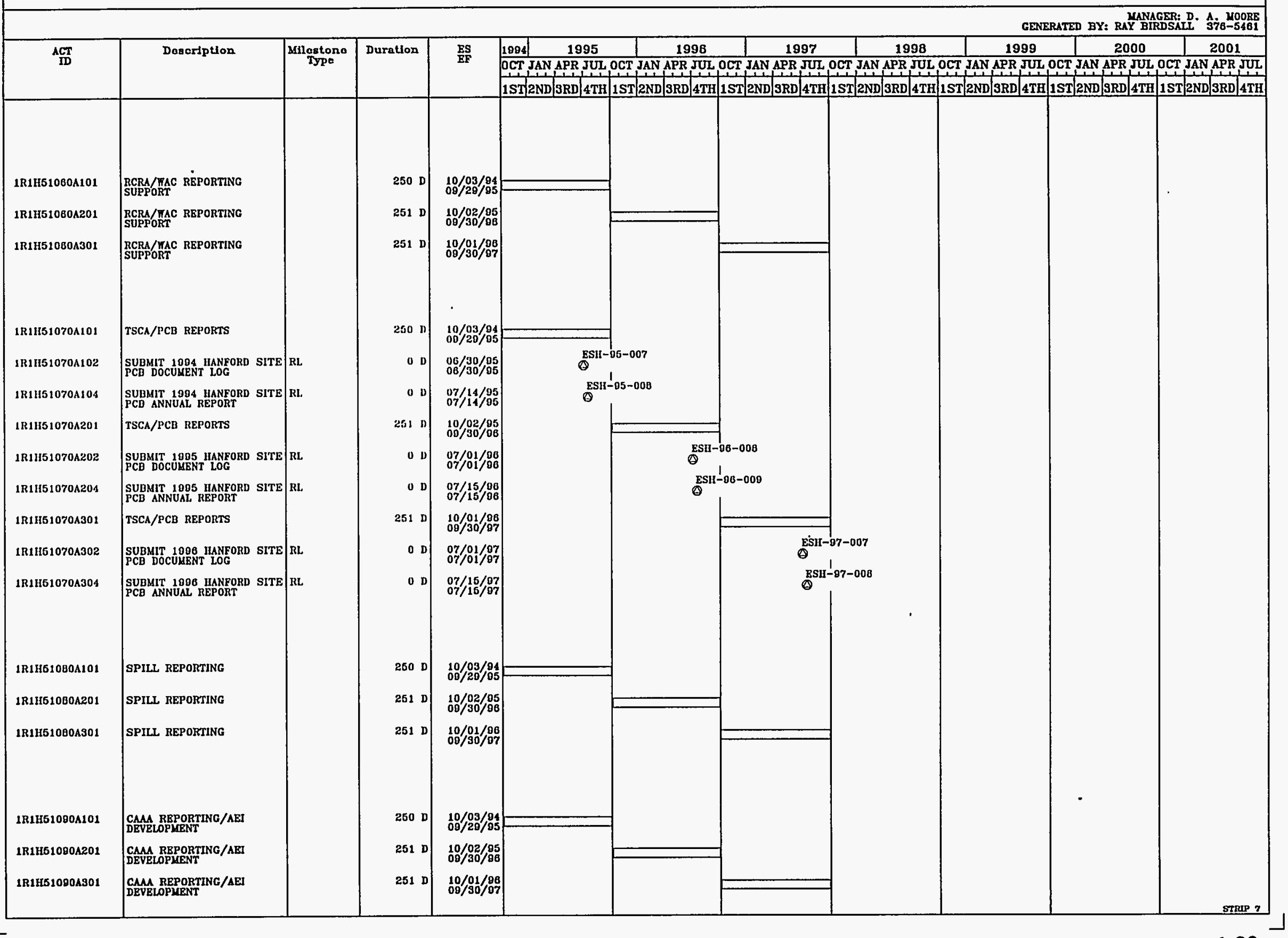




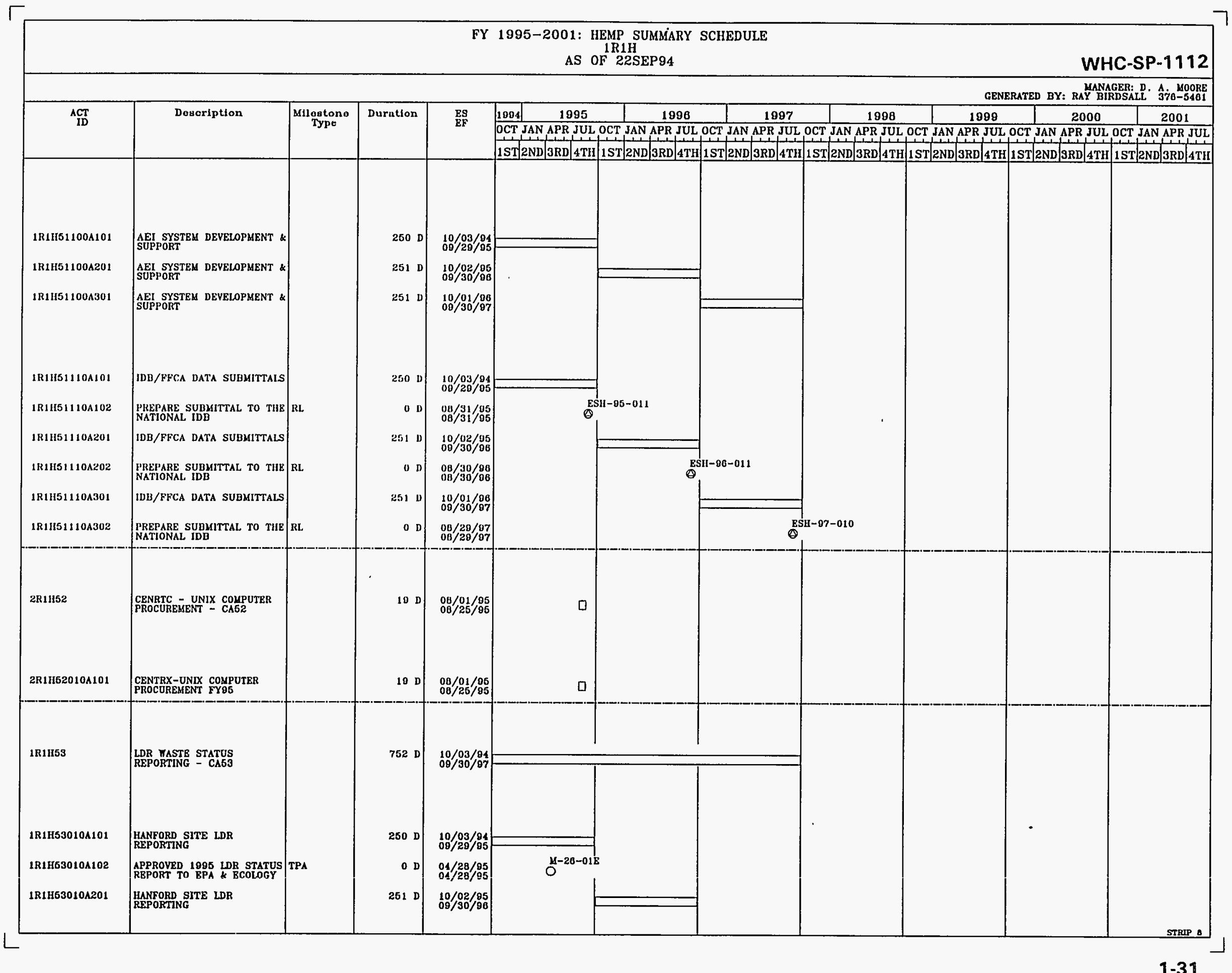




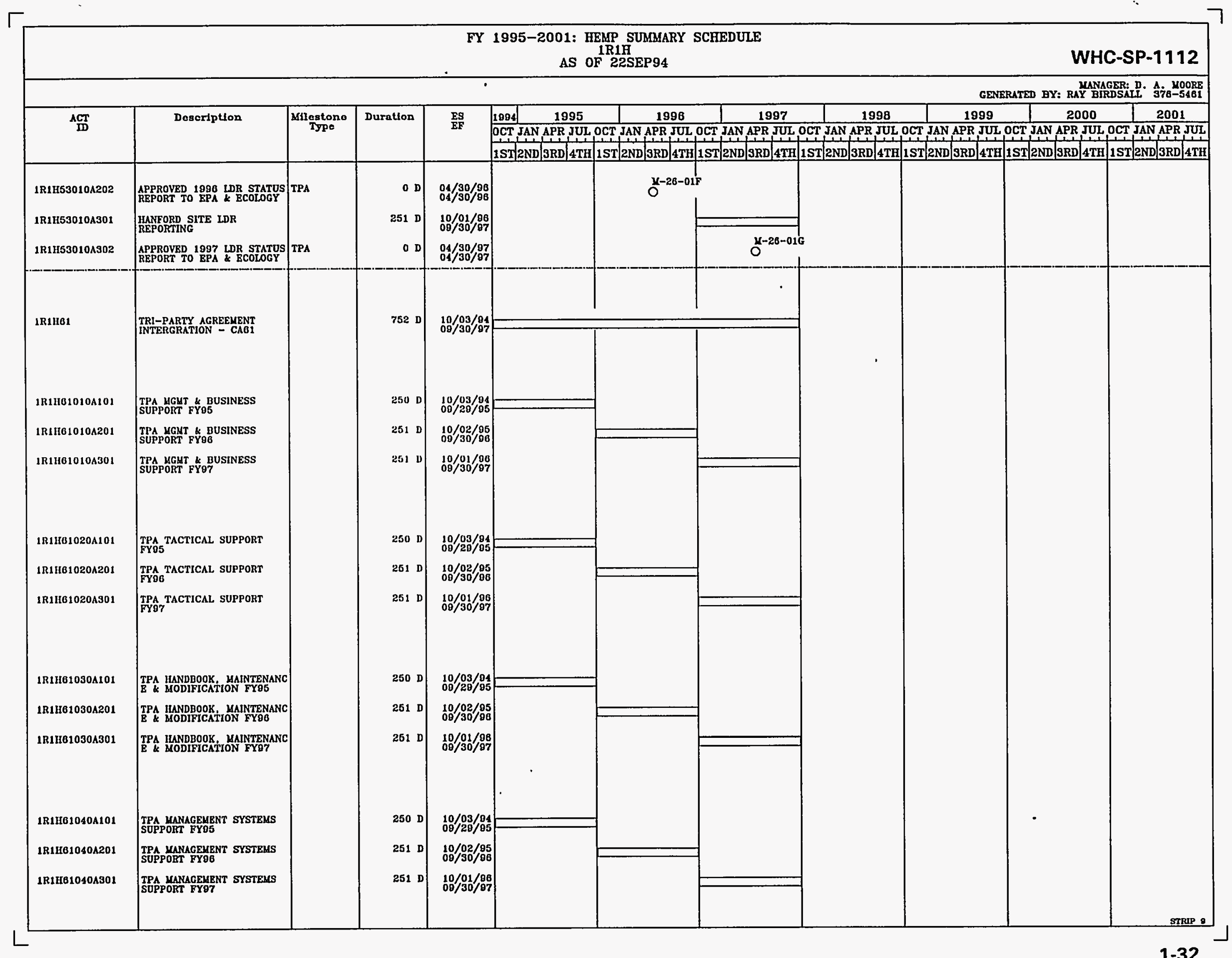




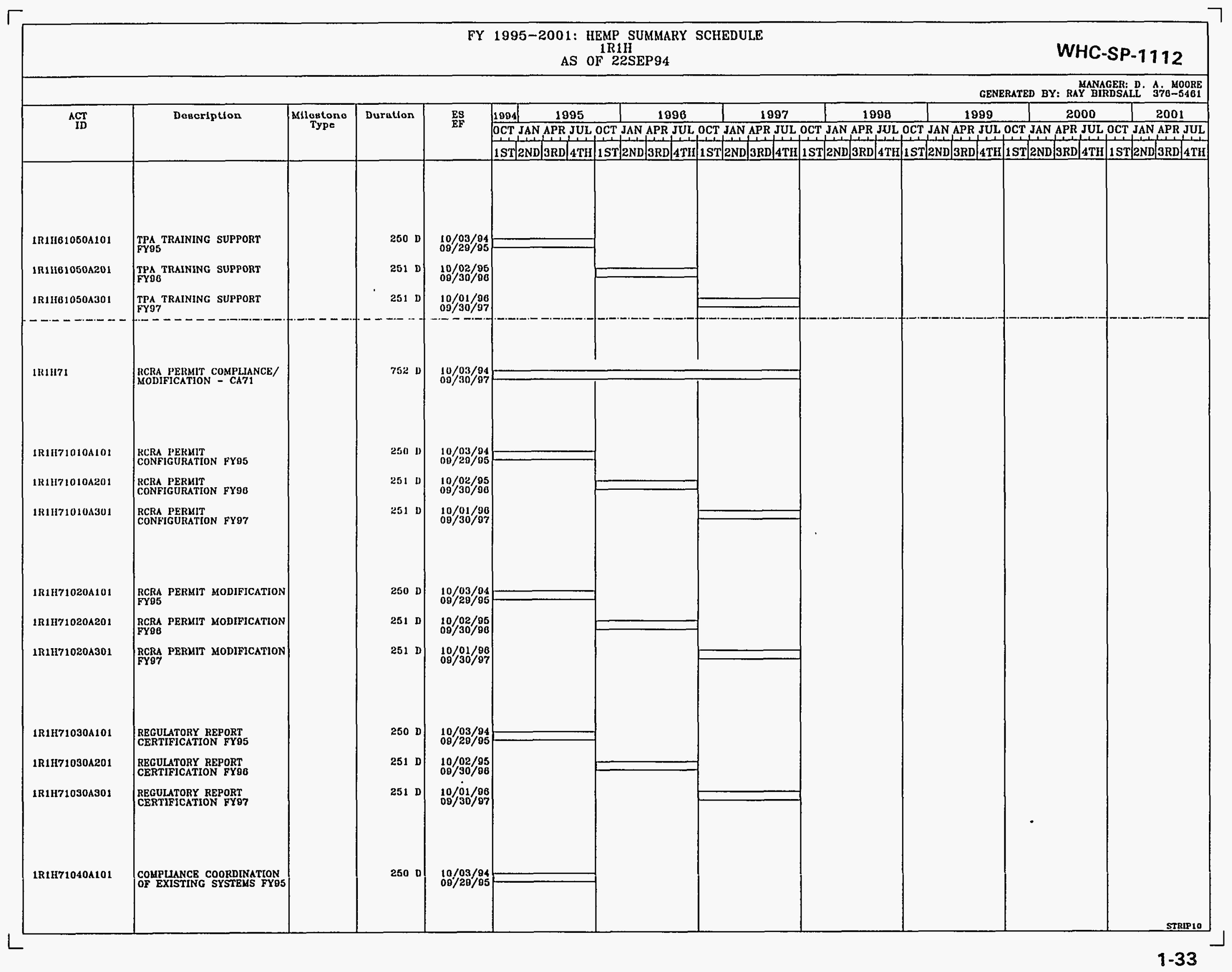




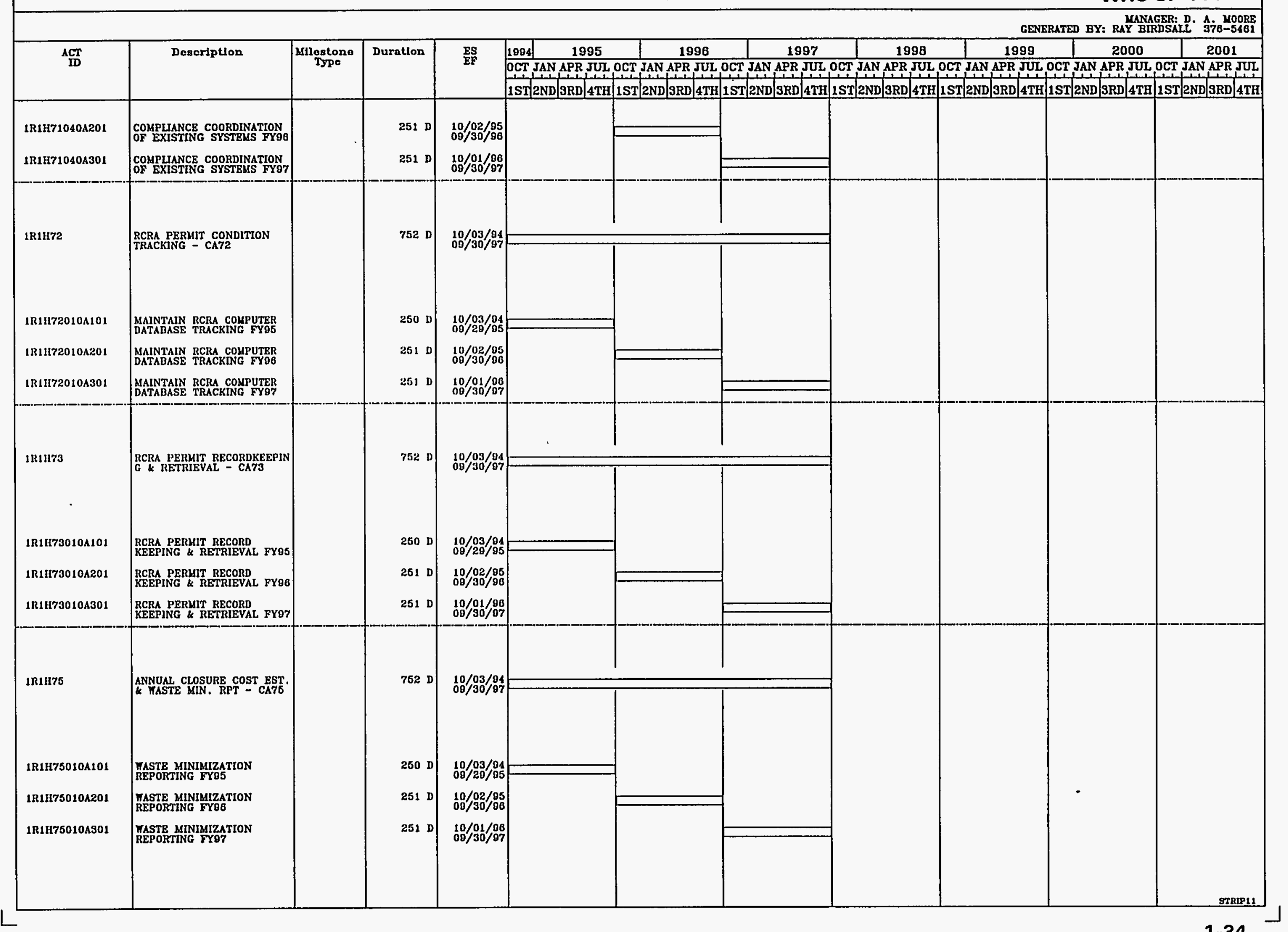




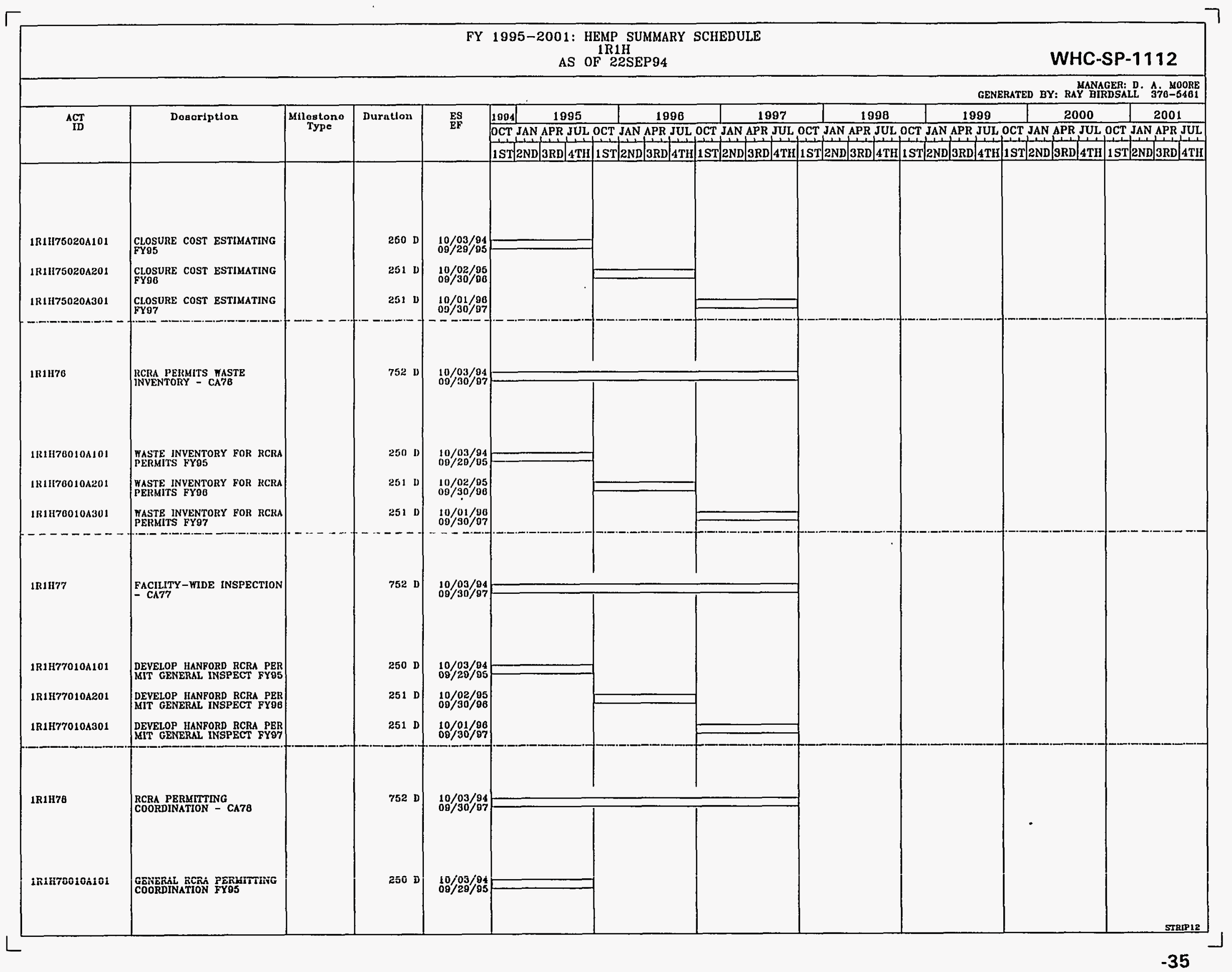




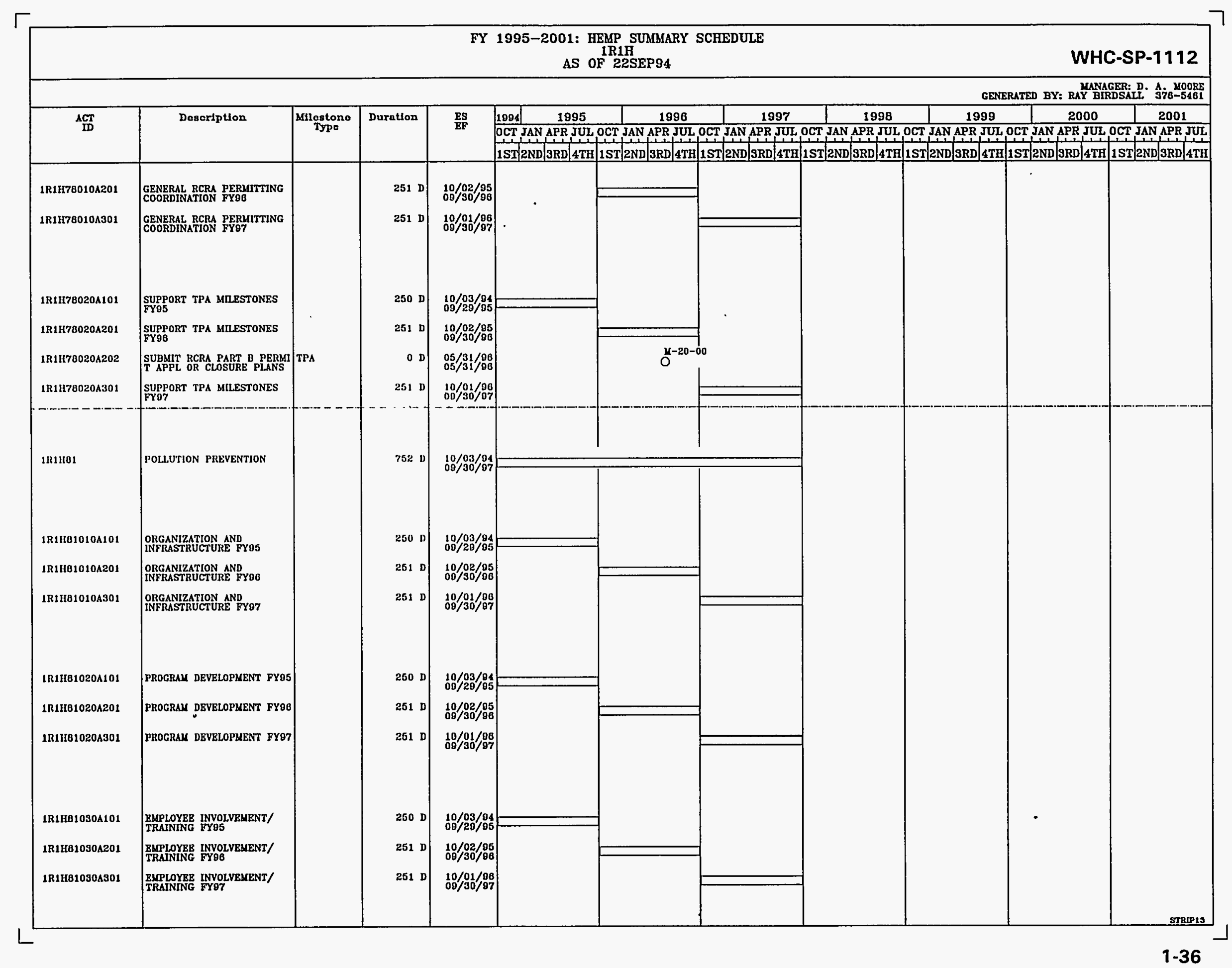




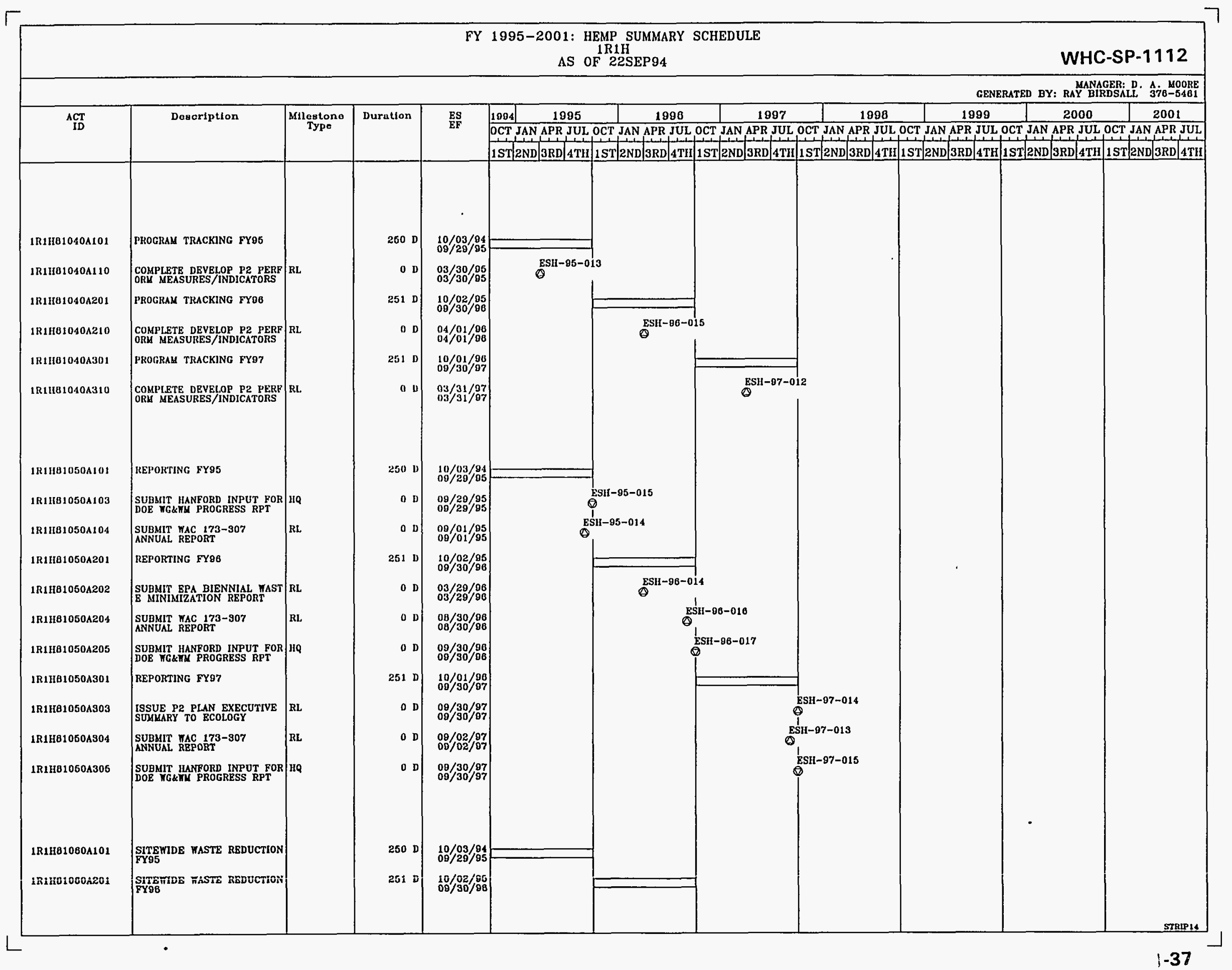




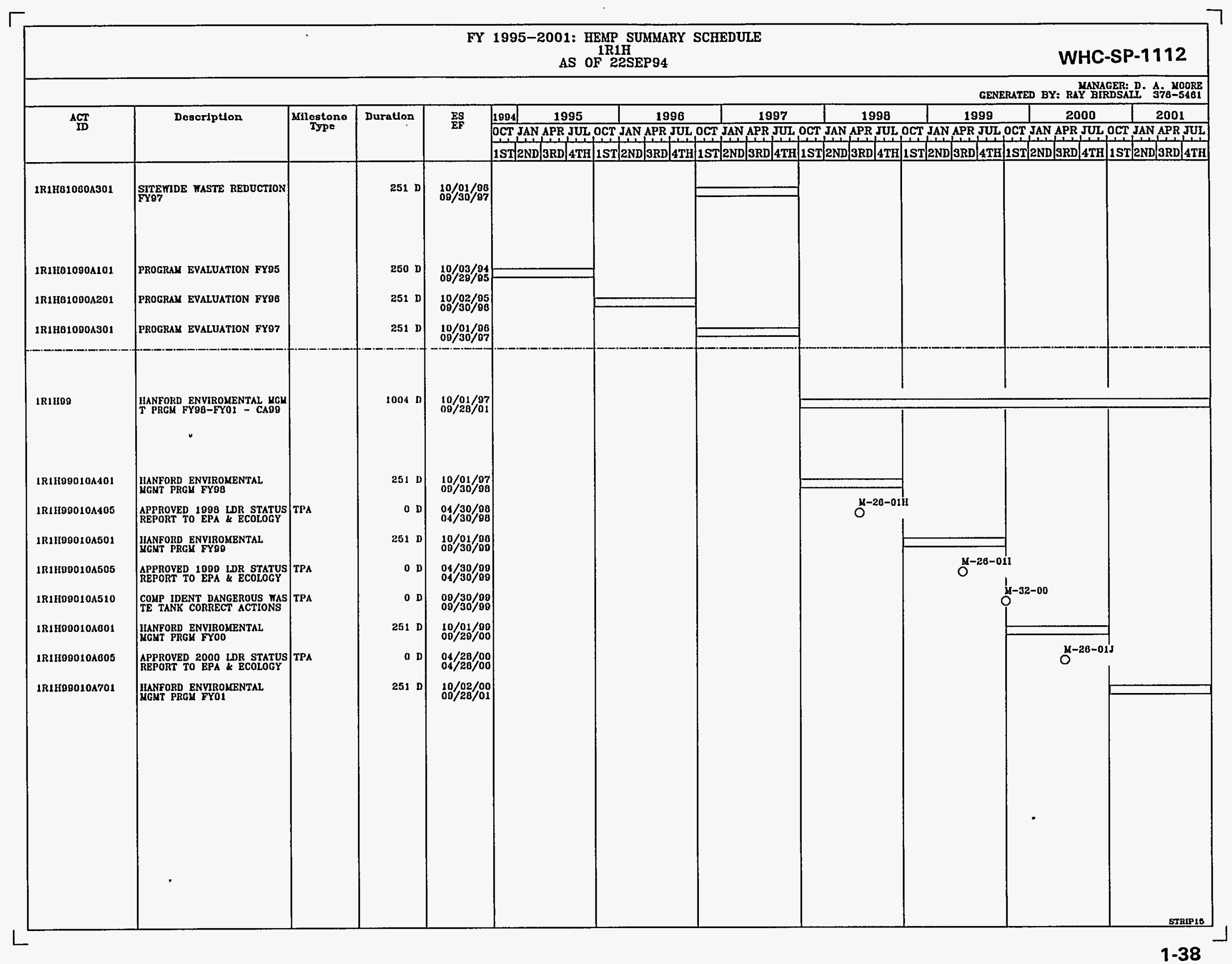


FY 1995 MYPP

\section{E. Program Performance Baseline Schedule (PPBS)}

See attached. 
WHC-SP-1112

FY 1995-1997: PROGRAM ADMINISTRATION

$1 \mathrm{R} 1 \mathrm{H} 11$

AS OF 29AUG94

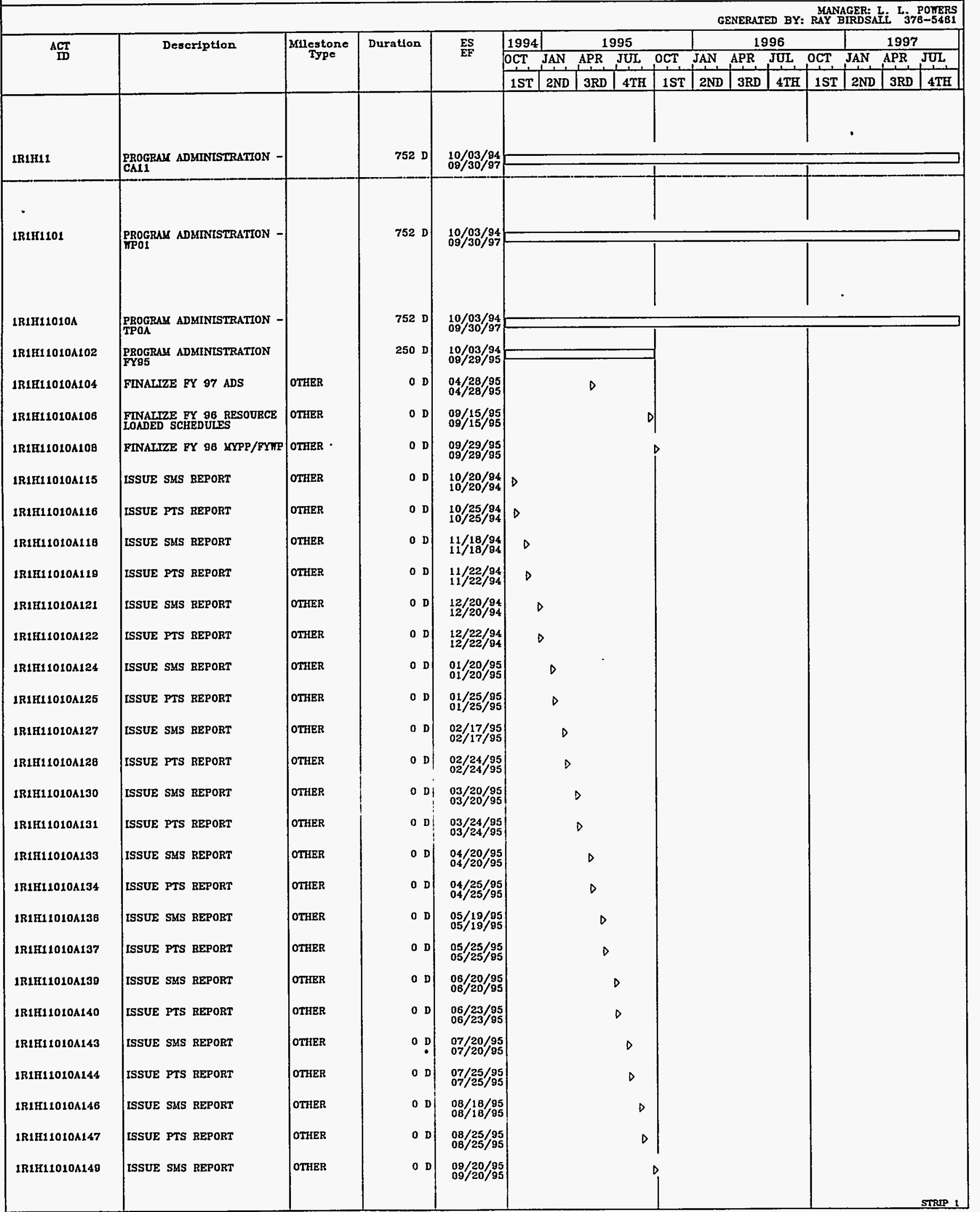


FY 1995-1997: PROGRAM ADMINISTRATION AS OF 29AUG94

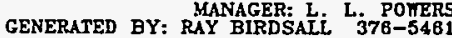

\begin{tabular}{|c|c|c|c|c|c|c|c|c|c|c|c|c|c|c|c|c|}
\hline \multirow{3}{*}{ IDT } & \multirow[t]{3}{*}{ Description } & \multirow{3}{*}{$\underset{\substack{\text { Milestone } \\
\text { Type }}}{ }$} & \multirow[t]{3}{*}{ Duration } & \multirow{3}{*}{$\begin{array}{l}\text { ES } \\
\text { EF }\end{array}$} & 1994 & \multicolumn{4}{|c|}{1995} & \multicolumn{4}{|c|}{1996} & \multicolumn{3}{|c|}{1997} \\
\hline & & & & & OCT & JAN & $\mathrm{APR}$ & JUL & $\mathrm{OCT}$ & JAN & $\mathrm{APR}$ & JUL & OCT & JAN & APR & JUL \\
\hline & & & & & $1 \mathrm{ST}$ & 2ND & 3RD & $4 \mathrm{TH}$ & 1ST & 2ND & 3RD & $4 \mathrm{TH}$ & $1 \mathrm{ST}$ & 2ND & 3ED & $4 \mathrm{TH}$ \\
\hline IRIH11010A15O & ISSUE PTS REPORT & OTHER & $\begin{array}{lll}0 & \mathrm{D}\end{array}$ & $\begin{array}{l}09 / 25 / 95 \\
09 / 25 / 95\end{array}$ & & & & & D & & & & & & & \\
\hline 1RIH11010A202 & $\begin{array}{l}\text { PROGRAM ADMINISTRATION } \\
\text { YY9B }\end{array}$ & & $251 \mathrm{D}$ & $\begin{array}{l}10 / 02 / 95 \\
09 / 30 / 96\end{array}$ & & & & & & & & & & & & \\
\hline 1R1F11010A204 & FINAIIZE FY 98 ADS & OTHER & $0 \mathrm{D}$ & $\begin{array}{l}04 / 30 / 96 \\
04 / 30 / 96\end{array}$ & & & & & & & $D$ & & & & & \\
\hline 1R1H1 1010A208 & $\begin{array}{l}\text { FINALIZE FY } 97 \text { RESOURCE } \\
\text { LOADED SCHEDULES }\end{array}$ & OTHER & $0 \mathrm{D}$ & $\begin{array}{l}09 / 13 / 96 \\
09 / 13 / 96\end{array}$ & & & & & & & & $D$ & $\mid$ & & & \\
\hline 1R1H11010A208 & FINALIZE FY 97 KYPP/FYTP & OTHER & $\begin{array}{lll}0 & \mathrm{D}\end{array}$ & $\begin{array}{l}09 / 30 / 96 \\
09 / 30 / 96\end{array}$ & & & & & & & & & $D$ & & & \\
\hline IR1H11010A215 & ISSUE SMS REPORT & OTHER & $\begin{array}{lll}0 & \mathrm{D}\end{array}$ & $\begin{array}{l}10 / 20 / 95 \\
10 / 20 / 95\end{array}$ & & & & & $D$ & & & & & & & \\
\hline 1R1H11010A218 & ISSUE PTS REPORT & OTHER & $\begin{array}{lll}0 & \mathrm{D}\end{array}$ & $\begin{array}{l}10 / 25 / 95 \\
10 / 25 / 95\end{array}$ & & & & & $D$ & & & & & & & \\
\hline IR1H11010A218 & ISSUE SMS REPORT & OTHER & $0 \mathrm{D}$ & $\begin{array}{l}11 / 20 / 95 \\
11 / 20 / 95\end{array}$ & & & & & $D$ & & & & & & & \\
\hline 1R1H11010A219 & ISSUE PTS REPORT & OTHER & $\begin{array}{lll}0 & \mathrm{D}\end{array}$ & $\begin{array}{l}11 / 22 / 95 \\
11 / 22 / 95\end{array}$ & & & & & $D$ & & & & & & & \\
\hline 1R1H11010A221 & ISSUE SMS REPORT & OTHER & $\begin{array}{lll}0 & 0\end{array}$ & $\begin{array}{l}12 / 20 / 95 \\
12 / 20 / 95\end{array}$ & & & & & D & 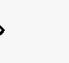 & & & & & & \\
\hline IRIH11010A222 & ISSUE PTS REPORT & OTHER & $0 \mathrm{D}$ & $\begin{array}{l}12 / 21 / 95 \\
12 / 21 / 95\end{array}$ & & & & & D & 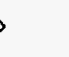 & & & & & & \\
\hline 1R1H11010A224 & ISSUE SMS REPORT & OTHER & $\begin{array}{lll}0 & D\end{array}$ & $\begin{array}{l}01 / 19 / 96 \\
01 / 19 / 96\end{array}$ & & & & & & $D$ & & & & & & \\
\hline 1R1H11010A225 & ISSUE PTS REPORT & OTHER & $0 \mathrm{D}$ & $\begin{array}{l}01 / 25 / 96 \\
01 / 25 / 96\end{array}$ & & & & & & $D$ & & & & & & \\
\hline 1R1H11010A227 & ISSUE SMS REPORT & OTHER & $O D$ & $\begin{array}{l}02 / 20 / 96 \\
02 / 20 / 96\end{array}$ & & & & & & $D$ & & & & & & \\
\hline 1R1H11010A228 & ISSUE PTS REPORT & OTHER & $\begin{array}{lll}0 & \mathrm{D}\end{array}$ & $\begin{array}{l}02 / 23 / 96 \\
02 / 23 / 96\end{array}$ & & & & & & $D$ & & & & & & \\
\hline IRIH11010A230 & ISSUE SMS REPORT & OTHER & $O D$ & $\begin{array}{l}03 / 20 / 96 \\
03 / 20 / 96\end{array}$ & & & & & & & $\triangleright$ & & & & & \\
\hline 1R1F11010A231 & ISSUE PTS REPORT & OTHER & $0 \mathrm{D}$ & $\begin{array}{l}03 / 25 / 96 \\
03 / 25 / 96\end{array}$ & & & & & & & $\triangleright$ & & & & & \\
\hline 1R1K11010A233 & ISSUE SMS REPORT & OTHER & $0 \quad \mathrm{D}$ & $\begin{array}{l}04 / 19 / 96 \\
04 / 19 / 96\end{array}$ & & & & & & & $D$ & & & & & \\
\hline IR1H11010A234 & ISSUE PTS REPORT & OTHER & $0 \mathrm{D}$ & $\begin{array}{l}04 / 25 / 96 \\
04 / 25 / 96\end{array}$ & & & & & & & $\triangleright$ & & & & & \\
\hline IR1E11010A236 & ISSUE SMS REPORT & OTHER & $O D$ & $\begin{array}{l}05 / 20 / 96 \\
05 / 20 / 96\end{array}$ & & & & & & & $D$ & & & & & \\
\hline IR1E11010A237 & ISSUE PTS REPORT & OTHER & O D & $\begin{array}{l}05 / 24 / 96 \\
05 / 24 / 96\end{array}$ & & & & & & & $D$ & & & & & \\
\hline IR1H11010A239 & ISSUE SMS REPORT & OTHER & o $\mathbf{v}$. & $\begin{array}{l}06 / 20 / 96 \\
06 / 20 / 96\end{array}$ & & & & & & & & D & & & & \\
\hline IRIH11010A240 & ISSUE PTS REPORT & OTHER & O D : & $\begin{array}{l}06 / 25 / 98 \\
06 / 25 / 96\end{array}$ & & & & & & & & $D$ & & & & \\
\hline IR1K11O1OA243 & ISSUE SMS REPORT & OTHER & $O D$ & $\begin{array}{l}07 / 19 / 96 \\
07 / 19 / 96\end{array}$ & & & & & & & & $D$ & & & & \\
\hline IR1E11010A244 & ISSUE PTS REPORT & OTHER & $0 \mathrm{D}$ & $\begin{array}{l}07 / 25 / 96 \\
07 / 25 / 96\end{array}$ & & & & & & & & $\nabla$ & & & & \\
\hline 1R1H11010A246 & ISSUE SMS REPORT & OTHER & 00 & $\begin{array}{l}08 / 20 / 96 \\
08 / 20 / 96\end{array}$ & & & & & & & & $D$ & & & & \\
\hline IR1H11010A247 & ISSUE PTS REPORT & OTHER & $O \mathrm{D}$ & $\begin{array}{l}08 / 23 / 96 \\
08 / 23 / 96\end{array}$ & & & & & & & & $D$ & & & & \\
\hline 1R1H11010A249 & ISSUE SMS REPORT & OTHER & $0 \mathrm{D}$ & $\begin{array}{l}09 / 20 / 96 \\
09 / 20 / 96\end{array}$ & & & & & & & & $D_{1}$ & i & & & \\
\hline 1R1F11010A250 & ISSUE PTS REPORT & OTHER & $0 \mathrm{D}$ & $\begin{array}{l}09 / 25 / 96 \\
09 / 25 / 98\end{array}$ & & & & & & & & & 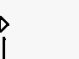 & & & \\
\hline IR1H11010A302 & $\begin{array}{l}\text { PROGRAM ADMINISTRATION } \\
\text { FY97 }\end{array}$ & & 2510 & $\begin{array}{l}10 / 01 / 96 \\
09 / 30 / 97\end{array}$ & & & & & & & & & & & & \\
\hline 1R1H11010A904 & FINALIZE FY 90 ADS & OTHER & 00 & $\begin{array}{l}04 / 30 / 97 \\
04 / 30 / 97\end{array}$ & & & & & & & & & & & $D$ & \\
\hline 1R1H11010A308 & $\begin{array}{l}\text { FINALIZE FY 98 RESOURCE } \\
\text { IOADED SCHEDULES }\end{array}$ & OTHER & 0 D & $\begin{array}{l}09 / 15 / 97 \\
09 / 15 / 97\end{array}$ & & & & & & & & & & & & $D$ \\
\hline IR1H11010A308 & FINALIZE FY 98 XYPP/FYTP & OTHER & $\begin{array}{lll}0 & 0\end{array}$ & $\begin{array}{l}09 / 30 / 97 \\
09 / 30 / 97\end{array}$ & & & & & & & & & & & & o \\
\hline 1R1H11010A315 & ISSUE SMS REPORT & OTHER & 0 o & $\begin{array}{l}10 / 18 / 96 \\
10 / 18 / 96\end{array}$ & & & & & & & & & $D$ & & & \\
\hline
\end{tabular}


FY 1995-1997: PROGRAM ADMINISTRATION

1R1H11

AS OF 29AUG94

GENERATED BY: RAYAGGER: L. L. POMERS

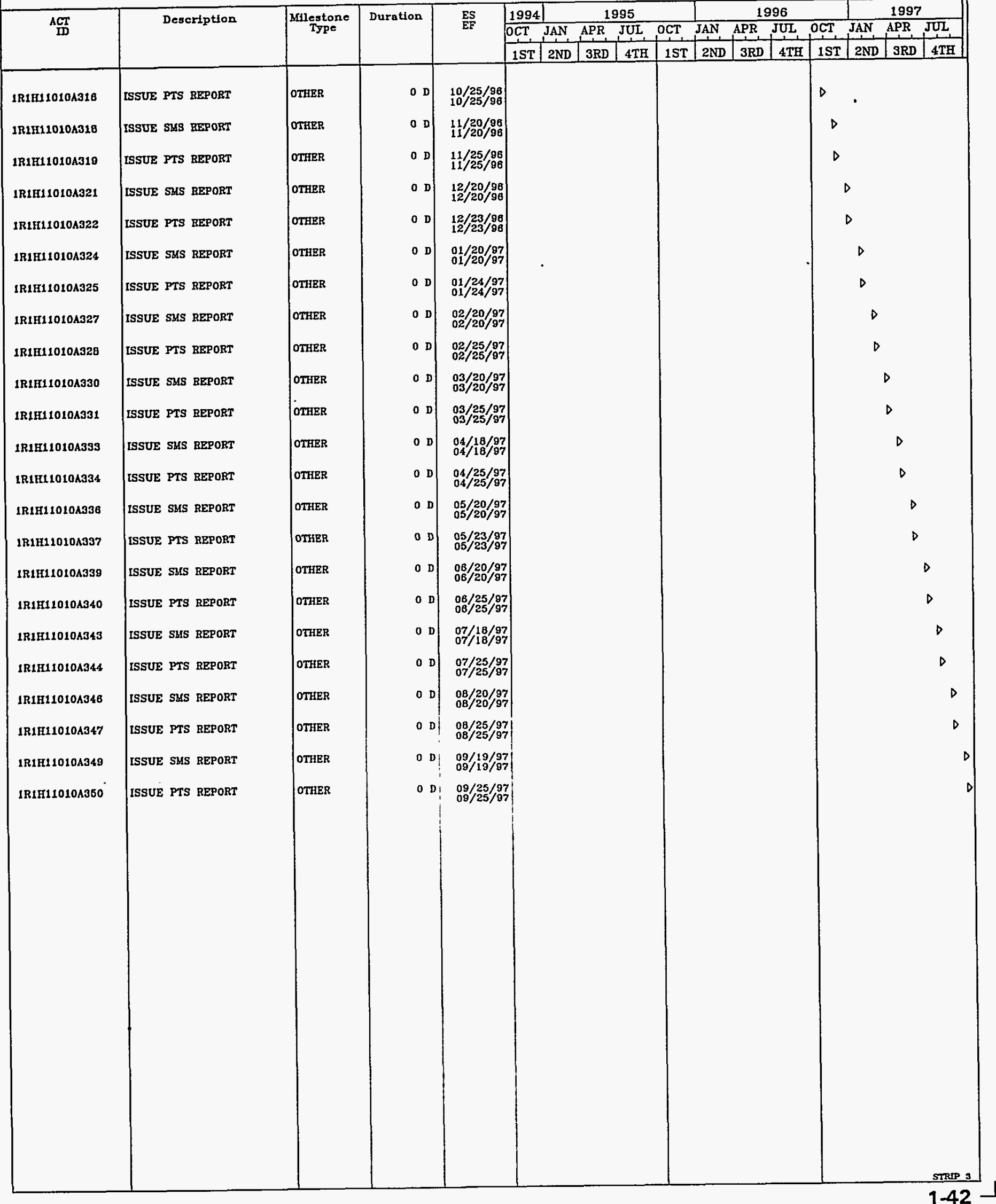




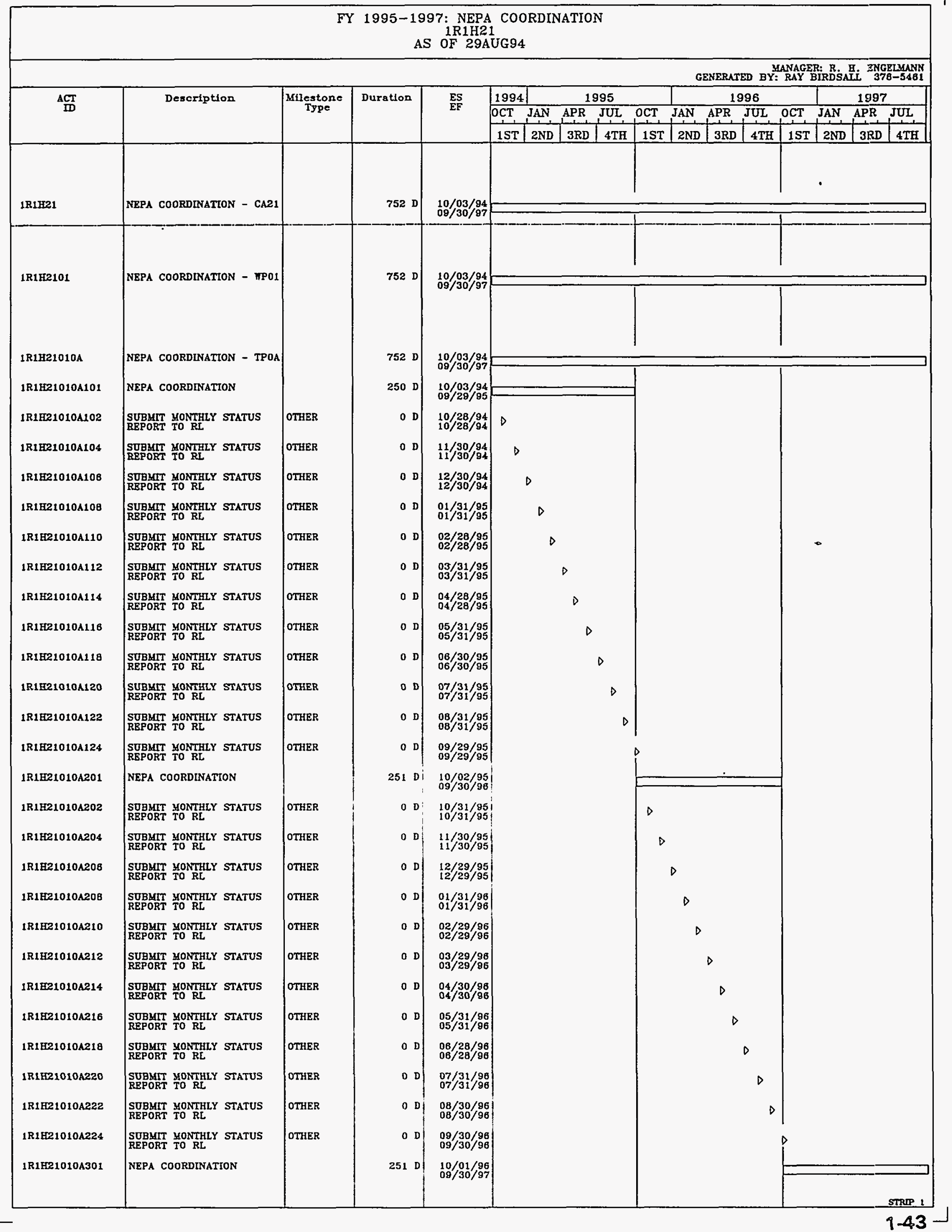




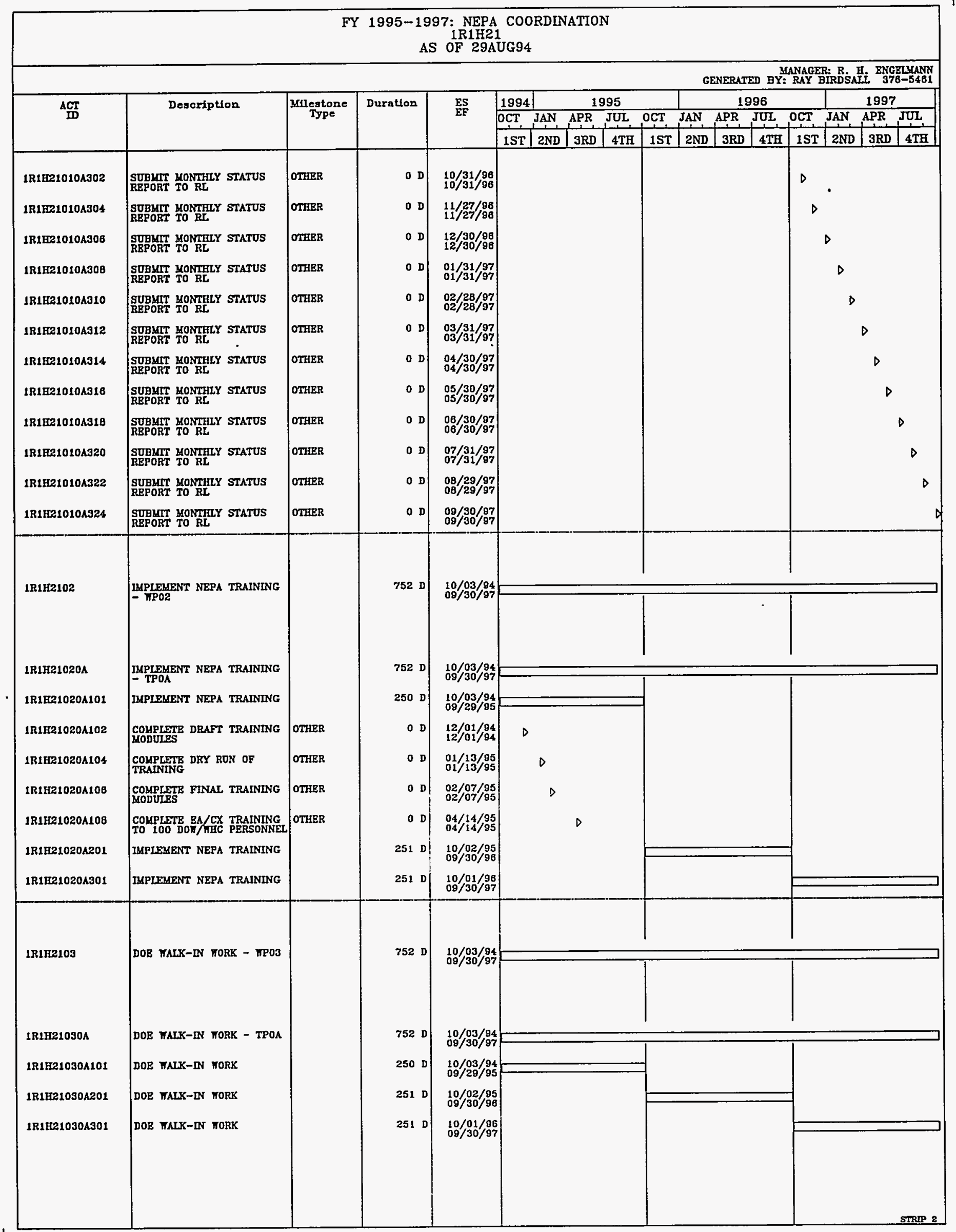




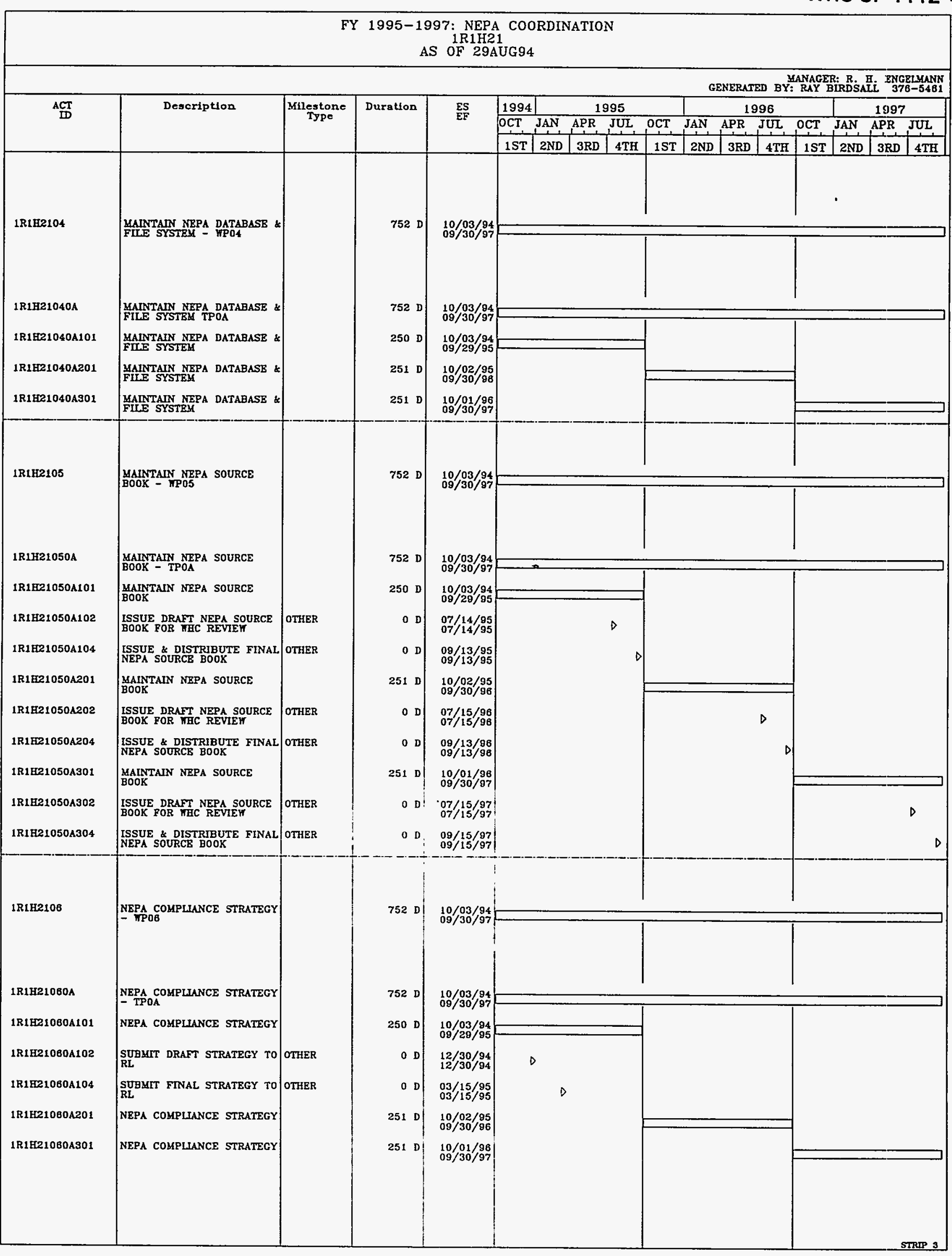


FY 1995-1997: NEPA COORDINATION 1R1H21

AS OF 29AUG94

GENERATED BY: RAY BIRDSAIL ENGELYANN

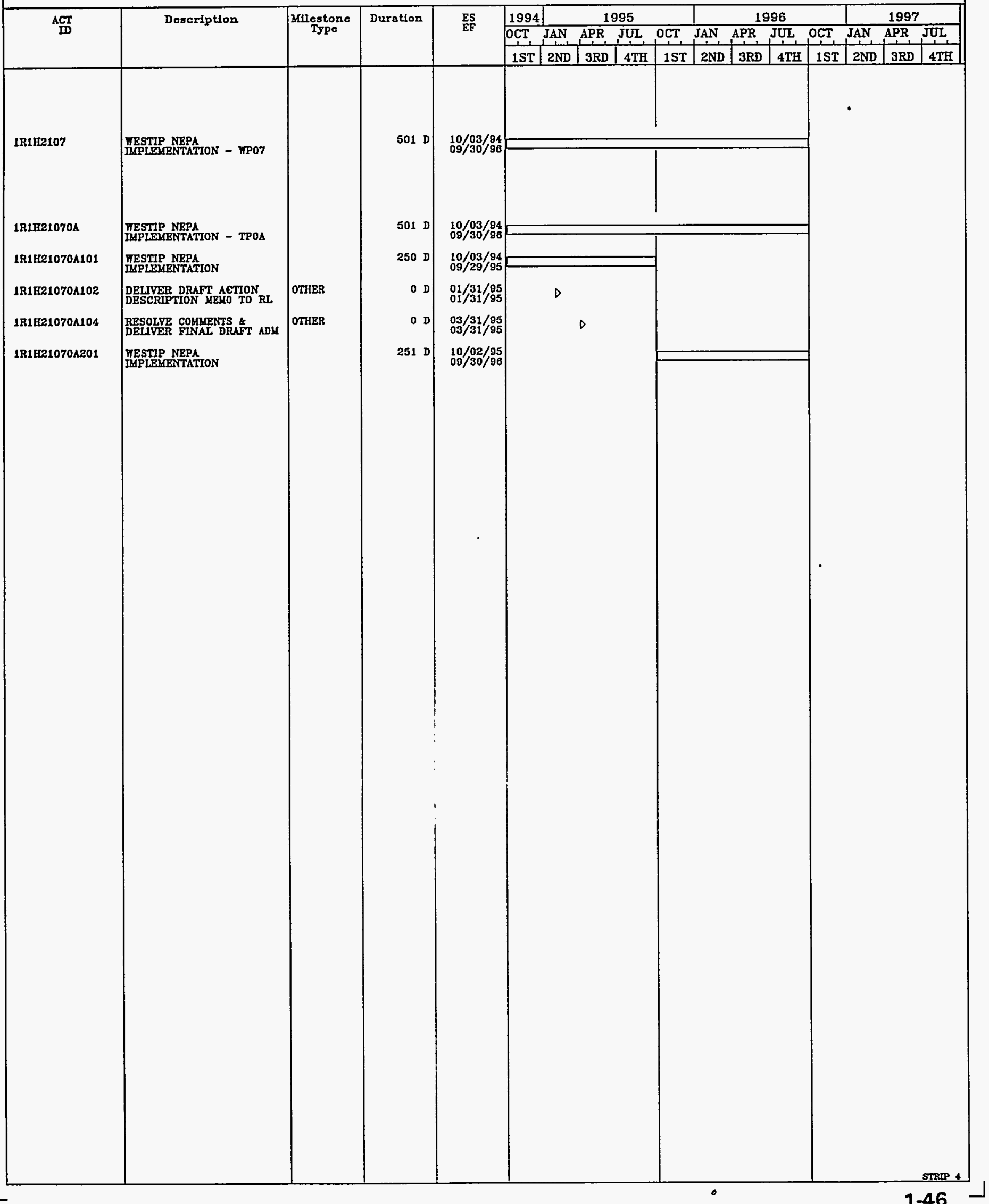


FY 1995-1997: ENVIRONMENTAL FIELD SERVICES 1 R1H31

AS OF 29AUG94

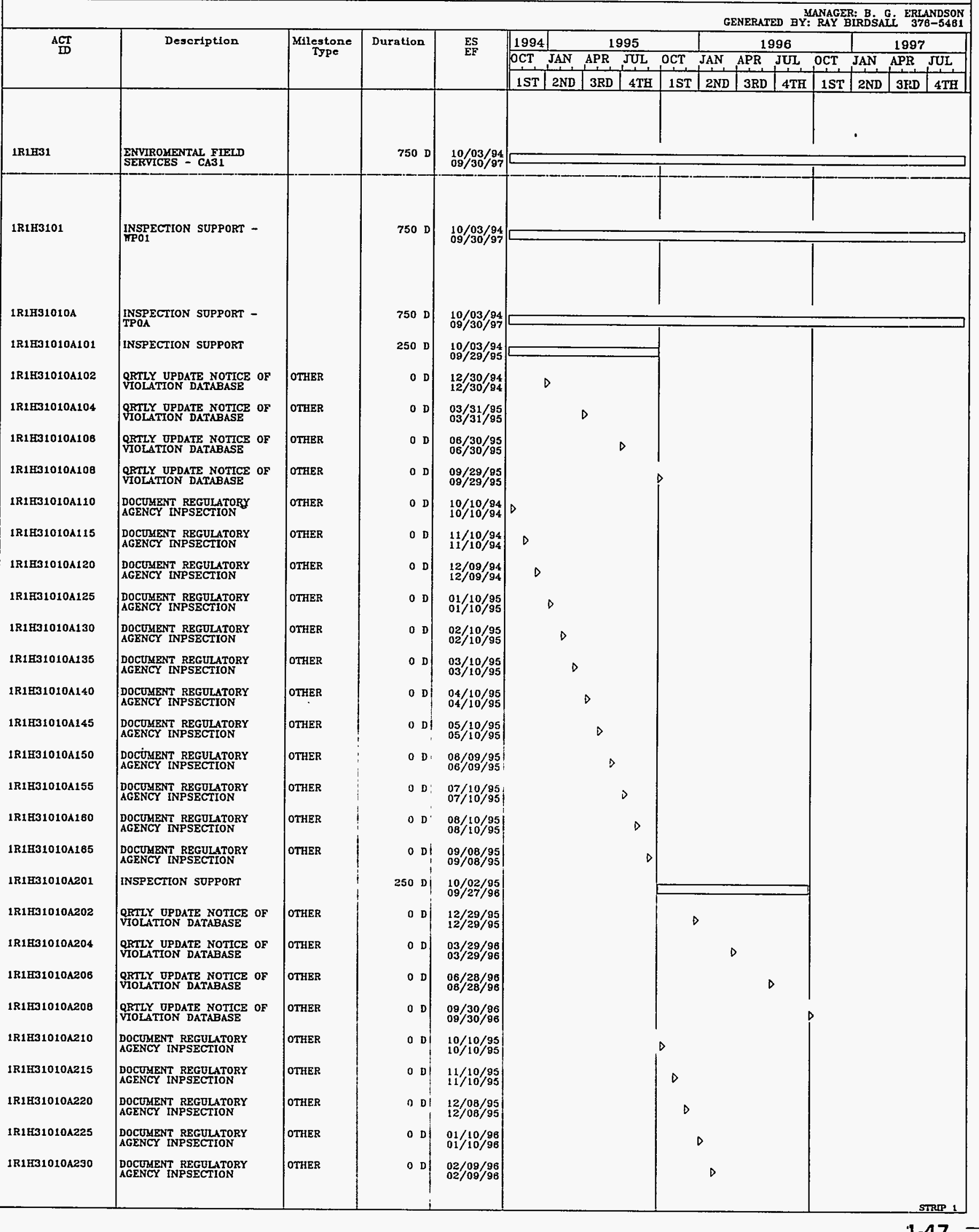


FY 1995-1997: ENVIRONMENTAL FIELD SERVICES

$1 \mathrm{R} 1 \mathrm{H} 31$

AS OF 29AUG94

GENERATED BY: RAYY BR: Bं ${ }^{G}$ GRT BRANDSOM

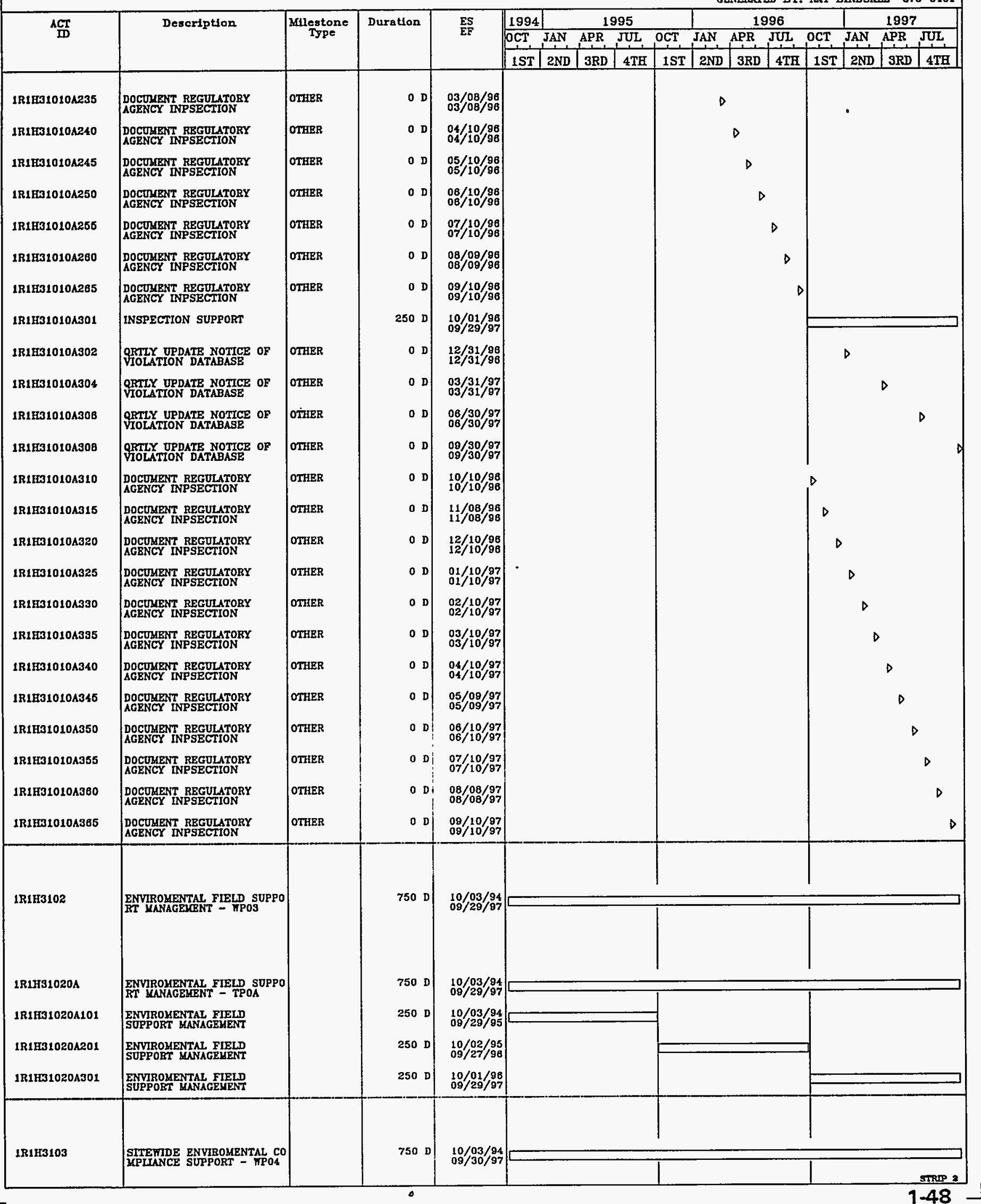


FY 1995-1997: ENVIRONMENTAL FIELD SERVICES

$1 \mathrm{R} 1 \mathrm{H} 31$

AS OF 29AUG94

GENERATED BY: RAY BIRDSALi

SITEFIDE ENVIROMENTAL CO

1R1H3I030A

1R1E31030A101

1R1H310SOA102

IR1E31030A104

IR1E31030A10B

1R1F31030A108

1R1E310SOAL16

IR1H31030AL18

IR1H31030A20

1R1H31030A202

1R1E31030A204

IR1E31030A208

1R1H3103OA208

1R1EB1030A212

IR1H31030N214

1R1H3103OA216

1R1H31030A218

IR1H31030A30

1R1H31030A302

1R1E31030ASO4

1R1E31030A30B

1R1H31030AS08

1R1EM1090A912

1R1H31030A814

1RIE31030A316

1R1H31030A318

\begin{tabular}{l|l} 
ISSUE PRODUCT YGLTT/ \\
RECYCLING CENTER REPORT
\end{tabular}

STTEMTDE ENVROMENTAL
COMPLIANCE SUPPORT

STATUS M-M2 PROGRESS FOR OTHER TPA REVIET MEETING

STATUS Y-32 PROGRESS FOR OTHER STATUS Y Y-32 PROGRESS FOR OTHER STATUS Y-92 PROGRESS FOR OTHER TPA REVIET MEETING

ISSUE PRODUCT YGMT/ RECYCLING CENTER REPORT

ISSUE PRODUCT MGLTT ISSUE PRODUCT YGLTT/ ISSUE PRODTCT YGTT RECYCLING CENTER REPORT SITEHIDE ENVIROMENTAL

STATUS M-32 PROGRESS FOR OTHER TPA REVIET MEETING

STATUS Y-32 PROGRESS FOR OTHER TPA REVIET MEETING

TTATUS M-32 PROGRESS FOR OTHER STATUS Y-32 PROGRESS FOR OTHER TPA REVET MEETING

ISSUE PRODUCT MGST/ RECYCLING CENTER REPORT ISSUE PRODUCT YGIT/ ISSUE PRODUCT MGMTT RECYCLING CENTER REPORT

ISSUE PRODUCT

RECYCLNG CENTER REPORT

STTEWIDE ENVROMENTAL

STATUS $M-32$ PROGRESS FOR OTHER TPA REVIET MEETING

STATUS $4-32$ PROGRESS FOR OTHER TPA REVIEH MEETING

STATUS Y-32 PROGRESS FOR OTHER

STATUS $M-32$ PROGRESS FOR OTHER TPA REVIET HEETING

ISSUE PRODUCT YGGMT/

RECYCLING CENTER REPORT

ISSUE PRODUCT YGATT

RECYCING CENER REPORT

ISSUE PRODUCT YGGTT
RECYCLING CENTER RGPOR

ISSUE PRODUCT YGHT

OTHER
OTHER
OTHER
OTHER

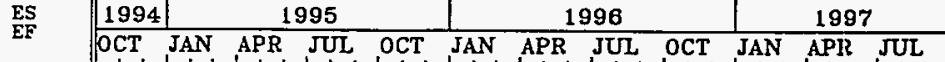
\begin{tabular}{|l|l|l|l|l|l|l|l|l|l|l|l|}
\hline 1ST & 2ND & 3RD & 4TH & 1ST & 2ND & 3RD & 4TH & 1ST & 2ND & 3RD & 4TH \\
\hline
\end{tabular}

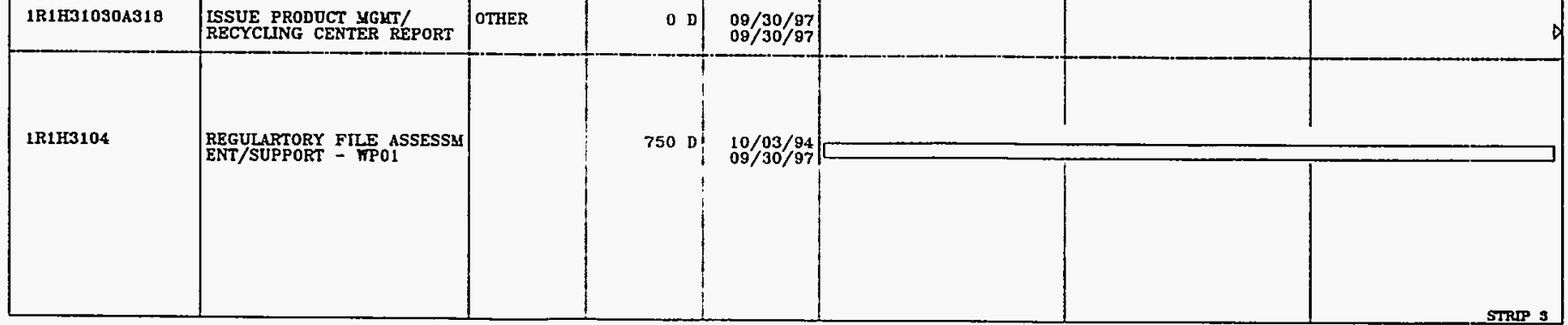


FY 1995-1997: ENVIRONMENTAL FIELD SERVICES

IR1H31

AS OF 29AUG94

GENERATED BY: RAY BRRDSACE

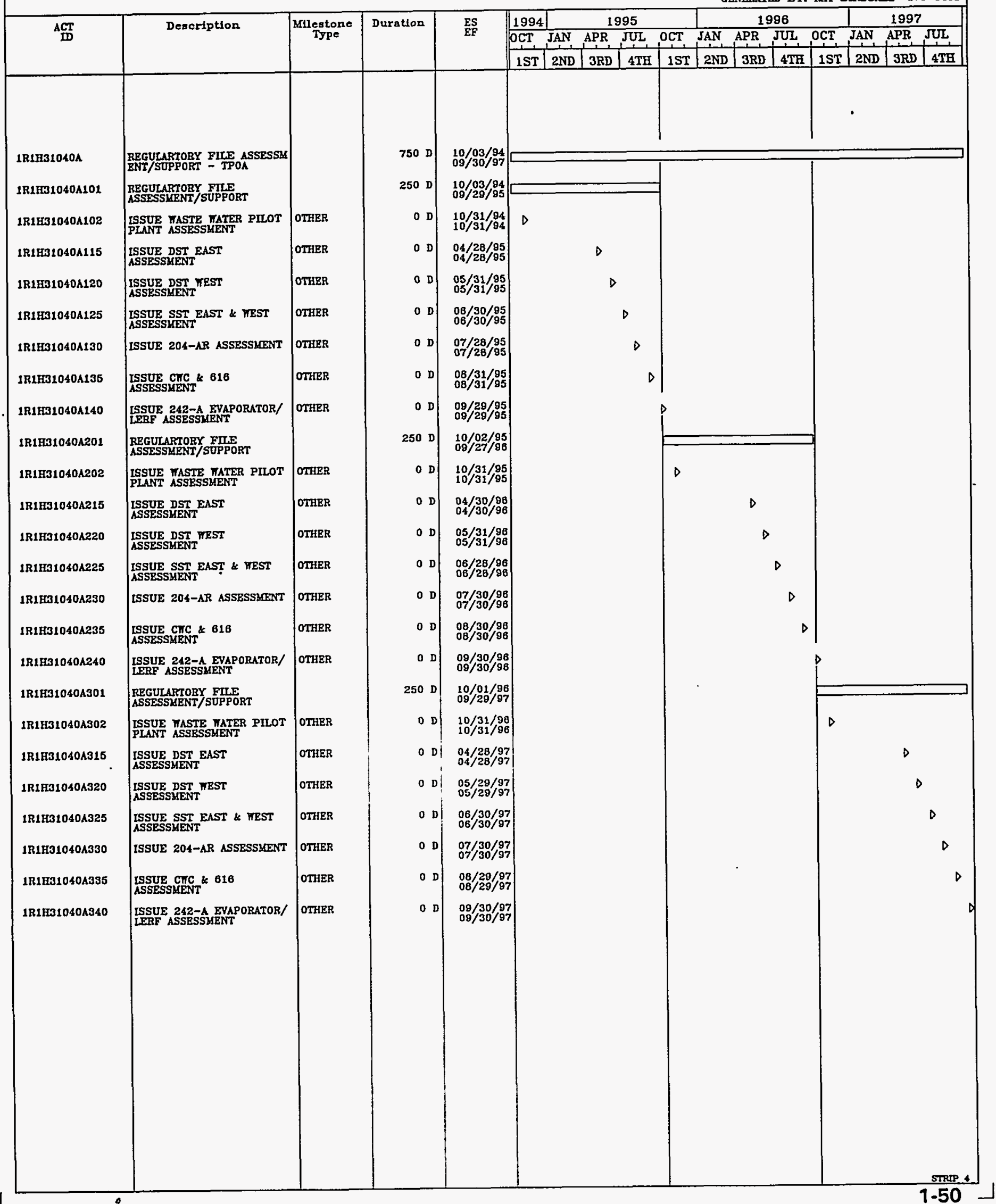


FY 1995-1997: AIR \& WATER PERMITTING COORDINATION 1R1H41

AS OF 29AUG94

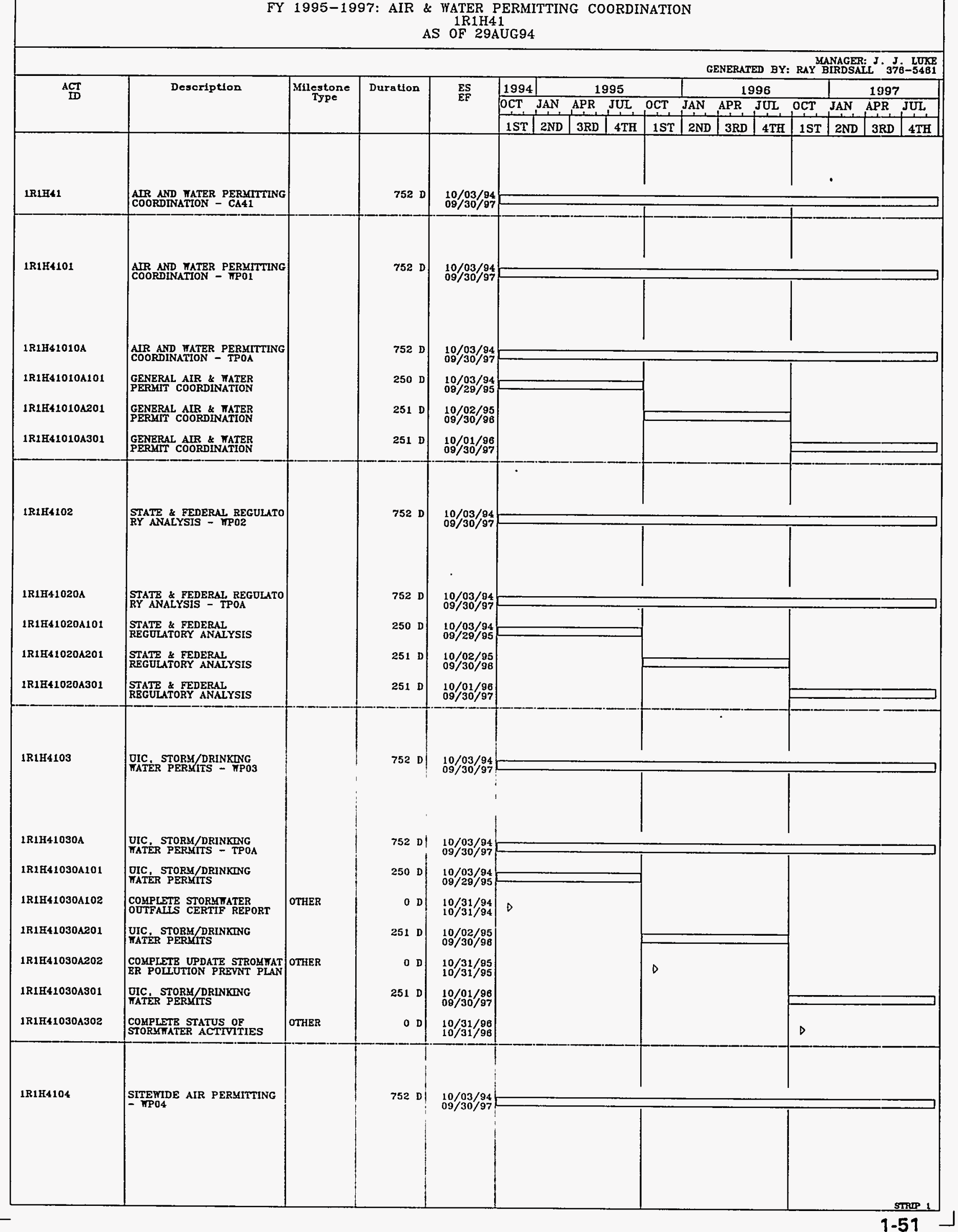




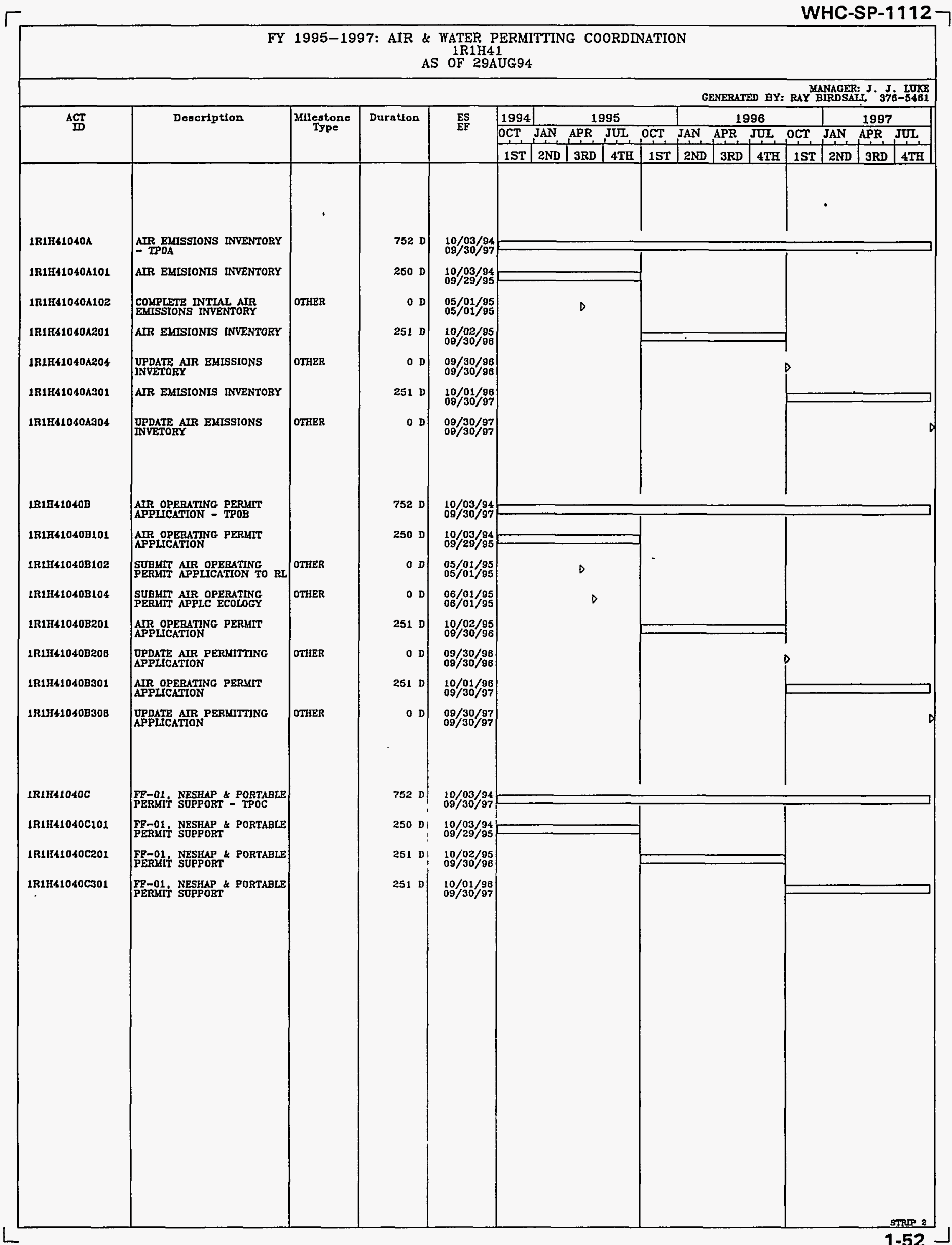




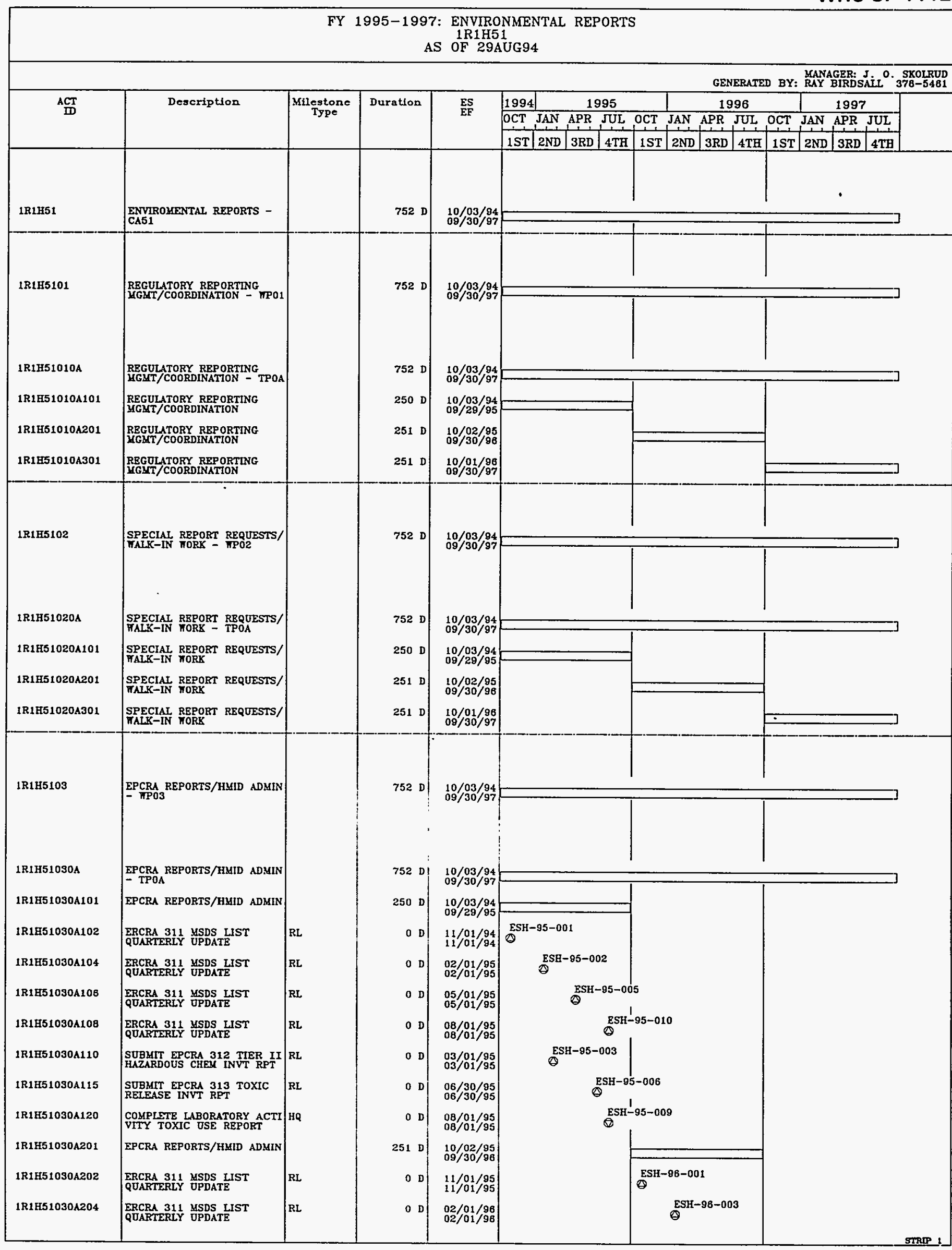


FY 1995-1997: ENVIRONMENTAL REPORTS

IR1H51

AS OF 29AUG94

GENERATED BY: MANAGER: $J{ }^{\circ}{ }^{\circ}$ SKOLRUD

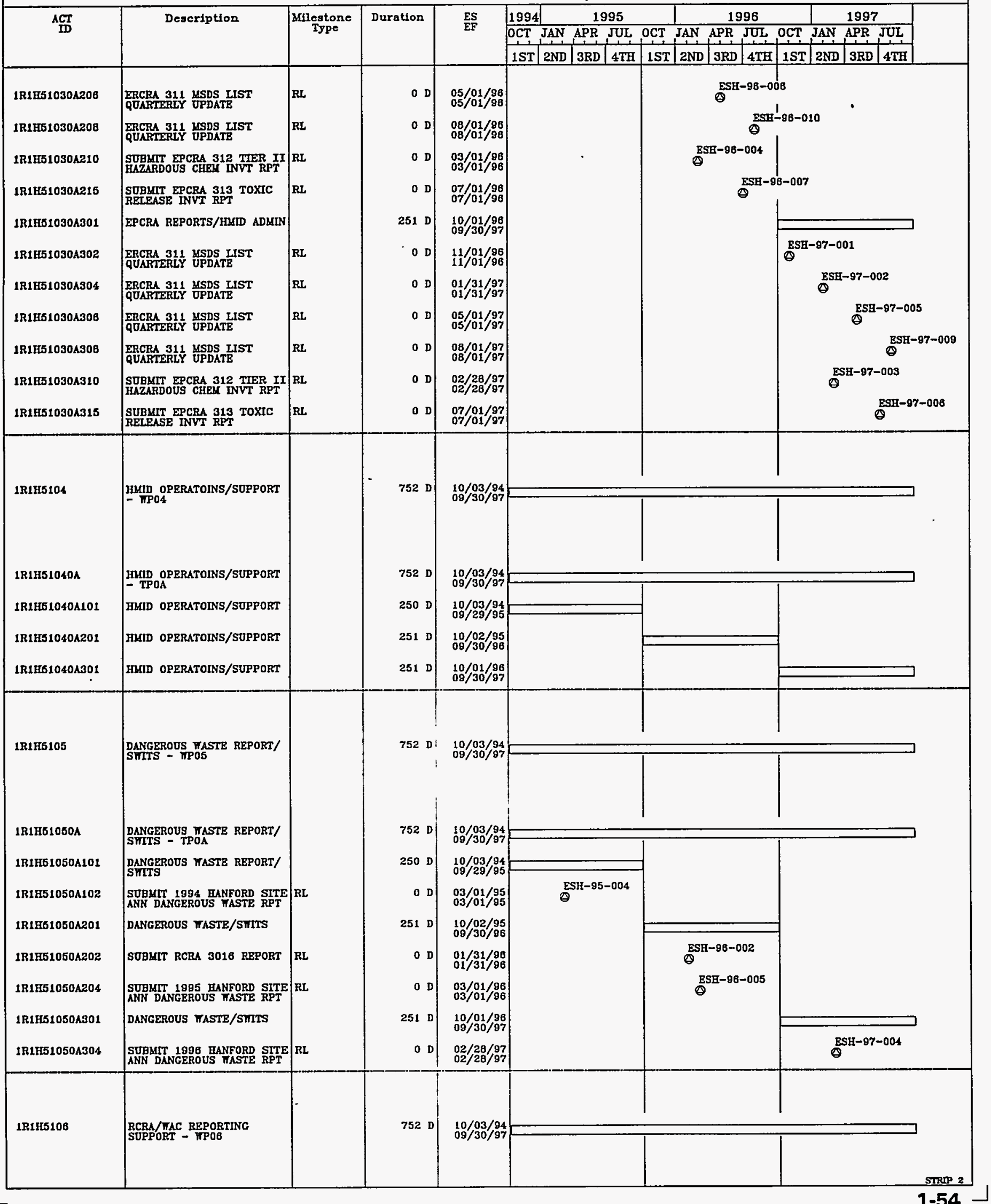




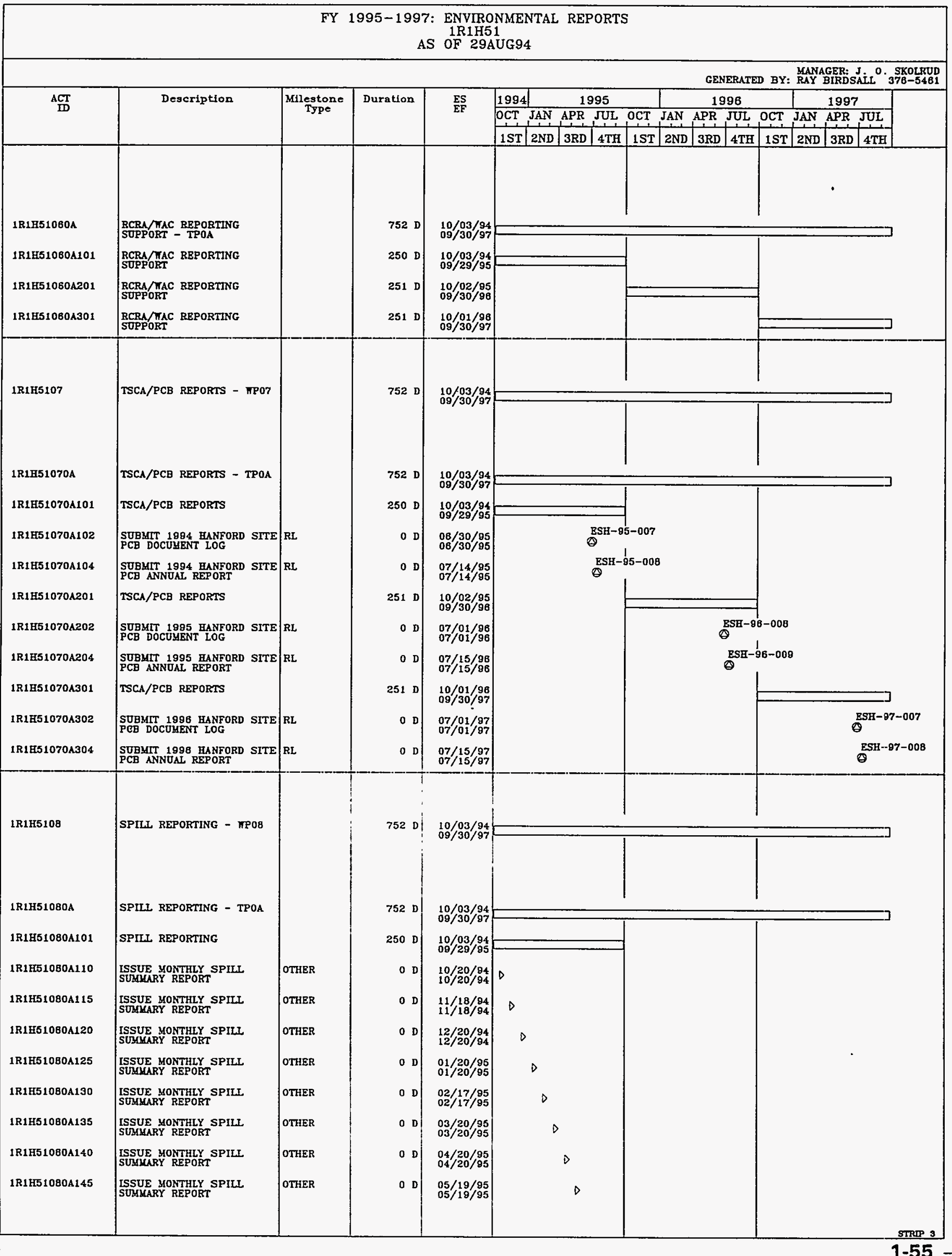


FY 1995-1997: ENVIRONMENTAL REPORTS

$1 \mathrm{R} 1 \mathrm{H} 51$

AS OF 29AUG94

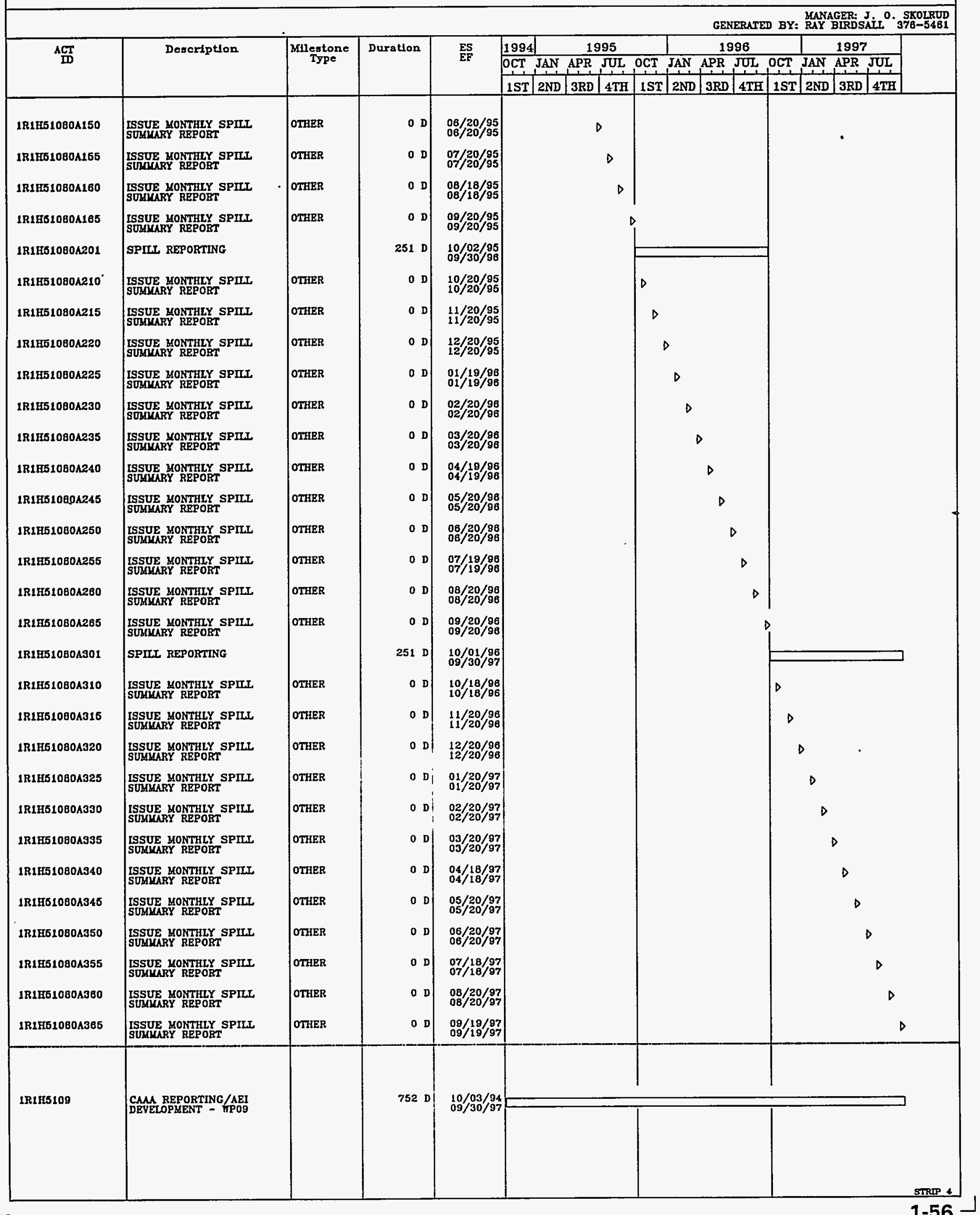



FY 1995-1997: CENRTC-UNIX COMPUTER PROCUREMENT

AS OF 29AUG94

GENERATED BY MANAGER: J 0 . SKOLRUD

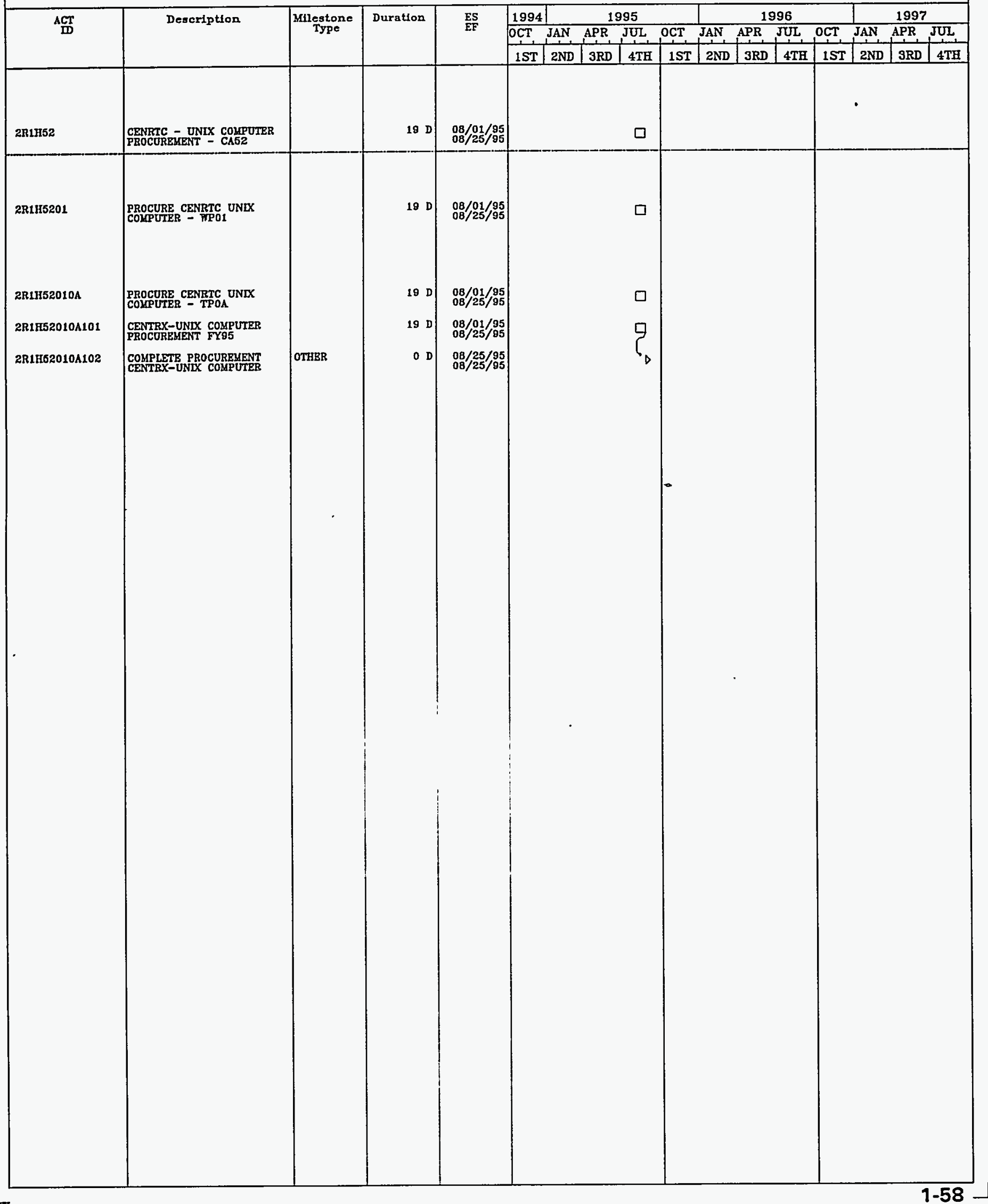




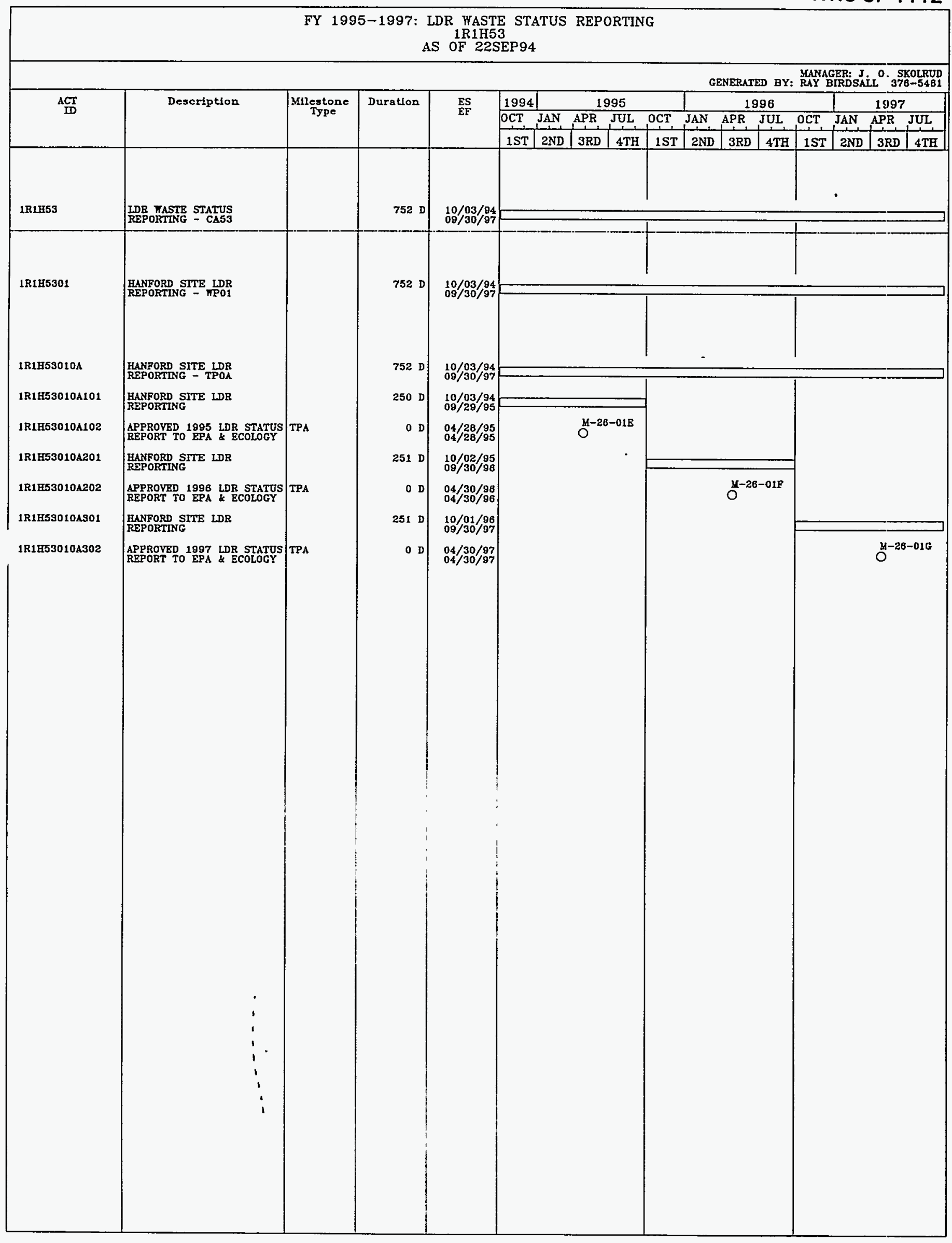


FY 1995-1997: TPA INTEGRATION

1R1H61

AS OF 29AUG94

GENERATED BY: RAY BANAGER: Lt: Do ARNOLID

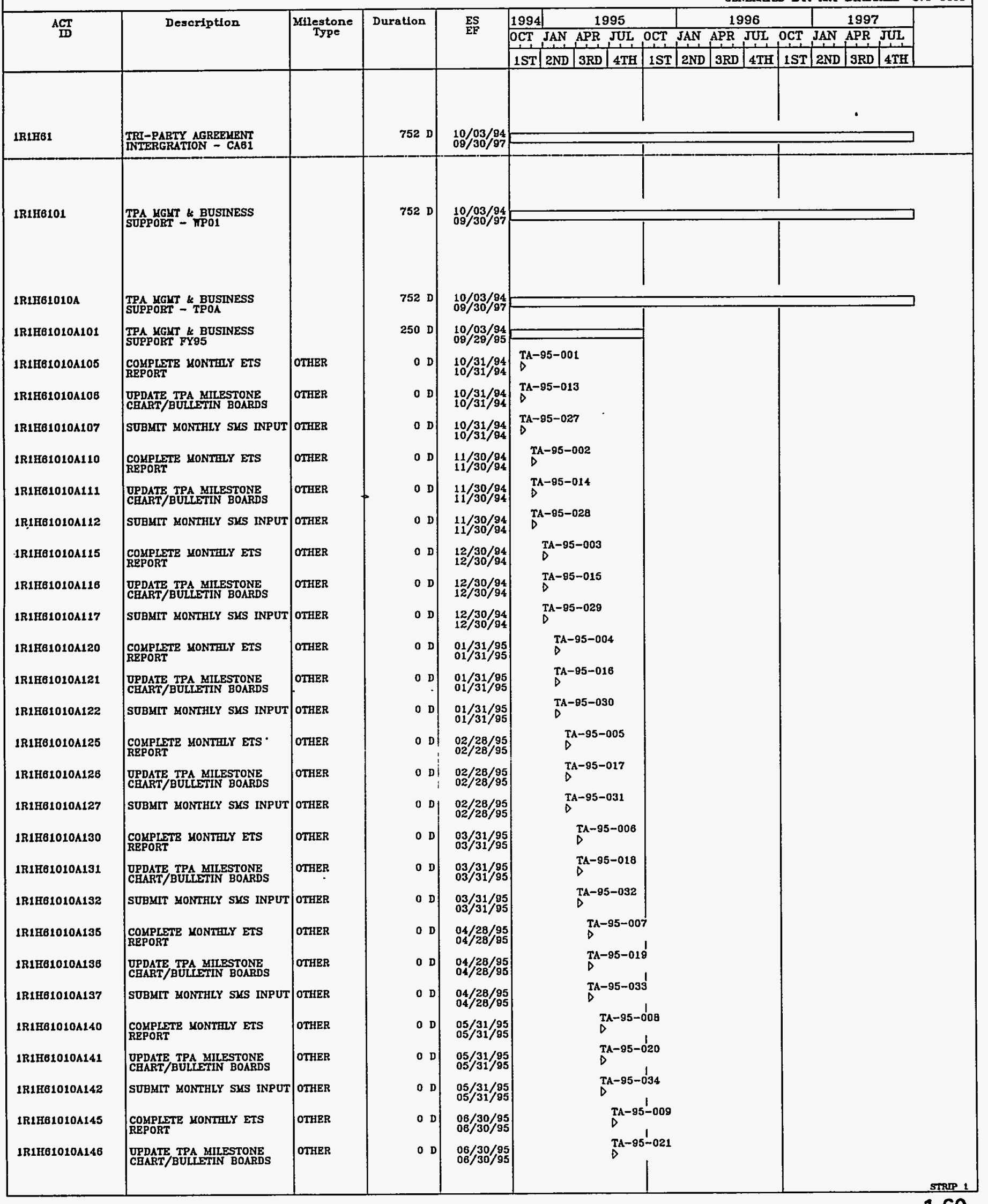




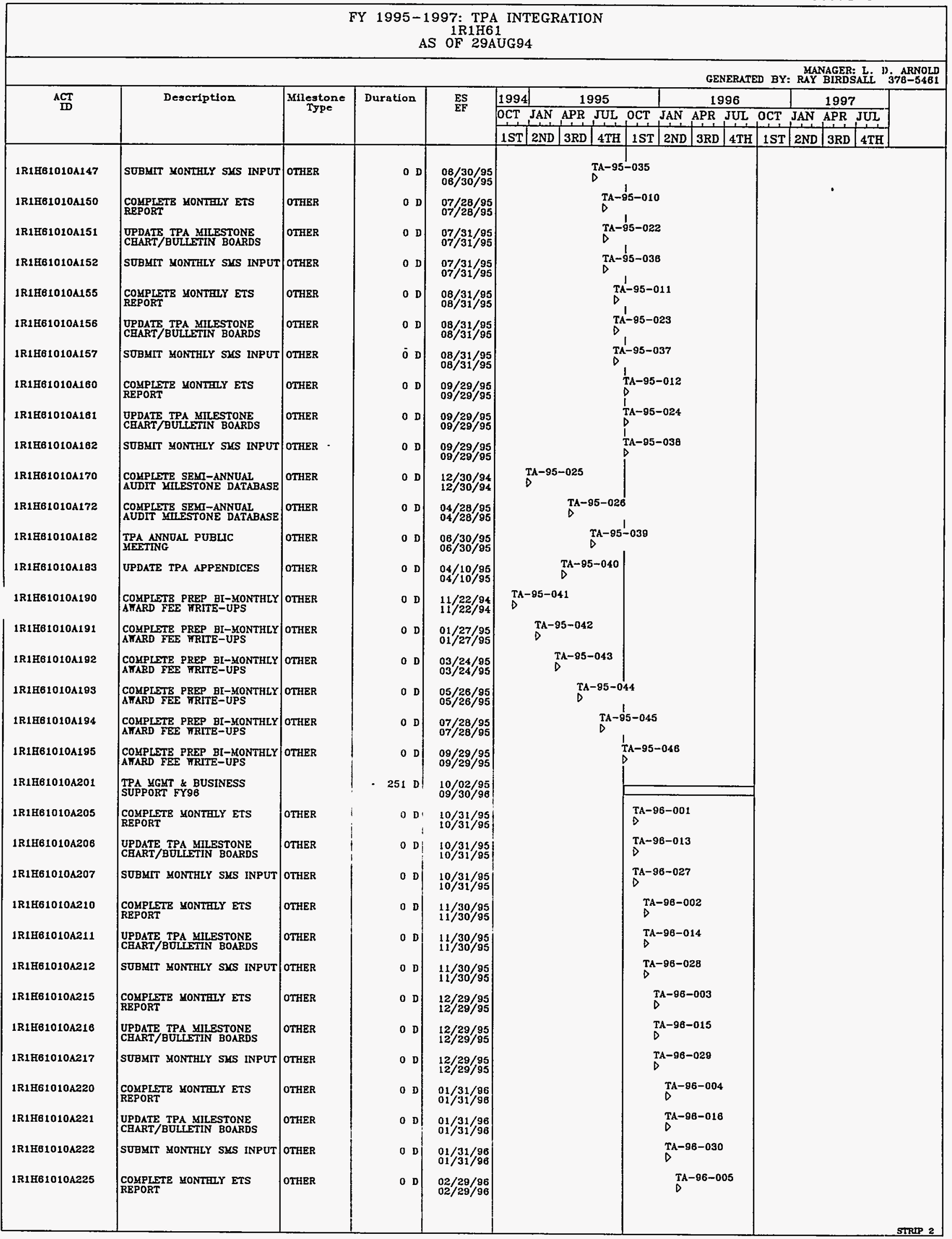




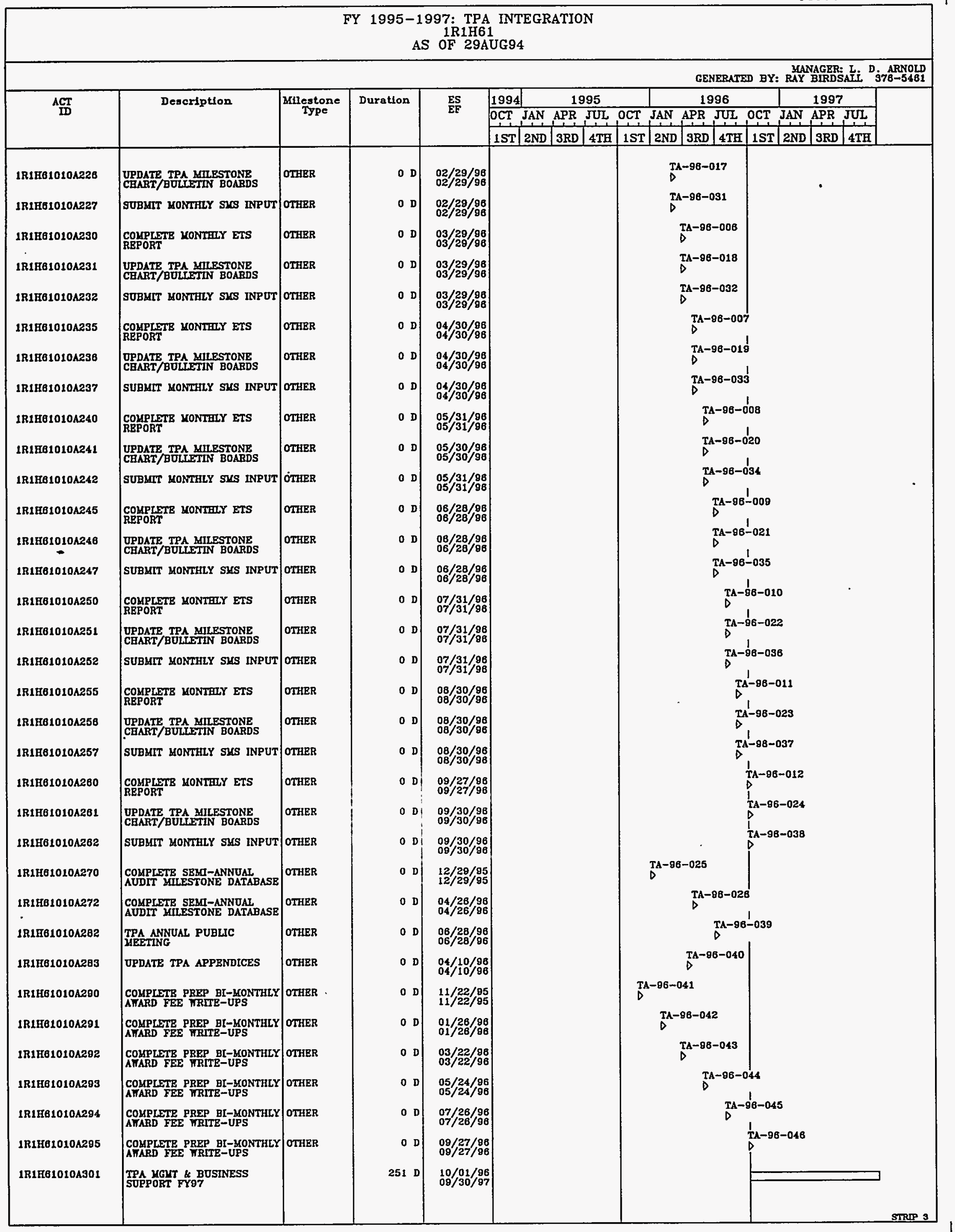




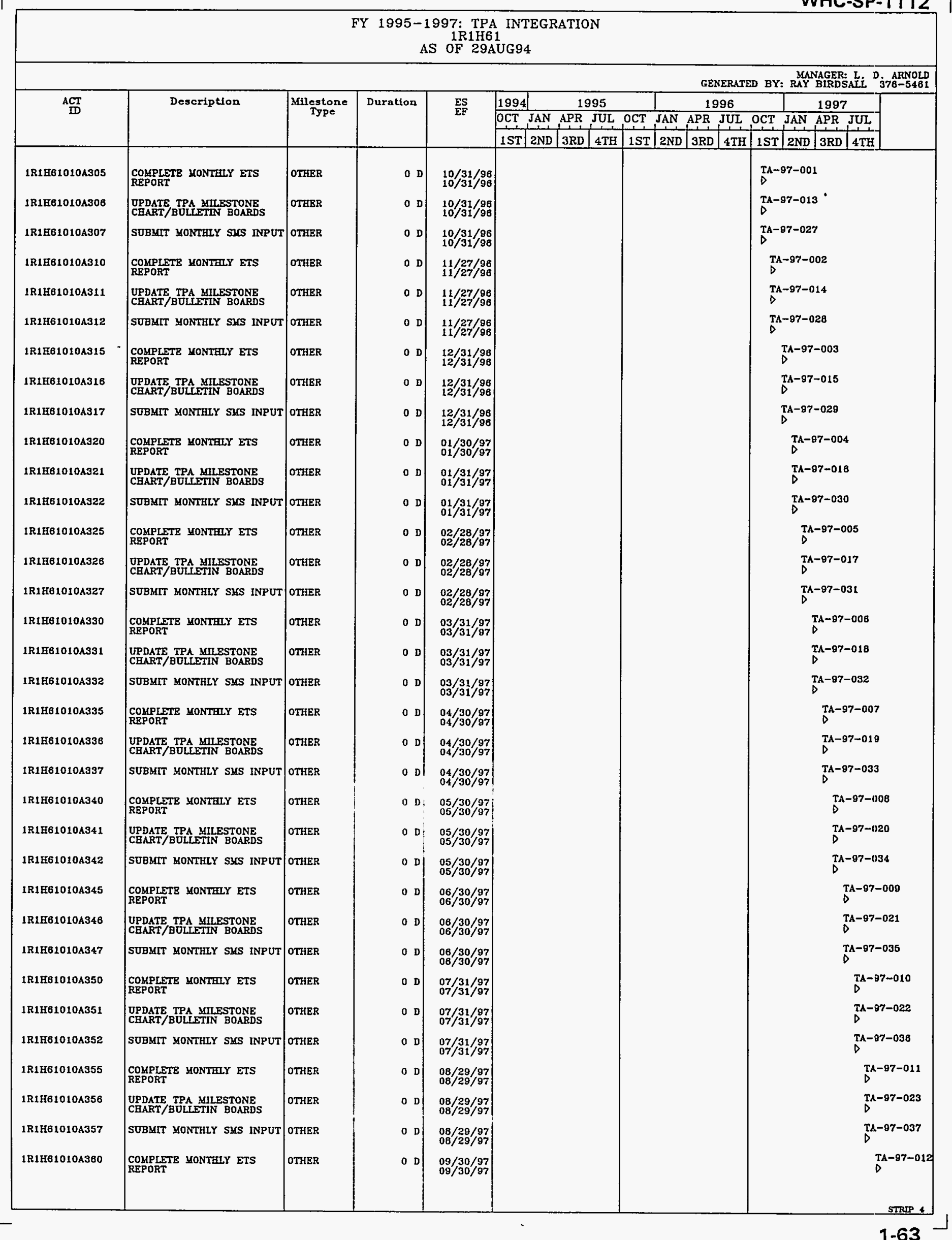


FY 1995-1997: TPA INTEGRATION

1R1H61

AS OF 29AUG94

GENERATED BY: RAYY BIRDSAIII

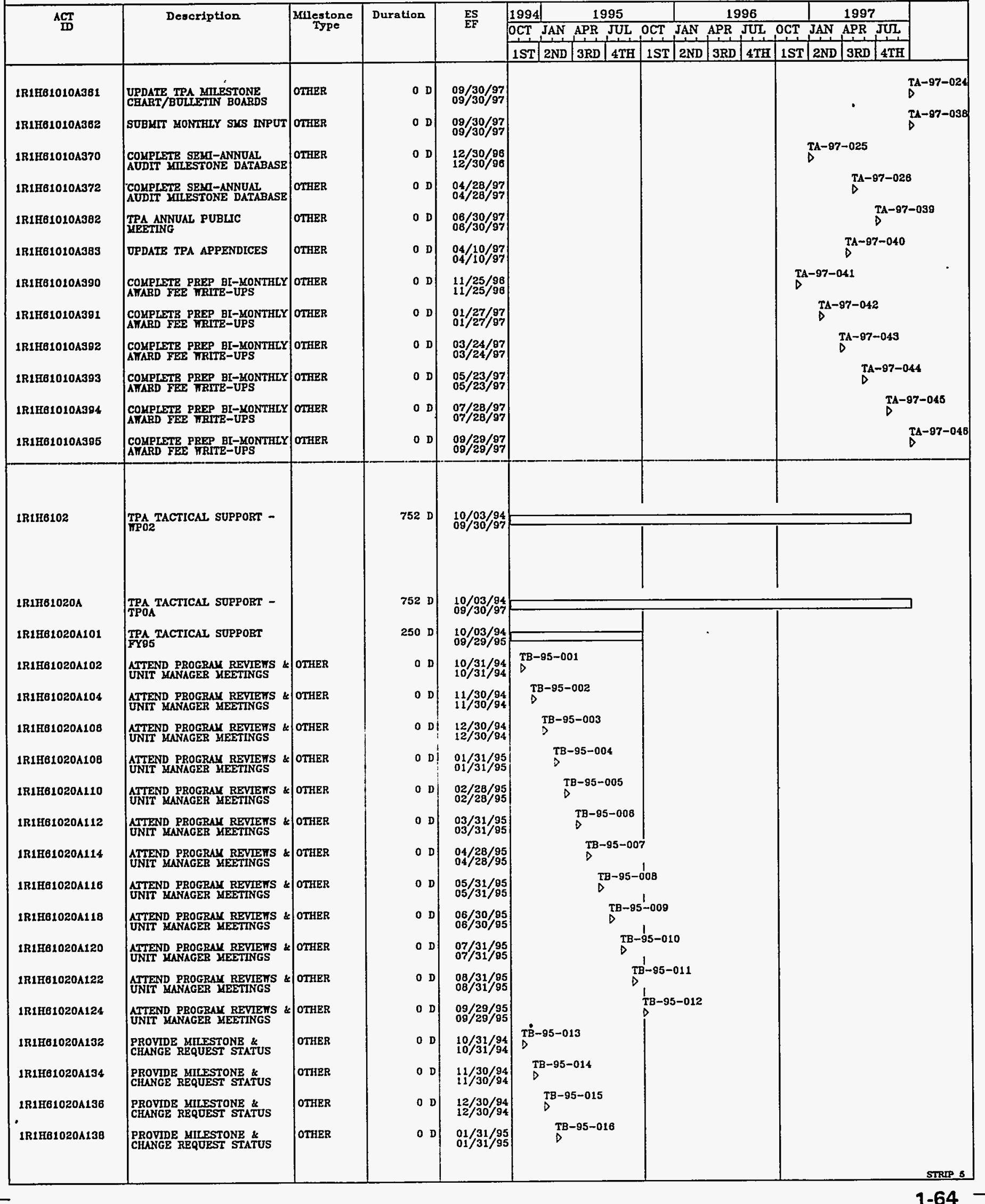


FY 1995-1997: TPA INTEGRATION

$1 \mathrm{R} 1 \mathrm{H} 61$

AS OF 29AUG94

GENERATED BY: MAYAGER: $L$. D. ARNOLD

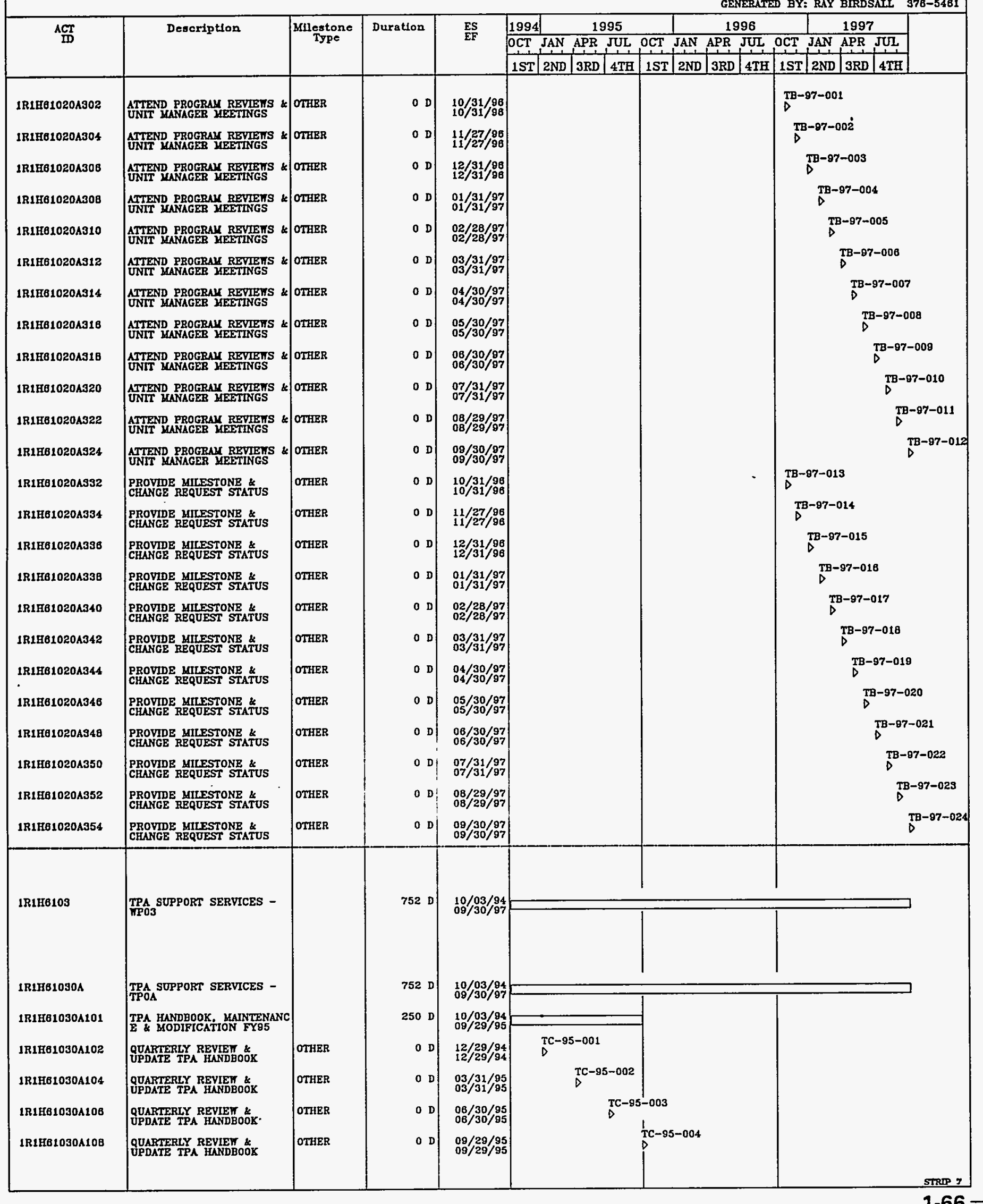


FY 1995-1997: TPA INTEGRATION

1R1H61

AS OF 29AUG94

GENERATED BY: RAY BIRDSALtí "376RNOLD

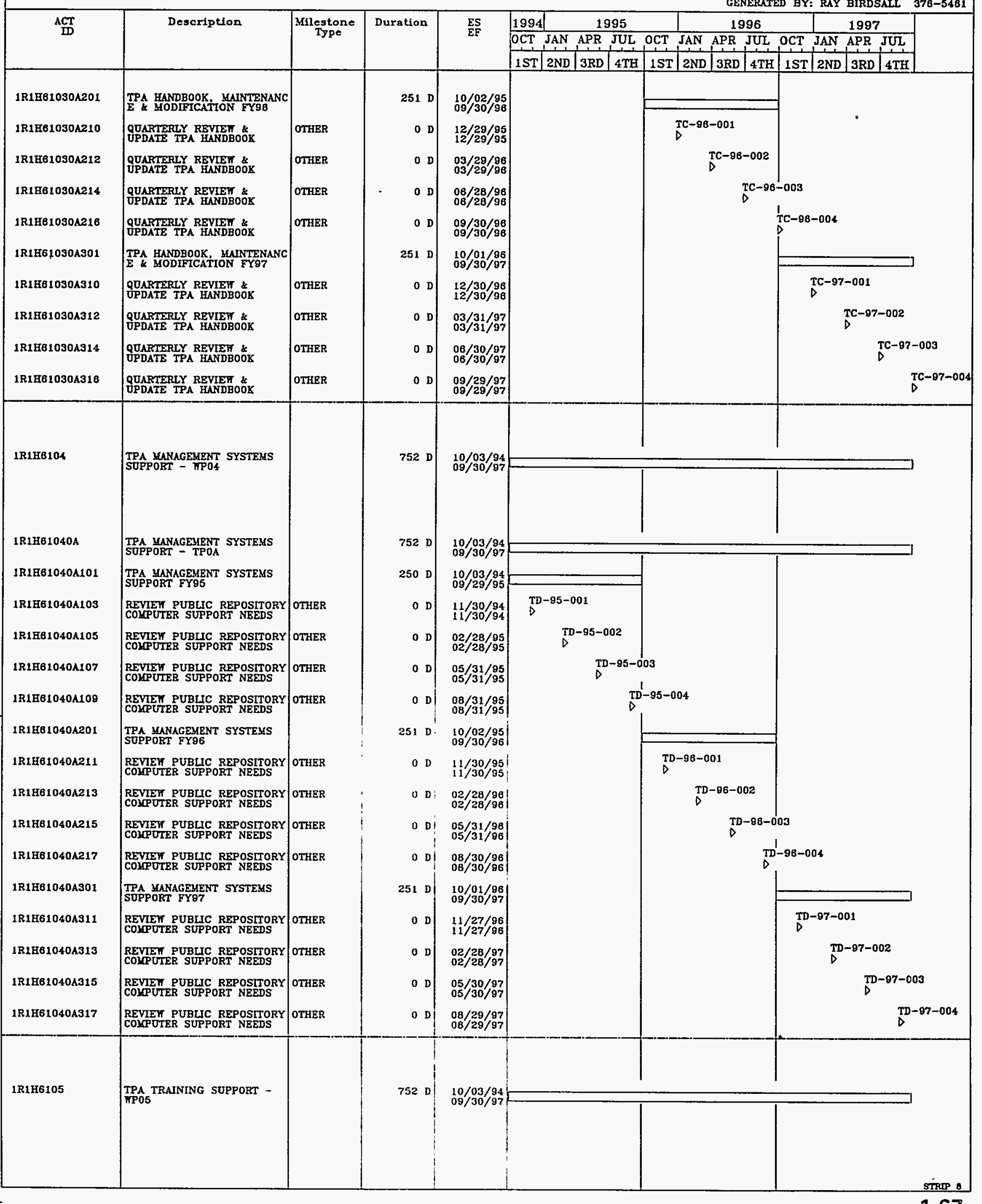


FY 1995-1997: TPA INTEGRATION

1R1H61

AS OF 29AUG94

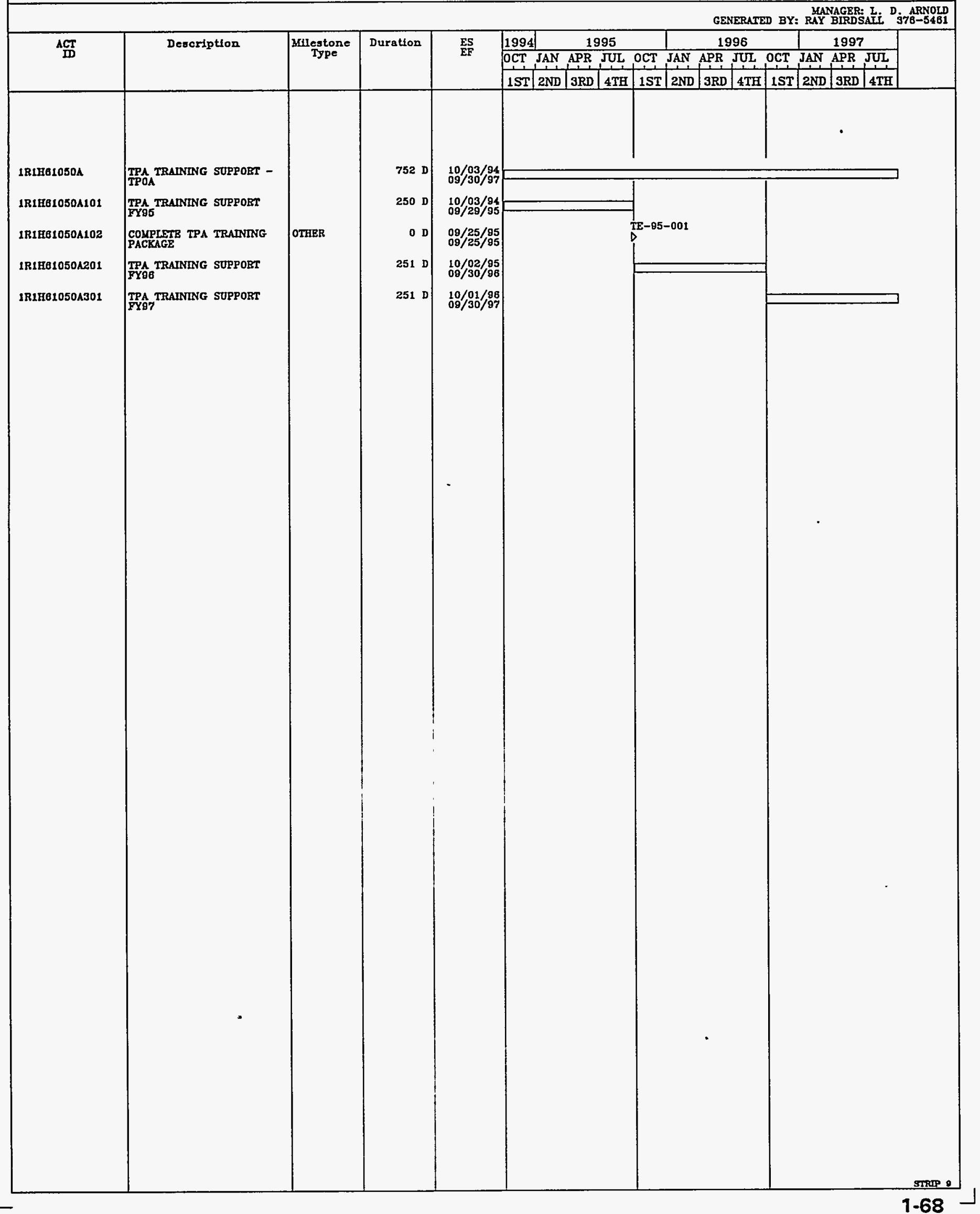




\section{FY 1995-1997: RCRA PERMIT COMPLIANCE/MODIFICATION $1 R 1 \mathrm{H} 71$ AS OF 29AUG94}

GENERATED BY: RAY BIRDSAIT ${ }_{378-54 B 1}$

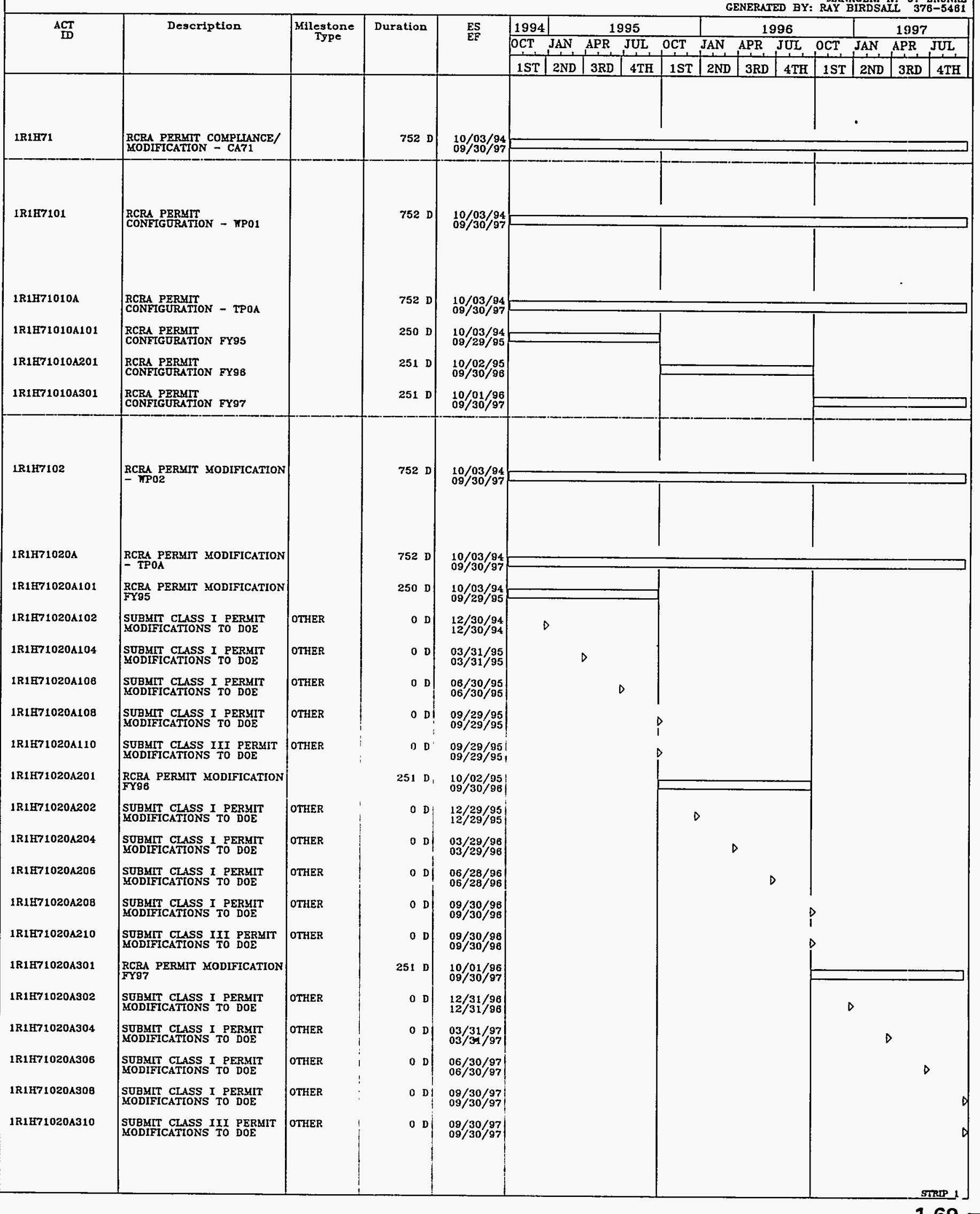


FY 1995-1997: RCRA PERMIT COMPLIANCE/MODIFICATION

$1 R 1 H 71$

AS OF 29AUG94

GENERTTED BY: RAY BATAGER: Ri "

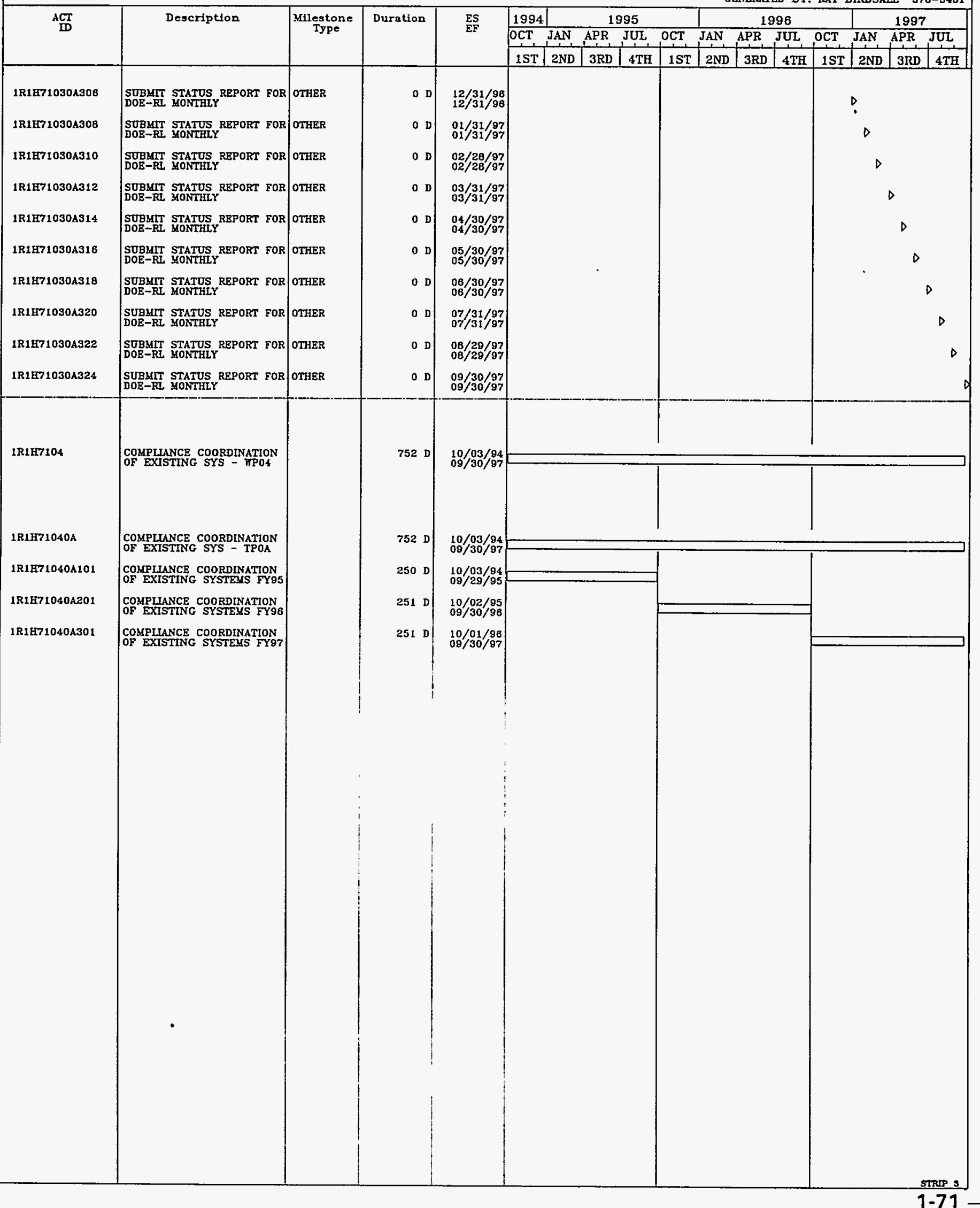


FY 1995-1997: RCRA PERMIT CONDITION TRACKING 1R1H72

AS OF 29AUG94

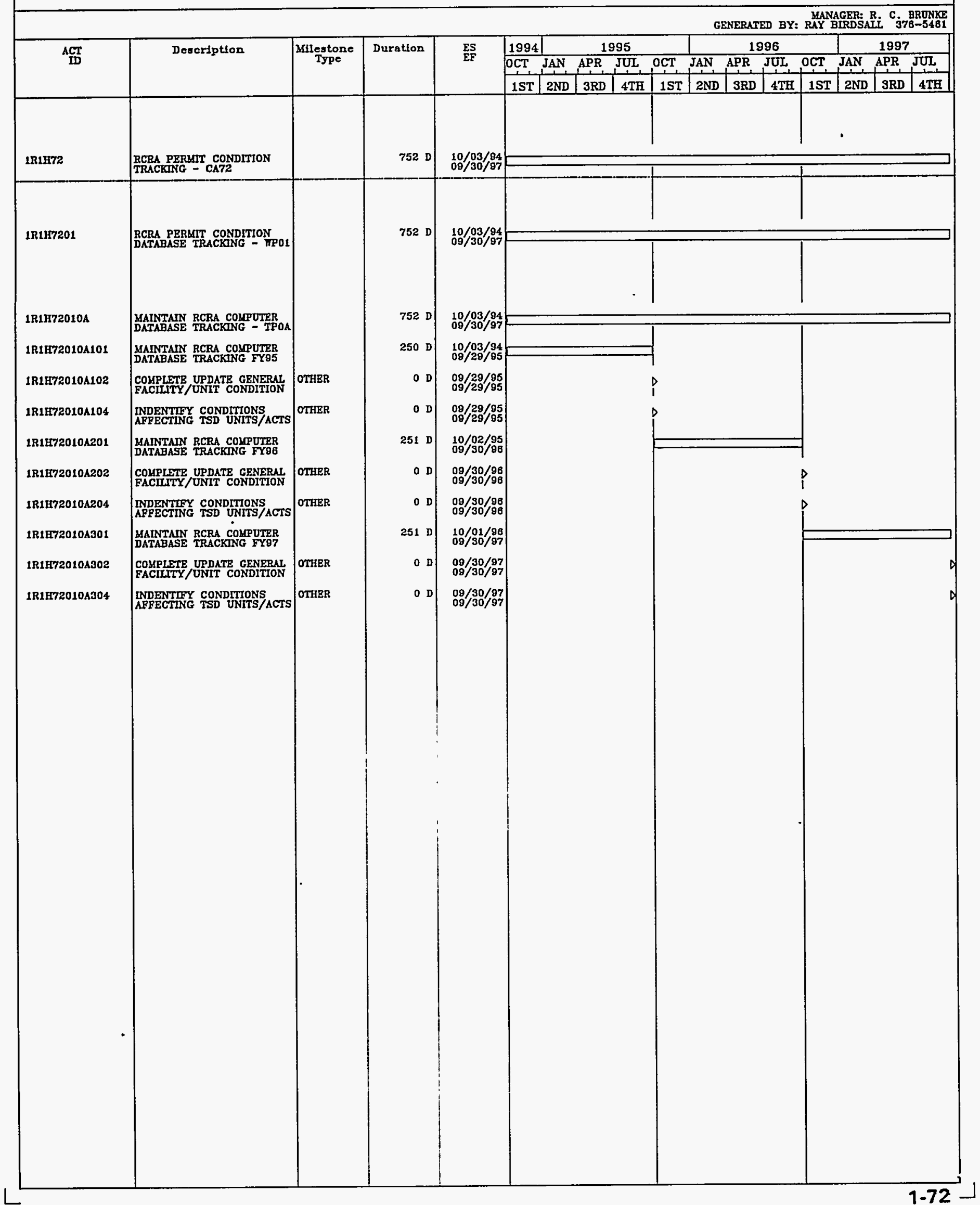


FY 1995-1997: RCRA PERMIT RECORD-KEEPING \& RETRIEVAL 1R1H73 AS OF 29AUG94

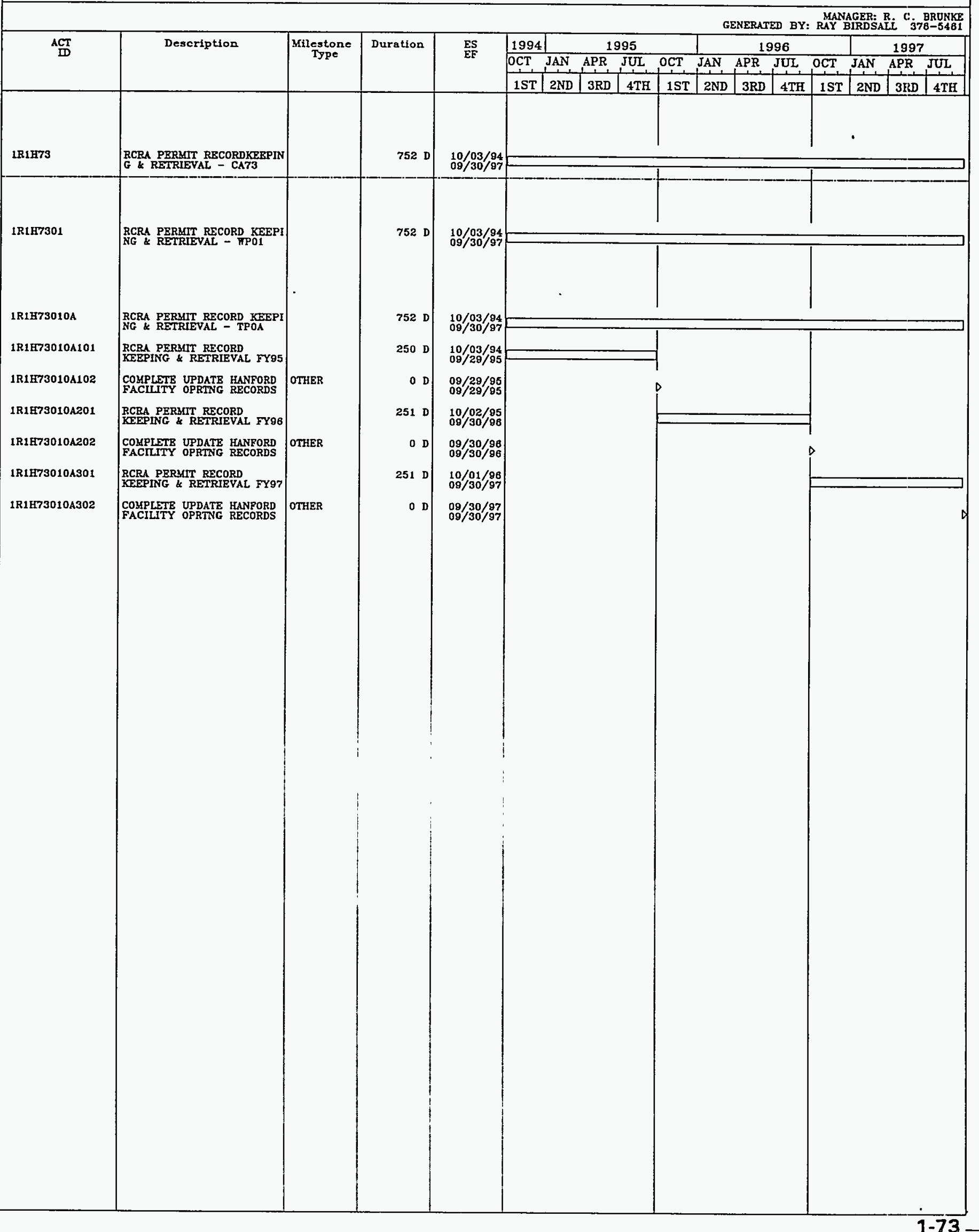


FY 1995-1997: ANN CLOSE COST EST \& WASTE MIN REPTS 1R1H75

AS OF 29AUG94

WHC-SP-1112

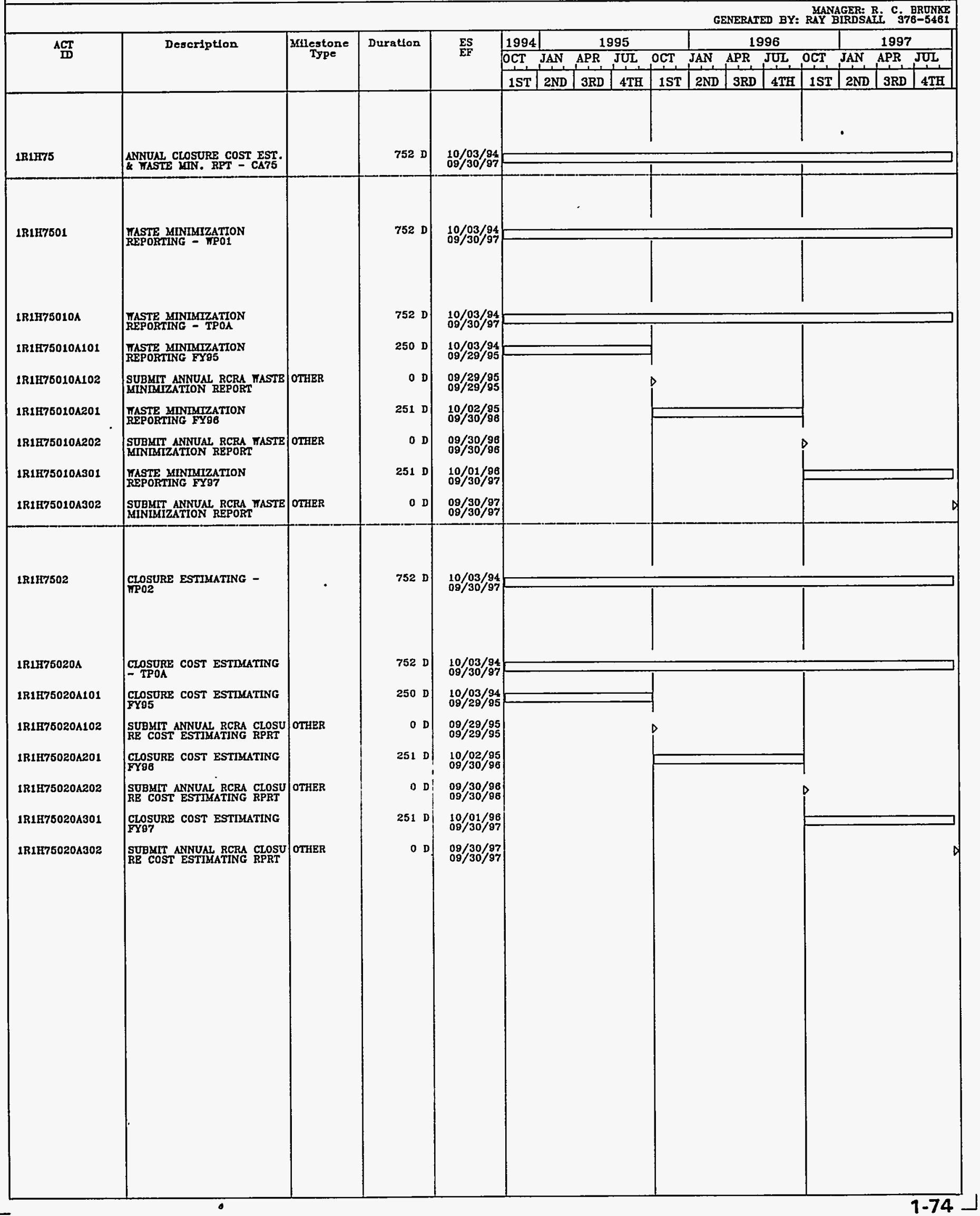


FY 1995-1997: RCRA PERMITS WASTE INVENTORY

$1 \mathrm{R} 1 \mathrm{H} 76$ AS OF 29AUG94

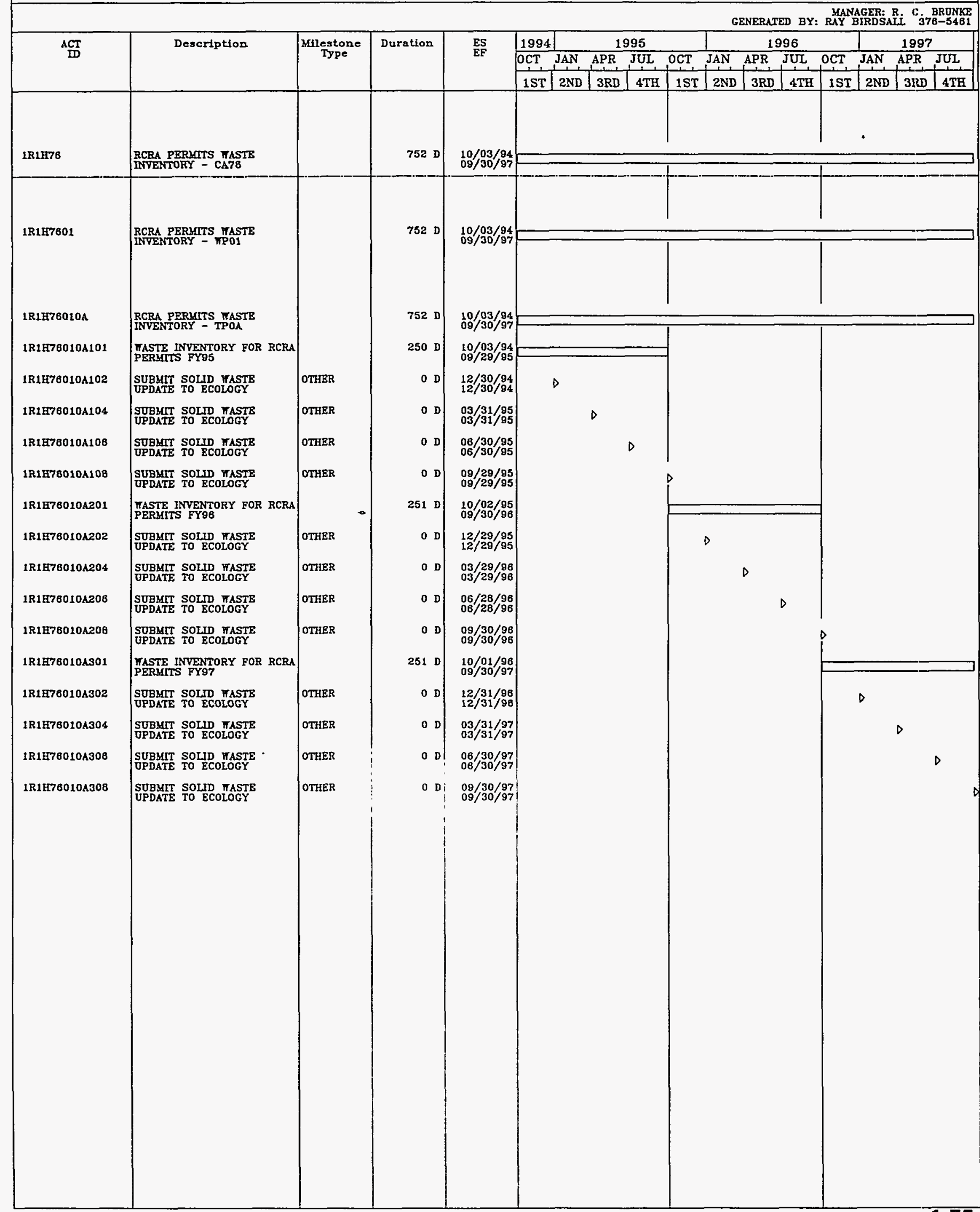




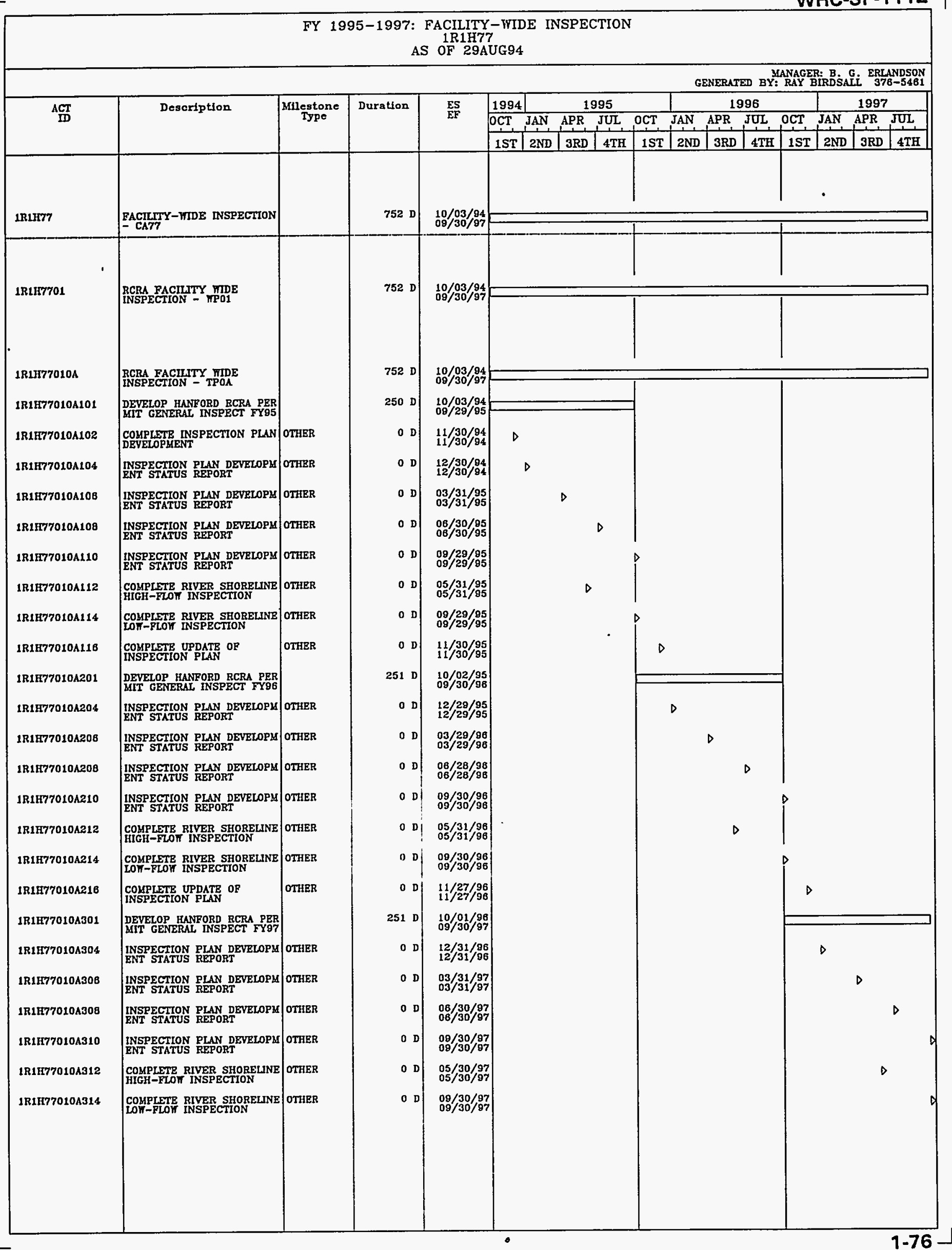


FY 1995-1997: RCRA PERMITTING COORDINATION $1 \mathrm{R} 1 \mathrm{H} 78$

AS OF 22SEP94

GENERATED BY: RAY MANAGER: S. H. PRDSALI PRICE

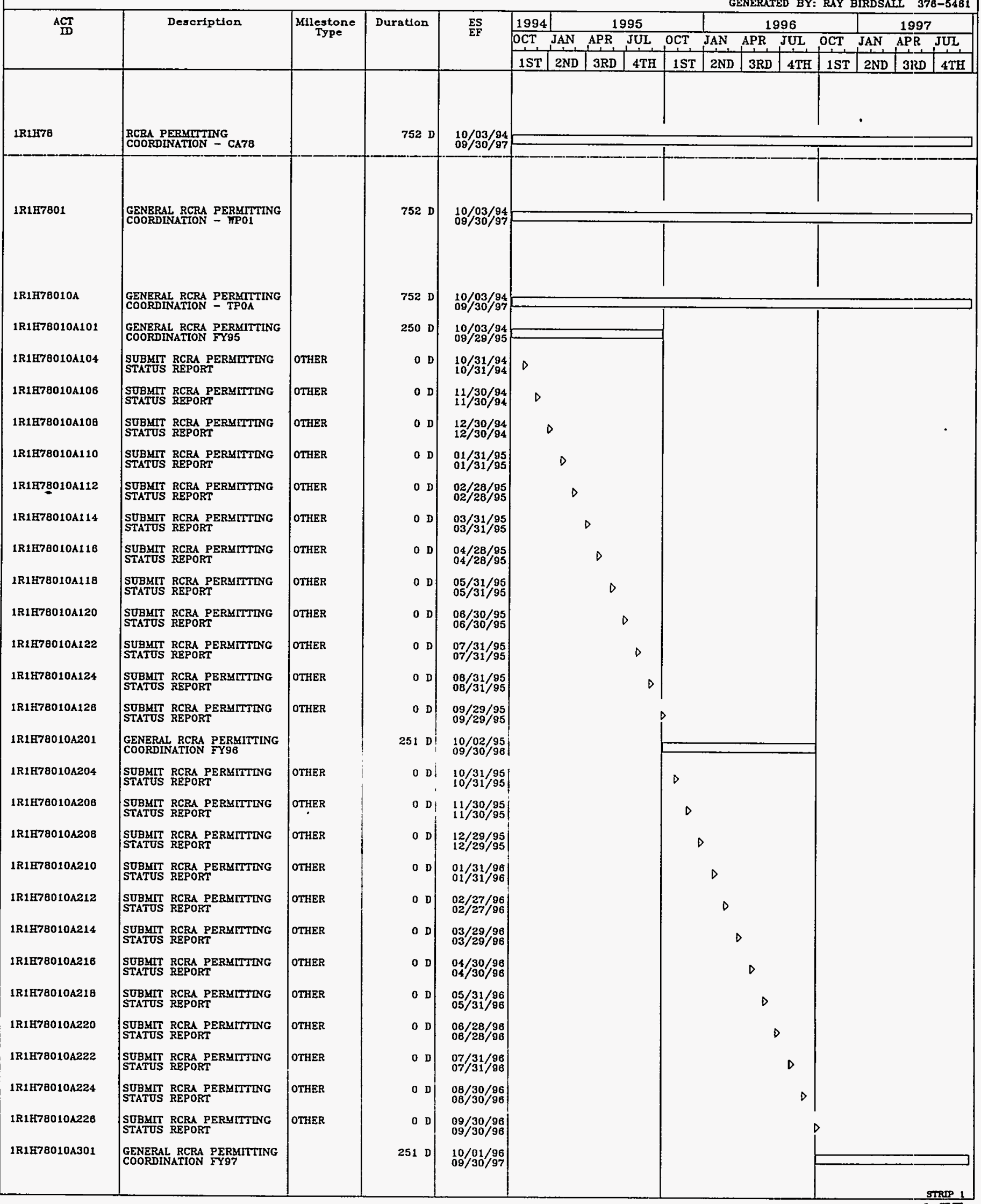


FY 1995-1997: RCRA PERMITTING COORDINATION 1R1H78

AS OF 22SEP94

GENERATED BY: RAY BANAGER:S. Y. Y. PRICE

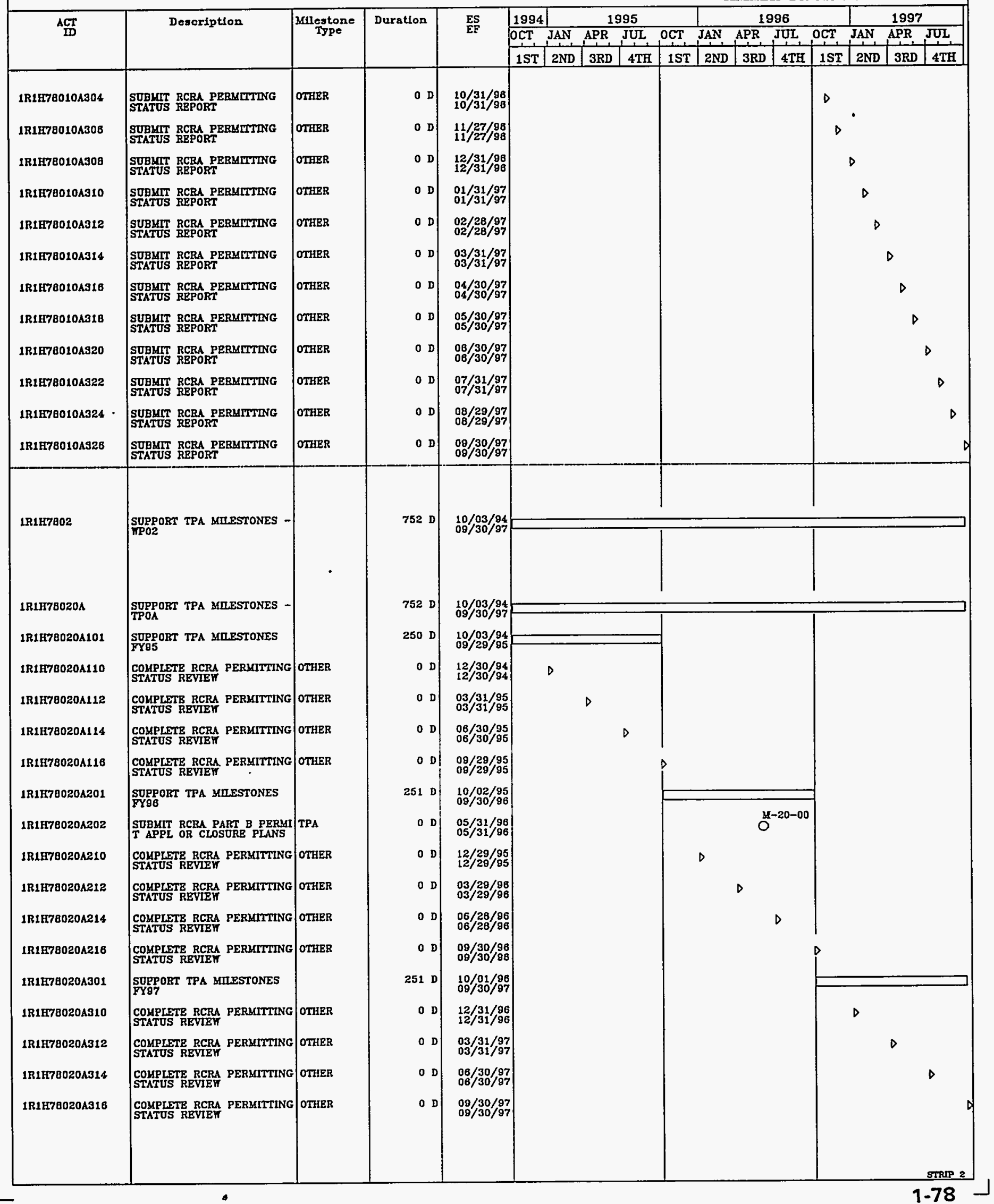


FY 1995-1997: POLLUTION PREVENTION 1R1H81

AS OF 22SEP94

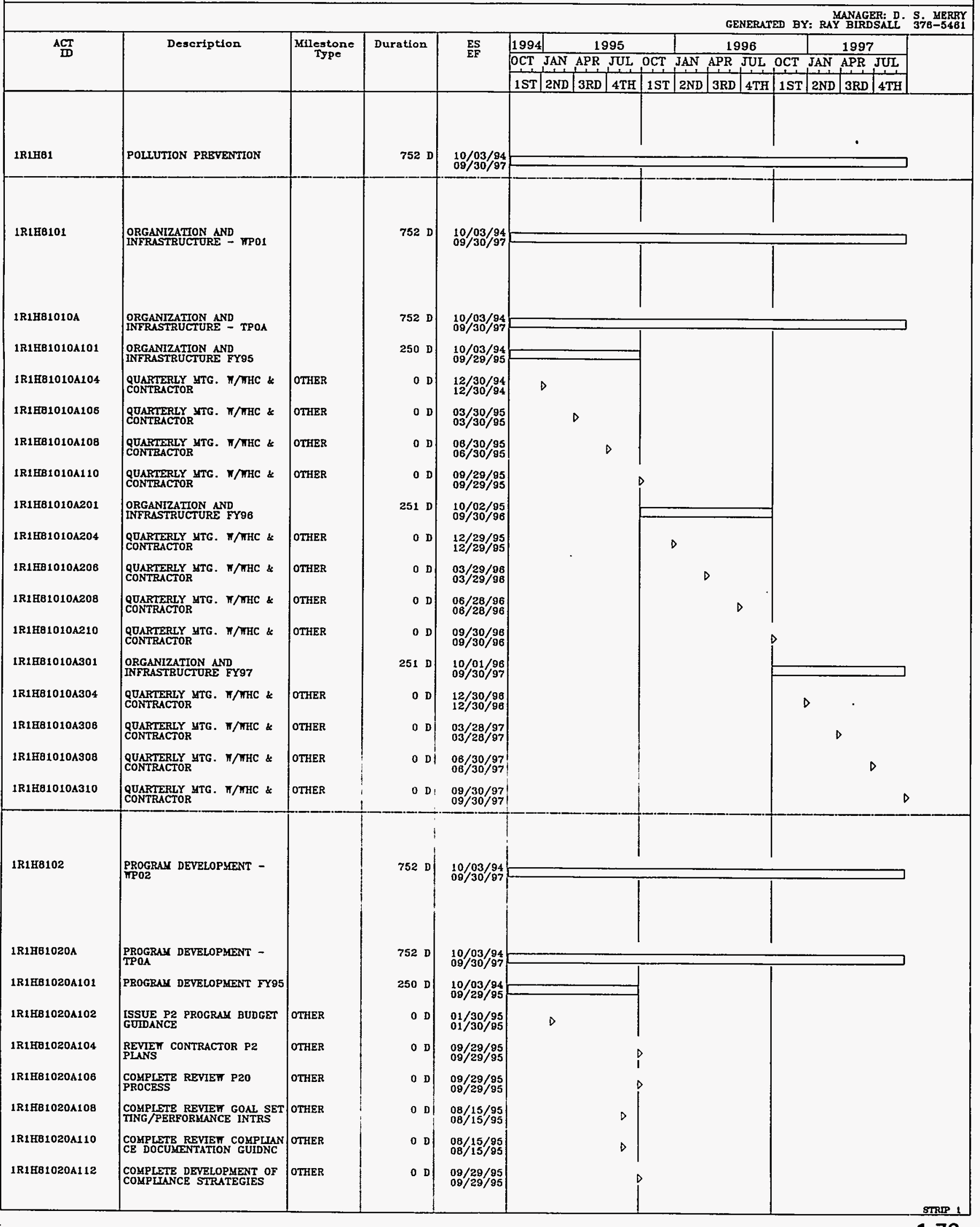




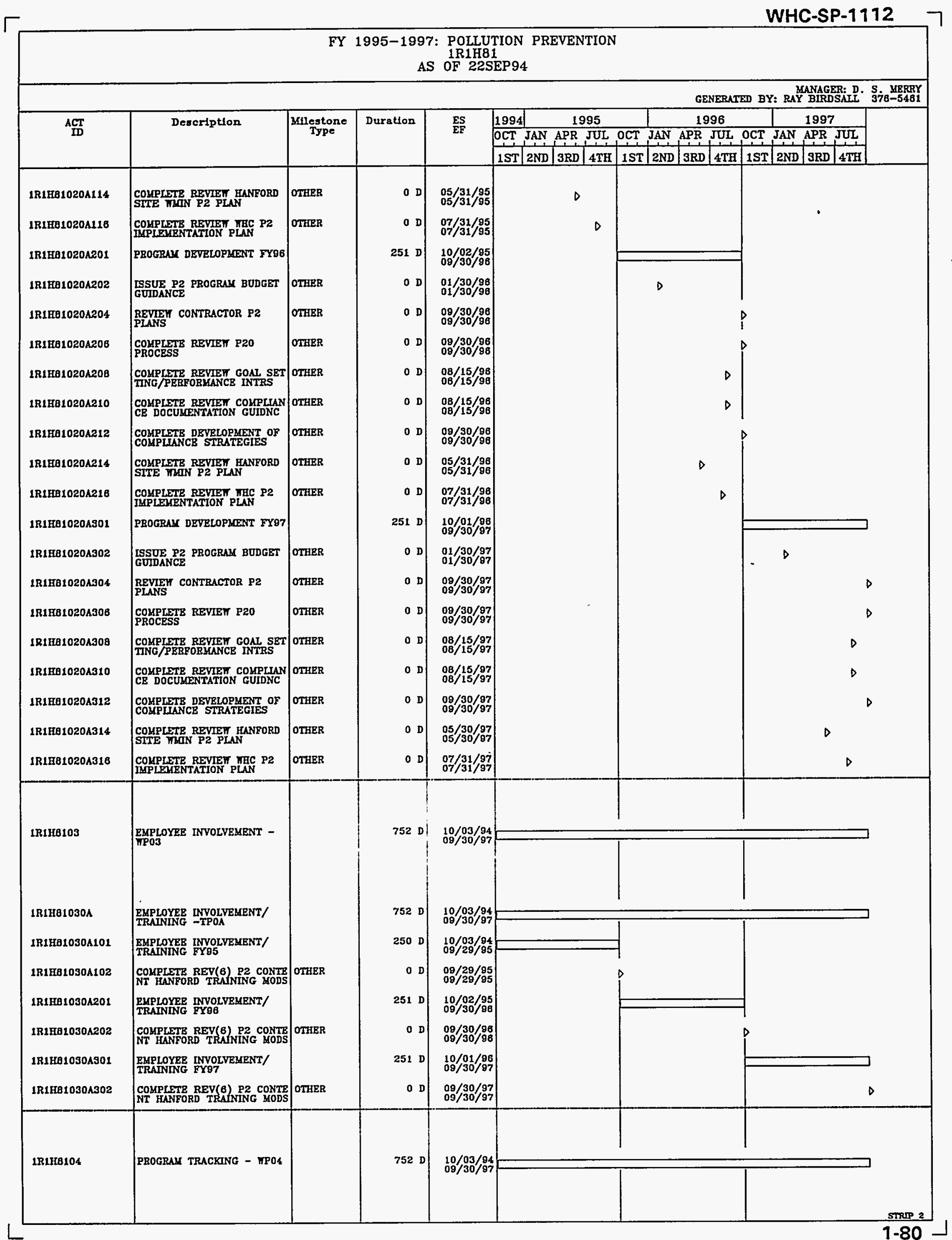


1 R1Ha

AS OF 22SEP94

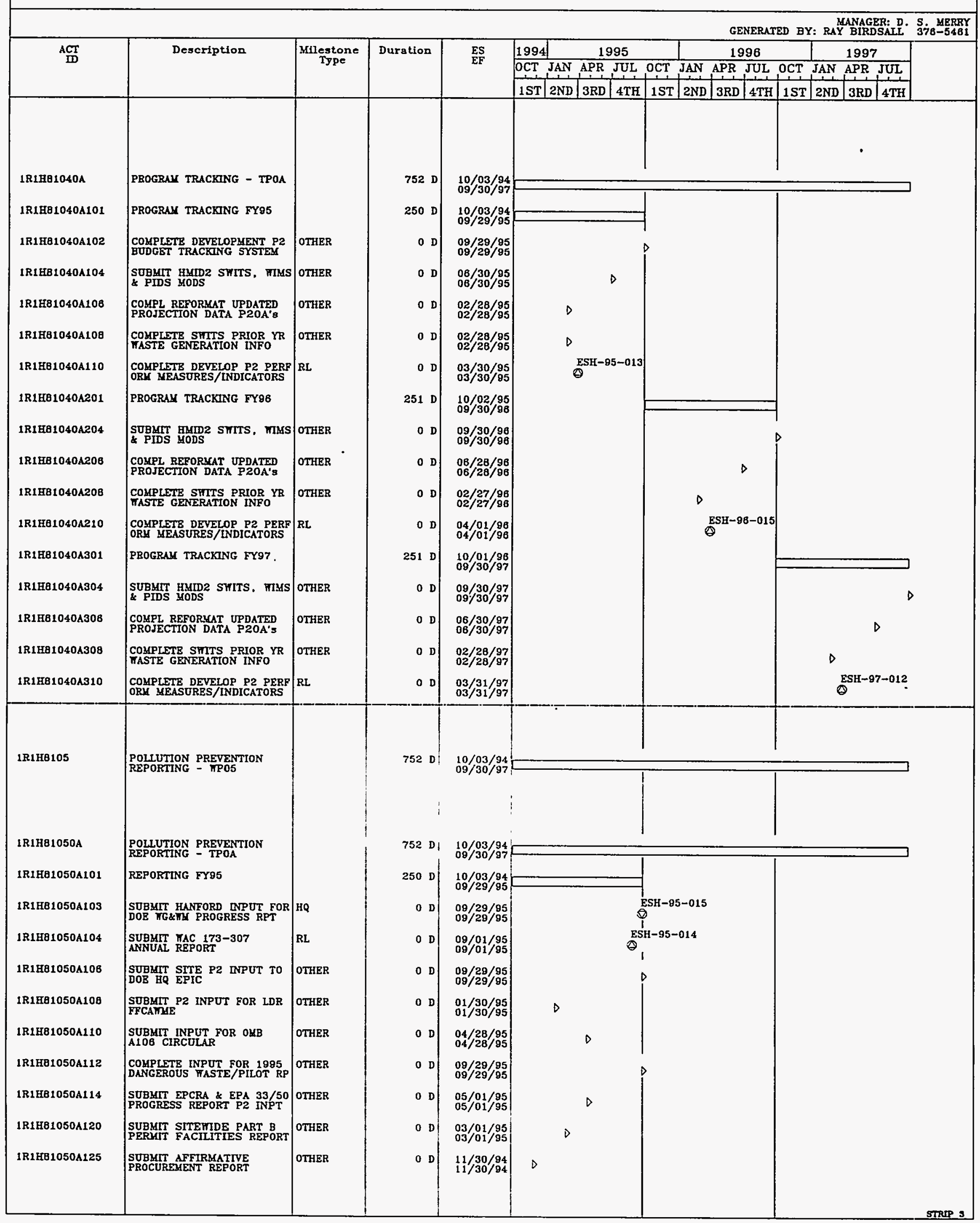




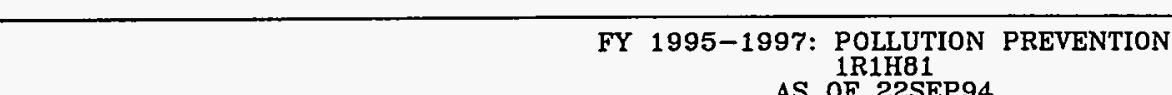

AS OF 22SEP94

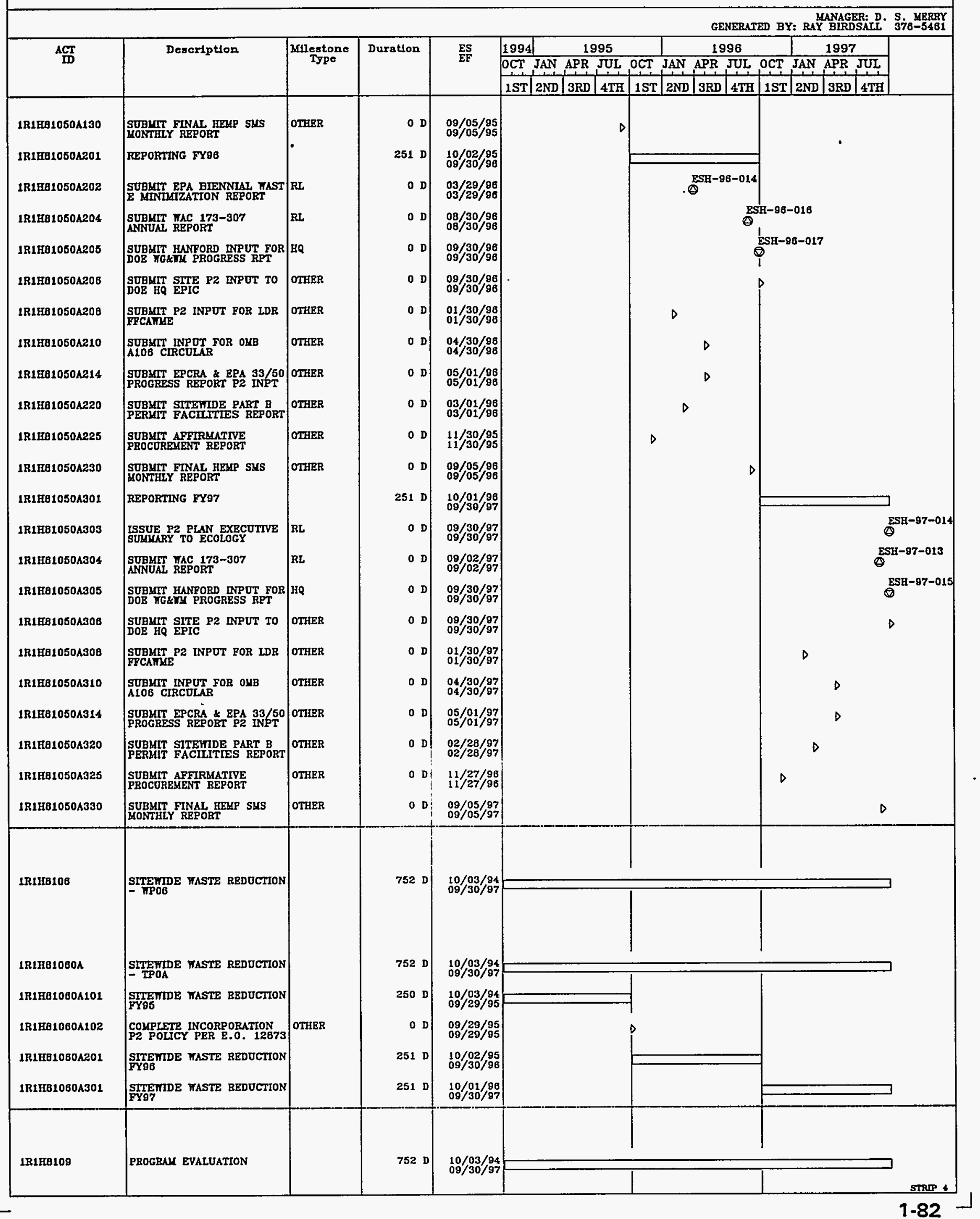




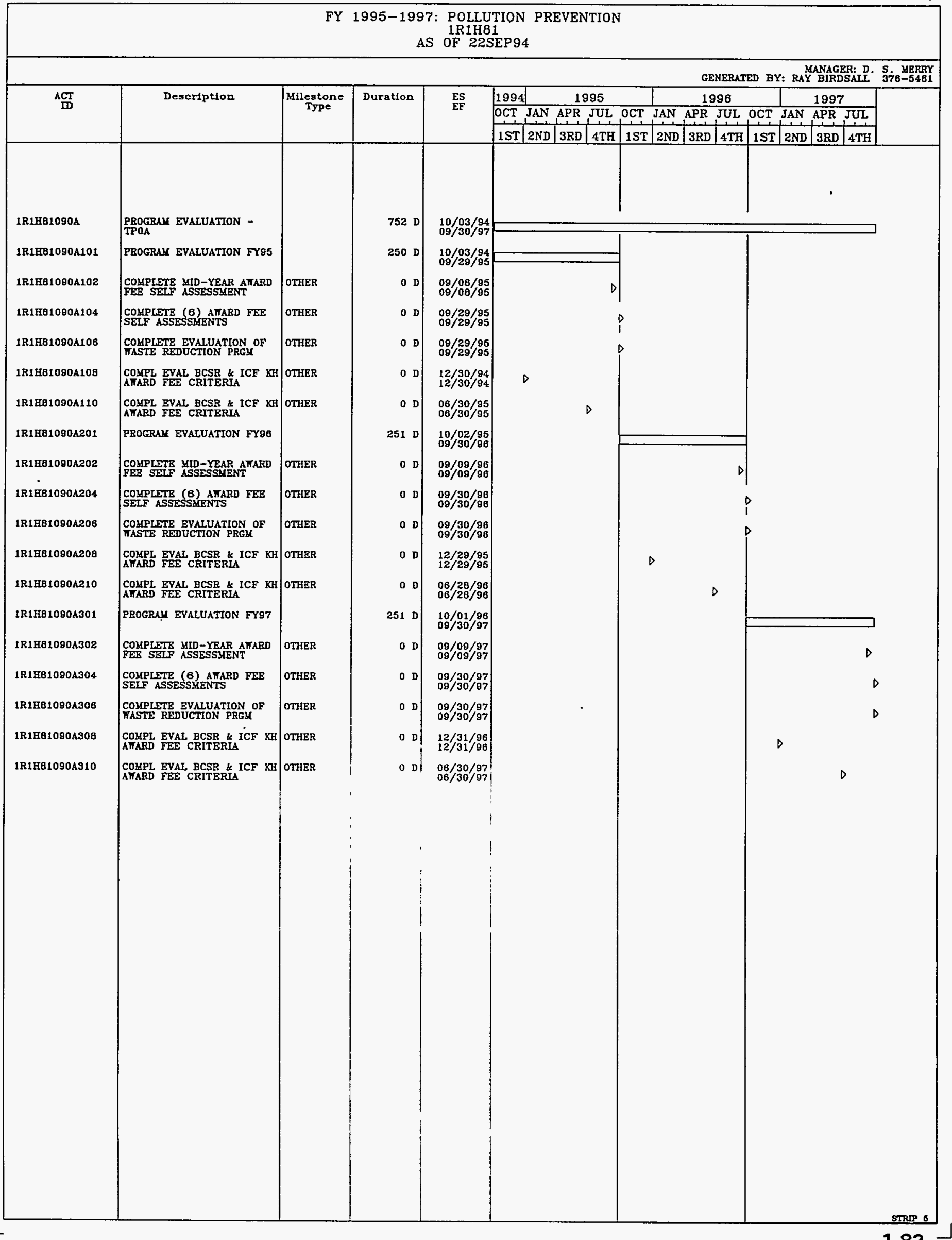


WBS 1.5.2.1

\begin{tabular}{|c|c|c|c|c|c|}
\hline $\begin{array}{l}\text { Milestone } \\
\text { Type* }\end{array}$ & $\begin{array}{l}\text { Control } \\
\text { Number }\end{array}$ & RL WBS & $\begin{array}{l}\text { ADS } \\
\text { NUMBER }\end{array}$ & Milestone Description & $\begin{array}{c}\text { Milestone } \\
\text { Completion } \\
\text { Date }\end{array}$ \\
\hline RL & ESH-95-001 & 1.5.2.1.5.1 & 7330 & EPCRA 311 MSDS List Quarterly Update & 11/01/94 \\
\hline RL & ESH-95-002 & 1.5.2.1.5.1 & 7330 & EPCRA 311 MSDS List Quarterly Update & $02 / 01 / 95$ \\
\hline RL & ESH-95-003 & 1.5.2.1.5.1 & 7330 & $\begin{array}{l}\text { Submit EPCRA } 312 \text { Tier Two Hazardous Chemical } \\
\text { Inventory Report }\end{array}$ & 03/01/95 \\
\hline RL & ESH-95-004 & 1.5.2.1.5.1 & 7330 & $\begin{array}{l}\text { Submit } 1994 \text { Hanford Site Annual Dangerous } \\
\text { Waste Report }\end{array}$ & 03/01/95 \\
\hline RL & ESH-95-005 & 1.5.2.1.5.1 & 7330 & EPCRA 311 MSDS List Quarterly Update & 05/01/95 \\
\hline RL & ESH-95-006 & 1.5.2.1.5.1 & 7330 & Submit EPCRA 313 Toxic Release Inventory Report & $06 / 30 / 95$ \\
\hline RL & ESH-95-007 & 1.5.2.1.5.1 & 7330 & $\begin{array}{l}\text { Submit } 1994 \text { Hanford Site Annual Polychlorinated } \\
\text { Biphenyl (PCB) Document Log }\end{array}$ & $06 / 30 / 95$ \\
\hline $\mathbf{R L}$ & ESH-95-008 & 1.5.2.1.5.1 & 7330 & $\begin{array}{l}\text { Submit Annual Polycholorinated Biphenyl (PCB) } \\
\text { Report }\end{array}$ & $07 / 14 / 95$ \\
\hline HQ & ESH-95-009 & 1.5.2.1.5.1 & 7330 & Complete Laboratory Activity Toxic Use Report & $08 / 01 / 95$ \\
\hline RL & ESH-95-010 & 1.5.2.1.5.1 & 7330 & EPCRA 311 MSDS List Quarterly Update & $08 / 01 / 95$ \\
\hline RL & ESH-95-011 & 1.5.2.1.5.1 & 7330 & Prepare Submittal to the National IDB & $08 / 31 / 95$ \\
\hline RL & ESH-95-012 & 1.5.2.1.5.3 & 7330 & Issue Annual LDR Status Report (M-26-01E) & $04 / 28 / 95$ \\
\hline RL & ESH-95-013 & 1.5.2.2.1.1 & 7332 & $\begin{array}{l}\text { Develop Three Performance Measures/Indicators for } \\
\text { P2 Progress }\end{array}$ & 03/30/95 \\
\hline RL & ESH-95-014 & 1.5.2.2.1.1 & 7332 & $\begin{array}{l}\text { Prepare and Submit WAC 173-307 Annual Progress } \\
\text { Report }\end{array}$ & $09 / 01 / 95$ \\
\hline
\end{tabular}


WBS 1.5.2.1

\begin{tabular}{|c|c|c|c|c|c|}
\hline $\begin{array}{l}\text { Milestone } \\
\text { Type* }\end{array}$ & $\begin{array}{l}\text { Control } \\
\text { Number }\end{array}$ & RL WBS & $\begin{array}{l}\text { ADS } \\
\text { NUMBER }\end{array}$ & Milestone Description & $\begin{array}{l}\text { Milestone } \\
\text { Completion } \\
\text { Date }\end{array}$ \\
\hline $\mathrm{HQ}$ & ESH-95-015 & 1.5.2.2.1.1 & 7332 & $\begin{array}{l}\text { Prepare Hanford Input to DOE Annual Report on } \\
\text { Waste Generation and Waste Minimization Progress } \\
\text { (SEN-37-92) }\end{array}$ & 09/29/95 \\
\hline RL & ESH-96-001 & 1.5.2.1.5.1 & 7330 & EPCRA 311 MSDS List Quarterly Update & $11 / 01 / 95$ \\
\hline RL & ESH-96-002 & 1.5.2.1.5.1 & 7330 & Submit RCRA 3016 Report & $01 / 31 / 96$ \\
\hline RL & ESH-96-003 & 1.5.2.1.5.1 & 7330 & EPCRA 311 MSDS List Quarterly Update & $02 / 01 / 96$ \\
\hline RL & ESH-96-004 & 1.5.2.1.5.1 & 7330 & $\begin{array}{l}\text { Submit EPCRA } 312 \text { Tier Two Hazardous Chemical } \\
\text { Inventory Report }\end{array}$ & 03/01/96 \\
\hline RL & ESH-96-005 & 1.5.2.1.5.1 & 7330 & $\begin{array}{l}\text { Submit } 1995 \text { Hanford Site Annual Dangerous } \\
\text { Waste Report }\end{array}$ & $03 / 01 / 96$ \\
\hline $\mathbf{R L}$ & ESH-96-006 & 1.5.2.1.5.1 & 7330 & EPCRA 311 MSDS List Quarterly Update & $05 / 01 / 96$ \\
\hline $\mathbf{R L}$ & ESH-96-007 & 7.5.2.1.5.1 & 7330 & Submit EPCRA 313 Toxic Release Inventory Report & 07/01/96 \\
\hline RL & ESH-96-008 & 1.5.2.1.5.1 & 7330 & $\begin{array}{l}\text { Submit } 1995 \text { Hanford Site Annual Polychlorinated } \\
\text { Biphenyl (PCB) Document Log }\end{array}$ & 07/01/96 \\
\hline $\mathbf{R L}$ & ESH-96-009 & 1.5.2.1.5.1 & 7330 & $\begin{array}{l}\text { Submit Annual Polycholorinated Biphenyl (PCB) } \\
\text { Report }\end{array}$ & $07 / 15 / 96$ \\
\hline RL & ESH-96-010 & 1.5.2.1.5.1 & 7330 & EPCRA 311 MSDS List Quarterly Update & 08/01/96 \\
\hline RL & ESH-96-011 & 1.5.2.1.5.1 & 7330 & Prepare Submittal to the National IDB & 08/30/96 \\
\hline $\mathbf{R L}$ & ESH-96-012 & 1.5.2.1.5.3 & 7330 & Issue Annual LDR Status Report (M-26-01F) & $04 / 30 / 96$ \\
\hline RL & ESH-96-013 & 1.5.2.1.7.8 & 7330 & $\begin{array}{l}\text { Submit Part B Permit Applications or Closure Plans } \\
\text { for all RCRA (M-20-00) }\end{array}$ & $05 / 31 / 96$ \\
\hline
\end{tabular}




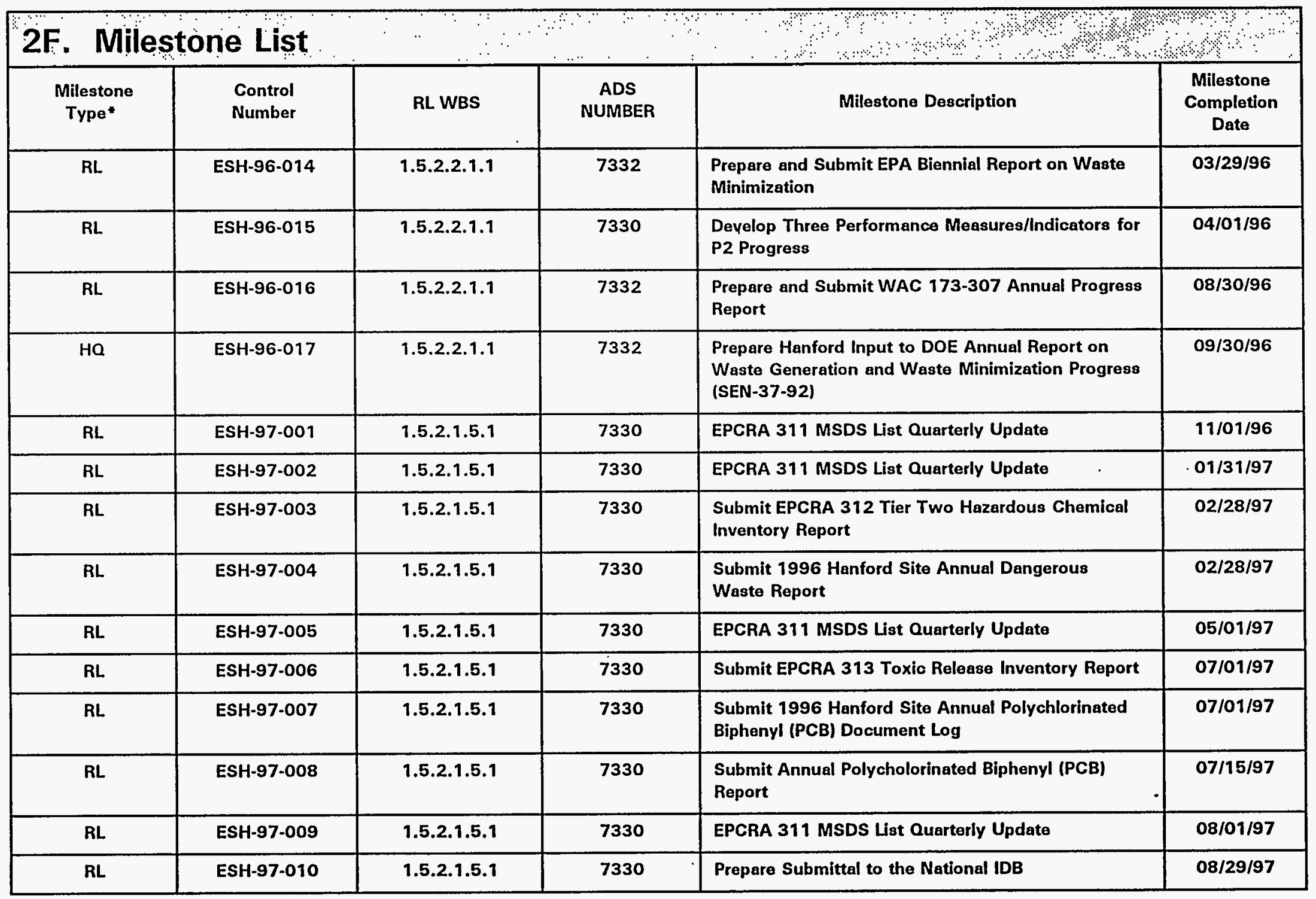




\section{WBS 1.5.2.1}

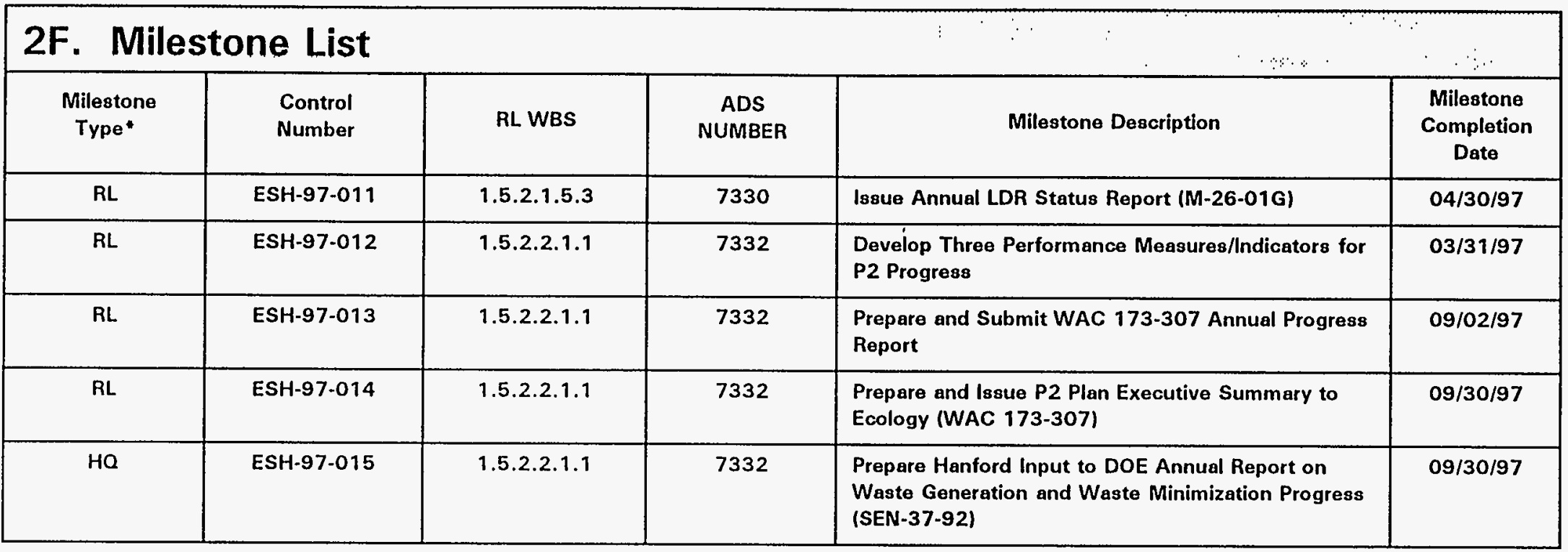

- TPA, HQ, RL, and selected contractor milestone

- On Tri-Party Agreement Milestones, also designate if they are HQ, RL 
HANFORD ENVIRONMENTAL MANAGEMENT PROGRAM

FY 1995 MYPP WBS 1.5.2.1

\section{G. Milestone Description Sheets}

See attached Milestone Description Sheets for FY 1995, 1996, and 1997. 
FY 1995 Milestone Description Sheets 


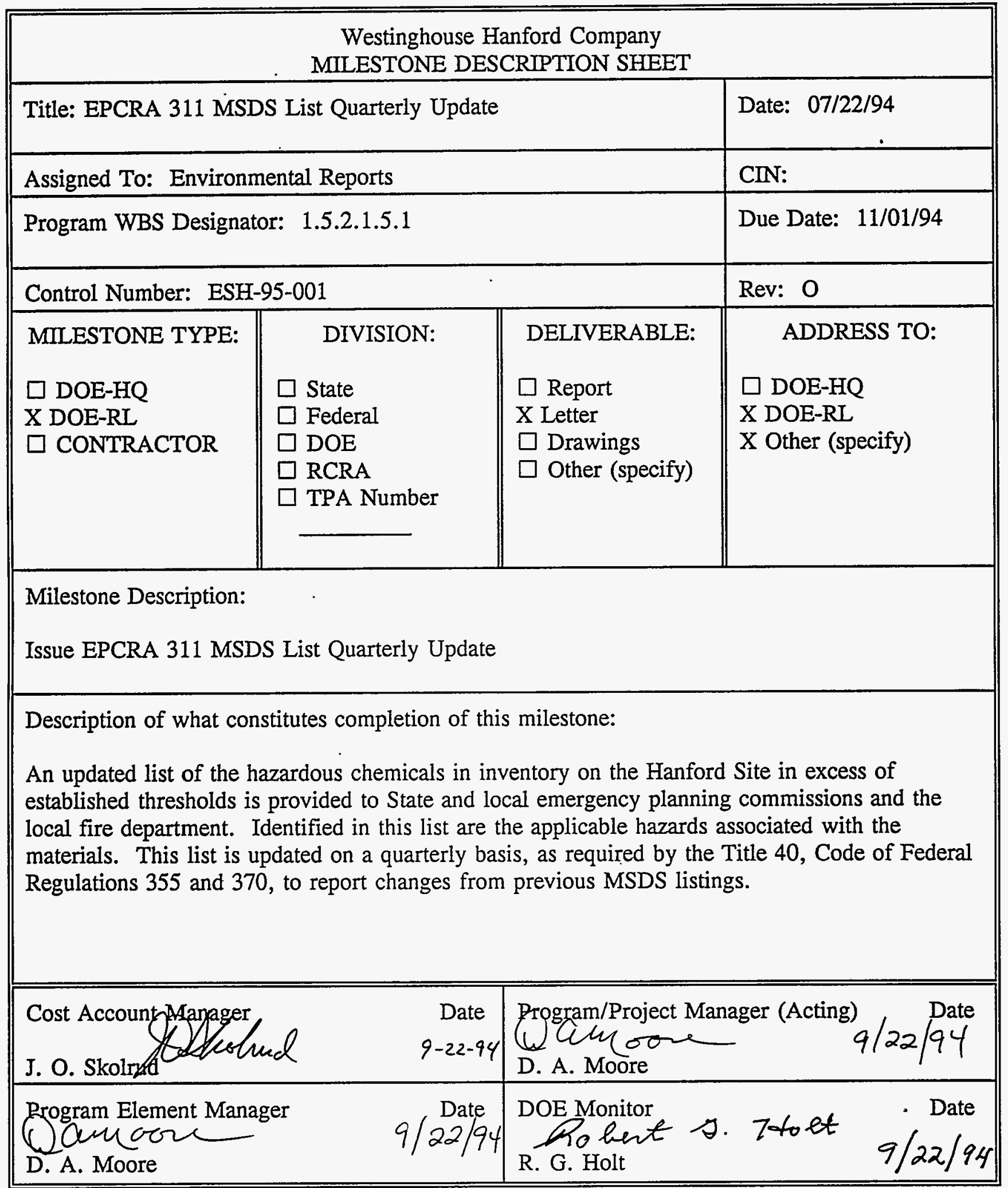




\begin{tabular}{||l|l||}
\hline \multicolumn{3}{|c|}{ Westinghouse Hanford Company } \\
MILESTONE DESCRIPTION SHEET
\end{tabular}




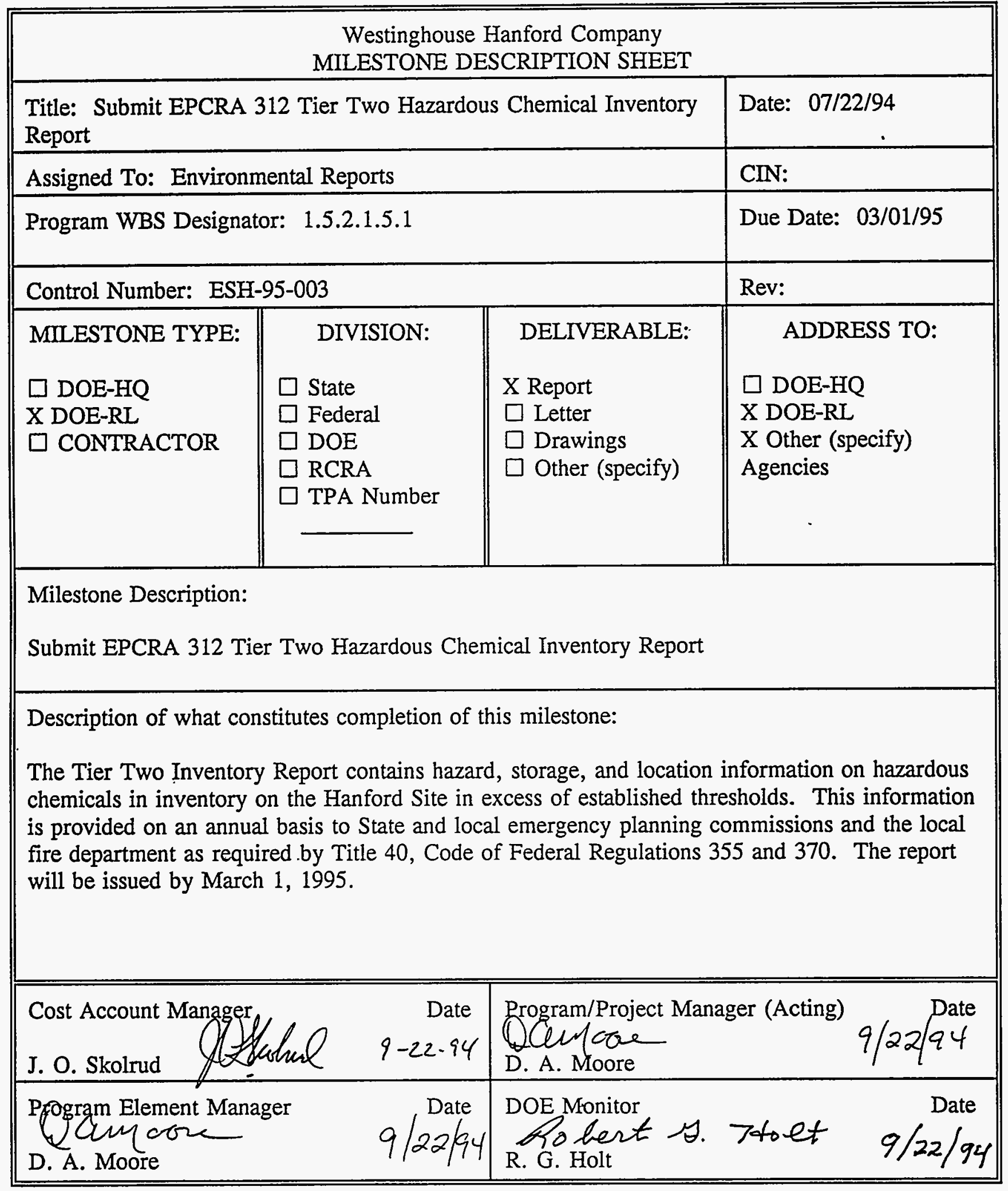




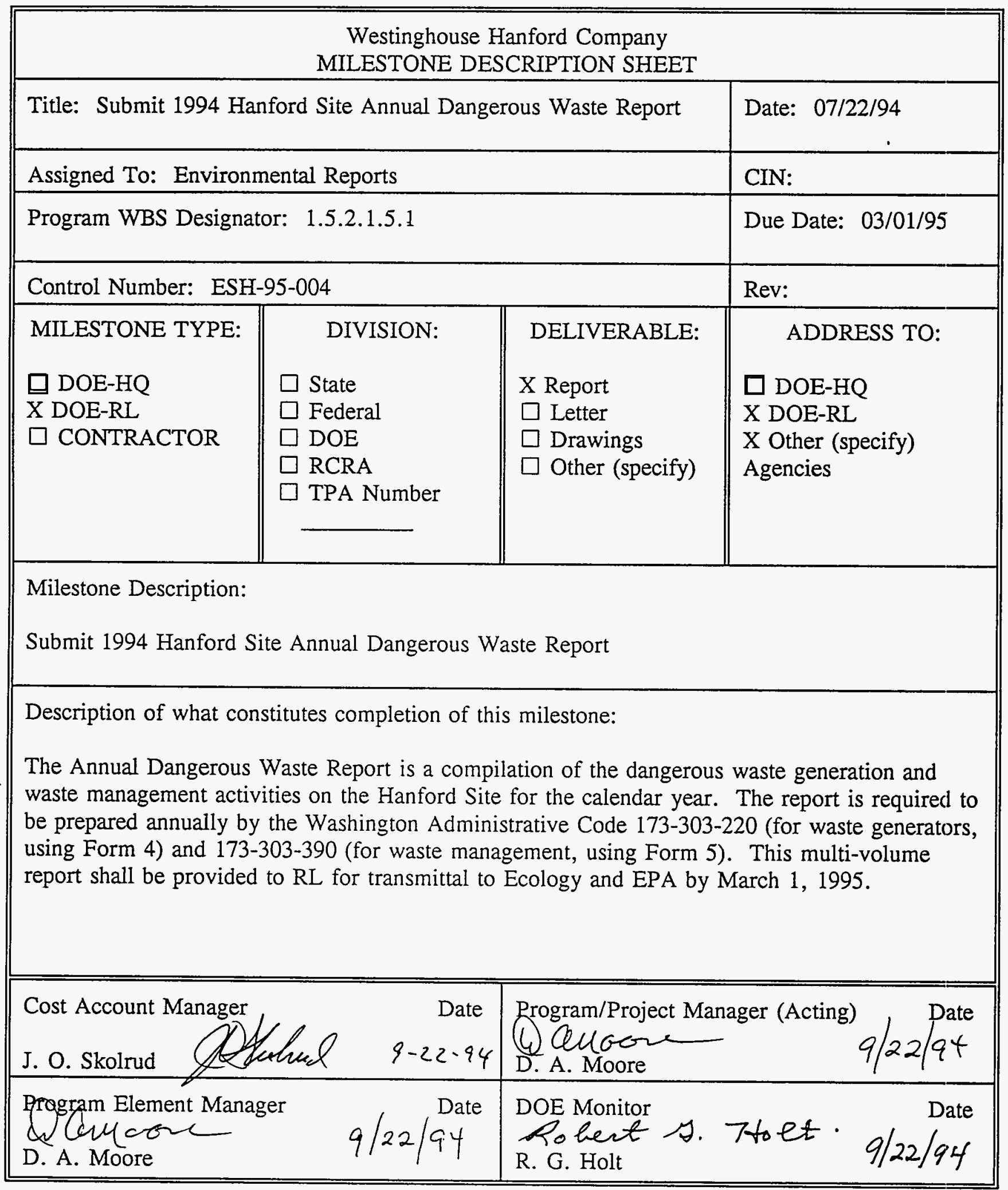




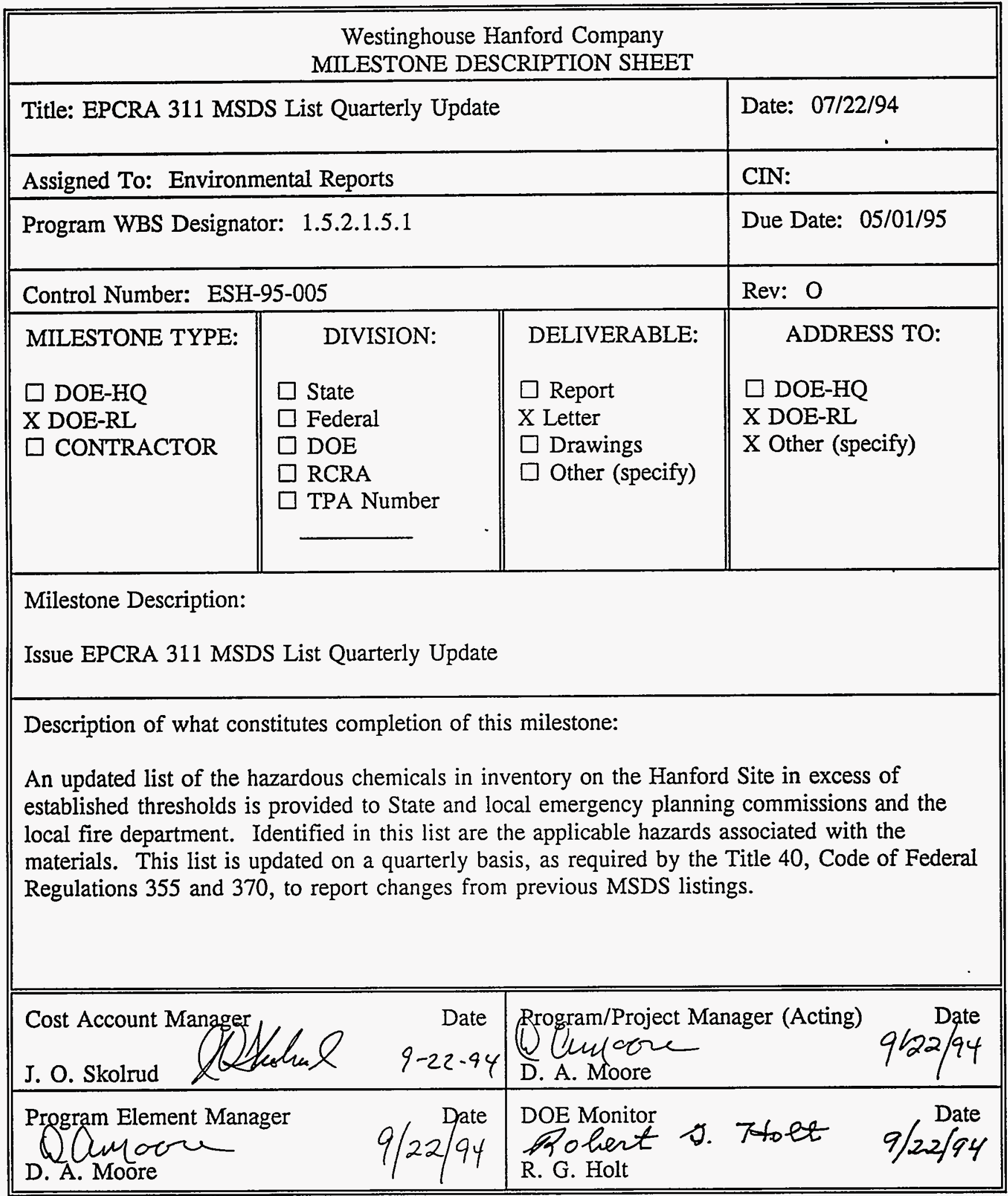




\begin{tabular}{|c|c|c|c|}
\hline \multicolumn{4}{|c|}{$\begin{array}{l}\text { Westinghouse Hanford Company } \\
\text { MILESTONE DESCRIPTION SHEET }\end{array}$} \\
\hline \multicolumn{3}{|c|}{ Title: Submit EPCRA 313 Toxic Release Inventory Report } & Date: $07 / 22 / 94$ \\
\hline \multicolumn{3}{|c|}{ Assigned To: Environmental Reports } & CIN: \\
\hline \multicolumn{3}{|c|}{ Program WBS Designator: $1.5 \cdot 2 \cdot 1.5 .1$} & Due Date: $06 / 30 / 95$ \\
\hline \multicolumn{3}{|c|}{ Control Number: ESH-95-006 } & Rev: \\
\hline MILESTONE TYPE: & DIVISION: & DELIVERABLE: & ADDRESS TO: \\
\hline $\begin{array}{l}\square \text { DOE-HQ } \\
\text { X DOE-RL } \\
\square \text { CONTRACTOR }\end{array}$ & $\begin{array}{l}\square \text { State } \\
\square \text { Federal } \\
\square \text { DOE } \\
\square \text { RCRA } \\
\square \text { TPA Number }\end{array}$ & $\begin{array}{l}\text { X Report } \\
\square \text { Letter } \\
\square \text { Drawings } \\
\square \text { Other (specify) }\end{array}$ & $\begin{array}{l}\square \text { DOE-HQ } \\
\text { X DOE-RL } \\
\text { X Other (specify) }\end{array}$ \\
\hline \multicolumn{4}{|c|}{$\begin{array}{l}\text { Milestone Description: } \\
\text { Submit EPCRA } 313 \text { Toxic Release Inventory Report }\end{array}$} \\
\hline \multicolumn{4}{|c|}{$\begin{array}{l}\text { Description of what constitutes completion of this milestone: } \\
\text { The Toxic Chemical Release Report provides information to the public regarding the use and } \\
\text { release of toxic chemicals in excess of specific threshold quantities and pollution prevention } \\
\text { activities associated with those chemicals. The requirements and format are identified in the } \\
\text { Title } 40 \text {, Code of Federal Regulations } 372 \text {. This report is submitted to the EPA and the State on } \\
\text { an annual basis and will be provided July 1, } 1995 \text {. }\end{array}$} \\
\hline \multicolumn{2}{|c|}{ Jost Account Manager } & \multicolumn{2}{|c|}{$\begin{array}{l}\text { Program/Project Manager (Acting) } \\
\text { (Cemoove } \\
\text { D. A. Moore }\end{array}$} \\
\hline \multicolumn{2}{|c|}{$\begin{array}{l}\text { Program Element Manager } \\
\text { D. A. Moncone }\end{array}$} & \multicolumn{2}{|c|}{$\begin{array}{l}\text { DOE Monitor } \\
\text { Po Pent } Q \text {. Jolt } 9 / 22 / 94 \\
\text { R. G. Holt }\end{array}$} \\
\hline
\end{tabular}




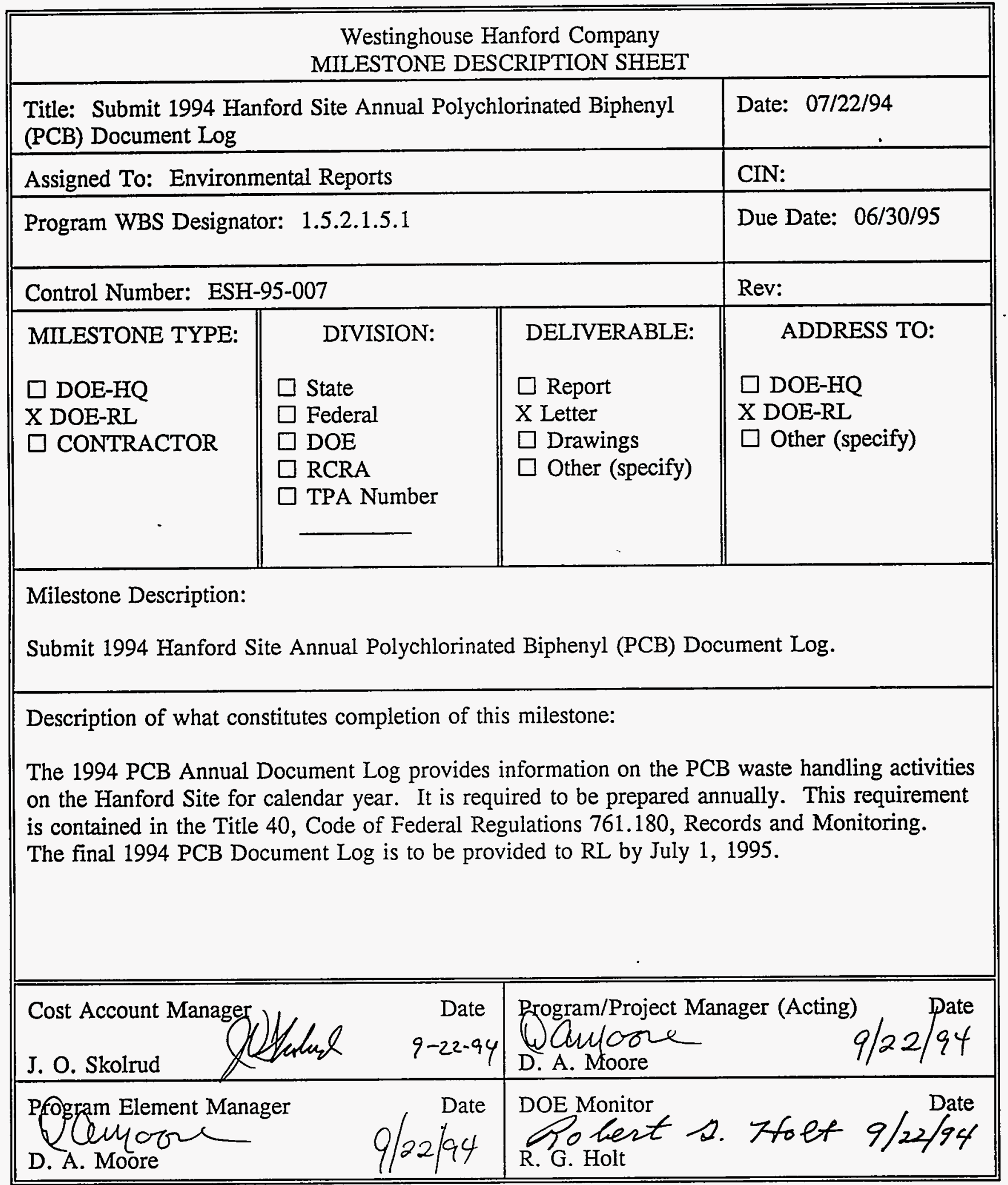


WHC-SP-1112

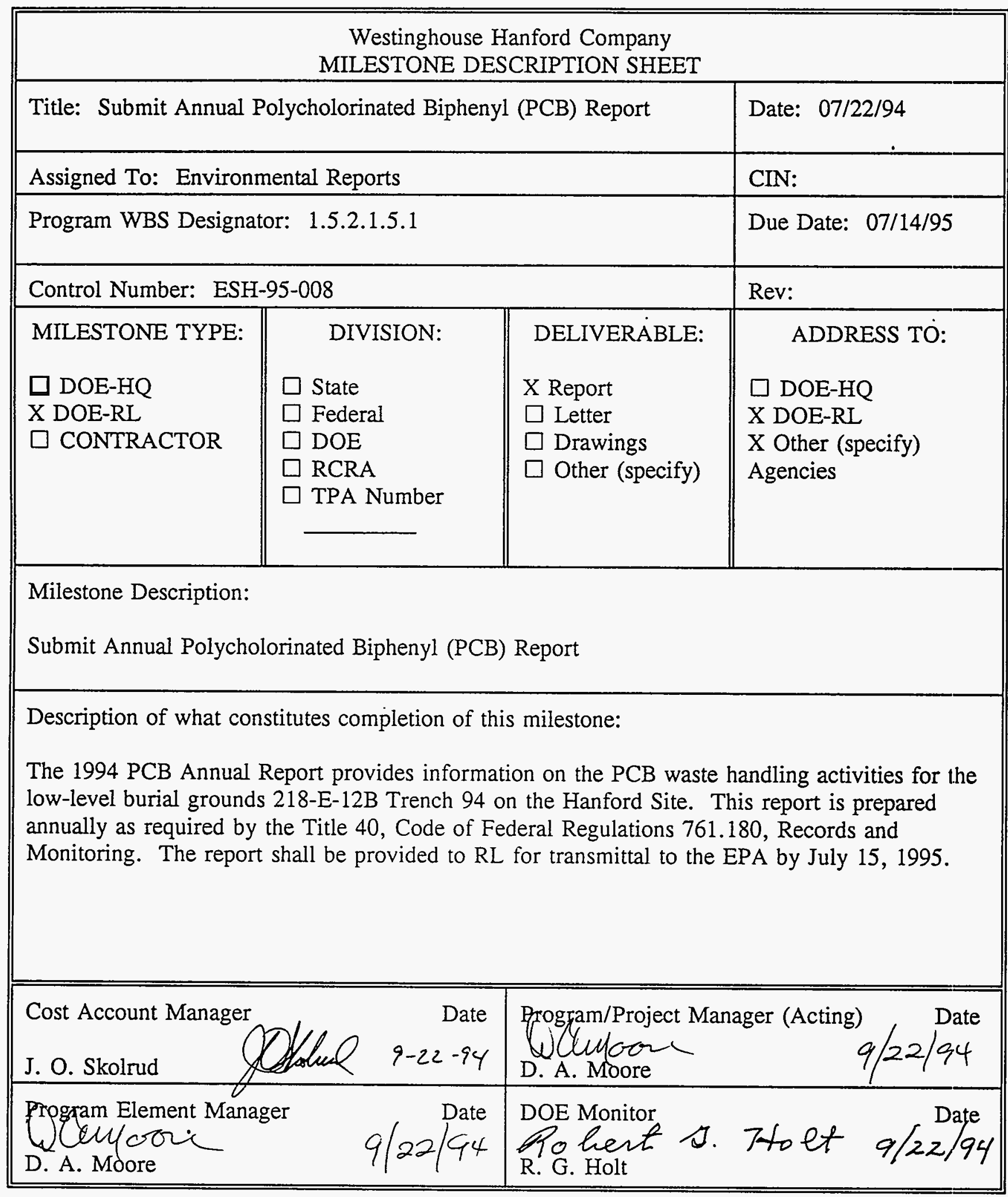




\begin{tabular}{|c|c|c|c|}
\hline \multicolumn{4}{|c|}{$\begin{array}{l}\text { Westinghouse Hanford Company } \\
\text { MILESTONE DESCRIPTION SHEET }\end{array}$} \\
\hline \multicolumn{3}{|c|}{ Title: Complete Laboratory Activity Toxic Use Report } & Date: $07 / 22 / 94$ \\
\hline \multicolumn{3}{|c|}{ Assigned To: Environmental Reports } & CIN: \\
\hline \multicolumn{3}{|c|}{ Program WBS Designator: $1 \cdot 5 \cdot 2 \cdot 1.5 .1$} & Due Date: $08 / 01 / 95$ \\
\hline \multicolumn{3}{|c|}{ Control Number: ESH-95-009 } & Rev: \\
\hline $\begin{array}{l}\text { MILESTONE TYPE: } \\
\text { X DOE-HQ } \\
\square \text { DOE-RL } \\
\square \text { CONTRACTOR }\end{array}$ & $\begin{aligned} & \text { DIVISION: } \\
& \\
& \text { State } \\
& \text { Federal } \\
& \text { DOE } \\
& \text { DCRA } \\
& \square \text { TPA Number }\end{aligned}$ & $\begin{array}{l}\text { DELIVERABLE: } \\
\text { X Report } \\
\square \text { Letter } \\
\square \text { Drawings } \\
\square \text { Other (specify) }\end{array}$ & $\begin{array}{l}\quad \text { ADDRESS TO: } \\
\text { X DOE-HQ } \\
\text { X DOE-RL } \\
\text { X Other (specify) } \\
\text { Agencies }\end{array}$ \\
\hline \multicolumn{4}{|c|}{$\begin{array}{l}\text { Milestone Description: } \\
\text { Complete Laboratory Activity Toxic Use Report }\end{array}$} \\
\hline \multicolumn{4}{|c|}{$\begin{array}{l}\text { Description of what constitutes completion of this milestone: } \\
\text { Prepare and submit annual Laboratory Activity Toxic Chemical Use Report for the Hanford Site } \\
\text { as required by DOE-HQ. Report is submitted to DOE-RL for transmittal to DOE-HQ to } \\
\text { evaluate applicability of the laboratory exemption to EPCRA Section } 313 \text { reporting requirements. }\end{array}$} \\
\hline \multicolumn{2}{|c|}{ Cost Account Manager } & \multicolumn{2}{|c|}{$\begin{array}{l}\text { Program/Project Manager (Acting) } \\
\text { Date } \\
\text { D. A. Moore } \\
9 / 22 / 94\end{array}$} \\
\hline \multicolumn{2}{|c|}{$\begin{array}{ll}\text { Date } \\
\text { D. A. Moore }\end{array} \quad 9 / 22 / 94$} & \multicolumn{2}{|c|}{$\begin{array}{l}\text { DOE Monitor } \\
\text { Po bert } s \text {. Tholt } 9 / 22 / 94 \\
\text { R. G. Holt }\end{array}$} \\
\hline
\end{tabular}




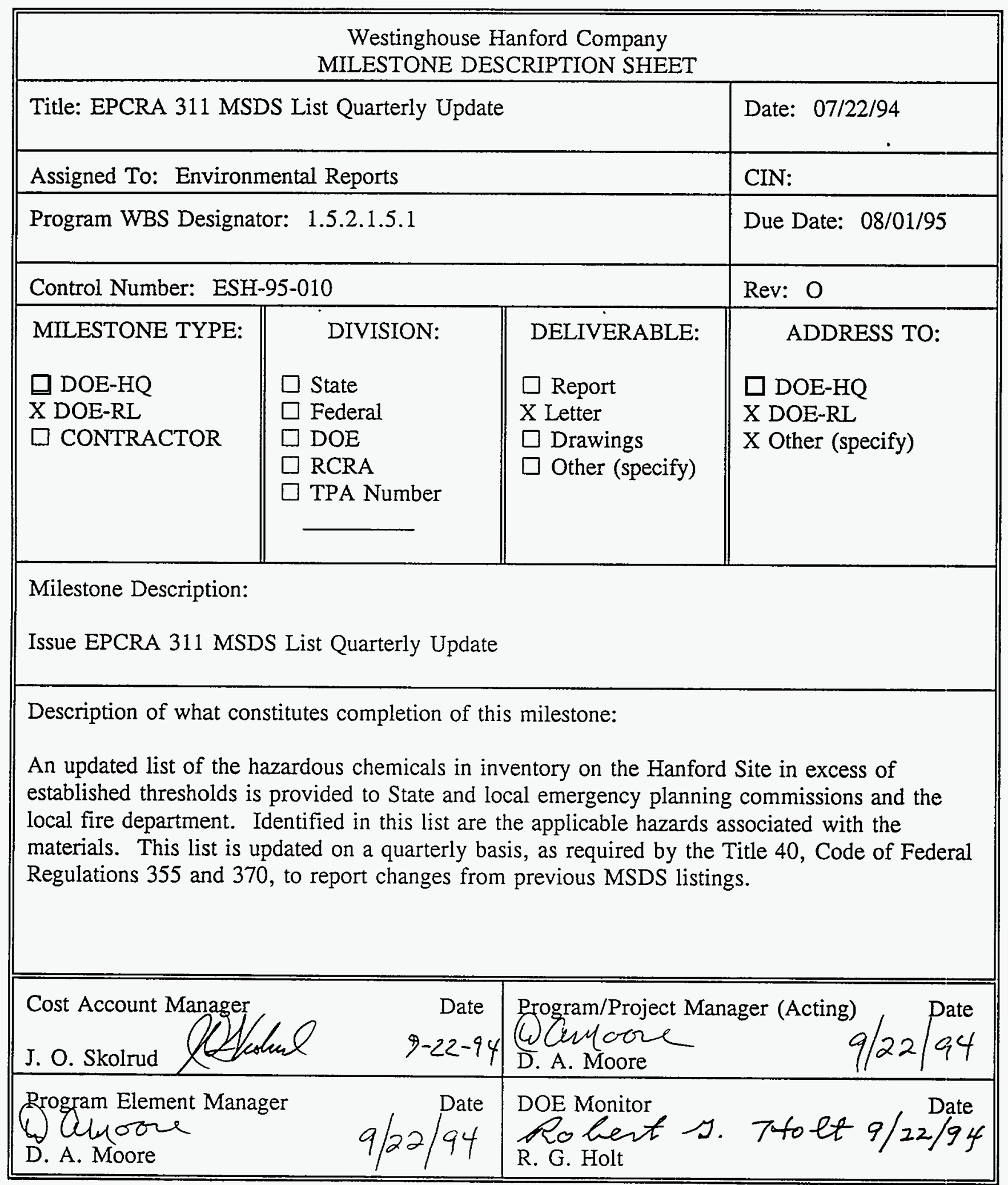




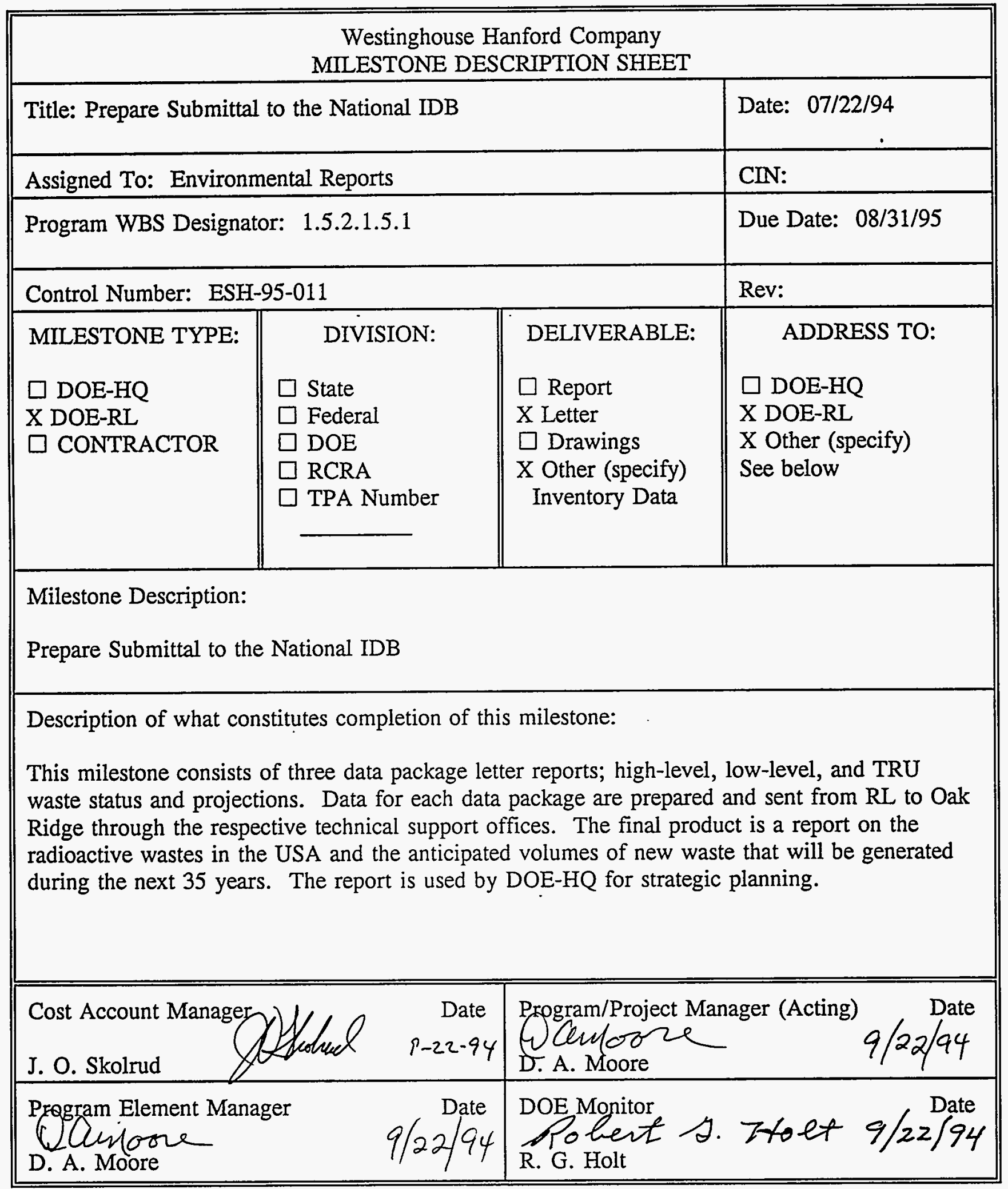




\begin{tabular}{|c|c|c|c|}
\hline \multicolumn{4}{|c|}{$\begin{array}{l}\text { Westinghouse Hanford Company } \\
\text { MILESTONE DESCRIPTION SHEET }\end{array}$} \\
\hline \multicolumn{3}{|c|}{ Title: Issue Annual LDR Status Report (M-26-01E) } & Date: $07 / 22 / 94$ \\
\hline \multicolumn{3}{|c|}{ Assigned To: Environmental Reports } & CIN: \\
\hline \multicolumn{3}{|c|}{ Program WBS Designator: $1 \cdot 5 \cdot 2 \cdot 1.5 .3$} & Due Date: $04 / 28 / 95$ \\
\hline \multicolumn{3}{|c|}{ Control Number: ESH-95-012 } & Rev: \\
\hline $\begin{array}{l}\text { MILESTONE TYPE: } \\
\square \text { DOE-HQ } \\
\text { X DOE-RL } \\
\square \text { CONTRACTOR }\end{array}$ & $\begin{array}{l}\quad \text { DIVISION: } \\
\square \text { State } \\
\square \text { Federal } \\
\square \text { DOE } \\
\square \text { RCRA } \\
\square \text { TPA Number }\end{array}$ & $\begin{array}{l}\text { DELIVERABLE: } \\
\square \text { Report } \\
\text { X Letter } \\
\square \text { Drawings } \\
\square \text { Other (specify) }\end{array}$ & $\begin{array}{l}\quad \text { ADDRESS TO: } \\
\square \text { DOE-HQ } \\
\text { X DOE-RL } \\
\text { X Other (specify) } \\
\text { Agencies }\end{array}$ \\
\hline \multicolumn{4}{|c|}{$\begin{array}{l}\text { Milestone Description: } \\
\text { Issue Annual LDR Status Report (M-26-01E) }\end{array}$} \\
\hline \multicolumn{4}{|c|}{$\begin{array}{l}\text { Description of what constitutes completion of this milestone: } \\
\text { Prepare and issue the } 1995 \text { update of the annual status report on the Hanford Site Land Disposal } \\
\text { Restrictions (LDR) for mixed wastes. This RL primary document, per the Tri-Party Agreement, } \\
\text { contains waste characterization information, storage data, treatment information and waste } \\
\text { reduction information for sixteen waste streams. Progress in achieving compliance with LDR } \\
\text { regulations since the previous status report is detailed and a work schedule is provided. This } \\
\text { report is provided as a Tri-Party agreement primary document to EPA and Ecology by April 30, } \\
\text { 1995. }\end{array}$} \\
\hline \multicolumn{2}{|c|}{ Jost Account Manager } & \multicolumn{2}{|c|}{$\begin{array}{l}\text { Program/Project Manager (Acting) } \\
\text { D Cufoore } \\
\text { D. A. Moore } \\
\text { D. Mate }\end{array}$} \\
\hline \multicolumn{2}{|c|}{$\begin{array}{ll}\text { Program Element Manager } & \text { Date } \\
\text { D. A. Moore } & 9 / 22 / 94\end{array}$} & \multicolumn{2}{|c|}{$\begin{array}{l}\text { DOE Monitor } \\
\text { Po hert } y \text {. Ztoef } 9 / 22 / 94 \\
\text { R. G. Holt }\end{array}$} \\
\hline
\end{tabular}


WHC-SP-1112

\begin{tabular}{|c|c|c|c|}
\hline \multicolumn{4}{|c|}{$\begin{array}{l}\text { Westinghouse Hanford Company } \\
\text { MILESTONE DESCRIPTION SHEET }\end{array}$} \\
\hline \multicolumn{3}{|c|}{ Title: Develop 3 Performance Measures/Indicators for P2 Progress } & Date: $07 / 22 / 94$ \\
\hline \multicolumn{3}{|c|}{ Assigned To: Reengineering Program Office } & CIN: \\
\hline \multicolumn{3}{|c|}{ Program WBS Designator: 1 -5.2.2.1.1 } & Due Date: $03 / 30 / 95$ \\
\hline \multicolumn{3}{|c|}{ Control Number: ESH-95-013 } & Rev: \\
\hline $\begin{array}{l}\text { MILESTONE TYPE: } \\
\square \text { DOE-HQ } \\
\text { X DOE-RL } \\
\square \text { CONTRACTOR }\end{array}$ & $\begin{array}{l}\quad \text { DIVISION: } \\
\square \text { State } \\
\square \text { Federal } \\
\square \text { DOE } \\
\square \text { RCRA } \\
\square \text { TPA Number }\end{array}$ & $\begin{array}{l}\text { DELIVERABLE: } \\
\text { } \square \text { Report } \\
\square \text { Letter } \\
\square \text { Drawings } \\
\text { X Other (specify) } \\
\text { See below }\end{array}$ & $\begin{array}{l}\text { ADDRESS TO: } \\
\square \text { DOE-HQ } \\
\text { X DOE-RL } \\
\square \text { Other (specify) }\end{array}$ \\
\hline \multicolumn{4}{|c|}{$\begin{array}{l}\text { Milestone Description: } \\
\text { Develop } 3 \text { Performance Measures/Indicators for P2 Progress }\end{array}$} \\
\hline \multicolumn{4}{|c|}{$\begin{array}{l}\text { Description of what constitutes completion of this milestone: } \\
\text { Three performance measures/indicators submitted to RL by due date. }\end{array}$} \\
\hline \multicolumn{2}{|c|}{ 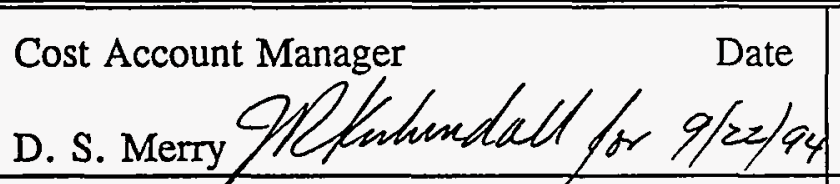 } & \multicolumn{2}{|c|}{$\begin{array}{l}\text { Program/Project Manager (Acting) } \\
\text { D. Ampore Date } \\
\text { D. Moore } 9 / 22 / 94\end{array}$} \\
\hline \multicolumn{2}{|c|}{$\begin{array}{l}\text { Prgiam Element Manager } \\
\text { D. A. Moore }\end{array}$} & \multicolumn{2}{|c|}{$\begin{array}{l}\text { DOE Monitorh AleuB. Bagan a|vrat } \\
\text { E. B. Dagan }\end{array}$} \\
\hline
\end{tabular}


WHC-SP-1112

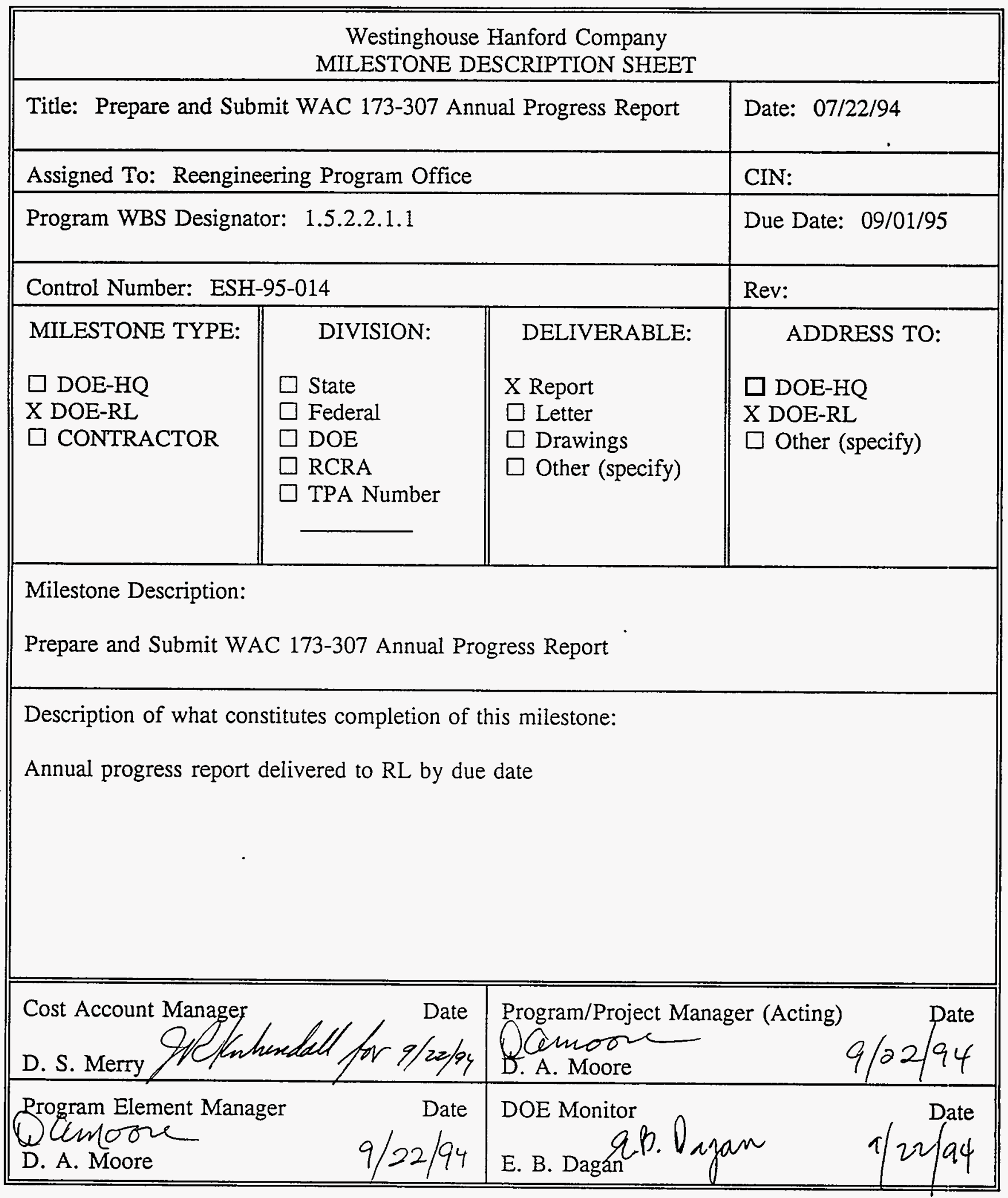




\begin{tabular}{|l||l|||}
\hline \multicolumn{3}{|c||}{ Westinghouse Hanford Company } \\
MLESTONE DESCRIPTION SHEET
\end{tabular}


FY 1996 Milestone Description Sheets 


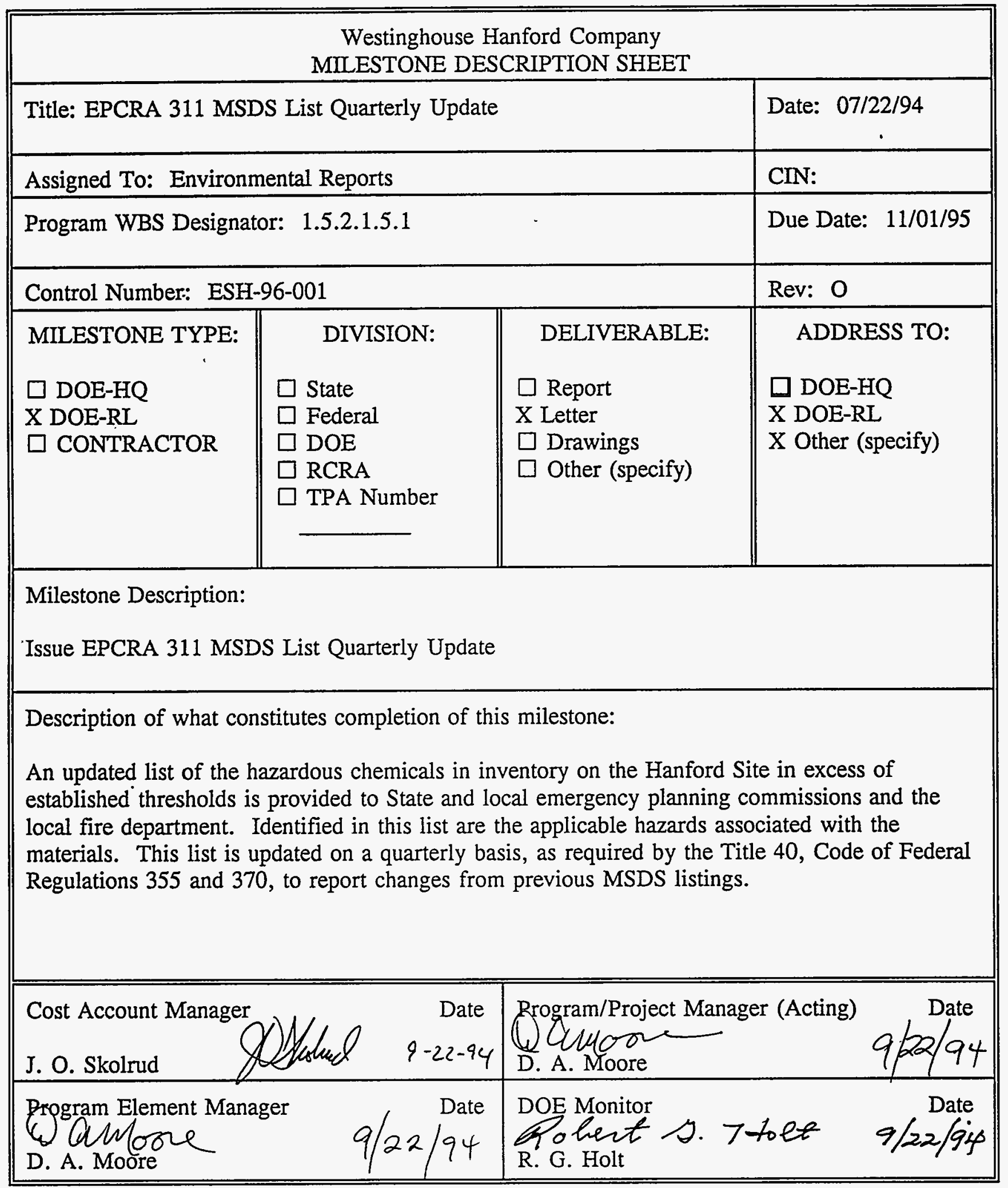




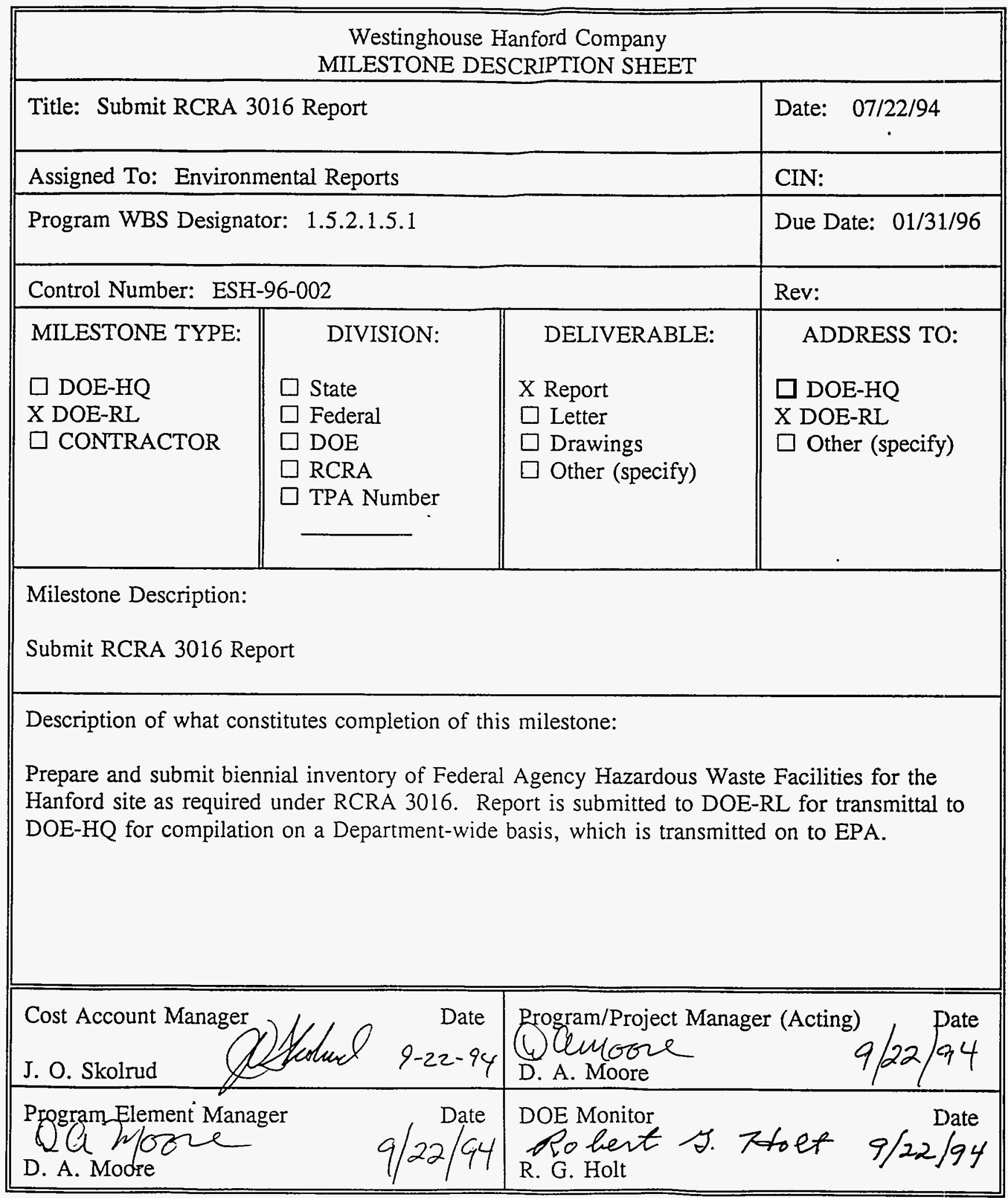




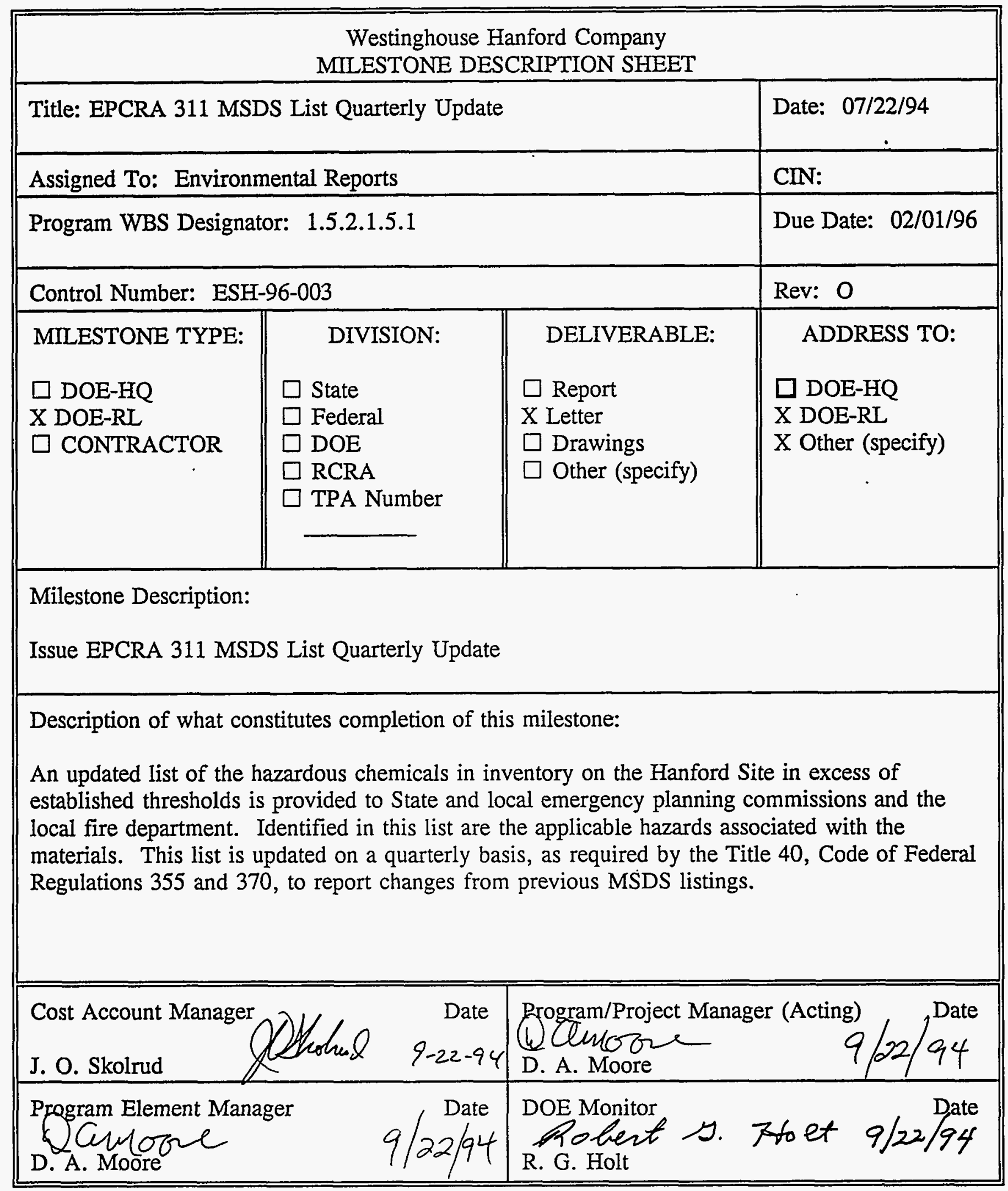




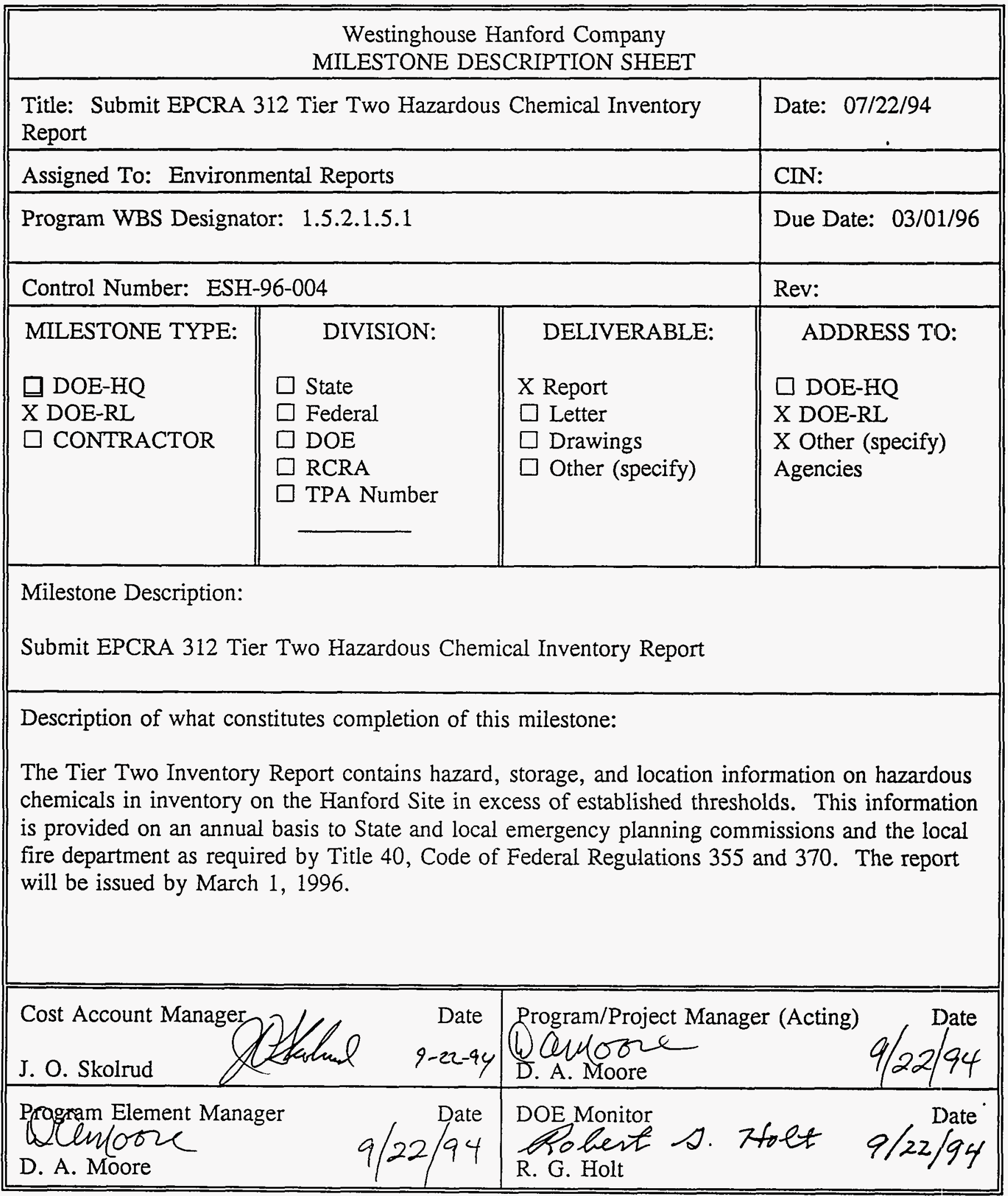




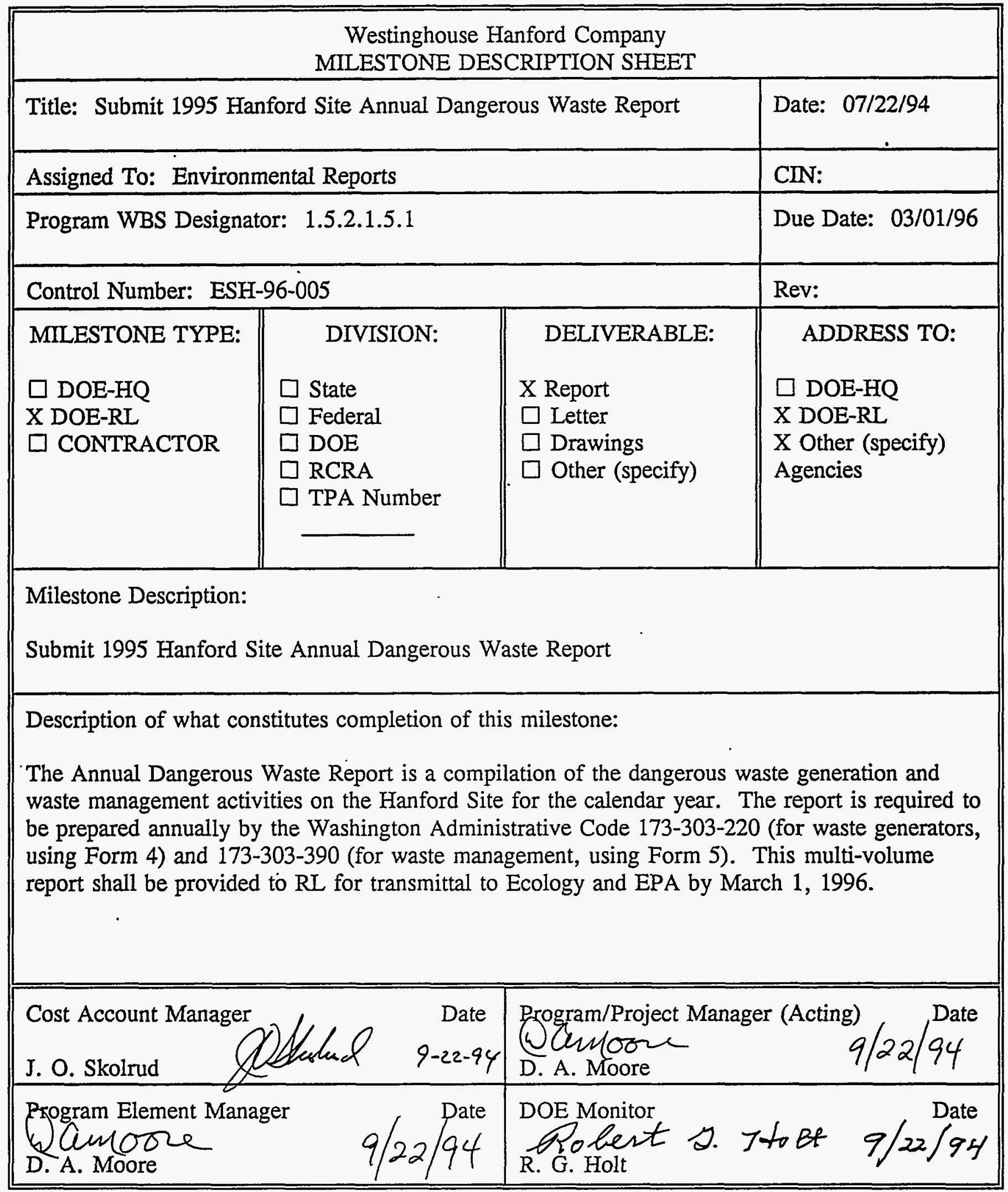




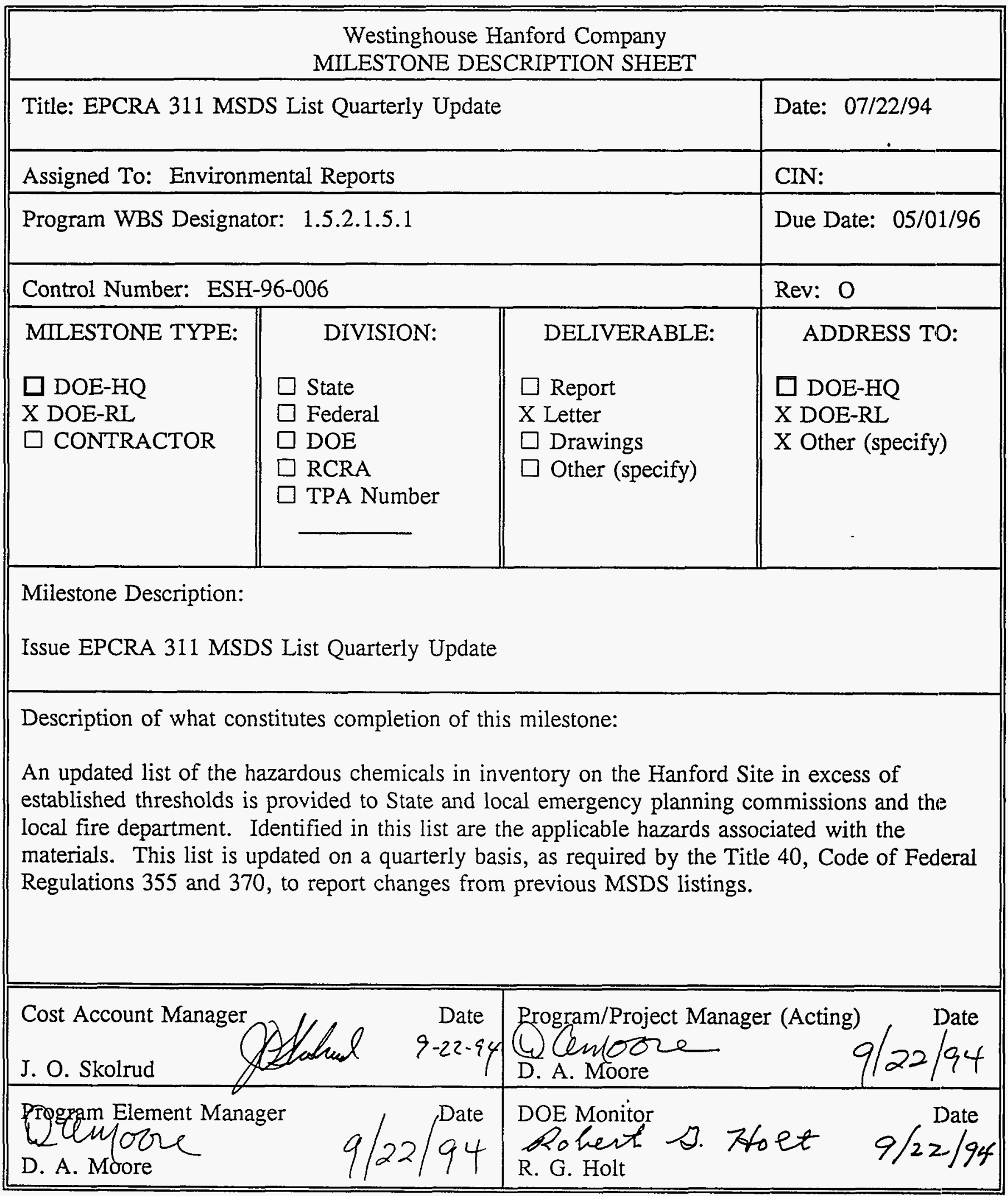




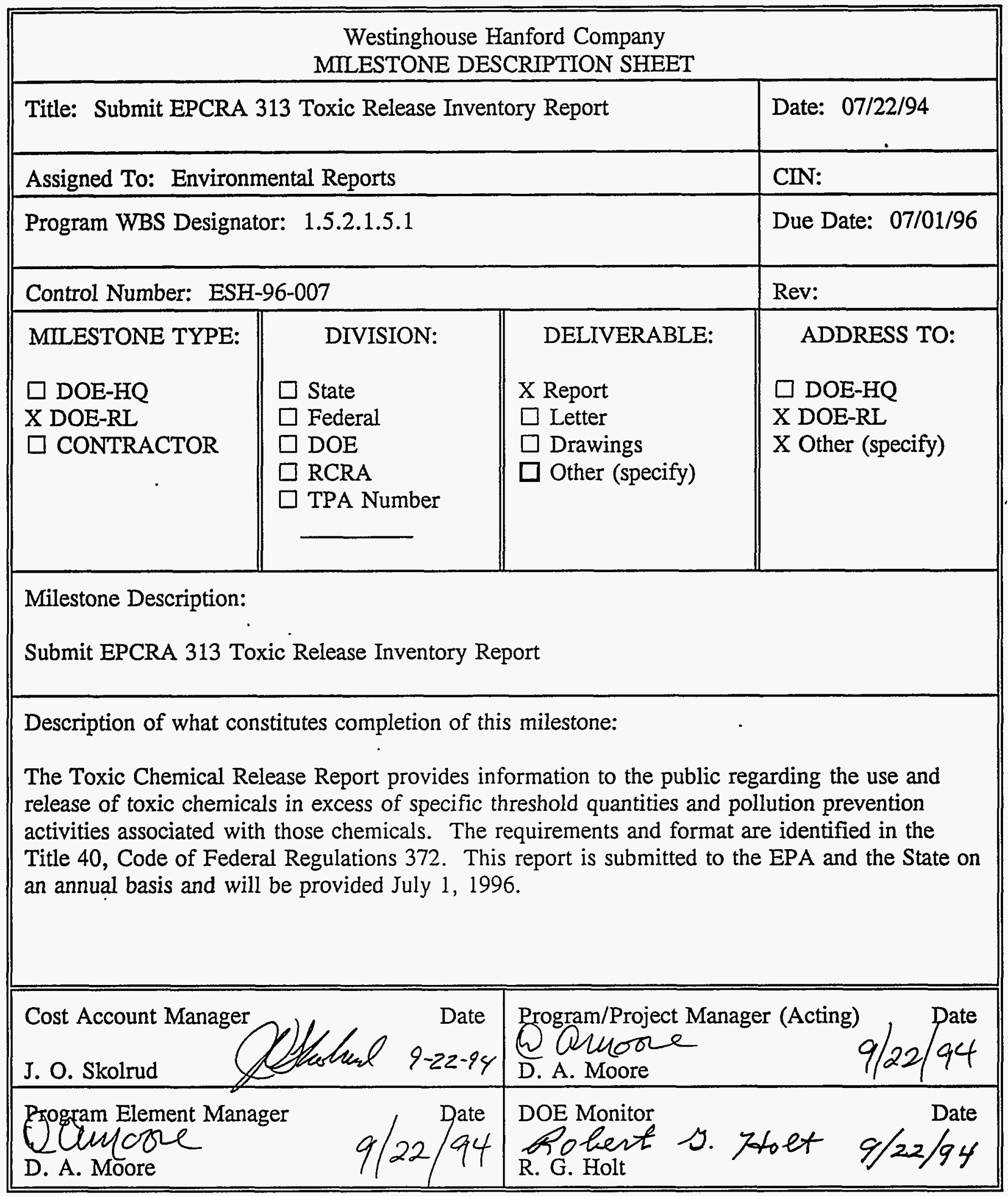




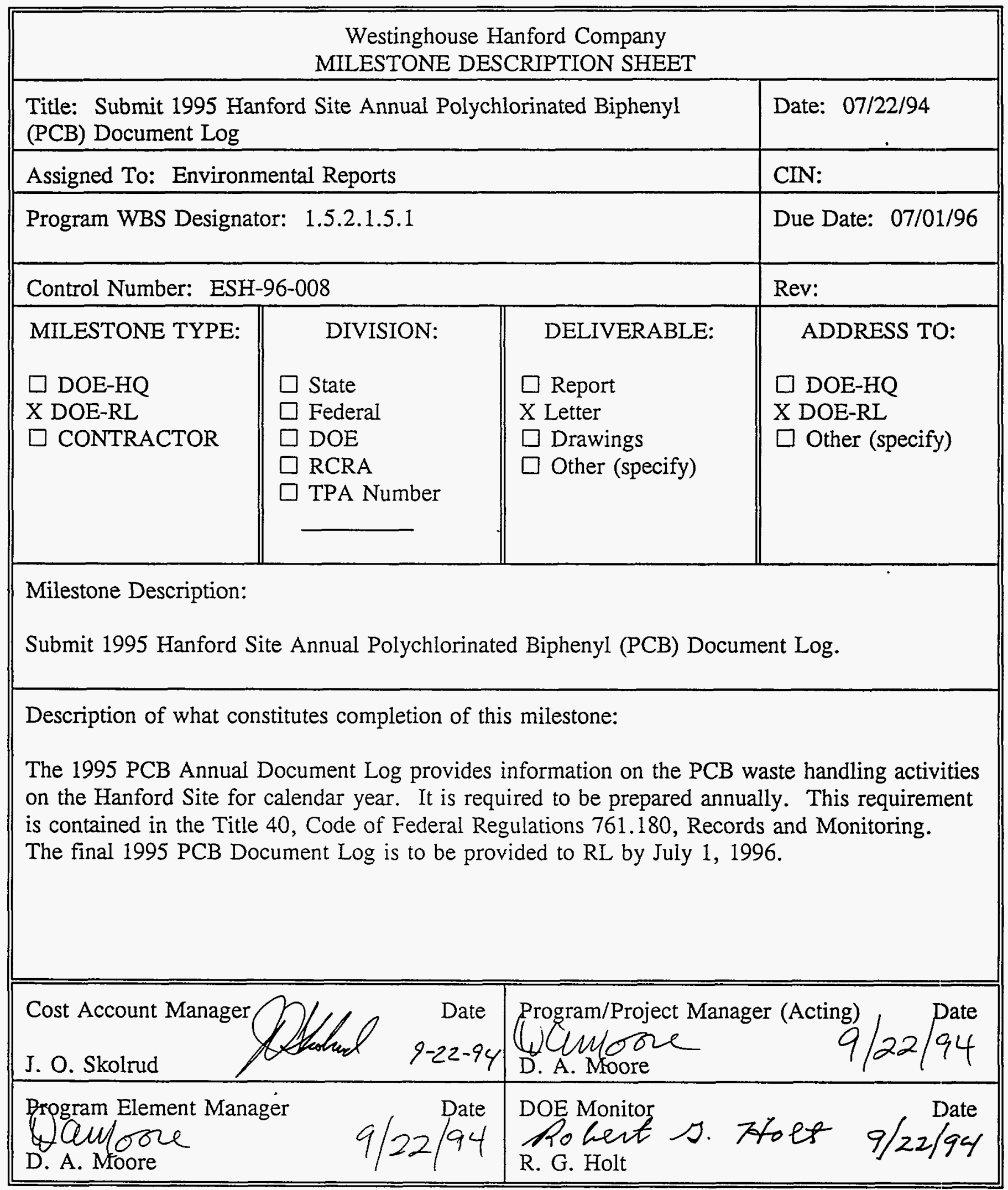




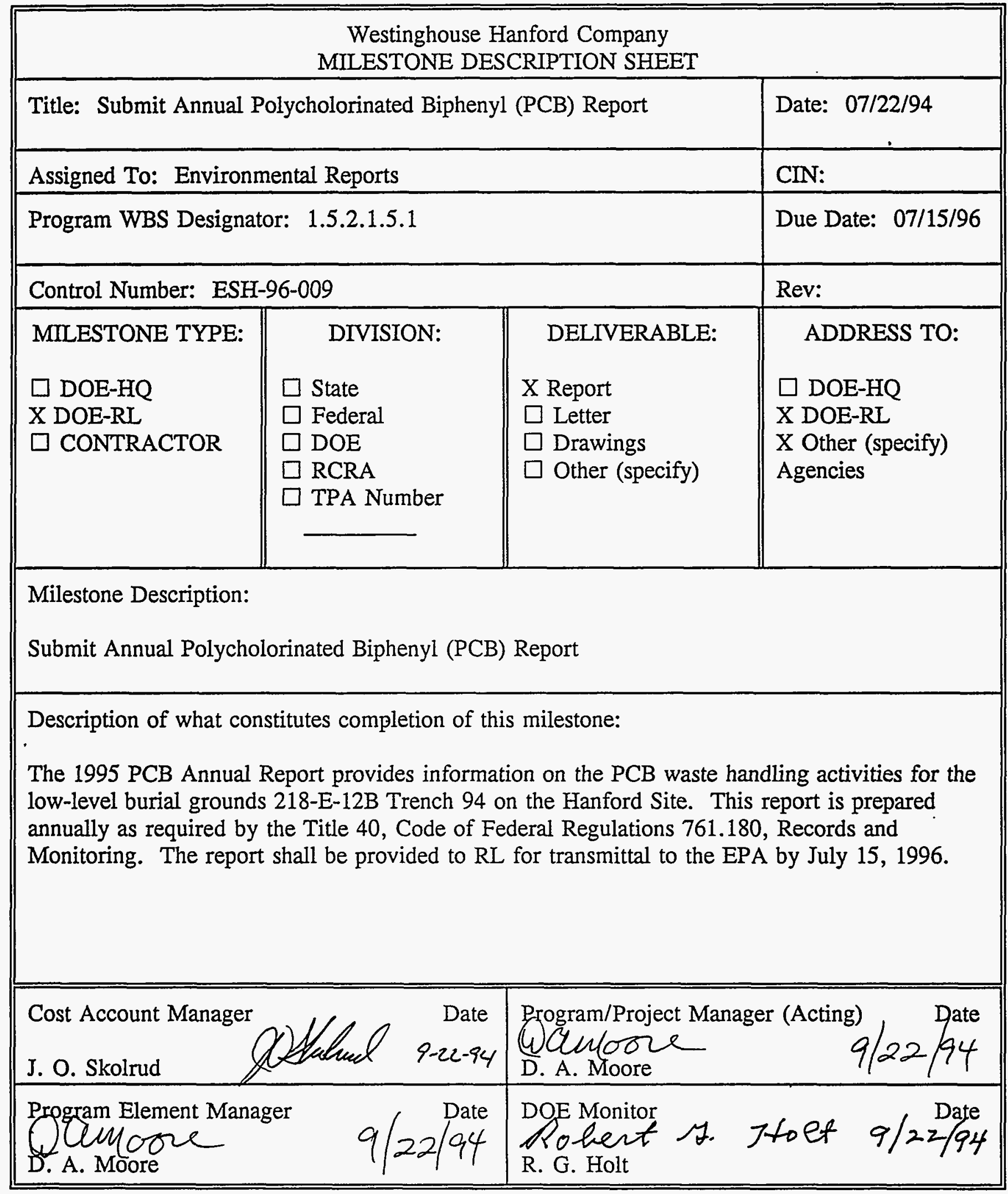




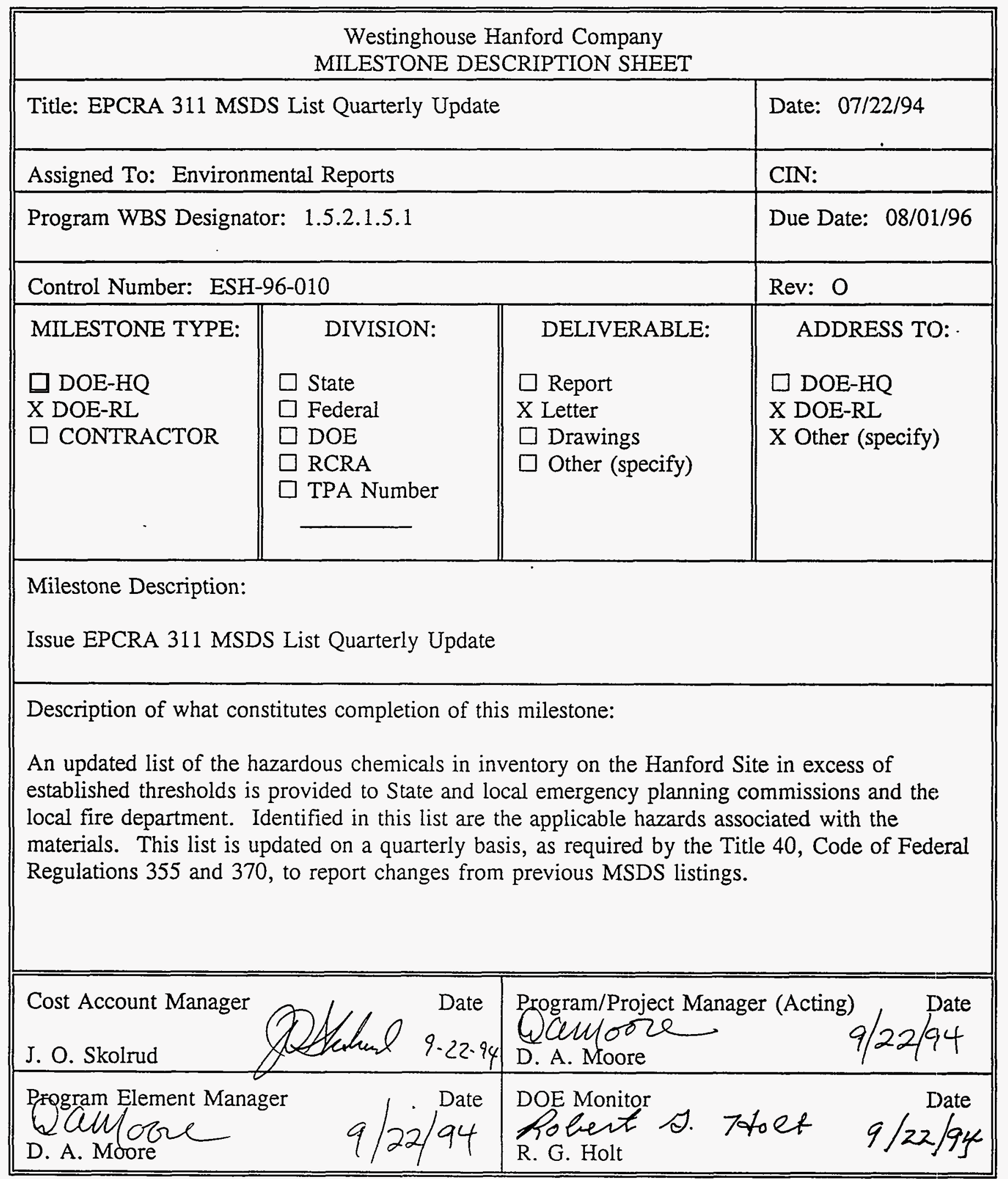




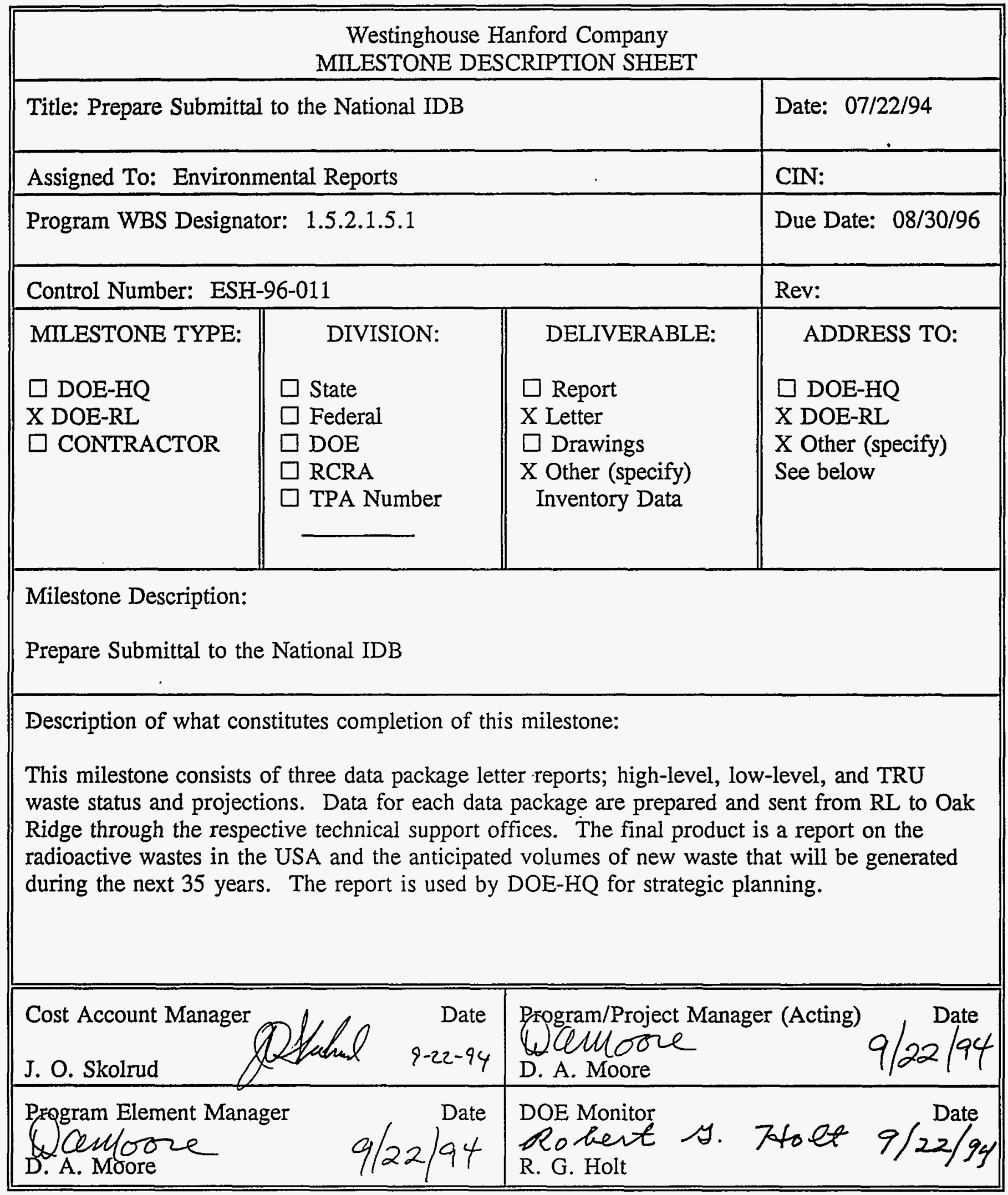




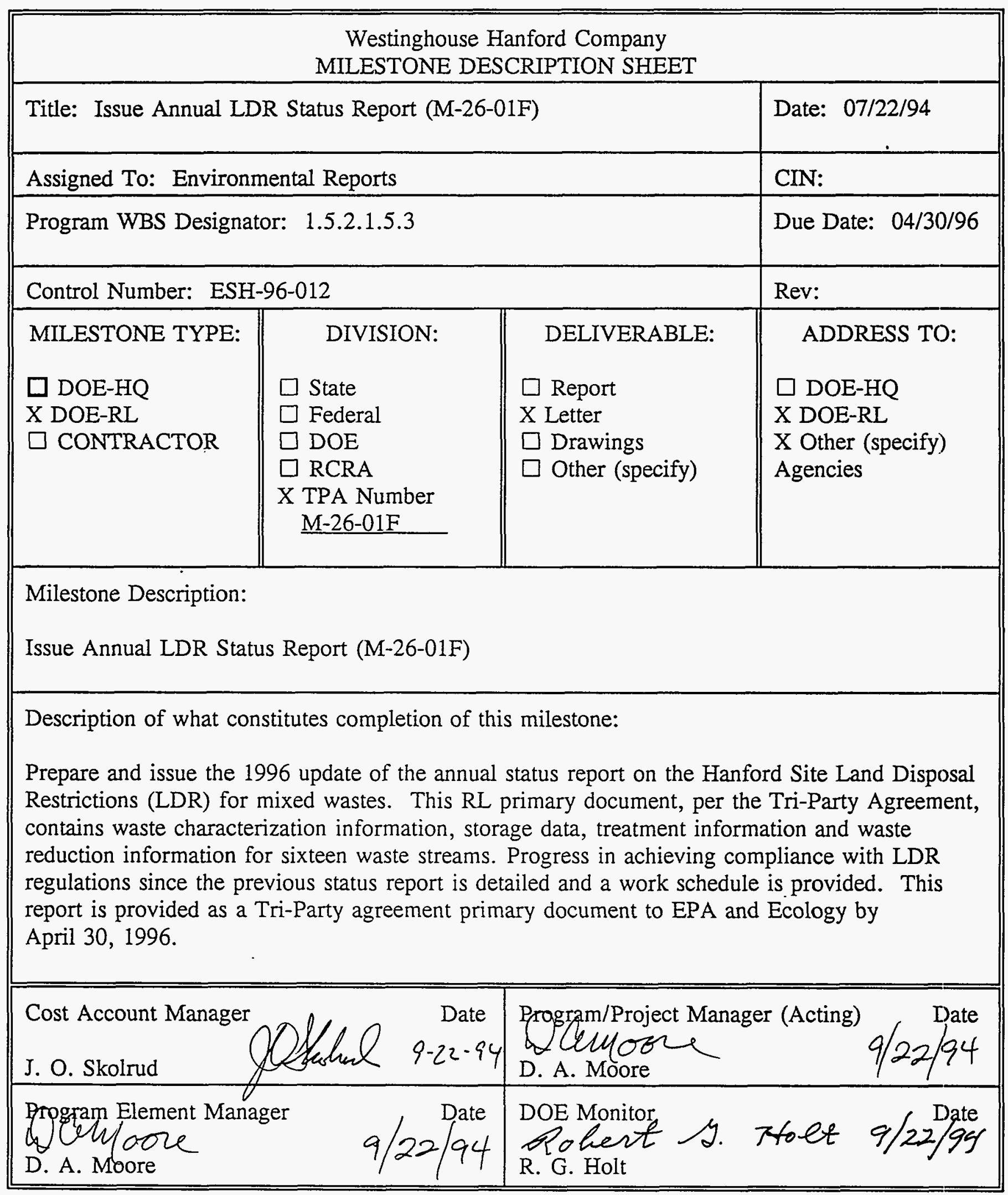




\begin{tabular}{|c|c|c|c|}
\hline \multicolumn{4}{|c|}{$\begin{array}{l}\text { Westinghouse Hanford Company } \\
\text { MILESTONE DESCRIPTION SHEET }\end{array}$} \\
\hline \multicolumn{3}{|c|}{$\begin{array}{l}\text { Title: Submit Part B Permit Applications or Closure Plans for All } \\
\text { RCRA }\end{array}$} & Date: $\quad 07 / 22 / 94$ \\
\hline \multicolumn{3}{|c|}{ Assigned To: RCRA Permitting } & CIN: \\
\hline \multicolumn{3}{|c|}{ Program WBS Designator: $1 \cdot 5 \cdot 2 \cdot 1 \cdot 7.8$} & Due Date: $05 / 31 / 96$ \\
\hline \multicolumn{3}{|c|}{ Control Number: ESH-96-013 } & Rev: \\
\hline $\begin{array}{l}\text { MILESTONE TYPE: } \\
\square \text { DOE-HQ } \\
\text { X DOE-RL } \\
\square \text { CONTRACTOR }\end{array}$ & $\begin{aligned} & \text { DIVISION: } \\
& \square \text { State } \\
& \text { Federal } \\
& \square \text { DOE } \\
& \square \text { RCRA } \\
& \text { X TPA Number } \text { M-20-00 } \\
&\end{aligned}$ & $\begin{array}{l}\text { DELIVERABLE: } \\
\square \text { Report } \\
\text { X Letter } \\
\square \text { Drawings } \\
\square \text { Other (specify) }\end{array}$ & $\begin{array}{l}\quad \text { ADDRESS TO: } \\
\square \text { DOE-HQ } \\
\text { X DOE-RL } \\
\text { X Other (specify) } \\
\text { EPA, Ecology }\end{array}$ \\
\hline \multicolumn{4}{|c|}{$\begin{array}{l}\text { Milestone Description: } \\
\text { Submit Part B Permit Applications or Closure Plans for All RCRA }\end{array}$} \\
\hline \multicolumn{4}{|c|}{$\begin{array}{l}\text { Description of what constitutes completion of this milestone: } \\
\text { Submit Part B Permit Applications or Closure Plans for All RCRA TSD units }\end{array}$} \\
\hline \multicolumn{2}{|l|}{$\begin{array}{l}\text { Cost Account Manager } \\
\text { W. Cellooce for } \\
\text { s.M. Price }\end{array}$} & \multicolumn{2}{|c|}{$\begin{array}{l}\text { Program/Project Manager (Acting) } \\
\text { Date } \\
\text { D. A. Moore } 9 / 22 / 94\end{array}$} \\
\hline \multicolumn{2}{|c|}{$\begin{array}{l}\text { Program Element Manager } \\
\text { D. A. Moore }\end{array}$} & \multicolumn{2}{|c|}{$\begin{array}{l}\text { DOE Monitor } \\
\text { Po hert } 0 \text {. Folet } 9 / 22 / 94 \\
\text { R. G. Holt }\end{array}$} \\
\hline
\end{tabular}




\begin{tabular}{|c|c|c|c|}
\hline \multicolumn{4}{|c|}{$\begin{array}{l}\text { Westinghouse Hanford Company } \\
\text { MILESTONE DESCRIPTION SHEET }\end{array}$} \\
\hline \multicolumn{3}{|c|}{$\begin{array}{l}\text { Title: Prepare and Submit EPA Biennial Report on Waste } \\
\text { Minimization }\end{array}$} & Date: $07 / 22 / 94$ \\
\hline \multicolumn{3}{|c|}{ Assigned To: Reengineering Program Office } & CIN: \\
\hline \multicolumn{3}{|c|}{ Program WBS Designator: 1 -5.2.2.1.1 } & Due Date: $03 / 29 / 96$ \\
\hline \multicolumn{3}{|c|}{ Control Number: ESH-96-014 } & Rev: \\
\hline $\begin{array}{l}\text { MILESTONE } \\
\text { TYPE: } \\
\square \text { DOE-HQ } \\
\text { X DOE-RL } \\
\square \text { CONTRACTOR }\end{array}$ & $\begin{array}{l}\quad \text { DIVISION: } \\
\square \text { State } \\
\square \text { Federal } \\
\square \text { DOE } \\
\square \text { RCRA } \\
\square \text { TPA Number }\end{array}$ & $\begin{array}{l}\text { DELIVERABLE: } \\
\text { X Report } \\
\square \text { Letter } \\
\square \text { Drawings } \\
\square \text { Other (specify) }\end{array}$ & $\begin{array}{l}\text { ADDRESS TO: } \\
\square \text { DOE-HQ } \\
\text { X DOE-RL } \\
\square \text { Other (specify) }\end{array}$ \\
\hline \multicolumn{4}{|c|}{$\begin{array}{l}\text { Milestone Description: } \\
\text { Prepare and Submit EPA Biennial Report on Waste Minimization }\end{array}$} \\
\hline \multicolumn{4}{|c|}{$\begin{array}{l}\text { Description of what constitutes completion of this milestone: } \\
\text { EPA Biennial report submitted to RL by due date. }\end{array}$} \\
\hline \multicolumn{2}{|c|}{ 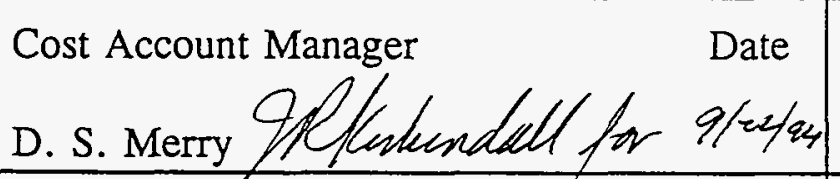 } & \multicolumn{2}{|c|}{$\begin{array}{l}\text { Program/Project Manager (Acting) } \\
\text { Lamoore } \\
\text { D. A. Moore }\end{array}$} \\
\hline \multicolumn{2}{|c|}{$\begin{array}{l}\text { Prggram Element Manager } \\
\text { Cem Doone } \\
\text { D. A. Moore }\end{array}$} & \multicolumn{2}{|c|}{$\begin{array}{l}\text { DOE Monitor } \\
\text { E. B. Dagan }\end{array}$} \\
\hline
\end{tabular}




\begin{tabular}{|c|c|c|c|}
\hline \multicolumn{4}{|c|}{$\begin{array}{l}\text { Westinghouse Hanford Company } \\
\text { MILESTONE DESCRIPTION SHEET }\end{array}$} \\
\hline \multicolumn{3}{|c|}{ Title: Develop 3 Performance Measures/Indicators for P2 Program } & Date: $07 / 22 / 94$ \\
\hline \multicolumn{3}{|c|}{ Assigned To: Reengineering Program Office } & CIN: \\
\hline \multicolumn{3}{|c|}{ Program WBS Designator: 1 .5.2.2.1.1 } & Due Date: $04 / 01 / 96$ \\
\hline \multicolumn{3}{|c|}{ Control Number: ESH-96-015 } & Rev: \\
\hline $\begin{array}{l}\text { MILESTONE } \\
\text { TYPE: } \\
\square \text { DOE-HQ } \\
\text { X DOE-RL } \\
\square \text { CONTRACTOR }\end{array}$ & $\begin{aligned} & \text { DIVISION: } \\
& \\
& \text { State } \\
\square & \text { Federal } \\
& \text { DOE } \\
& \text { RCRA } \\
& \square \text { TPA Number }\end{aligned}$ & $\begin{array}{l}\text { DELIVERABLE: } \\
\square \text { Report } \\
\square \text { Letter } \\
\square \text { Drawings } \\
\text { X Other (specify) }\end{array}$ & $\begin{array}{l}\text { ADDRESS TO: } \\
\square \text { DOE-HQ } \\
\text { X DOE-RL } \\
\square \text { Other (specify) }\end{array}$ \\
\hline \multicolumn{4}{|c|}{$\begin{array}{l}\text { Milestone Description: } \\
\text { Develop } 3 \text { Performance Measures/Indicators for P2 Program }\end{array}$} \\
\hline \multicolumn{4}{|c|}{$\begin{array}{l}\text { Description of what constitutes completion of this milestone: } \\
\text { Three performance/indicators submitted to RL by due date. }\end{array}$} \\
\hline \multicolumn{2}{|c|}{$\begin{array}{ll}\text { Cost Account Manager } & \text { Date } \\
\text { D. S. Merry Offuntemelad } q / 22 / 94\end{array}$} & \multicolumn{2}{|c|}{$\begin{array}{l}\text { Program/Project Manager (Acting) } \\
\text { Wemoore } \\
\text { D. A. Moore } \\
9 / 22 / 94\end{array}$} \\
\hline \multicolumn{2}{|c|}{$\begin{array}{l}\text { Program Element Manager } \\
\text { Qunozre } \\
\text { D. A. Moore }\end{array}$} & \multicolumn{2}{|c|}{$\begin{array}{l}\text { DOE Monitor } \\
\text { E. B. Dagan }\end{array}$} \\
\hline
\end{tabular}


WHC-SP-1112

\begin{tabular}{|c|c|c|c|}
\hline \multicolumn{4}{|c|}{$\begin{array}{l}\text { Westinghouse Hanford Company } \\
\text { MILESTONE DESCRIPTION SHEET }\end{array}$} \\
\hline \multicolumn{3}{|c|}{ Title: Prepare and Submit WAC 173-307 Annual Progress Report } & Date: $07 / 22 / 94$ \\
\hline \multicolumn{3}{|c|}{ Assigned To: Reengineering Program Office } & CIN: \\
\hline \multicolumn{3}{|c|}{ Program WBS Designator: $1 \cdot 5 \cdot 2 \cdot 2.1 .1$} & Due Date: $08 / 30 / 96$ \\
\hline \multicolumn{3}{|c|}{ Control Number: ESH-96-016 } & Rev: \\
\hline $\begin{array}{l}\text { MILESTONE } \\
\text { TYPE: } \\
\square \text { DOE-HQ } \\
\text { X DOE-RL } \\
\square \text { CONTRACTOR }\end{array}$ & $\begin{aligned} & \text { DIVISION: } \\
& \square \text { State } \\
& \square \text { Federal } \\
& \square \text { DOE } \\
& \quad \text { RCRA } \\
& \square \text { TPA Number }\end{aligned}$ & $\begin{array}{l}\text { DELIVERABLE: } \\
\text { X Report } \\
\square \text { Letter } \\
\square \text { Drawings } \\
\square \text { Other (specify) }\end{array}$ & $\begin{array}{l}\text { ADDRESS TO: } \\
\square \text { DOE-HQ } \\
\text { X DOE-RL } \\
\square \text { Other (specify) }\end{array}$ \\
\hline \multicolumn{4}{|c|}{$\begin{array}{l}\text { Milestone Description: } \\
\text { Prepare and Submit WAC 173-307 Annual Progress Report }\end{array}$} \\
\hline \multicolumn{4}{|c|}{$\begin{array}{l}\text { Description of what constitutes completion of this milestone: } \\
\text { Annual progress report delivered to RL by due date. }\end{array}$} \\
\hline \multicolumn{2}{|c|}{$\begin{array}{ll}\text { Cost Account Manager } & \text { Date } \\
\text { D. S. Merry Mfflatundall q/rz/s4 }\end{array}$} & \multicolumn{2}{|c|}{$\begin{array}{l}\text { Program/Project Manager (Acting) } \\
\text { (Q) Date } \\
\text { D. A. Moore }\end{array}$} \\
\hline \multicolumn{2}{|c|}{$\begin{array}{ll}\text { Program Element Manager } & \text { Date } \\
\text { Q0 Woore } & 9 / 22 / 94\end{array}$} & \multicolumn{2}{|c|}{$\begin{array}{l}\text { DOE Monitor } \\
\text { E. B. Dagan }\end{array}$} \\
\hline
\end{tabular}




\begin{tabular}{||l|l||}
\hline \multicolumn{3}{|c||}{ Westinghouse Hanford Company } \\
MILESTONE DESCRIPTION SHEET
\end{tabular}


FY 1997 Milestone Description Sheets 


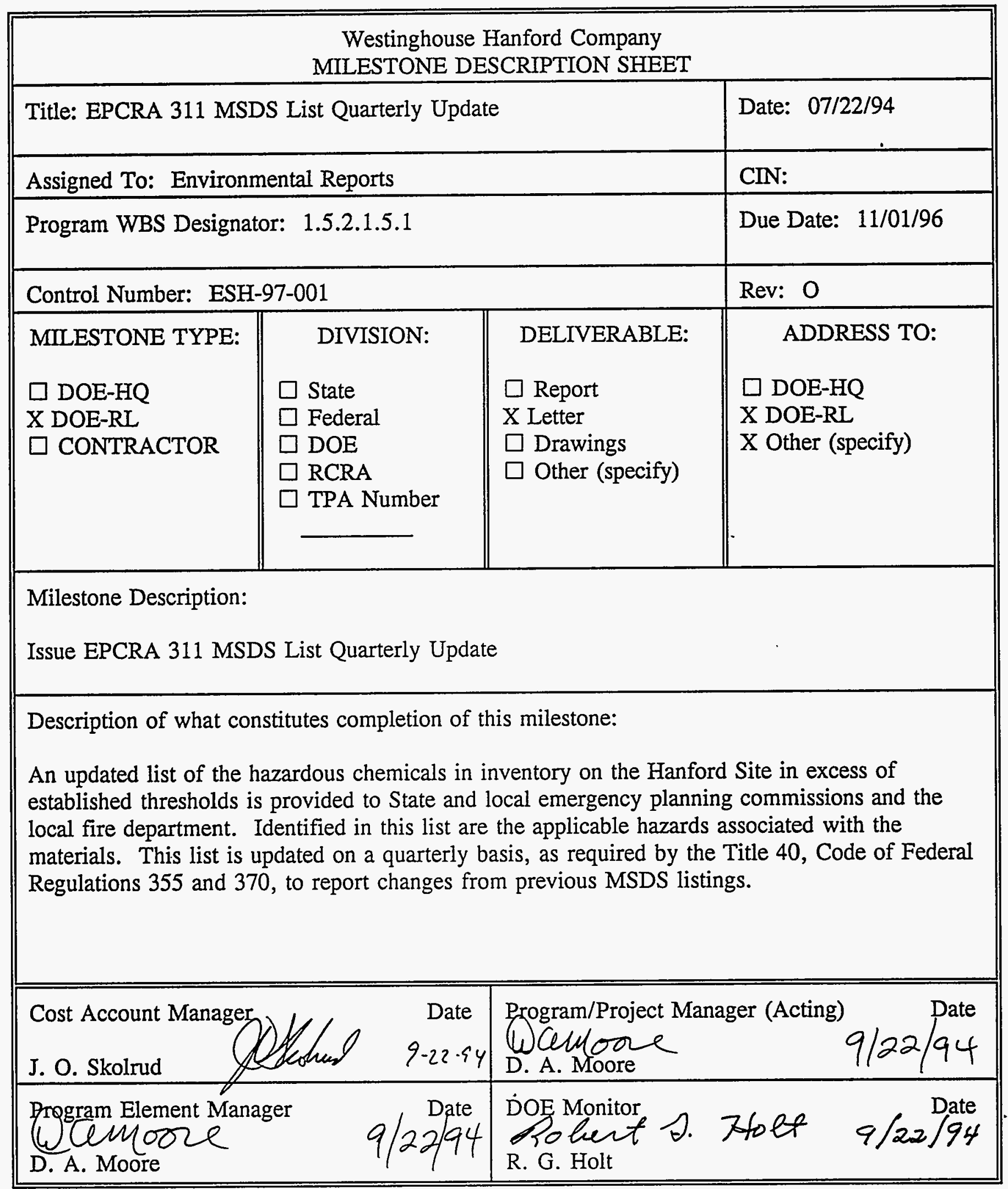


WHC-SP-1112

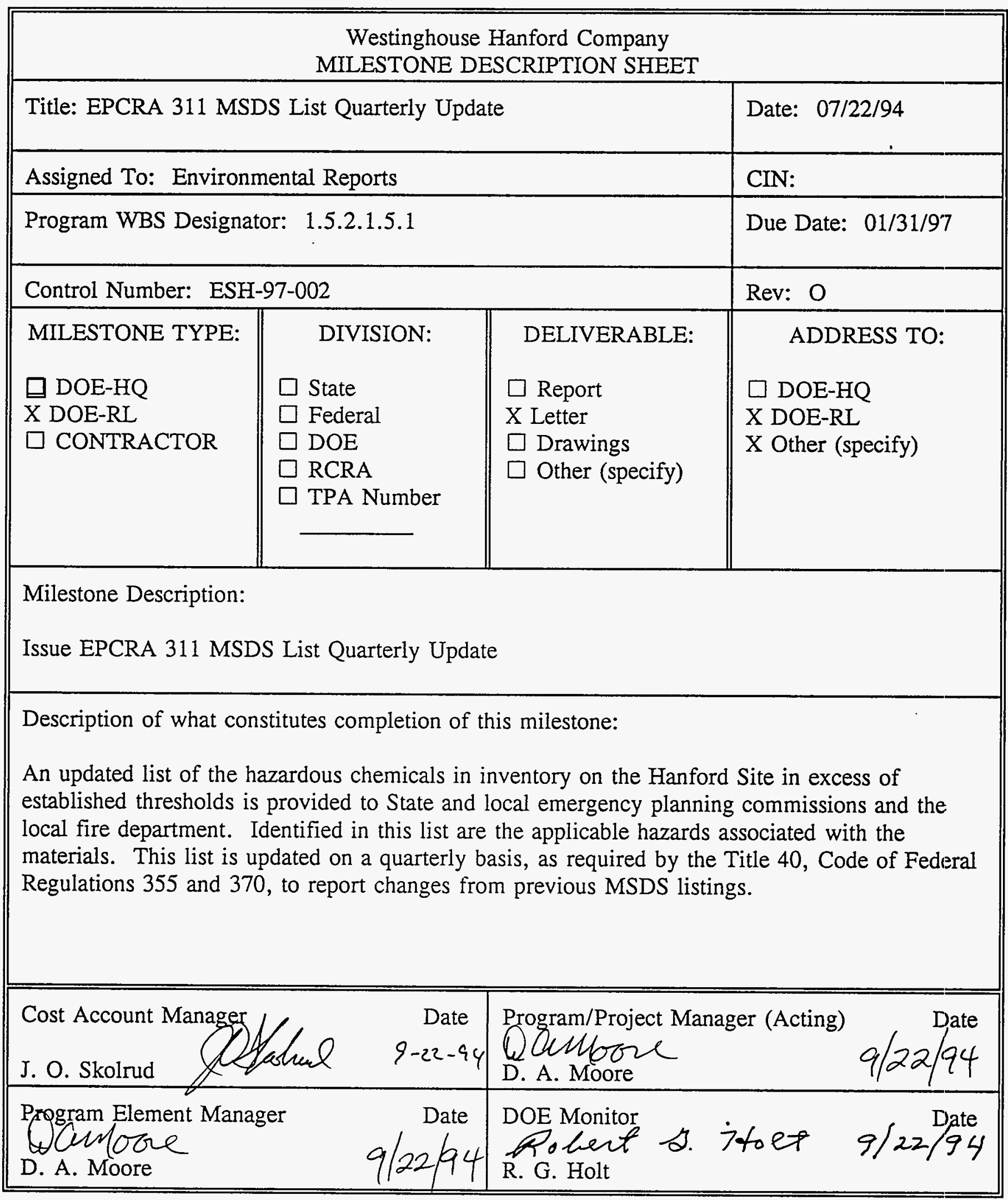




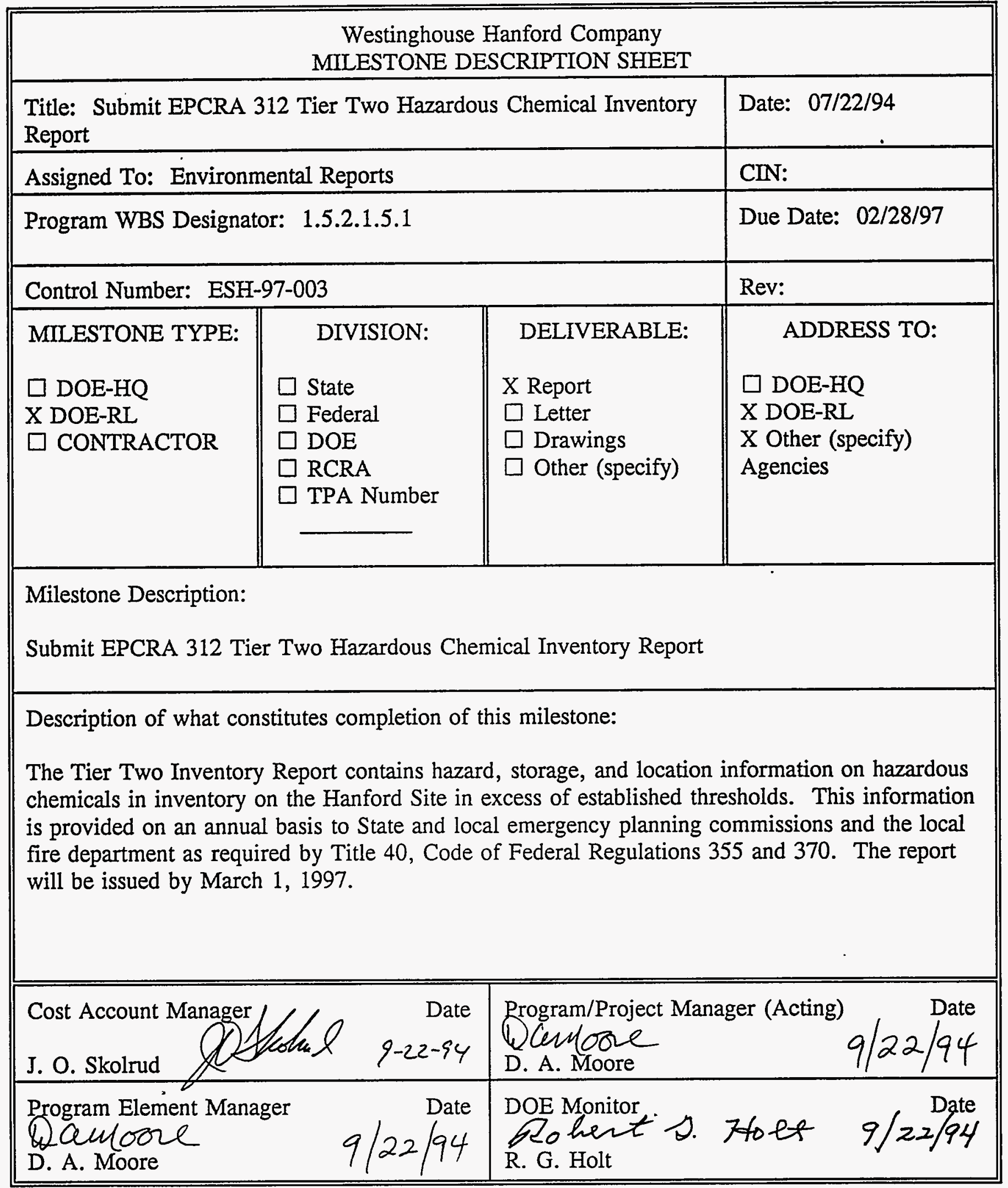




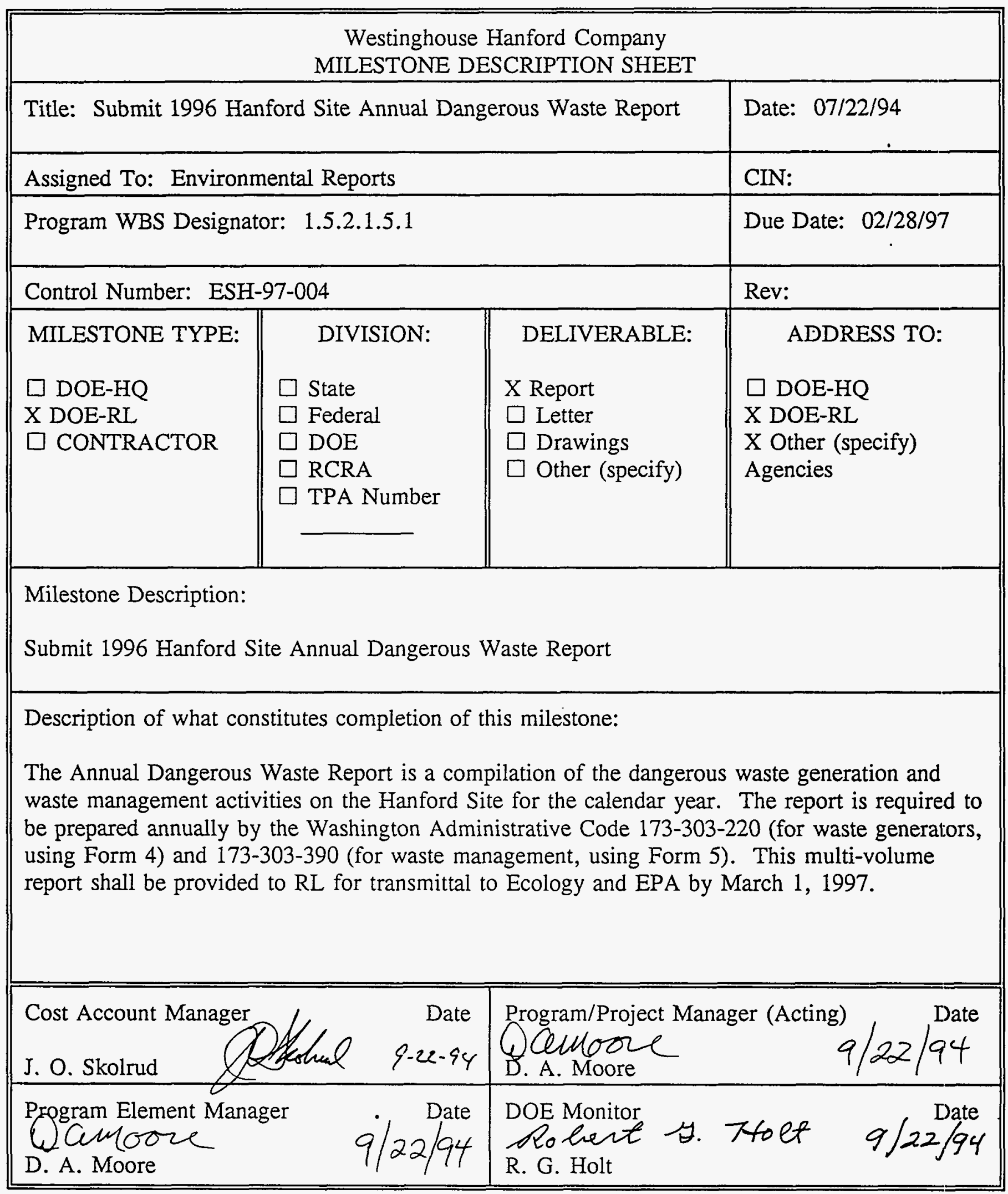




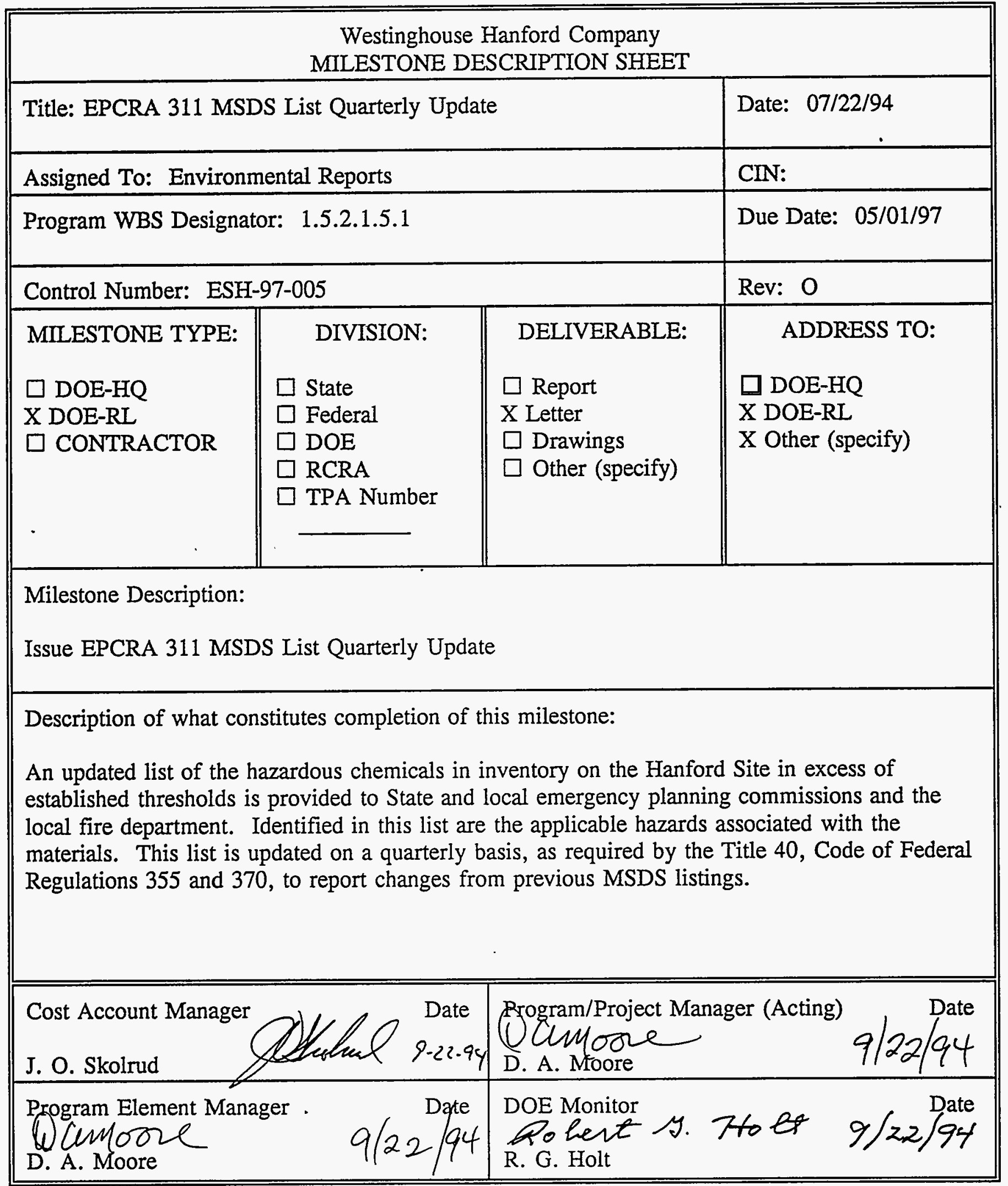




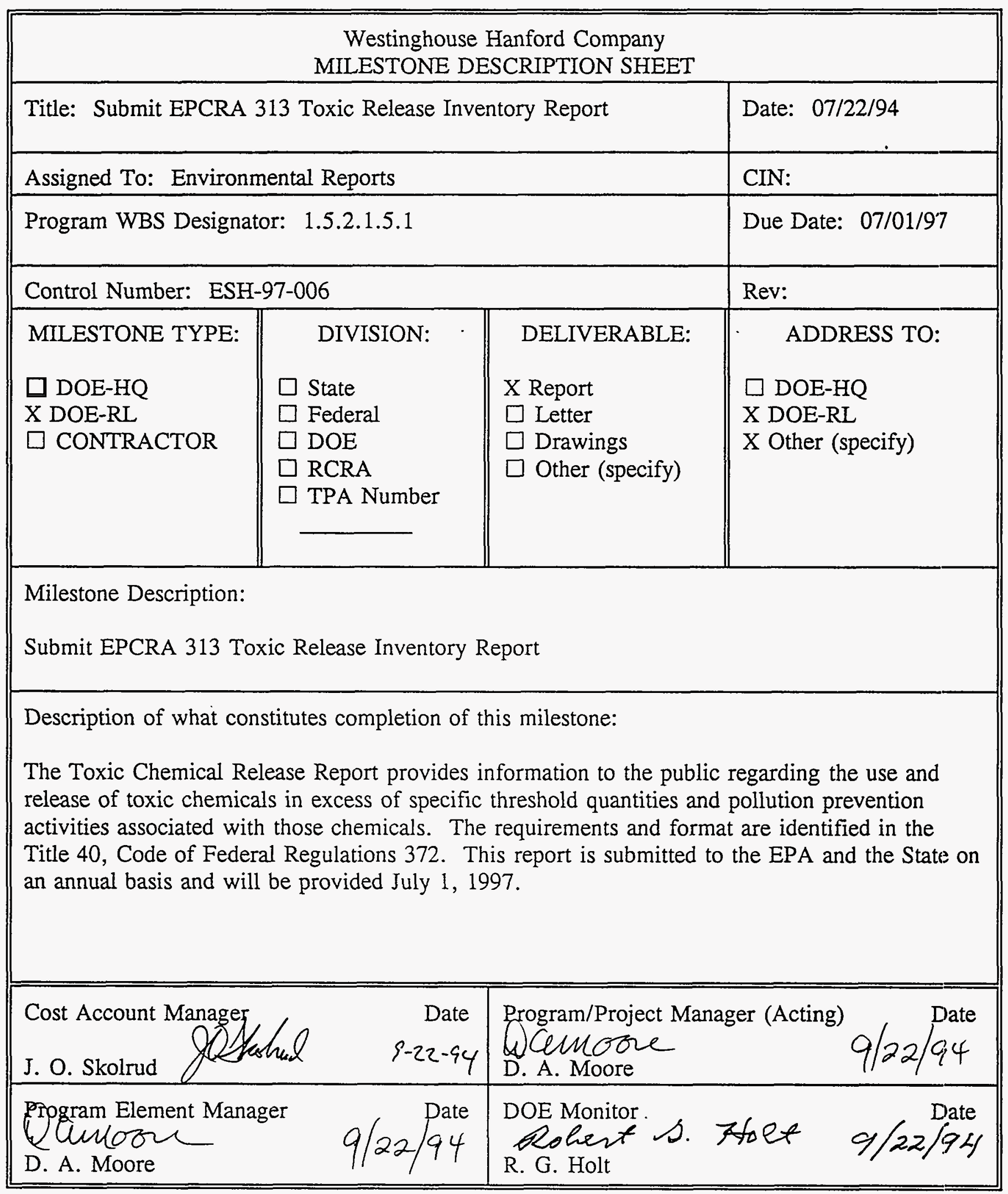




\begin{tabular}{|c|c|c|c|}
\hline \multicolumn{4}{|c|}{$\begin{array}{l}\text { Westinghouse Hanford Company } \\
\text { MILESTONE DESCRIPTION SHEET }\end{array}$} \\
\hline \multicolumn{3}{|c|}{$\begin{array}{l}\text { Title: Submit } 1996 \text { Hanford Site Annual Polychlorinated Biphenyl } \\
\text { (PCB) Document Log }\end{array}$} & Date: $07 / 22 / 94$ \\
\hline \multicolumn{3}{|c|}{ Assigned To: Environmental Reports } & CIN: \\
\hline \multicolumn{3}{|c|}{ Program WBS Designator: 1 -5.2.1.5.1 } & Due Date: $07 / 01 / 97$ \\
\hline \multicolumn{3}{|c|}{ Control Number: ESH-97-007 } & Rev: \\
\hline $\begin{array}{l}\text { MILESTONE TYPE: } \\
\square \text { DOE-HQ } \\
\text { X DOE-RL } \\
\square \text { CONTRACTOR }\end{array}$ & $\begin{array}{l}\quad \text { DIVISION: } \\
\square \text { State } \\
\square \text { Federal } \\
\square \text { DOE } \\
\square \text { RCRA } \\
\square \text { TPA Number }\end{array}$ & $\begin{array}{l}\text { DELIVERABLE } \\
\square \text { Report } \\
\text { X Letter } \\
\square \text { Drawings } \\
\square \text { Other (specify) }\end{array}$ & $\begin{array}{l}\quad \text { ADDRESS TO: } \\
\square \text { DOE-HQ } \\
\text { X DOE-RL } \\
\square \text { Other (specify) }\end{array}$ \\
\hline \multicolumn{4}{|c|}{$\begin{array}{l}\text { Milestone Description: } \\
\text { Submit } 1996 \text { Hanford Site Annual Polychlorinated Biphenyl (PCB) Document Log. }\end{array}$} \\
\hline \multicolumn{4}{|c|}{$\begin{array}{l}\text { Description of what constitutes completion of this milestone: } \\
\text { The } 1996 \text { PCB Annual Document Log provides information on the PCB waste handling activities } \\
\text { on the Hanford Site for calendar year. It is required to be prepared annually. This requirement } \\
\text { is contained in the Title } 40 \text {, Code of Federal Regulations } 761.180 \text {, Records and Monitoring. } \\
\text { The final } 1996 \text { PCB Document Log is to be provided to RL by July } 1,1997 \text {. }\end{array}$} \\
\hline \multicolumn{2}{|c|}{ Jost Account Manager O. Skolrud } & \multicolumn{2}{|c|}{$\begin{array}{l}\text { Brogram/Project Manager (Acting) } \\
\text { (4) Date } \\
\text { D. A. Moore }\end{array}$} \\
\hline \multicolumn{2}{|c|}{$\begin{array}{l}\text { Progkam Element Manager } \\
\text { (4) } 9 / 22 \\
\text { D. A. Moore }\end{array}$} & $\begin{array}{l}\text { DOE Monitor } \\
\text { Rolhect } \\
\text { R. G. Holt }\end{array}$ & Tto et $9 / 22 / 94$ \\
\hline
\end{tabular}




\begin{tabular}{|c|c|c|c|}
\hline \multicolumn{4}{|c|}{$\begin{array}{l}\text { Westinghouse Hanford Company } \\
\text { MILESTONE DESCRIPTION SHEET }\end{array}$} \\
\hline \multicolumn{3}{|c|}{ Title: Submit Annual Polycholorinated Biphenyl (PCB) Report } & Date: $07 / 22 / 94$ \\
\hline \multicolumn{3}{|c|}{ Assigned To: Environmental Reports } & CIN: \\
\hline \multicolumn{3}{|c|}{ Program WBS Designator: 1 -5.2.1.5.1 } & Due Date: $07 / 15 / 97$ \\
\hline \multicolumn{3}{|c|}{ Control Number: ESH-97-008 } & Rev: \\
\hline $\begin{array}{l}\text { MILESTONE TYPE: } \\
\square \text { DOE-HQ } \\
\text { X DOE-RL } \\
\square \text { CONTRACTOR }\end{array}$ & $\begin{aligned} & \text { DIVISION: } \\
& \\
& \text { State } \\
& \text { Federal } \\
& \square \text { DOE } \\
& \text { RCRA } \\
& \square \text { TPA Number }\end{aligned}$ & $\begin{array}{l}\text { DELIVERABLE: } \\
\text { X Report } \\
\square \text { Letter } \\
\square \text { Drawings } \\
\square \text { Other (specify) }\end{array}$ & $\begin{array}{l}\quad \text { ADDRESS TO: } \\
\square \text { DOE-HQ } \\
\text { X DOE-RL } \\
\text { X Other (specify) } \\
\text { Agencies }\end{array}$ \\
\hline \multicolumn{4}{|c|}{$\begin{array}{l}\text { Milestone Description: } \\
\text { Submit Annual Polycholorinated Biphenyl (PCB) Report }\end{array}$} \\
\hline \multicolumn{4}{|c|}{$\begin{array}{l}\text { Description of what constitutes completion of this milestone: } \\
\text { The } 1996 \text { PCB Annual Report provides information on the PCB waste handling activities for the } \\
\text { low-level burial grounds } 218-\mathrm{E}-12 \mathrm{~B} \text { Trench } 94 \text { on the Hanford Site. This report is prepared } \\
\text { annually as required by the Title } 40 \text {, Code of Federal Regulations } 761.180 \text {, Records and } \\
\text { Monitoring. The report shall be provided to RL for transmittal to the EPA by July } 15,1997 \text {. }\end{array}$} \\
\hline \multicolumn{2}{|c|}{ Jost Account Manager O. Skolrud } & \multicolumn{2}{|c|}{$\begin{array}{l}\text { Program/Project Manager (Acting) } \\
\text { Geucuce } \\
\text { D. A. Moore }\end{array}$} \\
\hline \multicolumn{2}{|c|}{$\begin{array}{l}\text { Program Element Manager. } \\
\text { O OMGore } \\
\text { D. A. Moore }\end{array}$} & \multicolumn{2}{|c|}{$\begin{array}{l}\text { DOE Monitor } \\
\text { Ro hent s. Tto et } 9 / 22 / 94 \\
\text { R. G. Holt }\end{array}$} \\
\hline
\end{tabular}




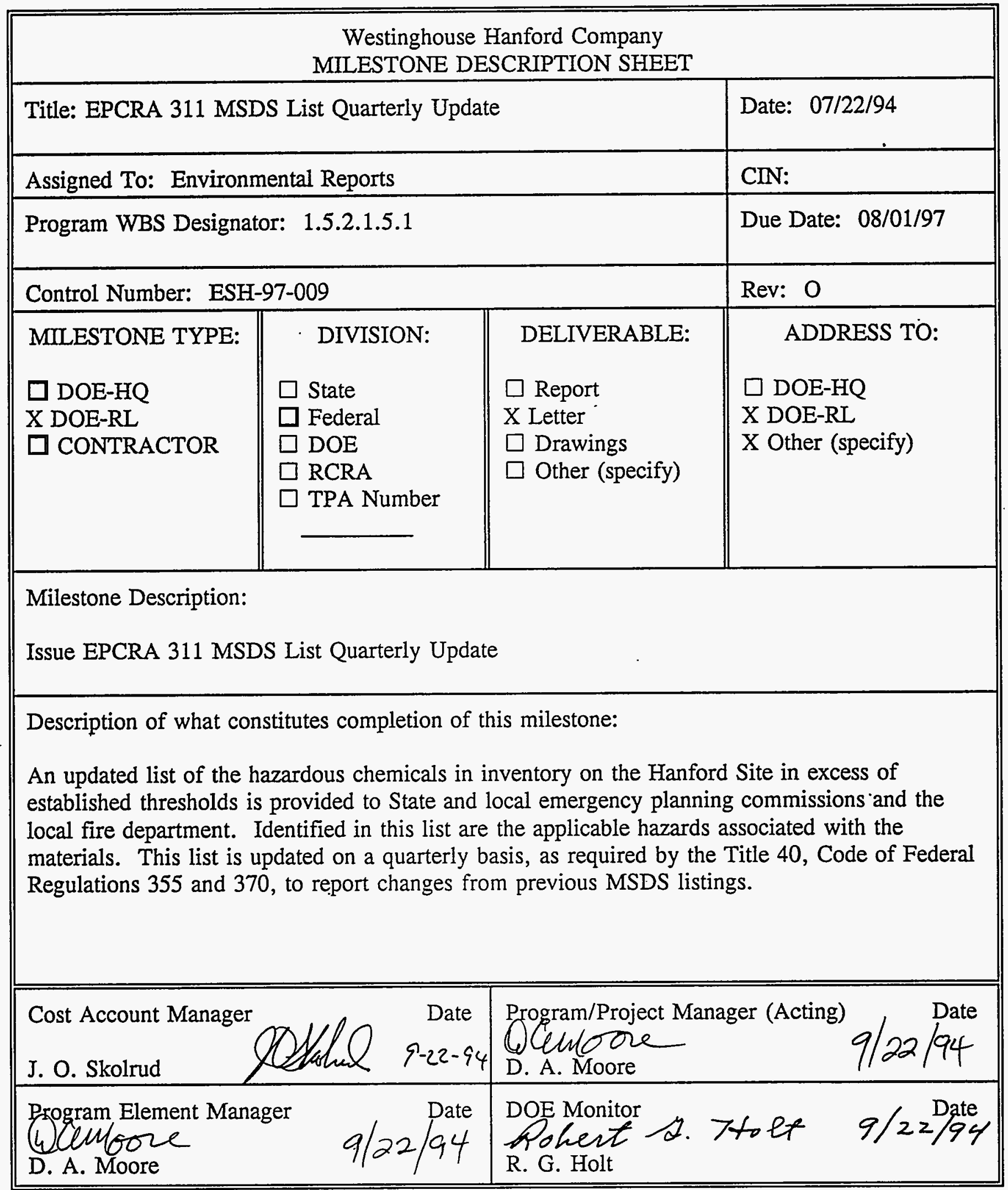


WHC-SP-1112

\begin{tabular}{|c|c|c|c|}
\hline \multicolumn{4}{|c|}{$\begin{array}{l}\text { Westinghouse Hanford Company } \\
\text { MILESTONE DESCRIPTION SHEET }\end{array}$} \\
\hline \multicolumn{3}{|c|}{ Title: Prepare Submittal to the National IDB } & Date: $07 / 22 / 94$ \\
\hline \multicolumn{3}{|c|}{ Assigned To: Environmental Reports } & CIN: \\
\hline \multicolumn{3}{|c|}{ Program WBS Designator: $1 \cdot 5 \cdot 2.1 .5 .1$} & Due Date: $08 / 29 / 97$ \\
\hline \multicolumn{3}{|c|}{ Control Number: ESH-97-010 } & Rev: \\
\hline $\begin{array}{l}\text { MILESTONE TYPE: } \\
\square \text { DOE-HQ } \\
\text { X DOE-RL } \\
\square \text { CONTRACTOR }\end{array}$ & $\begin{aligned} & \text { DIVISION: } \\
& \square \text { State } \\
& \text { Federal } \\
& \square \text { DOE } \\
& \text { RCRA } \\
& \text { TPA Number }\end{aligned}$ & $\begin{array}{l}\text { DELIVERABLE: } \\
\square \text { Report } \\
\text { X Letter } \\
\square \text { Drawings } \\
\text { X Other (specify) } \\
\text { Inventory Data }\end{array}$ & $\begin{array}{l}\quad \text { ADDRESS TO: } \\
\square \text { DOE-HQ } \\
\text { X DOE-RL } \\
\text { X Other (specify) } \\
\text { See below }\end{array}$ \\
\hline \multicolumn{4}{|c|}{ Milestone Description: } \\
\hline \multicolumn{4}{|c|}{$\begin{array}{l}\text { Description of what constitutes completion of this milestone: } \\
\text { This milestone consists of three data package letter reports; high-level, low-level, and TRU } \\
\text { waste status and projections. Data for each data package are prepared and sent from RL to Oak } \\
\text { Ridge through the respective technical support offices. The final product is a report on the } \\
\text { radioactive wastes in the USA and the anticipated volumes of new waste that will be generated } \\
\text { during the next } 35 \text { years. The report is used by DOE-HQ for strategic planning. }\end{array}$} \\
\hline \multicolumn{2}{|c|}{$\begin{array}{l}\text { Cost Account Manager } \\
\text { J. O. Skolrud }\end{array}$} & \multicolumn{2}{|c|}{$\begin{array}{l}\text { Program/Project Manager (Acting) } \\
\text { (4) Cutoore } \\
\text { D. A. Moore } 9 / 22 / 94\end{array}$} \\
\hline \multicolumn{2}{|c|}{$\begin{array}{l}\text { Program Element Manager } \\
\text { Auhoore } \\
\text { D. A. Moore }\end{array}$} & \multicolumn{2}{|c|}{$\begin{array}{l}\text { DOE Monitor } y \text {. Tto ef } 9 / 22 / 94 \\
\text { Polent } y \text { ate } \\
\text { R. G. Holt }\end{array}$} \\
\hline
\end{tabular}




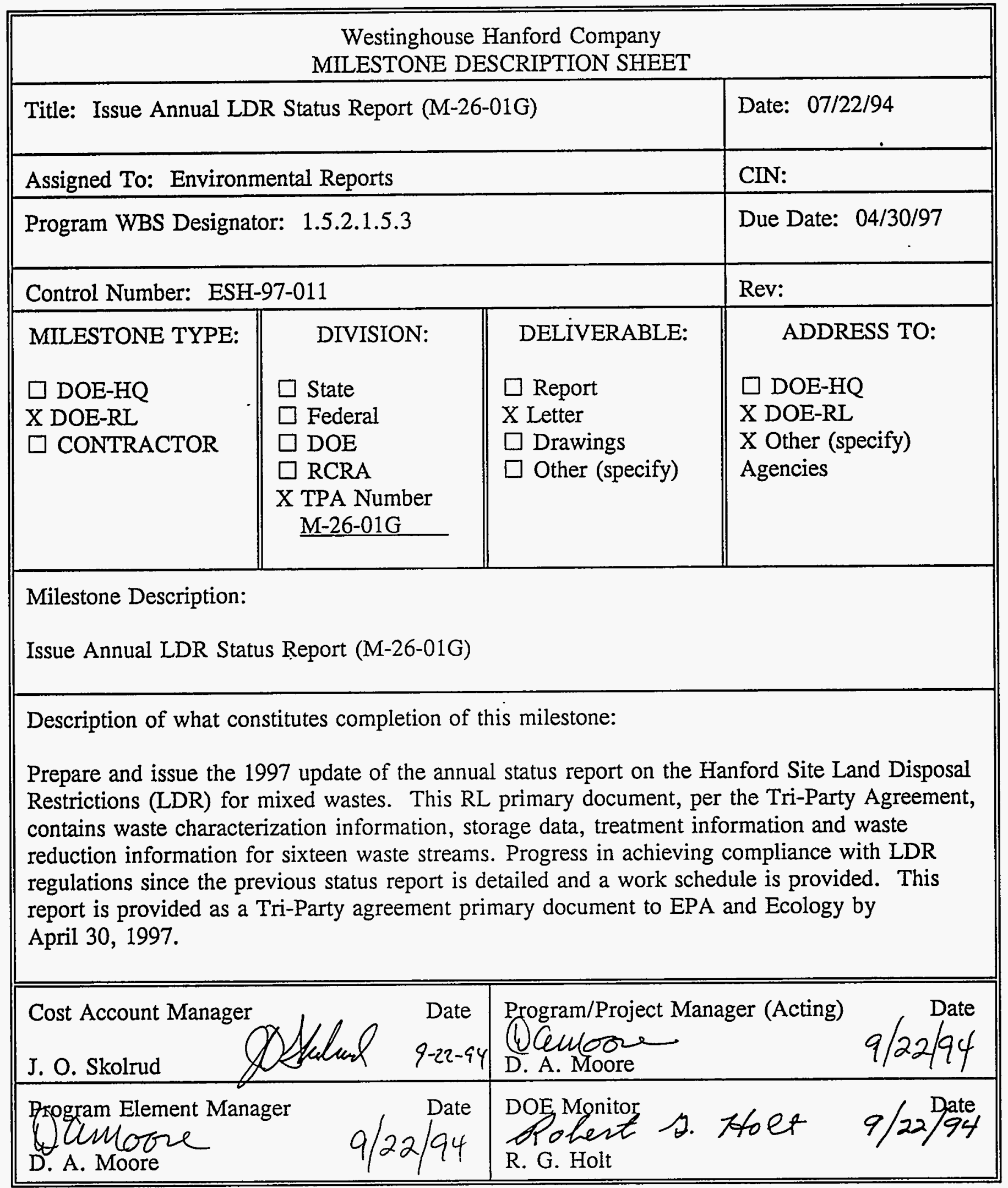




\begin{tabular}{|c|c|c|c|}
\hline \multicolumn{4}{|c|}{$\begin{array}{l}\text { Westinghouse Hanford Company } \\
\text { MILESTONE DESCRIPTION SHEET }\end{array}$} \\
\hline \multicolumn{3}{|c|}{ Title: Develop 3 Performance Measures/Indicators for P2 Program } & Date: $07 / 22 / 94$ \\
\hline \multicolumn{3}{|c|}{ Assigned To: Reengineering Program Office } & CIN: \\
\hline \multicolumn{3}{|c|}{ Program WBS Designator: 1.5.2.2.1.1 } & Due Date: $03 / 31 / 97$ \\
\hline \multicolumn{3}{|c|}{ Control Number: ESH-97-012 } & Rev: \\
\hline $\begin{array}{l}\text { MILESTONE } \\
\text { TYPE: } \\
\square \text { DOE-HQ } \\
\text { X DOE-RL } \\
\square \text { CONTRACTOR }\end{array}$ & $\begin{aligned} & \text { DIVISION: } \\
& \square \text { State } \\
\square & \text { Federal } \\
& \text { DOE } \\
\square & \text { RCRA } \\
\square & \text { TPA Number }\end{aligned}$ & $\begin{array}{l}\text { DELIVERABLE: } \\
\square \text { Report } \\
\square \text { Letter } \\
\square \text { Drawings } \\
\text { X Other (specify) }\end{array}$ & $\begin{array}{l}\text { ADDRESS TO: } \\
\square \text { DOE-HQ } \\
\text { X DOE-RL } \\
\square \text { Other (specify) }\end{array}$ \\
\hline \multicolumn{4}{|c|}{$\begin{array}{l}\text { Milestone Description: } \\
\text { Develop } 3 \text { Performance Measures/Indicators for P2 Program }\end{array}$} \\
\hline \multicolumn{4}{|c|}{$\begin{array}{l}\text { Description of what constitutes completion of this milestone: } \\
\text { Three performance measures/indicators submitted to RL by due date }\end{array}$} \\
\hline \multicolumn{2}{|c|}{ 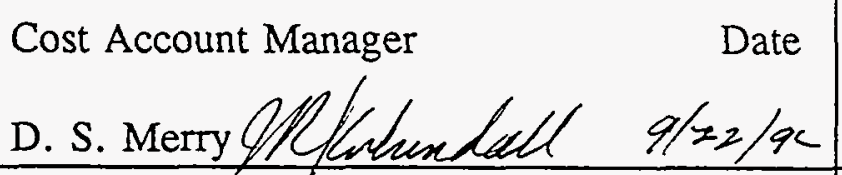 } & \multicolumn{2}{|c|}{$\begin{array}{l}\text { Program/Project Manager (Acting) } \\
\text { G) Qlemoore } \\
\text { D. A. Matere }\end{array}$} \\
\hline \multicolumn{2}{|c|}{$\begin{array}{l}\text { Program Element Manager } \\
\text { D. A. Moore }\end{array}$} & \multicolumn{2}{|c|}{ E. B. Dagan $A B$. Dagan $q \operatorname{lnq} q 4$} \\
\hline
\end{tabular}




\begin{tabular}{|c|c|c|c|}
\hline \multicolumn{4}{|c|}{$\begin{array}{l}\text { Westinghouse Hanford Company } \\
\text { MILESTONE DESCRIPTION SHEET }\end{array}$} \\
\hline \multicolumn{3}{|c|}{ Title: Prepare and Submit WAC 173-307 Annual Progress Report } & Date: $07 / 22 / 94$ \\
\hline \multicolumn{3}{|c|}{ Assigned To: Reengineering Program Office } & CIN: \\
\hline \multicolumn{3}{|c|}{ Program WBS Designator: 1 5.2.2.1.1 } & Due Date: $09 / 02 / 97$ \\
\hline \multicolumn{3}{|c|}{ Control Number: ESH-97-013 } & Rev: \\
\hline $\begin{array}{l}\text { MILESTONE TYPE: } \\
\square \text { DOE-HQ } \\
\text { X DOE-RL } \\
\square \text { CONTRACTOR }\end{array}$ & $\begin{aligned} & \text { DIVISION: } \\
& \\
& \text { State } \\
& \square \text { Federal } \\
& \text { DOE } \\
& \text { RCRA } \\
& \square \text { TPA Number }\end{aligned}$ & $\begin{array}{l}\text { DELIVERABLE: } \\
\text { X Report } \\
\square \text { Letter } \\
\square \text { Drawings } \\
\square \text { Other (specify) }\end{array}$ & $\begin{array}{l}\quad \text { ADDRESS TO: } \\
\square \text { DOE-HQ } \\
\text { X DOE-RL } \\
\square \text { Other (specify) }\end{array}$ \\
\hline \multicolumn{4}{|c|}{$\begin{array}{l}\text { Milestone Description: } \\
\text { Prepare and Submit WAC 173-307 Annual Progress Report }\end{array}$} \\
\hline \multicolumn{4}{|c|}{$\begin{array}{l}\text { Description of what constitutes completion of this milestone: } \\
\text { Annual progress report delivered go RL by due date }\end{array}$} \\
\hline \multicolumn{2}{|c|}{ 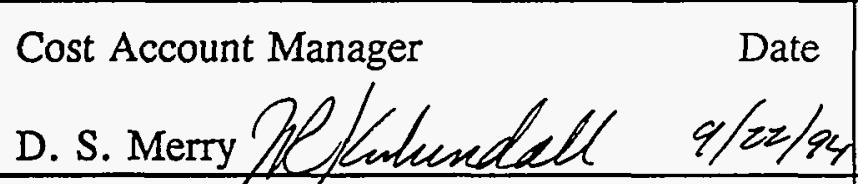 } & \multicolumn{2}{|c|}{$\begin{array}{ll}\text { Program/Project Manager (Acting) } & \text { Date } \\
\text { Damlare } & 9 / 22 / 94 \\
\text { D. A. Moore } & \end{array}$} \\
\hline \multicolumn{2}{|c|}{$\begin{array}{ll}\text { Pfogram Element Manager } \\
\text { De A. Mogze }\end{array}$} & \multicolumn{2}{|c|}{$\begin{array}{lr}\text { DOE Monitor } \\
\text { \&, }, \text {. Hagan } & \text { Date. } \\
\text { E. B. Dagan } & 9 / 22 / 94\end{array}$} \\
\hline
\end{tabular}




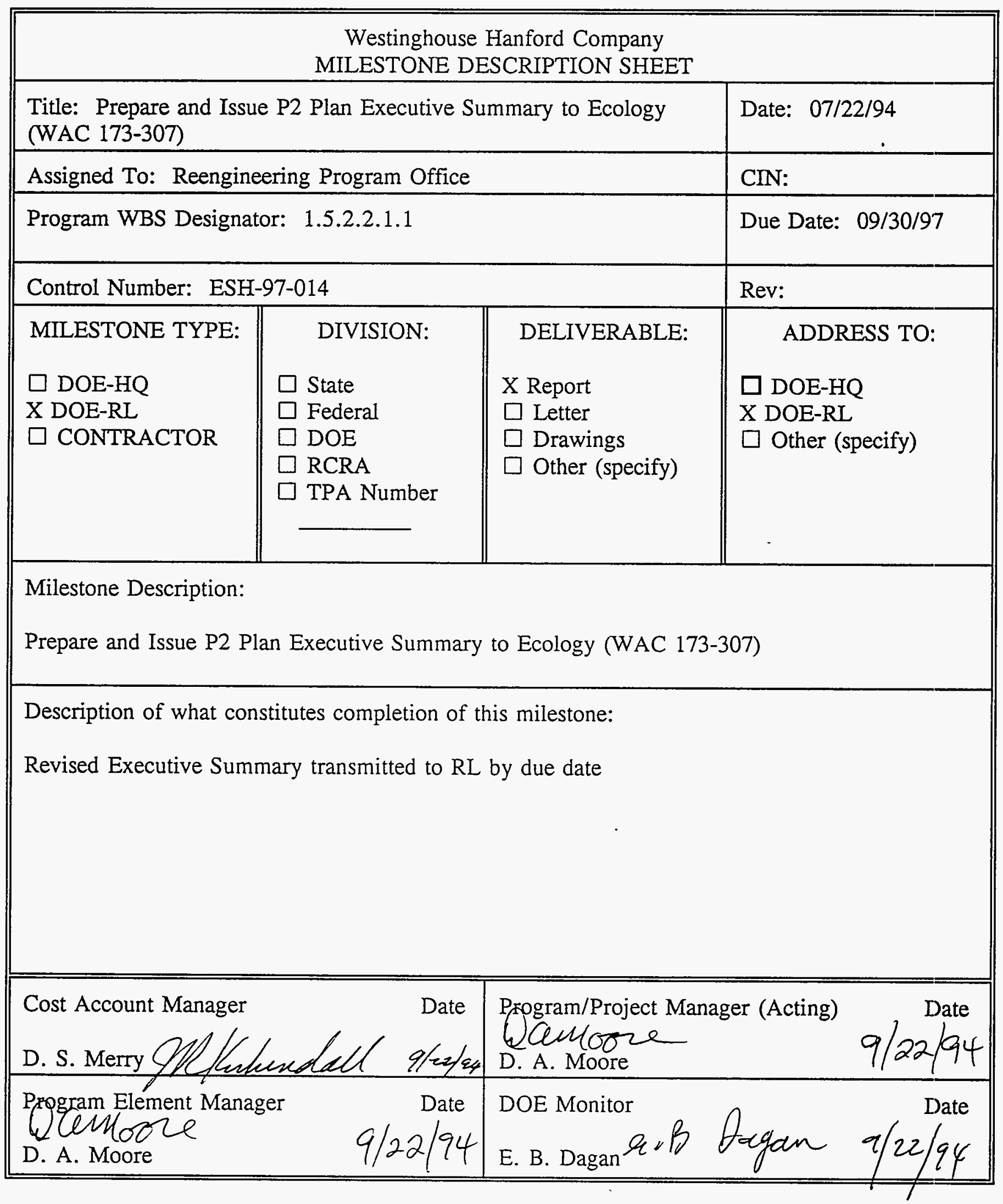




\begin{tabular}{|c|c|c|c|}
\hline \multicolumn{4}{|c|}{$\begin{array}{l}\text { Westinghouse Hanford Company } \\
\text { MILESTONE DESCRIPTION SHEET }\end{array}$} \\
\hline \multicolumn{3}{|c|}{$\begin{array}{l}\text { Title: Prepare Hanford Input to DOE Annual Report on Waste } \\
\text { Generation and Waste Minimization Progress (SEN-37-92) }\end{array}$} & Date: $07 / 22 / 94$ \\
\hline \multicolumn{3}{|c|}{ Assigned To: Reengineering Program Office } & CIN: \\
\hline \multicolumn{3}{|c|}{ Program WBS Designator: 1 -5.2.2.1.1 } & Due Date: $09 / 30 / 97$ \\
\hline \multicolumn{3}{|c|}{ Control Number: ESH-97-015 } & Rev: \\
\hline $\begin{array}{l}\text { MILESTONE TYPE: } \\
\text { X DOE-HQ } \\
\square \text { DOE-RL } \\
\square \text { CONTRACTOR }\end{array}$ & $\begin{aligned} & \text { DIVISION: } \\
& \\
& \text { State } \\
& \text { Federal } \\
& \text { DOE } \\
& \text { RCRA } \\
& \square \text { TPA Number }\end{aligned}$ & $\begin{array}{l}\text { DELIVERABLE: } \\
\text { X Report } \\
\square \text { Letter } \\
\square \text { Drawings } \\
\square \text { Other (specify) }\end{array}$ & $\begin{array}{l}\text { ADDRESS TO: } \\
\text { X DOE-HQ } \\
\square \text { DOE-RL } \\
\square \text { Other (specify) }\end{array}$ \\
\hline \multicolumn{4}{|c|}{$\begin{array}{l}\text { Milestone Description: } \\
\text { Prepare Hanford Input to DOE Annual Report on Waste Generation and Waste Minimization } \\
\text { Progress (SEN-37-92) }\end{array}$} \\
\hline \multicolumn{4}{|c|}{$\begin{array}{l}\text { Description of what constitutes completion of this milestone: } \\
\text { Hanford input submitted to RL by due date }\end{array}$} \\
\hline \multicolumn{2}{|c|}{ 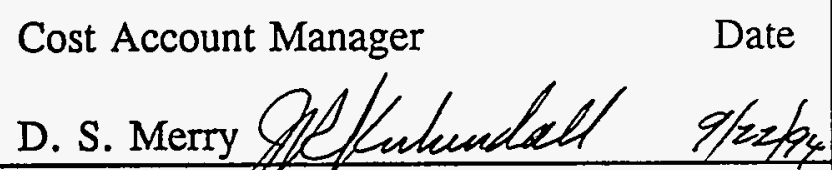 } & \multicolumn{2}{|c|}{$\begin{array}{l}\text { Program/Project Manager (Acting) } \\
\text { (C) } \\
\text { D. A. Moore }\end{array}$} \\
\hline \multicolumn{2}{|c|}{$\begin{array}{lc}\text { Prggram Element Manager } & \text { Date } \\
\text { W.inlooze } & 9 / 22 / 94 \\
\text { D. A. Moore } & 9 / 2\end{array}$} & \multicolumn{2}{|c|}{ D. B. Dagan $\dot{A}^{\text {DOE Monitor Dan } 9 / 22 / 94}$} \\
\hline
\end{tabular}




\begin{tabular}{|c|c|c|c|c|c|c|c|c|}
\hline \multicolumn{9}{|c|}{$2 \mathrm{H}$. Cost Baseline Summary ( $\$$ in Thousands) } \\
\hline $\begin{array}{c}\text { Program Element / } \\
\text { ADS \# }\end{array}$ & $\begin{array}{c}\text { Fund Type } \\
\text { (OP, LI, } \\
\text { CE, GPP, } \\
\text { ADS) }\end{array}$ & $\begin{array}{c}\text { FY } 1995 \text { SMS } \\
\text { Guidance }\end{array}$ & $\begin{array}{c}\text { FY } 1996 \\
\text { Target }\end{array}$ & $\begin{array}{c}\text { FY } 1997 \\
\text { Target }\end{array}$ & $\begin{array}{c}\text { FY } 1998 \\
\text { Target }\end{array}$ & $\begin{array}{c}\text { FY } 1999 \\
\text { Target }\end{array}$ & $\begin{array}{l}\text { FY } 2000 \\
\text { Target }\end{array}$ & $\begin{array}{l}\text { FY } 2001 \\
\text { Target }\end{array}$ \\
\hline \multirow[t]{2}{*}{ 1.5.2.1/7330-0 } & $O P$ & 8,489 & 8,445 & 10,357 & 11,042 & 9,842 & 10,401 & 10,714 \\
\hline & CE & 170 & 0 & 0 & 0 & 0 & 0 & 0 \\
\hline \multirow[t]{2}{*}{$1.5 .2 .2 / 7332-0$} & $O P$ & 599 & 566 & 583 & 883 & 970 & 867 & 893 \\
\hline & $\mathrm{CE}$ & 0 & 0 & 0 & 0 & 0 & 0 & 0 \\
\hline \multirow{2}{*}{$\begin{array}{l}\text { Total Program } \\
\text { Cost Summary }\end{array}$} & $\mathrm{OP}$ & 9,088 & 9,011 & 10,940 & 11,925 & 10,812 & 11,268 & 11,607 \\
\hline & CE & 170 & 0 & 0 & 0 & 0 & 0 & 0 \\
\hline \multicolumn{2}{|c|}{ Productivity Commitment } & -926 & -901 & -1641 & -2385 & -1081 & $-1,127$ & $-1,161$ \\
\hline \multicolumn{2}{|c|}{ Grand Total } & 8,332 & 8,110 & 9,299 & 9,540 & 9,731 & 10,141 & 10,446 \\
\hline
\end{tabular}

- Productivity Commitment $=10 \%$ in FY 1995, 10\% in FY 1996, 15\% in FY 1997, 20\% in FY 1998, 10\% in FY 1999, 10\% in FY 2000, $10 \%$ in FY 2001 


\section{Basis of Estimate}

Cost estimating forms were completed for all HEMP cost accounts and can be found in the 2440 Stevens Center Building in Room 2120 
Program:

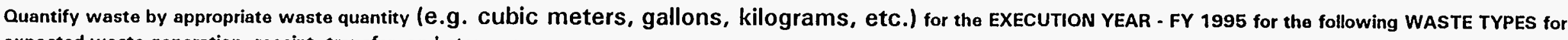
expected waste generation, receipt, transfer, and storage:

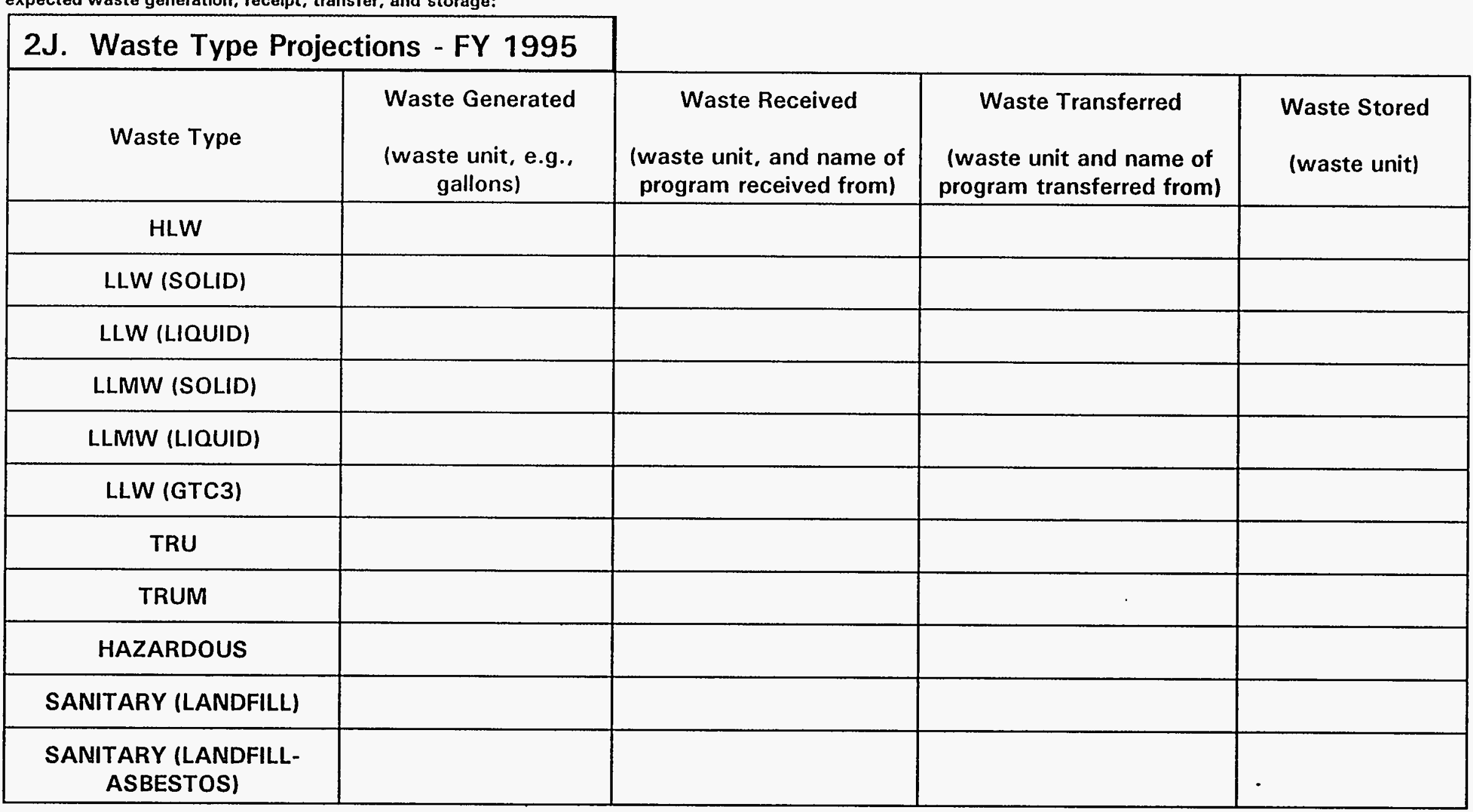


FY 1995 MYPP

HANFORD ENVIRONMENTAL MANAGEMENT PROGRAM

WHC-SP-1112

FOR THE EXECUTION YEAR - FY 1995 PROVIDE A BRIEF NARRATIVE FOR EACH OF THE FOLLOWING:

PLANS FOR WASTE STORAGE:

PLANS FOR INTERPROGRAM WASTE TRANSFER AND/OR DISPOSITION:

SUMMARY WASTE TYPE ISSUES: 
Program:

Quantify waste by appropriate waste quantity (e.g. Cubic meters, gallons, kilograms, etc.) for FY 1996 for the following WASTE TYPES for expected waste generation, receipt, transfer, and storage:

\begin{tabular}{|c|c|c|c|c|}
\hline 2J. Waste Type Projections - FY 1996 & \multicolumn{2}{|l|}{} \\
\hline Waste Type & $\begin{array}{c}\text { Waste Generated } \\
\text { (waste unit, e.g., } \\
\text { gallons) }\end{array}$ & $\begin{array}{c}\text { Waste Received } \\
\text { (waste unit, and name of } \\
\text { program received from) }\end{array}$ & $\begin{array}{c}\text { Waste Transferred } \\
\text { (waste unit and name of } \\
\text { program transferred from) }\end{array}$ & $\begin{array}{c}\text { Waste Stored } \\
\text { (waste unit) }\end{array}$ \\
\hline HLW & & & & \\
\hline LLW (SOLID) & & & & \\
\hline LLW (LIOUID) & & & & \\
\hline LLMW (SOLID) & & & & \\
\hline LLMW (LIQUID) & & & & \\
\hline LLW (GTC3) & & & & \\
\hline TRU & & & & \\
\hline TRUM & & & & \\
\hline HAZARDOUS & & & & \\
\hline SANITARY (LANDFILL) & & & & \\
\hline SANITARY (LANDFILL- \\
ASBESTOS) & & & & \\
\hline
\end{tabular}


FOR THE YEAR FY 1996, PROVIDE A BRIEF NARRATIVE FOR EACH OF THE FOLLOWING:

PLANS FOR WASTE STORAGE:

PLANS FOR INTERPROGRAM WASTE TRANSFER AND/OR DISPOSITION:

SUMMARY WASTE TYPE ISSUES: 
Program:

Quantify waste by appropriate waste quantity (e.g. Cubic meters, gallons, kilograms, etc.) for FY 1997 for the following WASTE TYPES for expected waste generation, receipt, transfer, and storage:

\begin{tabular}{|c|c|c|c|c|}
\hline \multicolumn{2}{|c|}{ 2J. Waste Type Projections - FY 1997} & \multirow[b]{2}{*}{$\begin{array}{c}\text { Waste Received } \\
\text { (waste unit, and'name of } \\
\text { program received from) }\end{array}$} & \multirow[b]{2}{*}{$\begin{array}{l}\text { Waste Transferred } \\
\text { (waste unit and name of } \\
\text { program transferred from) }\end{array}$} & \multirow[b]{2}{*}{$\begin{array}{l}\text { Waste Stored } \\
\text { (waste unit) }\end{array}$} \\
\hline Waste Type & $\begin{array}{l}\text { Waste Generated } \\
\text { (waste unit, e.g., } \\
\text { gallons) }\end{array}$ & & & \\
\hline \multicolumn{5}{|l|}{ HLW } \\
\hline \multicolumn{5}{|l|}{ LLW (SOLID) } \\
\hline \multicolumn{5}{|l|}{ LLW (LIQUID) } \\
\hline \multicolumn{5}{|l|}{ LLMW (SOLID) } \\
\hline \multicolumn{5}{|l|}{ LLMW (LIQUID) } \\
\hline \multicolumn{5}{|l|}{ LLW GTC3 } \\
\hline \multicolumn{5}{|l|}{ TRU } \\
\hline \multicolumn{5}{|l|}{ TRUM } \\
\hline \multicolumn{5}{|l|}{ HAZARDOUS } \\
\hline \multicolumn{5}{|l|}{ SANITARY (LANDFILL) } \\
\hline $\begin{array}{l}\text { SANITARY (LANDFILL- } \\
\text { ASBESTOS) }\end{array}$ & & & & \\
\hline
\end{tabular}


E
5
0
0
5
5
3

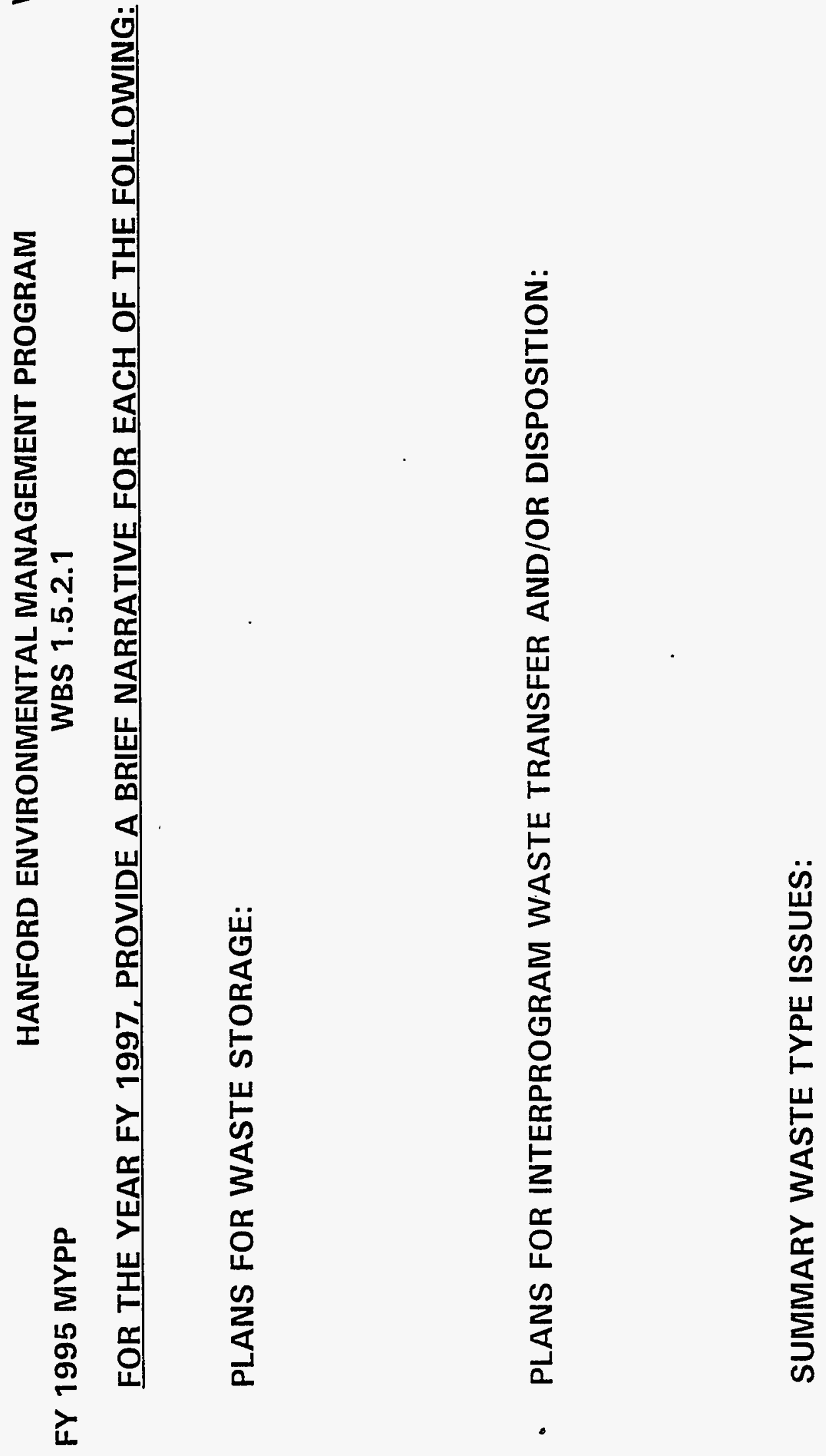


Program:

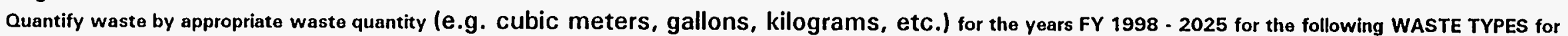
expected waste generation, receipt, transfer, and storage:

\begin{tabular}{|c|c|c|c|c|}
\hline 2J. Waste Type Projections - FY 1998 - 2025 & & & \\
\hline Waste Type & $\begin{array}{c}\text { Waste Generated } \\
\text { (waste unit, e.g., } \\
\text { gallons) }\end{array}$ & $\begin{array}{c}\text { Waste Received } \\
\text { (waste unit, and name of } \\
\text { program received from) }\end{array}$ & $\begin{array}{c}\text { Waste Transferred } \\
\text { (waste unit and name of } \\
\text { program transferred from) }\end{array}$ & $\begin{array}{c}\text { Waste Stored } \\
\text { (waste unit) }\end{array}$ \\
\hline HLW & & & & \\
\hline LLW (SOLID) & & & & \\
\hline LLW (LIOUID) & & & & \\
\hline LLMW (SOLID) & & & & \\
\hline LLMW (LIQUID) & & & & \\
\hline LLW GTC3 & & & & \\
\hline TRU & & & & \\
\hline TRUM & & & & \\
\hline HAZARDOUS & & & & \\
\hline SANITARY (LANDFILL) & & & & \\
\hline SANITARY (LANDFILL- \\
ASBESTOS)
\end{tabular}




\section{WBS 1.5.2.1}

\begin{tabular}{|c|c|c|c|c|c|c|c|}
\hline 2K. Planned Staffing (Full-Time Eq & aalents) & & & & Job Fam & ily only & fter 1997 \\
\hline JOB FAMILY & & & & & & & \\
\hline Job category & 1995 & 1996 & 1997 & 1998 & 1999 & 2000 & 2001 \\
\hline MANAGERS & 12,5 & 12.5 & $12 \div 5$ & 12.5 & $\because 2,5$ & $12 \cdot 5$ & $12: 5$ \\
\hline First line & 2.7 & 2.7 & & & & & \\
\hline Manager \& Executive & 9.3 & 9.3 & & & & & \\
\hline Program & 0.5 & 0.5 & & & & & \\
\hline ENGINEERS & 28.1 & 28.1 & 28.1 & 28.1 & 281 & 28.1 & 28.1 \\
\hline Chemical & 7.5 & 7.5 & & & & & \\
\hline Civil & 1.4 & 1.4 & & & & & \\
\hline Computer & 0.2 & 0.2 & & & & & \\
\hline Environmental & 7.1 & 7.1 & & & & & \\
\hline Mechanical & 2.0 & 2.0 & & & & & \\
\hline Mining & 2.0 & 2.0 & & & & & \\
\hline Plant & 4.7 & 4.7 & & & & & \\
\hline Safety & 1.9 & 1.9 & & & & & \\
\hline Other & 1.3 & 1.3 & & & & & \\
\hline SCIENTISTS & 6.6 & 6.6 & 6.6 & 66 & 6.6 & $\%+6.6$ & $\therefore 6.6$ \\
\hline Chemists & 1.0 & 1.0 & & & & & \\
\hline Environmental & 4.6 & 4.6 & & & & & \\
\hline Life & 1.0 & 1.0 & & & & . & \\
\hline
\end{tabular}


WBS 1.5.2.1

\begin{tabular}{|c|c|c|c|c|c|c|c|}
\hline \multicolumn{5}{|c|}{ 2K. Planned Staffing (Full-Time Equivalents) } & \multicolumn{3}{|c|}{ Job Family Only After 1997} \\
\hline \multicolumn{8}{|l|}{ JOB FAMILY } \\
\hline Job category & 1995 & 1996 & 1997 & 1998 & 1999 & 2000 & 2001 \\
\hline ADMIN/OTHER PROFESSIONALS & 10.3 & 10.3 & 10.3 & 10.3 & 10.3 & 10.3 & 10.3 \\
\hline Accountants & 1.5 & 1.5 & & & & & \\
\hline Computer System Analyst & 4.0 & 4.0 & & & & & \\
\hline Scheduler & 4.0 & 4.0 & & & & & \\
\hline Trainers \& Instructors & 0.5 & 0.5 & & & & & \\
\hline Tech Writers \& Editors & 0.3 & 0.3 & & & & & \\
\hline GEN ADM/SECRETARY/CLERK & 17.5 & 17.5 & 17.5 & 17.5 & 17.5 & 17.5 & 17,5 \\
\hline Office Clerks (Gen) & 10.3 & 10.3 & & & & & \\
\hline Secretaries & 7.2 & 7.2 & & & & & \\
\hline \multicolumn{8}{|c|}{ TOTALS BY CATEGORIES } \\
\hline EXEMPT & 57.5 & 57.5 & & & & & \\
\hline NON-EXEMPT NON BARGAINING & 17.5 & 17.5 & & & & & \\
\hline GRAND TOTALS & 75.0 & 75.0 & 75.0 & 75.0 & 75.0 & 75.0 & 75.0 \\
\hline
\end{tabular}


$\frac{N}{5}$
$\frac{1}{5}$
$\frac{0}{3}$

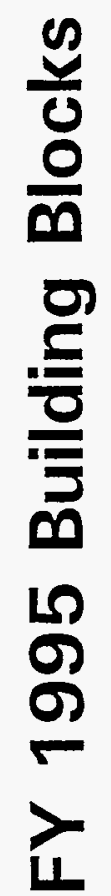


WBS 1.5.2.1

\begin{tabular}{|c|c|c|c|c|c|}
\hline Description (ADS) & $\begin{array}{c}\text { Program } \\
\text { Priority }\end{array}$ & $\begin{array}{l}\text { TPA } \\
\text { MS \# }\end{array}$ & $\begin{array}{l}\text { BCWs } \\
\text { Est. }\end{array}$ & $\begin{array}{l}\text { CUM } \\
\text { TOTAL }\end{array}$ & Comments \\
\hline $\begin{array}{l}\text { Program Administration (7330) } \\
\text {-1R1H11 - Program Administration } \\
\text { **Program Control } \\
\text { * Program Administration } \\
\end{array}$ & $B 1,35,2$ & & 334 & 334 & \\
\hline $\begin{array}{l}\text { Program Administration (7330) } \\
\text {-1R1H12 - Productivity Commitment }\end{array}$ & B1.35.2 & & (926) & .592 & $\begin{array}{l}\text { *NOTE: Any reduction to the Baseline will result in a } \\
\text { reduction to the calculated cost of the } 10 \% \\
\text { productivity commitment. }\end{array}$ \\
\hline $\begin{array}{l}\text { Environmental Reporting }(7330) \\
\text { *1R1H51 - Environmental Reports } \\
\text { "*Regulatory Reporting Management/Coordination } \\
\text { " EPCRA Report } \\
\text { "*Dangerous Waste Reporting } \\
\text { " *TSCA/FIFRA Reporting } \\
\text { " *Air Emissions Reporting } \\
\text { "*IDB Submittal } \\
\text { - "Site Wide Spill Reporting }\end{array}$ & $B 1,35,2$ & & 1759 & 1,167 & \\
\hline $\begin{array}{l}\text { Capital Equipment (7330) } \\
\text { "2R1H52 - CENRTC UNIX Computer Procurement (HMID II) }\end{array}$ & $81,35,2$ & & 131 & 1,298 & \\
\hline $\begin{array}{l}\text { Environmental Reporting }(7330) \\
\text { "1R1H53 - LDR Waste Status Reporting } \\
\text { " "Support Tri-Party Agreement Milestone }\end{array}$ & $B 1,35,2$ & $M-26$ & 140 & 1,438 & \\
\hline $\begin{array}{l}\text { Pollution Prevention (7332) } \\
\text {-1R1H81 - Pollution Prevention } \\
\qquad * \text { General Site Support }\end{array}$ & $B 1,35,2$ & & 599 & 2,037 & \\
\hline $\begin{array}{l}\text { RCRA Compliance (7330) } \\
\text { "1R1H78-RCRA Permitting Coordination } \\
\text { * General RCRA Permitting Coordination } \\
\text { " "Support Tri-Party Agreement Milestone } \\
\text { * Provide State/Federal Regulatory Analysis Support (previously } \\
\text { funded G\&A }\end{array}$ & $81,35,2$ & $M-20$ & 450 & 2.487 & $\cdot$ \\
\hline
\end{tabular}


WBS 1.5.2.1

\begin{tabular}{|c|c|c|c|c|c|}
\hline Description (ADS) & $\begin{array}{l}\text { Program } \\
\text { Priority }\end{array}$ & $\begin{array}{l}\text { TPA } \\
\text { MS \# }\end{array}$ & $\begin{array}{l}\text { BCWS } \\
\text { Est. }\end{array}$ & $\begin{array}{l}\text { CUM } \\
\text { TOTAL }\end{array}$ & Comments \\
\hline $\begin{array}{l}\text { RCRA Compliance }(7330) \\
\text { *1R1H71-RCRA Permit Compliance/Modification } \\
\text { *RCRA Permit Configuration Control } \\
\text { "*RCRA Permit Modifications } \\
\text { * Regulator Report Certifications } \\
\text { * Compliance Overview of Existing Systems } \\
\end{array}$ & B1,35,2 & & 1329 & 3,816 & \\
\hline $\begin{array}{l}\text { RCRA Compliance }(7330) \\
\text { *1R1H72 - RCRA Permit Condition Tracking } \\
\text { " "Computer database } \\
\text { * Individual TSD Unit compliance checklist } \\
\text { * TSD Unit trending of inspection and self-assessment results } \\
\text { * "Tickler" for due dates and new information identification }\end{array}$ & $B 1,35,2$ & & 544 & 4,360 & \\
\hline $\begin{array}{l}\text { RCRA Compliance (7330) } \\
\text { *1R1H73 - RCRA Permit Recordkeeping and Retrieval } \\
\text { * Record Location/ldentification Index (computerized database) } \\
\text { * TSD Unit listing of retained record requirements } \\
\text { * TSD Unit listing of documents in its Administrative Record } \\
\text { * Coordination of information for the Public Information Repositories }\end{array}$ & B1,35,2 & & 161 & 4,521 & \\
\hline $\begin{array}{l}\text { RCRA Compliance (7330) } \\
\text { "1R1H75 - Annual Closure Cost Estimate and Waste Minimization } \\
\text { Reports } \\
\text { " "Closure cost estimates are only needed for "final status" TSD } \\
\text { Units } \\
\text { " Waste Minimization Report includes all final status TSD Units }\end{array}$ & B1,35,2 & & 198 & 4,719 & \\
\hline $\begin{array}{l}\text { RCRA Compliance }(7330) \\
\text { "1R1H76 - RCRA Permits Waste Inventory } \\
\text { " Waste location, volume, and concentration by TSD Unit } \\
\text { * "Incorporate changes to the WIDS and SWMU waste information }\end{array}$ & B1,35,2 & & 54 & 4.773 & • \\
\hline
\end{tabular}




\section{L. Building Blocks}

FY 1995

\begin{tabular}{|c|c|c|c|c|c|}
\hline Description (ADS) & $\begin{array}{l}\text { Program } \\
\text { Priority }\end{array}$ & $\begin{array}{l}\text { TPA } \\
\text { MS \# }\end{array}$ & $\begin{array}{l}\text { BCWS } \\
\text { Est. }\end{array}$ & $\begin{array}{l}\text { CUM } \\
\text { TOTAL. }\end{array}$ & Comments \\
\hline $\begin{array}{l}\text { RCRA Compliance }(7330) \\
\text { *1R1H77 - Facility Wide Inspections } \\
\text { * Expands the existing TSD Unit-regulator inspection process } \\
\text { * Incorporates an inspection response process }\end{array}$ & B1,35,2 & & 151 & 4.924 & \\
\hline $\begin{array}{l}\text { Air and Water Permit Requirements (7330) } \\
\text { *1R1H41-Air and Water Permitting Coordination } \\
\text { * * General Air/Water Permitting Coordination } \\
\text { * Provide State/Federal Regulatory Analysis Support (previously } \\
\text { funded G\&A) } \\
\text { * RL Support for Wastewater Permits } \\
\text { * *Stormwtr/UIC/Drinking Water Permit } \\
\text { * Air Emission Inventories } \\
\text { - Air Operating Permit Application } \\
\text { * *FF-01, NESHAP and Portable Permit Support }\end{array}$ & B1,35,2 & & 1,109 & 6,033 & \\
\hline $\begin{array}{l}\text { Environmental Field Services }(7330) \\
\text {-1R1H31 - Environmental Field Services } \\
\text { * "Provide support for Facility Support Management (previously } \\
\text { funded by G\&A) } \\
\text { *"Escort regulatory agency inspectors onsite (previously funded } \\
\text { G\&A) } \\
\text { **Inspection Support } \\
\text { **Environmental Compliance } \\
\text { - Regulatory Compliance File Development }\end{array}$ & B1,35,2 & $M-32$ & 751 & 6,784 & \\
\hline $\begin{array}{l}\text { NEPA Coordination }(7330) \\
\text { *1R1H21 - NEPA Coordination } \\
\text { * NEPA Site Coordination } \\
\end{array}$ & B1,35,2 & & 202 & 6,986 & \\
\hline $\begin{array}{l}\text { Tri-Party Agreement Integration (7330) } \\
\text {-1R1H61-Tri-Party Agreement Integration } \\
\text { * Tri-Party Agreement Coordination/Business Integration Support } \\
\text { * Tri-Party Agreement Tactical Management } \\
\text { " Tri-Party Agreement Handbook Support } \\
\text { * Environmental Tracking System Support }\end{array}$ & B1,35,2 & & 1034 & 8,020 & - \\
\hline
\end{tabular}




\begin{tabular}{|c|c|c|c|c|c|}
\hline Description (ADS) & $\begin{array}{c}\text { Program } \\
\text { Priority }\end{array}$ & $\begin{array}{l}\text { TPA } \\
\text { MS \# }\end{array}$ & $\begin{array}{l}\text { BCWS } \\
\text { Est. }\end{array}$ & $\begin{array}{l}\text { CUM } \\
\text { TOTAL }\end{array}$ & Comments \\
\hline $\begin{array}{l}\text { Program Administration (7330) } \\
\text { "1R1H11 - Program Administration } \\
\text { * * Program Administration - Scheduler }\end{array}$ & B1,35,2 & & 96 & 8,116 & $\begin{array}{l}\text { (1) Identified for possible reduction (6/18 RB Agee } \\
\text { exercise) } \\
\text { *Failure to provide support to scheduling } \\
\text { activities (preparation of resource loaded } \\
\text { schedules) will result in non-compliance of } \\
\text { baseline validation criteria (scope, schedule, and } \\
\text { cost) }\end{array}$ \\
\hline $\begin{array}{l}\text { Tri-Party Agreement Integration (7330) } \\
\text { *1R1H61 - Tri-Party Agreement Integration } \\
\text { * *TWRS Milestone Manager }\end{array}$ & B1.35.2 & & 91 & 8,207 & $\begin{array}{l}\text { (2) Identified for possible reduction (6/20 RB Agee } \\
\text { exercise) } \\
\text { "Failure to provide adequate support will impact } \\
\text { coordination of Tri-Party Agreement } \\
\text { implementation and negotiations with regulators. }\end{array}$ \\
\hline $\begin{array}{l}\text { Program Administration }(7330) \\
{ }^{2} \mathrm{R} 1 \mathrm{H} 13 \text { - CENRTC Holding Account }\end{array}$ & B1,35,2 & & 39 & 8.246 & $\begin{array}{l}\text { (1) Identified for possible reduction (6/18 RB Agee } \\
\text { exercise) } \\
\text { *NO IMPACT }\end{array}$ \\
\hline $\begin{array}{l}\text { Tri-Party Agreement Integration (7330) } \\
\text { *1R1H61- Tri-Party Agreement Integration } \\
\text { * TWRS Milestone Manager }\end{array}$ & $B 1,35,2$ & & 86 & 8,332 & $\begin{array}{l}\text { (3) Identified for possible reduction (8/4 RL Janis } \\
\text { Ward exercise) } \\
\text { "Failure to provide adequate support will impact } \\
\text { coordination of Tri-Party Agreement } \\
\text { implementation and negotiations with regulators. }\end{array}$ \\
\hline $\begin{array}{l}\text { FY95 TOTAL PIT PROPOSED TARGET LESS } \\
10 \% \text { PRODUCTIVITY CHALLENGE }\end{array}$ & & & 8,332 & & \\
\hline Budget Adjustment & $B 1,35,2$ & & 926 & 9,258 & \\
\hline FY95 TOTAL PIT PROPOSED TARGET & & & 9,258 & & \\
\hline
\end{tabular}




\begin{tabular}{|c|c|c|c|c|c|}
\hline Description (ADS) & $\begin{array}{l}\text { Program } \\
\text { Priority }\end{array}$ & $\begin{array}{l}\text { TPA } \\
\text { MS \# }\end{array}$ & $\begin{array}{l}\text { BCWS } \\
\text { Est. }\end{array}$ & $\begin{array}{l}\text { CUM } \\
\text { TOTAL }\end{array}$ & Comments \\
\hline $\begin{array}{l}\text { Pollution Prevention (7332) } \\
\text { "1R1H81 - Pollution Prevention (P2) } \\
\text { * "FY94 Deferred Workscope - CY } 1993 \text { WG \& WM Progress Report } \\
\text { " Hazardous Materiais Reduction Act. } \\
\text { " Employee Involvement- Training } \\
\text { - Technical Assistance }\end{array}$ & $B 2,35,2$ & & 579 & 9.837 & \\
\hline $\begin{array}{l}\text { RCRA Compliance (7330) } \\
\text { *1R1H74 - Pipeline Mapping and Marking } \\
\text { "FY94 Deferred Workscope - Pipeline Mapping/Marking cost } \\
\text { estimation } \\
\text { *FY94 Deferred Workscope - Pipeline Mapping/Marking } \\
\text { Implementation Plan }\end{array}$ & $B 1,35,2$ & & 227 & 10,064 & \\
\hline FY95 TOTAL OMB BUDGET & & & 10,064 & & \\
\hline $\begin{array}{l}\text { Air and Water Permit Requirements }(7330) \\
\cdot 1 \mathrm{R} 1 \mathrm{H} 41 \text { - Air and Water Permitting Coordination } \\
\cdot * \text { Air Operating Permit Application Production Requirements }\end{array}$ & B1,35,2 & & 200 & 10,264 & \\
\hline $\begin{array}{l}\text { Environmental Reporting }(7330) \\
\text { " } 1 \text { R1H51 - Environmental Reports } \\
\text { *FY94 Deferred Workscope - Waste Data Management Systems } \\
\text { delayed pending new reporting requirements }\end{array}$ & B1,35,2 & & 42 & 10,306 & \\
\hline $\begin{array}{l}\text { NEPA Coordination (7330) } \\
\text {-1R1H22 - NRDA Coordination } \\
\text { * *FY94 Deferred Workscope - Facilitator }\end{array}$ & B1,35,2 & & 100 & 10,406 & $\begin{array}{l}\text { NOTE: Scope has been transferred to Bechtel. } \\
\text { Should the HEMP be funding this activity? }\end{array}$ \\
\hline $\begin{array}{l}\text { Pollution Prevention (7332) } \\
\text {-1R1H81 - Pollution Prevention (P2) } \\
\text { * Employee Involvement - Awareness } \\
\text { *P2 accomplishments database automation } \\
\text { * Information \& Technology Exchange } \\
\text { - Program Evaluation }\end{array}$ & $B 2,35,2$ & & 545 & 10,951 & - \\
\hline
\end{tabular}




\begin{tabular}{|c|c|c|c|c|c|}
\hline 2L. Building Blocks & FY 1995 & \multicolumn{3}{|c|}{ comments } \\
\hline Description (ADS) & $\begin{array}{l}\text { Program } \\
\text { Priority }\end{array}$ & $\begin{array}{c}\text { PPA } \\
\text { MS \# }\end{array}$ & $\begin{array}{c}\text { BCWs } \\
\text { Est. }\end{array}$ & $\begin{array}{c}\text { CUM } \\
\text { TOTAL }\end{array}$ & \\
\hline FY95 PLANNED BUDGET & & & 10,951 & & \\
\hline
\end{tabular}


$\frac{N}{5}$

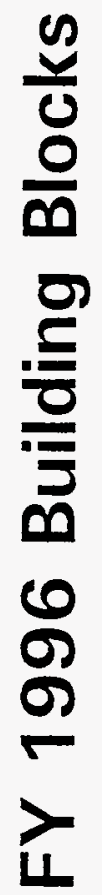




\begin{tabular}{|c|c|c|c|c|c|}
\hline Description (ADS) & $\begin{array}{l}\text { Program } \\
\text { Prioirty }\end{array}$ & $\begin{array}{c}\text { TPA } \\
\text { MS \# }\end{array}$ & $\begin{array}{c}\text { BCWS } \\
\text { Est. }\end{array}$ & $\begin{array}{l}\text { CUM } \\
\text { TOTAL. }\end{array}$ & Comments \\
\hline $\begin{array}{l}\text { Program Administration (7330) } \\
\text {-1R1H11 - Program Administration } \\
\bullet * \text { Program Control } \\
\bullet * \text { Program Administration }\end{array}$ & B1,35,2 & & 460 & 460 & \\
\hline $\begin{array}{l}\text { Program Administration (7330) } \\
\text { 1R1H12 - Productivity Commitment } \\
\bullet \text { *Program Adjustment }\end{array}$ & B1,35,2 & & (941) & -481 & $\begin{array}{l}\text { *NOTE: Any reduction to the Baseline will } \\
\text { result in a reduction to the calculated cost of } \\
\text { the } 10 \% \text { productivity commitment. }\end{array}$ \\
\hline $\begin{array}{l}\text { Environmental Reporting (7330) } \\
\text { 1R1H51 - Environmental Reports } \\
\text { " Regulatory Reporting Management/Coordination } \\
\text { "EPCRA Report } \\
\text { * Dangerous Waste Reporting } \\
\text { * TSCA/FIFRA Reporting } \\
\text { *Air Emissions Reporting } \\
\text { *IDB Submittal } \\
\text { * *Site Wide Spill Reporting }\end{array}$ & B1,35,2 & & 1.733 & 1,252 & \\
\hline $\begin{array}{l}\text { Environmental Reporting (7330) } \\
\text { "1R1H53 - LDR Waste Status Reporting } \\
\text { * "Support Tri-Party Agreement Milestone }\end{array}$ & B1,35,2 & $M-26$ & 149 & 1.401 & \\
\hline $\begin{array}{l}\text { Pollution Prevention (7332) } \\
\text {-1R1H81 - Pollution Prevention } \\
\text { * General Site Support }\end{array}$ & B1,35,2 & & 566 & 1,967 & \\
\hline $\begin{array}{l}\text { RCRA Compliance (7330) } \\
\text { * IR1H78 - RCRA Permitting Coordination } \\
\text { * General RCRA Permitting Coordination } \\
\text { * * Support Tri-Party Agreement Milestone } \\
\text { - Provide State and Federal Regulatory Analysis Support }\end{array}$ & B1,35,2 & M-20 & 393 & 2,360 & - \\
\hline
\end{tabular}


WBS 1.5.2.1

\begin{tabular}{|c|c|c|c|c|c|}
\hline Description (ADS) & $\begin{array}{l}\text { Program } \\
\text { Prioirty }\end{array}$ & $\begin{array}{l}\text { TPA } \\
\text { MS \# }\end{array}$ & $\begin{array}{c}\text { BCWs } \\
\text { Est. }\end{array}$ & $\begin{array}{l}\text { CUM } \\
\text { TOTAL }\end{array}$ & Comments \\
\hline $\begin{array}{l}\text { RCRA Compliance (7330) } \\
\text { "1R1H71 - RCRA Permit Compliance/Modification } \\
\text { " *RCRA Permit Configuration Control } \\
\text { " *RCRA Permit Modifications } \\
\text { " Regulator Report Certifications } \\
\text { " "Compliance Overview of Existing Systems }\end{array}$ & B1,35,2 & & 1257 & 3,617 & \\
\hline $\begin{array}{l}\text { RCRA Compliance }(7330) \\
\text { "1R1H72 - RCRA Permit Condition Tracking } \\
\text { " Computer database } \\
\text { " "Individual TSD Unit compliance checklist } \\
\text { " TSD Unit trending of inspection and self-assessment results } \\
\text { * "Tickler" for due dates and new information identification }\end{array}$ & B1,35,2 & & 572 & 4.189 & \\
\hline $\begin{array}{l}\text { RCRA Compliance (7330) } \\
\text { *1R1H73 - RCRA Permit Recordkeeping and Retrieval } \\
\text { * Record Location/ldentification Index (computerized database) } \\
\text { * TSD Unit listing of retained record requirements } \\
\text { * TSD Unit listing of documents in its Administrative Record } \\
\text { * Coordination of information for the Public Information Repositories }\end{array}$ & B1,35,2 & & 163 & 4,352 & \\
\hline $\begin{array}{l}\text { RCRA Compliance (7330) } \\
\text { "1R1H75 - Annual Closure Cost Estimate and Waste Minimization } \\
\text { Reports } \\
\text { *Closure cost estimates are only needed for "final status" TSD } \\
\text { Units } \\
\text { * Waste Minimization Report includes all final status TSD Units }\end{array}$ & $B 1,35,2$ & & 292 & 4,644 & \\
\hline $\begin{array}{l}\text { RCRA Compliance }(7330) \\
\text { "1R1H76 - RCRA Permits Waste Inventory } \\
\text { " "Waste location, volume, and concentration by TSD Unit } \\
\text { " Incorporate changes to the WIDS and SWMU waste information }\end{array}$ & B1,35,2 & & 56 & 4,700 & \\
\hline
\end{tabular}




\begin{tabular}{|c|c|c|c|c|c|}
\hline Description (ADS) & $\begin{array}{l}\text { Program } \\
\text { Prioirty }\end{array}$ & $\begin{array}{c}\text { TPA } \\
\text { MS \# }\end{array}$ & $\begin{array}{l}\text { BCWS } \\
\text { Est. }\end{array}$ & $\begin{array}{l}\text { CUM } \\
\text { TOTAL }\end{array}$ & Comments \\
\hline $\begin{array}{l}\text { RCRA Compliance (7330) } \\
\text { "1R1H77 - Facility Wide Inspections } \\
\text { * *Epands the existing TSD Unit-regulator inspection process } \\
\text { * *Incorporates an inspection response process }\end{array}$ & $\mathrm{B1}, 35,2$ & & 161 & 4,861 & \\
\hline $\begin{array}{l}\text { Air and Water Permit Requirements (7330) } \\
\text { *1R1H41 - Air and Water Permitting Coordination } \\
\text { * "General Air/Water Permitting Coordination } \\
\text { * Provide State and Federal Regulatory Analysis Support } \\
\text { * RL Support for Wastewater Permits } \\
\text { * } \text { Stormwtr/UIC/Drinking Water Permit } \\
\text { * Air Emission Inventories } \\
\text { * *Air Operating Permit Application } \\
\text { * *F-01, NESHAP and Portable Permit Support }\end{array}$ & B1,35,2 & & 865 & 5,726 & . \\
\hline $\begin{array}{l}\text { Environmental Field Services }(7330) \\
\text { *1R1H31 - Environmental Field Services } \\
\text { * "Provide support for Facility Support Management } \\
\text { * Escort regulatory agency inspectors onsite } \\
\text { * "Inspection Support } \\
\text { * Environmental Compliance } \\
\text { * "Regulatory Compliance File Development }\end{array}$ & B1,35,2 & $M-32$ & 786 & 6,512 & \\
\hline $\begin{array}{l}\text { NEPA Coordination }(7330) \\
-1 R 1 H 21 \text { - NEPA Coordination } \\
\text { * NEPA Site Coordination }\end{array}$ & B1,35,2 & & 232 & 6,744 & \\
\hline $\begin{array}{l}\text { Tri-Party Agreement Integration (7330) } \\
\text { *1R1H61 - Tri-Party Agreement Integration } \\
\text { *Tri-Party Agreement Coordination/Business Integration Support } \\
\text { * Tri-Party Agreement Handbook Support } \\
\text { * Environmental Tracking System Support }\end{array}$ & B1,35,2 & & 814 & 7,558 & • \\
\hline $\begin{array}{l}\text { Tri-Party Agreement Integration (7330) } \\
\text { *1R1H61 - Tri-Party Agreement Integration } \\
\text { *Tri-Party Agreement Tactical Management }\end{array}$ & B1,35,2 & & 552 & 8,110 & \\
\hline
\end{tabular}




\begin{tabular}{|c|c|c|c|c|c|}
\hline 2L. Building Blocks & FY 1 & 996 & & & \\
\hline Description (ADS) & $\begin{array}{l}\text { Program } \\
\text { Prioirty }\end{array}$ & $\begin{array}{l}\text { TPA } \\
\text { MS \# }\end{array}$ & $\begin{array}{l}\text { BCWS } \\
\text { Est. }\end{array}$ & $\begin{array}{l}\text { CUM } \\
\text { TOTAL }\end{array}$ & Comments \\
\hline $\begin{array}{l}\text { FY96 TOTAL PIT PROPOSED TARGET LESS } \\
10 \% \text { PRODUCTIVITY CHALLENGE }\end{array}$ & & & 8,110 & & \\
\hline Budget Adjustment & $B 1,35,2$ & & 901 & 9,011 & \\
\hline FY 1996 TOTAL PIT PROPOSED TARGET & & & 9,011 & & \\
\hline $\begin{array}{l}\text { RCRA Compliance }(7330) \\
\text { "1R1H74 - Pipeline Mapping and Marking } \\
\text { * *Facility-wide mapping/marking of orphan pipelines }\end{array}$ & B1,35.2 & & 1.494 & 10,505 & \\
\hline $\begin{array}{l}\text { Pollution Prevention (7332) } \\
\text { *1R1H81 - Pollution Prevention (P2) } \\
\text { * *P2 Program Implementation } \\
\text {-*Hazardous Materials Reduction Act. }\end{array}$ & $B 2,35,2$ & & 714 & 11,219 & \\
\hline $\begin{array}{l}\text { RCRA Compliance }(7330) \\
\text { *2R1H79 - CENRTC } \\
\text { * Record Keeping Computer System }\end{array}$ & B1,35,2 & & 262 & 11,481 & \\
\hline $\begin{array}{l}\text { NEPA Coordination (7330) } \\
\text {-1R1H21 - NEPA Coordination } \\
\text { * Sitewide NEPA EIS }\end{array}$ & B1,35,2 & & 196 & 11,677 & \\
\hline $\begin{array}{l}\text { NEPA Coordination }(7330) \\
\text {-1R1H22 - NRDA Coordination } \\
\text { - NRDA Site Coordination }\end{array}$ & $B 1,35,2$ & & 219 & 11,896 & $\begin{array}{l}\text {-NOTE: Scope has been transferred to BHI } \\
\text { Should the HEMP be funding this activity? }\end{array}$ \\
\hline FY96 PLANNED BUDGET & & & 11,896 & & \\
\hline
\end{tabular}



WBS 1.5.2.1

Section 3

Fiscal Year Work Plan 
FY 1995 MYPP

HANFORD ENVIRONMENTAL MANAGEMENT PROGRAM

WBS 1.5.2.1

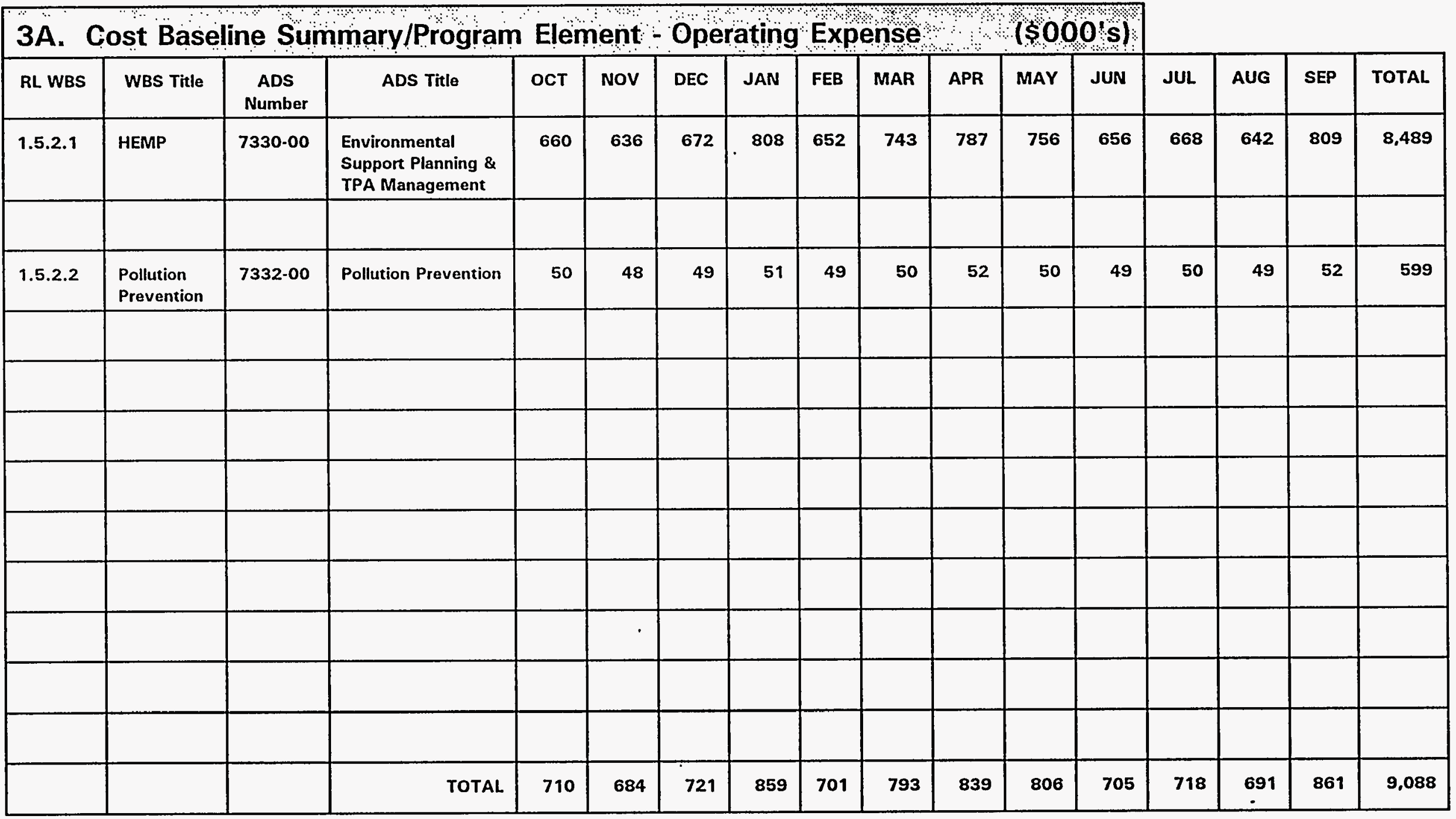


FY 1995 MYPP

HANFORD ENVIRONMENTAL MANAGEMENT PROGRAM

WHC-SP-1112

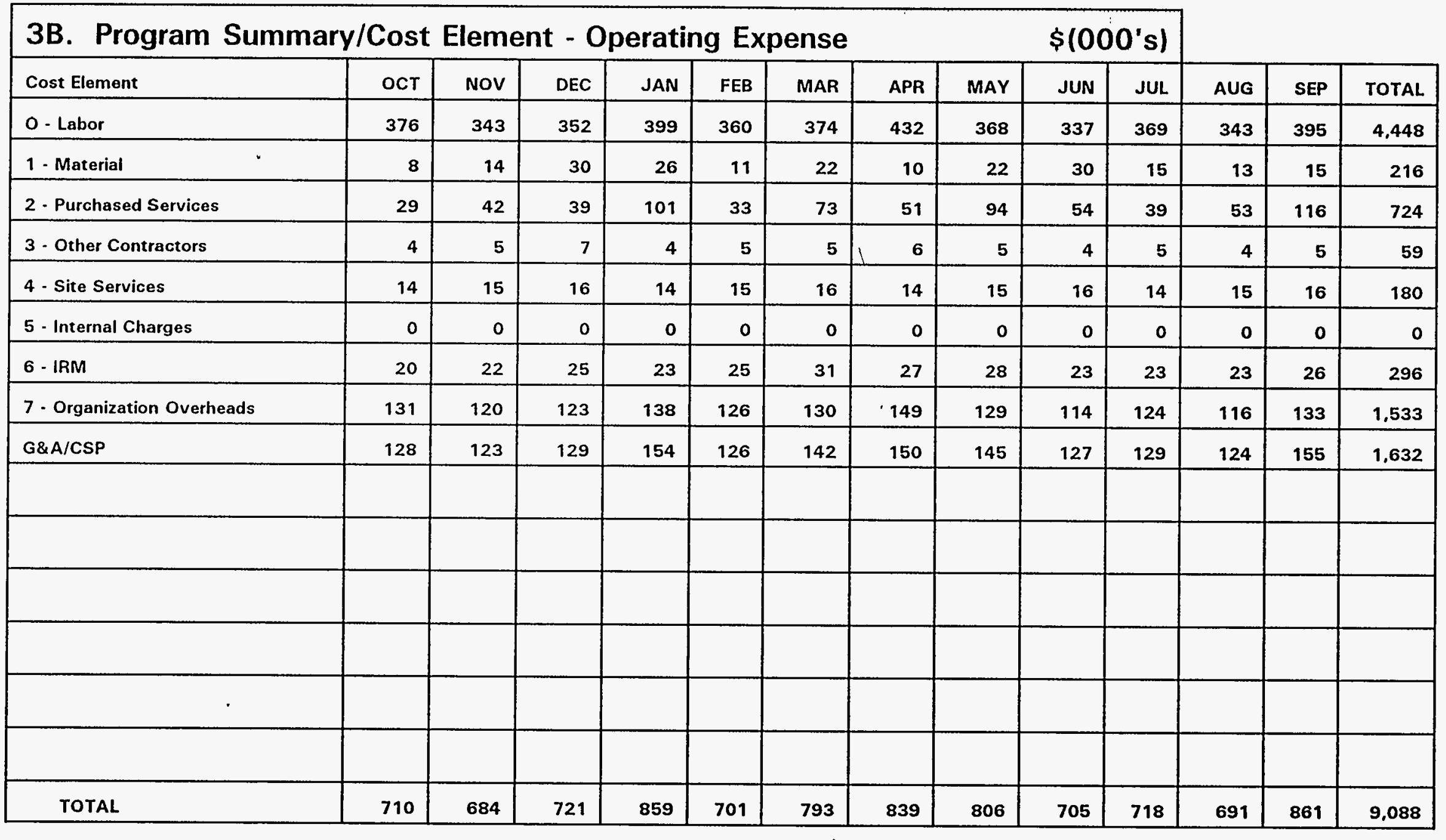


WBS 1.5.2.1

\section{C. Cost Baseline Summary - CENRTC}

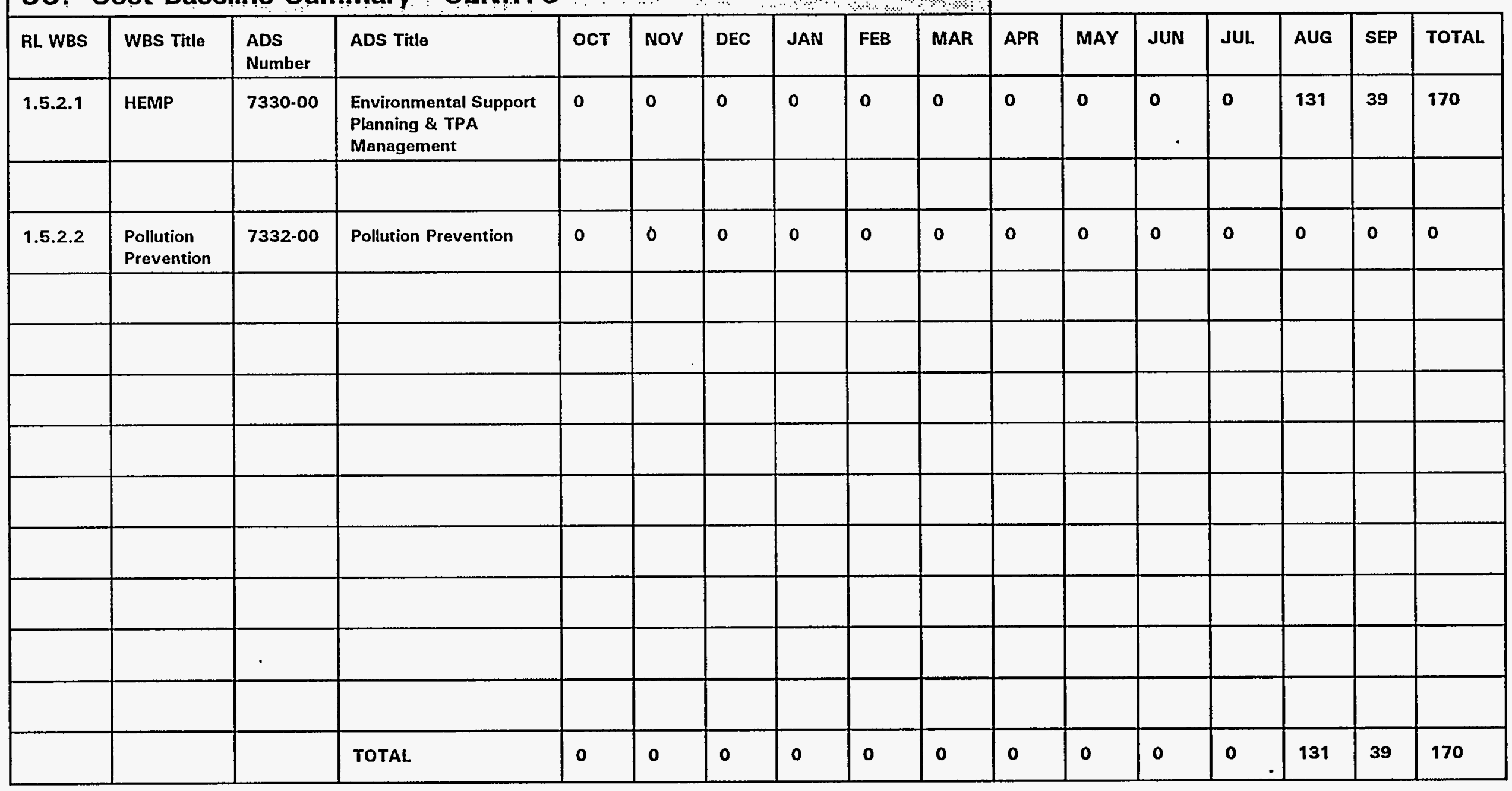


WBS 1.5.2.1

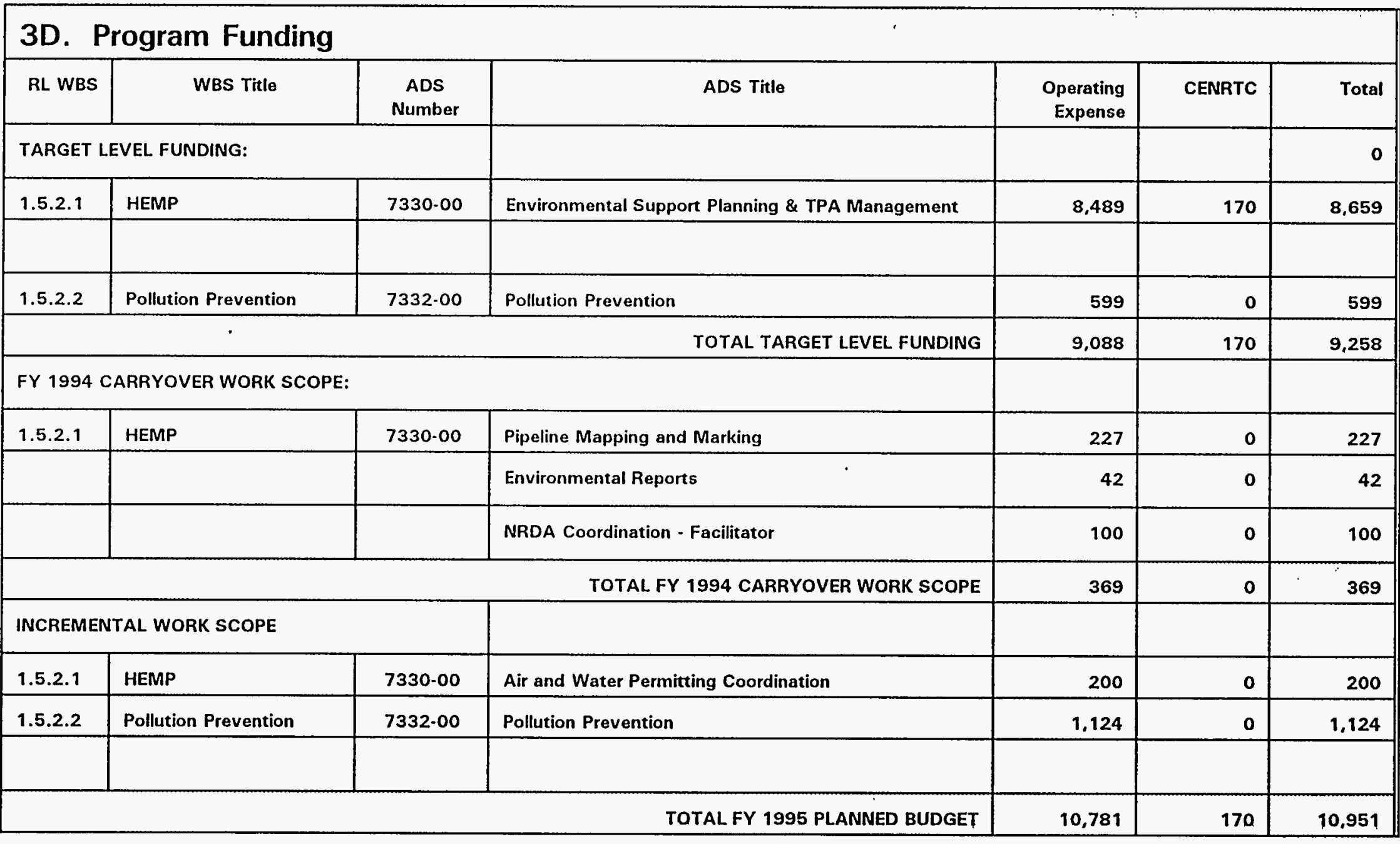


1.5.2 Environmental Support

U. S. Department of Energy

Richland Operations Office

\section{Environmental Support - Inventories Management WHC Program Manager}

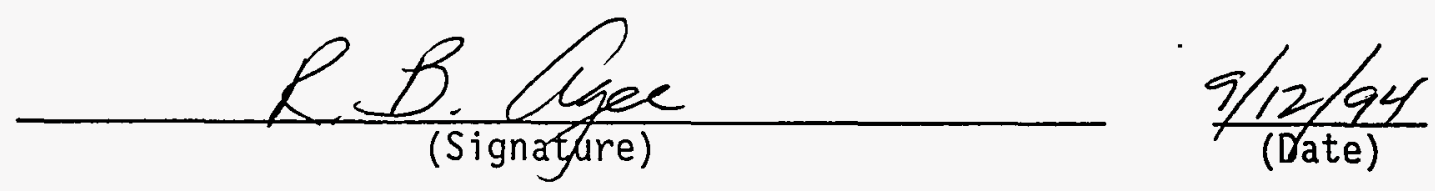

R. B Agee

(Printed Name) 
TABLE OF CONTENTS

1. Program Overview ................. 2-1

1A. Program Vision/Mission ............ 2-2

1B. Program Mission Strategy ........... 2-3 1B1. Technical Function and Requirements ... 2-4 - 2-5 1B2. Program Objectives .......... 2- 2-6

1C. P1anning Assumptions ............ 2-7

1D. Issue and Constraints ............. 2-8

2. Program Baseline .............. 2-. . . .

2A. WBS Informatiation ............... 2-10 2A1. WBS Structure ............ 2-11 2A2. WBS Dictionary ....... 2-12- 2-13

2B. Responsibility Assignment Matrix ......... 2-14

2C. Program Logic Diagram . . . . . . . . . 2-15

2D. Program Master Basel ine Schedule (PMBS) ....... 2-16

2E. Program Performance Baseline Schedule (PPBS) . . . 2-17

2F. Milestone List .............. 2-18

2G. Milestone Description Sheets .......... 2-19

2H. Cost Baseline Summary by Year . . . . . . . . . 2-20

2I. Basis of Estimate.............. 2-21

2J. Waste Type Data . . . . . . . 2-22-2-24

2K. Planned Staffing (Full-Time Equivalents) ..... 2-25

2L. Building Blocks........... 2-26-2-27

3. Fiscal Year Work Plan (FYWP) . . . . . . . . . 2-28

3A1. Cost Estimate Baseline by Month . . . . . . . . 2-29

$3 A 2$. Program Summary/Cost Element . . . . . . . . 2-30

3A3. Program Funding .............. 2-31 


\section{.}


$\frac{N}{5}$
$\frac{1}{5}$
$\frac{1}{3}$

$\frac{E}{4}$

$\infty$

ㄴ.

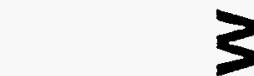

E

$4 m$

$-2$

$\sum$ S

15

$r{ }^{\infty}{ }^{\infty}$

0

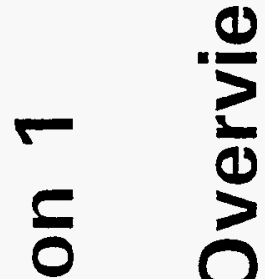

อ.

$\frac{D}{\infty}$

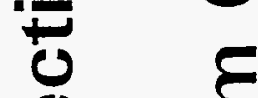

b)

(5)

0

a

$\sum$

0

$\sum \sum_{z}^{\frac{0}{2}}$

오

\%

(2)

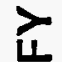




\section{ENVIRONMENTAL SUPPORT - INVENTORIES MANAGEMENT}

\section{A. Program Vision/Mission}

Provide disciplined, safe, environmentally sound and cost-effective processes that support Waste Management and Environmental Restoration inventory activities.

Maintain control of the physical inventory to ensure that any loss of inventory items are at or below the established thresholds. 


\section{B. Program Mission Strategy}

Provide administrative, warehousing and budget support to manage the Waste Management and Hanford Site inventories per DOE request while ensuring safe and efficient operations of facilities and equipment as they relate to the Hanford Mission.

Reduce/minimize the level of inventory (stocked on-hand) required to operate the Hanford Site, through planned reductions to current inventory of items. This includes items no longer required by the facilities/programs as a result of the change in the Hanford mission, obsolescence or modification.

Continue to aggressively review and remove from inventory, all items that meet the criteria for Systems Contracting. Items that are readily available by other means and are not critical to operations, will not be stocked in the site warehouses. 
$\frac{2}{5}$

$\frac{E}{2}$

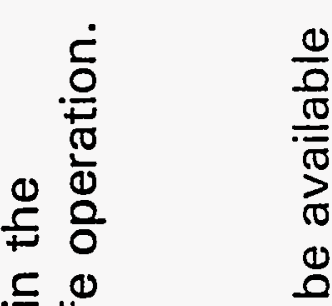

.$\frac{5}{\pi} \frac{0}{\pi}$

$\stackrel{+}{.}$

है ?

을

号

$\infty$

니

웡

บำ

ผ ㄷํㅇ

0

을 웅

$\sum m$

象离

ล

क

$\sum_{1}^{1}$

$\sum_{0}^{\sum}$ 0

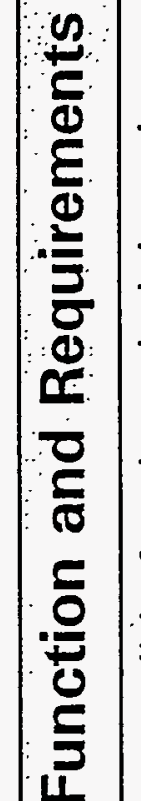

(1)

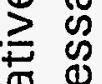

T.

我

.

हิํํㅇ

(1)

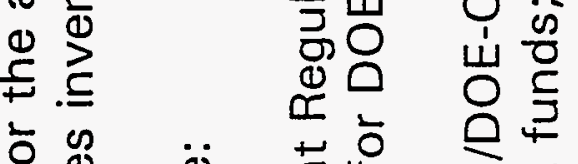

40 के

$\frac{0}{0}$

$\frac{0}{\omega}$ क

实

की पू

$\simeq \stackrel{E}{\leftarrow}$

\&

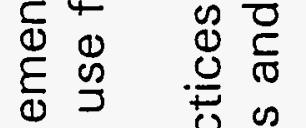

ठ $\subseteq$

高市

$\sum \frac{1}{E}$

邓을

4

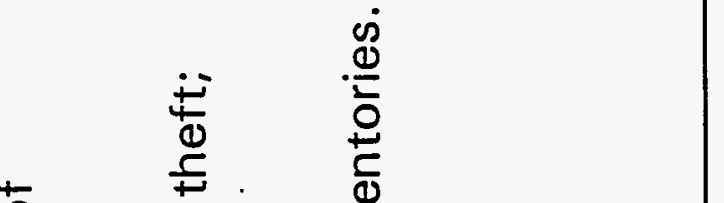

$\stackrel{+}{\mathfrak{L}}$

ํํㅇㅁㅇ

娄

రั

ล

뭉

E

苛衣

응 잉

O

亡

แ

$\omega \stackrel{0}{\circ}$

○ํำ

○ी

(

으

능

岁 गे

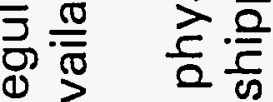

뜽

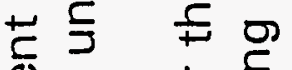

๕⿺辶

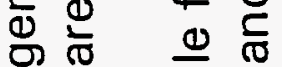

흥 을

तั

त क के.

๖

으린

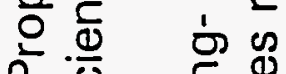

ด. $\frac{0}{0 .}$

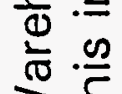

$\infty \sum^{\infty} \cdot \frac{E}{E} \stackrel{\Phi}{\Phi}$

$\sum E$ 
WBS 1.5.2.3

\section{B1. Technical Function and Requirements}

Requirements documents include:

DOE Property Management Regulation/41CFR - Improper use of materials; theft; inefficient use of spares; unavailability of materials.

DOE 4330.4A - Segregation of inventory items; inventory shelf-life control program; traceability of essential materials/chemicals

Procure new and replacement process spare parts to support continuity of Waste Management Operations. This scope includes procuring spares for capital equipment and construction projects and spares to support Redox, Tank Farms, Powerhouse, Landlord, 222-S Labs, Grout, Liquid Effluent Retention, Water Export Electrical Maintenance, Asbestos Abatement, T-Plant and Core Sampling Activities.

Maintaining appropriate inventory for process spares may determine whether Tri-Party Agreement Milestones are completed as scheduled (examples are Powerhouse and Export Water facility projects). Spares inventory items are used for repairs and installation of systems that decrease/eliminate and monitor effluent discharges into the air and water.

Spares are used for the maintenance and repair of steam emergency equipment (raw water pumps for fire systems), sanitary water pumps and steam systems. Export water spares 


\section{ENVIRONMENTAL SUPPORT - INVENTORIES MANAGEMENT

\section{B2. Program Objectives}

Maintain the minimum required inventory levels to ensure the continued, safe operation of the facilities supported.

Reduce inventory categories related to the defense mission and those inventory items no longer required as a result of obsolescence.

Comply with applicable DOE Orders and regulations set forth in WHC manuals.

Provide and administer the Hanford Inventory Program (HIP) database.

Provide training for users of HIP system and administrative requirements for the cognizant engineer to implement appropriate inventory procedures.

Report monthly to DOE through Site Management and Progress Tracking Systems.

Implement systems contracts where possible.

Provide overall liaison activities for all Westinghouse financially controlled inventories on the behalf of DOE-RL. 


\section{C. Planning Assumptions}

Minimal growth in the inventory change funding and $0 \%$ in the administrative management of this inventory ADS.

Escalation is adequate relative to the funding level required to maintain compliance with DOE Orders.

The continued reduction of inventory items rendered obsolete by the change in mission will off-set the "new item" requirements.

Reduced inventory change will mean an increase in direct purchase orders being placed by programs which will affect material availability.

Direct orders that are brought on-site by programs will be stored outside of the financial controlled inventory warehouses. 


\section{D. Issues and Constraints}

Inventories are a requirement of DOE Order 4330.4A as called out in Paragraph 8. Per this order it is the Department's policy that the maintenance management program for all DOE facilities be consistent with this order and that the property is maintained in a manner which promotes, operational safety, worker health, environmental protection and compliance, property preservation, and cost effectiveness while meeting the programmatic mission.

The DOE order maintenance management objectives are to: Ensure the reliability, safety, and operability of structures, systems, and components. In addition to the above requirement, the order also specifies as an objective to: Ensure that sufficient resources are designated and budgeted in a timely manner to accomplish the maintenance program objectives.

DOE Order 4330.4A, Paragraph 3.5.3, identifies effective material control as: practical and is achieved when the right materials in the correct quantities are delivered to the job location at the proper time.

If inventories are not funded, Westinghouse potentially will be in violation of DOE Order 4330.4A...Maintenance Management Program. In addition, there will be potential violations with regard to the WAC, EPA, CERCLA, RCRA, TRI-PARTY AGREEMENT. 
$\frac{1}{5}$
$\frac{1}{5}$
$\frac{5}{5}$

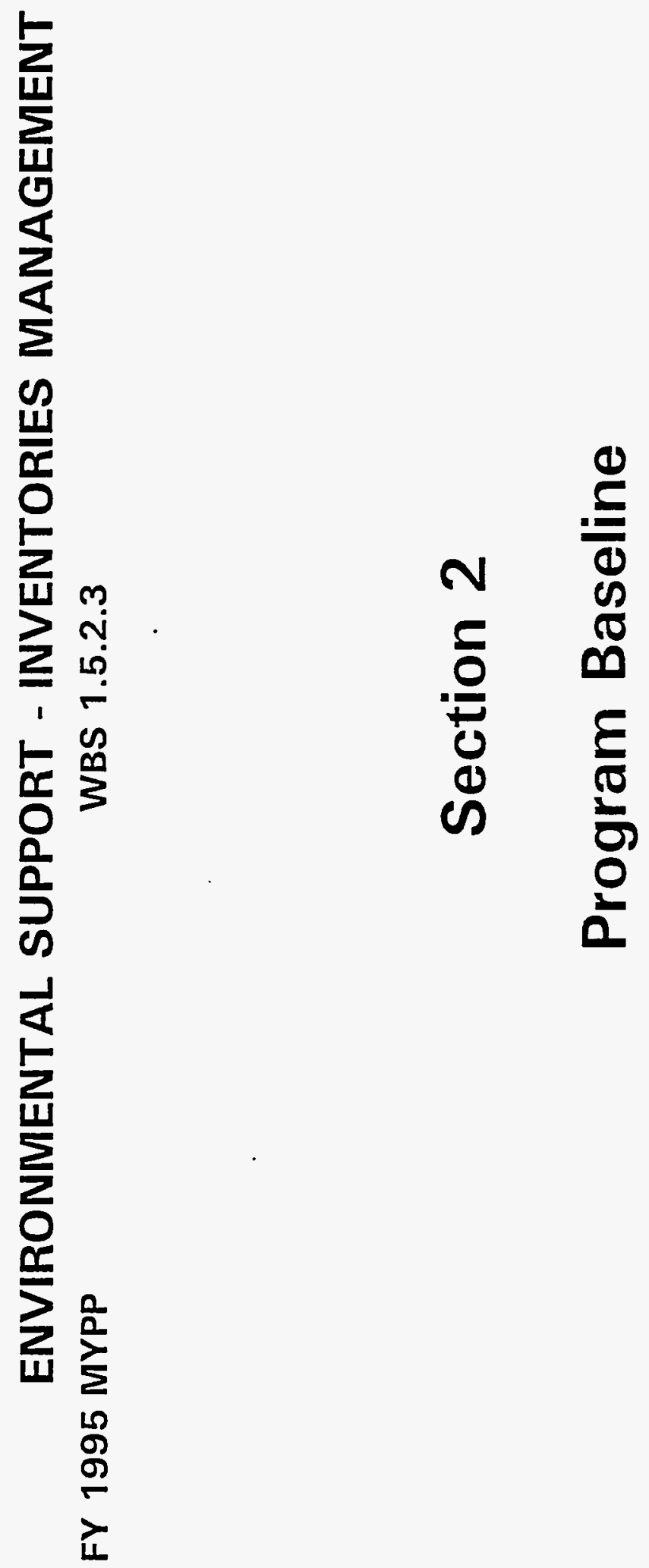




\section{A. WBS Information}

This section describes the Environmental Support - Inventories Management requirements and responsibilities for the program tasks into a logical breakdown of work such that the work can be effectively defined, planned, scheduled, budgeted, assigned, performed, measured, and controlled. 


\section{ENVIRONMENTAL SUPPORT - INVENTORIES MANAGEMENT}

\section{WBS 1.5.2.3}

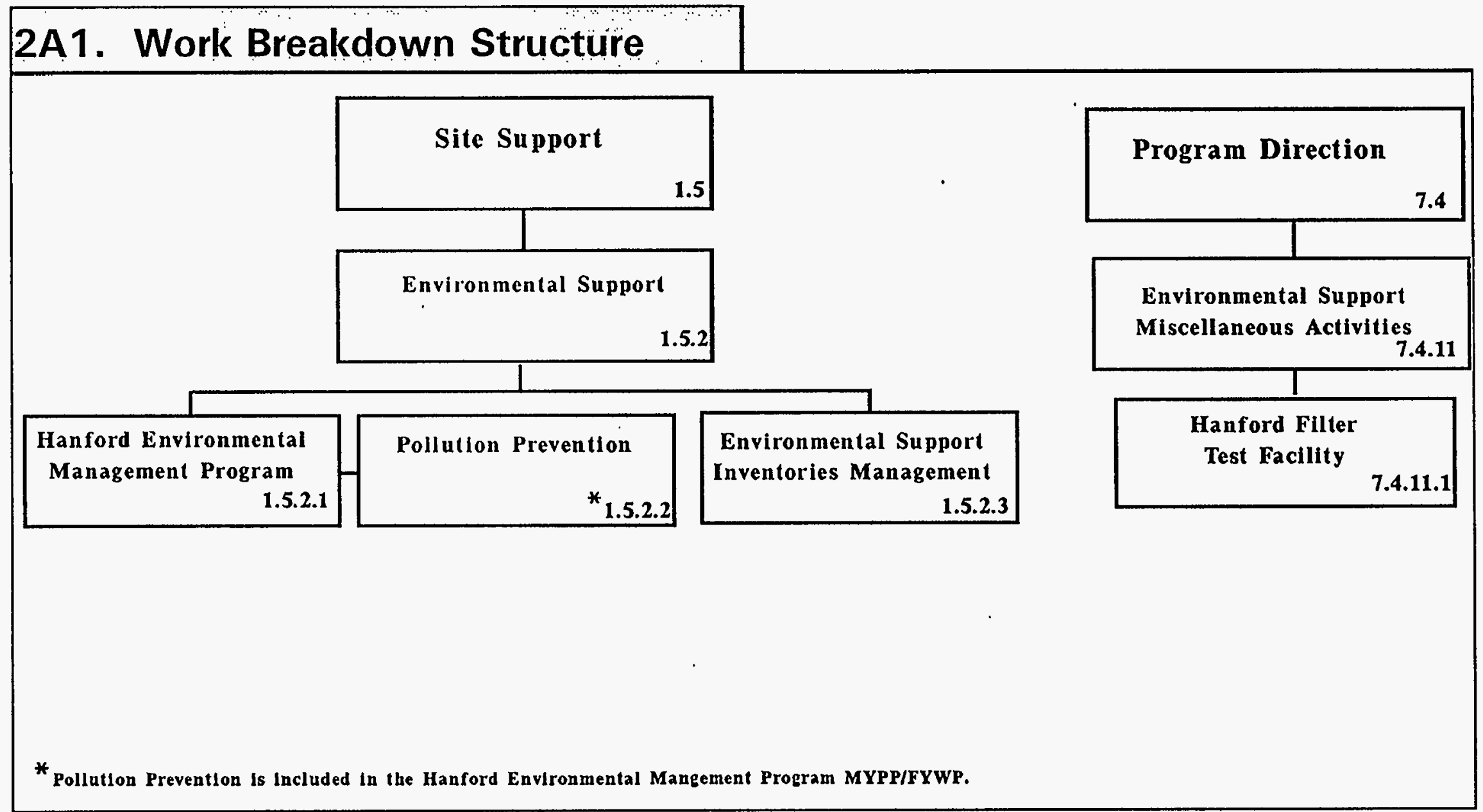




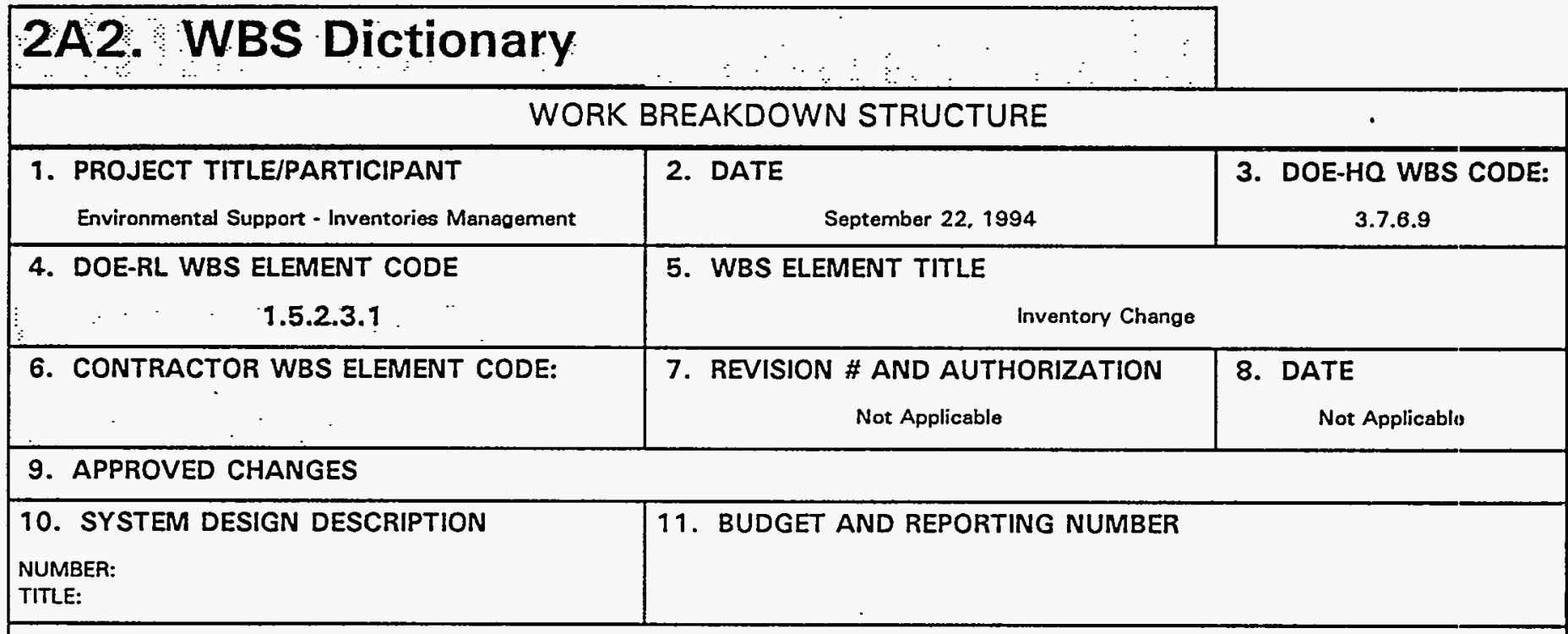

12. ELEMENT TASK DESCRIPTION - This activity deals specifically with changes to the inventory accounts as they relate to increases, decreases, transfers, and establishment of new inventory items.

\section{A. COST CONTENT}

Inventory change

Inventory change is the total growth allowed for each of the accounts above and beyond the fiscal year-end inventory balance. This growth can be experienced in the form of newiy established materials, components, or items required as a result of new facilities, facility modifications, obsolete inventory or an increase in the stocking level of a pre-existing inventory item.

\section{B. TECHNICAL CONTENTMORK STATEMENT}

1. Includes WBS Elements

\section{B\&R Code EW3180010}

1.5.2.3.1.1 - Coal Inventory Change

1.5.2.3.1.2 - Essential Materials/Chemicals

1.5.2.3.1.3 - General Supplies/Computer Hardware and Software

1.5.2.3.1.4 - Fuels/Miscellaneous

1.5.2.3.1.5 - Miscellaneous Radio Equipment

1.5.2.3.1.7 - Telecommunications Spare Parts

B\&R Code EW3180040

\subsection{6 - Process Spare Parts}

2. Identification and implementation of systems contracts to reduce the need to stock specific inventory items.

Reduction of inventory meets a main objective of the program mission.

3. Thoroughly challenge all requests for new inventory items for each of the inventory accounts listed above.

4. Identify inventory for excessing in each of the above listed inventory accounts. This activity is to include a monthly report detailing the number of items in each inventory account (fiscal year baseline), number of candidate items up for review and a detailed breakout of the review results in monetary as well as line item value. 


\section{A2. WBS Dictionary}

WORK BREAKDOWN STRUCTURE

\begin{tabular}{|c|c|c|}
\hline $\begin{array}{l}\text { 1. PROJECT TITLE/PARTICIPANT } \\
\text { Environmental Support - Inventories Management }\end{array}$ & September 22, 1994 & $\begin{array}{l}\text { 3. DOE-HO WBS CODE: } \\
3.7 .6 .9\end{array}$ \\
\hline $\begin{array}{l}\text { 4. DOE-RL WBS ELEMENT CODE } \\
\text { \% }\end{array}$ & $\begin{array}{l}\text { 5. WBS ELEMENT TITLE } \\
\text { Inventory Change (Adm }\end{array}$ & ative) \\
\hline 6. CONTRACTOR WBS ELEMENT CODE: & $\begin{array}{l}\text { 7. REVISION \# AND AUTHORIZATION } \\
\text { Not Applicable }\end{array}$ & $\begin{array}{l}\text { 8. DATE } \\
\text { Not Applicablo }\end{array}$ \\
\hline
\end{tabular}

9. APPROVED CHANGES

10. SYSTEM DESIGN DESCRIPTION

11. BUDGET AND REPORTING NUMBER

12. ELEMENT TASK DESCRIPTION - This activity addresses the management, administration, warehousing, delivery, planning and reporting of inventories. These tasks are required to assure the safe-keeping, availability and traceability of government property for the Department of Energy.

\section{A. COST CONTENT}

Inventory Administrative Support

\section{B. TECHNICAL CONTENT/WORK STATEMENT}

1. Includes WBS Elements

B\&R Code EW3110010

1.5.2.3.2.1 - Process Spares Administration

1.5.2.3.2.2 - Program Support

1.5.2.3.2.3 - Warehousing Administration

1.5.2.3.2.4 - Warehousing (Occupancy)

1.5.2.3.1.8 - Allowance for Loss, Damage or Obsolescence (Reserve)

2. The Cost account Manager for each of the above listed accounts is to provide a monthly report detailing issues, concerns, accomplishments and variance explanations as required in WHC-CM-2-5, Section 3.1, Data Analysis and Variance Reporting. The input provided will be for reporting through the Site Management System (SMS) and Progress Tracking System (PTS) reporting

3. Preparation of Activity Data Sheets and Multi-Year Program Planning documents.

4. Issuance of the Spare Parts Annual Review report to facility managers shall be performed by Material Control. Each facility inventory shall be reviewed at least annually. This effort shall be scheduled to ensure that the workload is spread-out over the twelve month fiscal year. A minimum of one facility each month shall be issued for review. This will ensure continual reductions are requested. This activity is to include a monthly report detailing the number of items in each inventory account (fiscal year baseline), number of candidate items up for review and a detailed breakout of the review results in monetary value as well as line item count.

5. Perform annual justification of convenience storage materials. Items not adequately justified will be removed. Site Warehousing conducts this activity.

6. Conduct classes to train personnel in the maintenance and control of spares inventory - includes ordering supplies, excessing, establishing new items, and accessing the Hanford Inventory Program (HIP) Database. Material Control schedules and conducts this training as requested by the customers at facility level.

7. Ensure safe storage of inventories. 
ENVIRONMENTAL SUPPORT - INVENTORIES MANAGEIMENT

\begin{tabular}{|c|c|c|c|c|c|}
\hline PROGRAM ELEMENT & ACTIVITY & COST ACCOUNT & TITLE & RESPONSIBLE MANAGER & RESPONSIBLE ORGANIZATION \\
\hline \multirow[t]{15}{*}{1.5 .2 .3} & & & $\begin{array}{l}\text { Environmental Support Inventories } \\
\text { Management }\end{array}$ & RB Agee & Financial Planning and Integration \\
\hline & 1 1.5.2.3.1 & & Inventory Change & N/A & N/A \\
\hline & & 1.5 .2 .3 .1 .1 & Coal Inventory & JL Day & Stm \& Wtr Util \\
\hline & & 1.5 .2 .3 .1 .2 & Essential Materials \& Chemicals & RE Boykin & Material Control \\
\hline & & 1.5 .2 .3 .1 .3 & General Supplies \& Computers & RE Boykin & Gen Sup Inv Ctl \\
\hline & & 1.5 .2 .3 .1 .4 & Fuels/Miscellaneous & JF Hoods & Fleet op \& Maint \\
\hline & & 1.5.2.3.1.5 & Miscellaneous Radio Equipment & LA Ahrens & IRM Admin \\
\hline & & 1.5 .2 .3 .1 .6 & Process Spare Parts & RE Boykin & Material Control \\
\hline & & 1.5.2.3.1.7 & Telecommunications Spare Parts & RE Boykin & Material Control \\
\hline & & 1.5 .2 .3 .1 .8 & Allowance for Loss (Reserve) & RB Agee & Financial Planning and Integration \\
\hline & 1.5 .2 .3 .2 & & Inventory Administration & N/A & N/A \\
\hline & & 1.5 .2 .3 .2 .1 & Process Spares Administration & RE Boykin & Material Control \\
\hline & & 1.5 .2 .3 .2 .2 & Program Support & RB Agee & Financial Planning and Integration \\
\hline & & 1.5 .2 .3 .2 .3 & Warehousing Adninistration & HC Humphery & Material Management \\
\hline & & 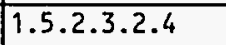 & Warehous ing (Occupancy) & RB Agee & Financial Planning and Integration \\
\hline & & & & & \\
\hline & & & & & \\
\hline & & & & & \\
\hline & & & & & \\
\hline & & & & & \\
\hline
\end{tabular}




\section{C. Program Logic Diagram}

N/A 
ENVIRONMENTAL SUPPORT - INVENTORIES MANAGEMENT WHC-SP-1112 FY 1995 MYPP WBS 1.5.2.3

2D. Program Master Baseline Schedule (PMBS)

N/A 
ำ
5
0
0
5
3

$\frac{N}{2}$

$\frac{1}{11}$

足

更

$\sum_{m}^{\infty}$

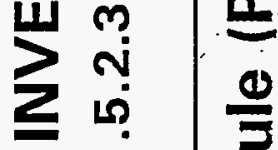

$1 \stackrel{\sim}{\circ}$

$0 \overbrace{}^{\infty} \frac{1}{0}$

$\frac{2}{\infty}$

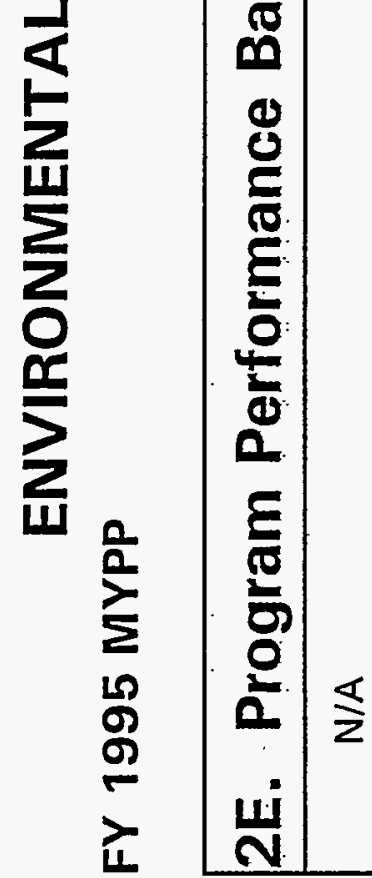


FY 1995 MYPP

WBS 1.5.2.3

\section{F. Milestone List}

N/A 


\section{G. Milestone Description Sheets} N/A 
ENVIRONMENTAL SUPPORT - INVENTORIES MANAGEMENT

FY 1995 MYPP

WBS 1.5.2.3

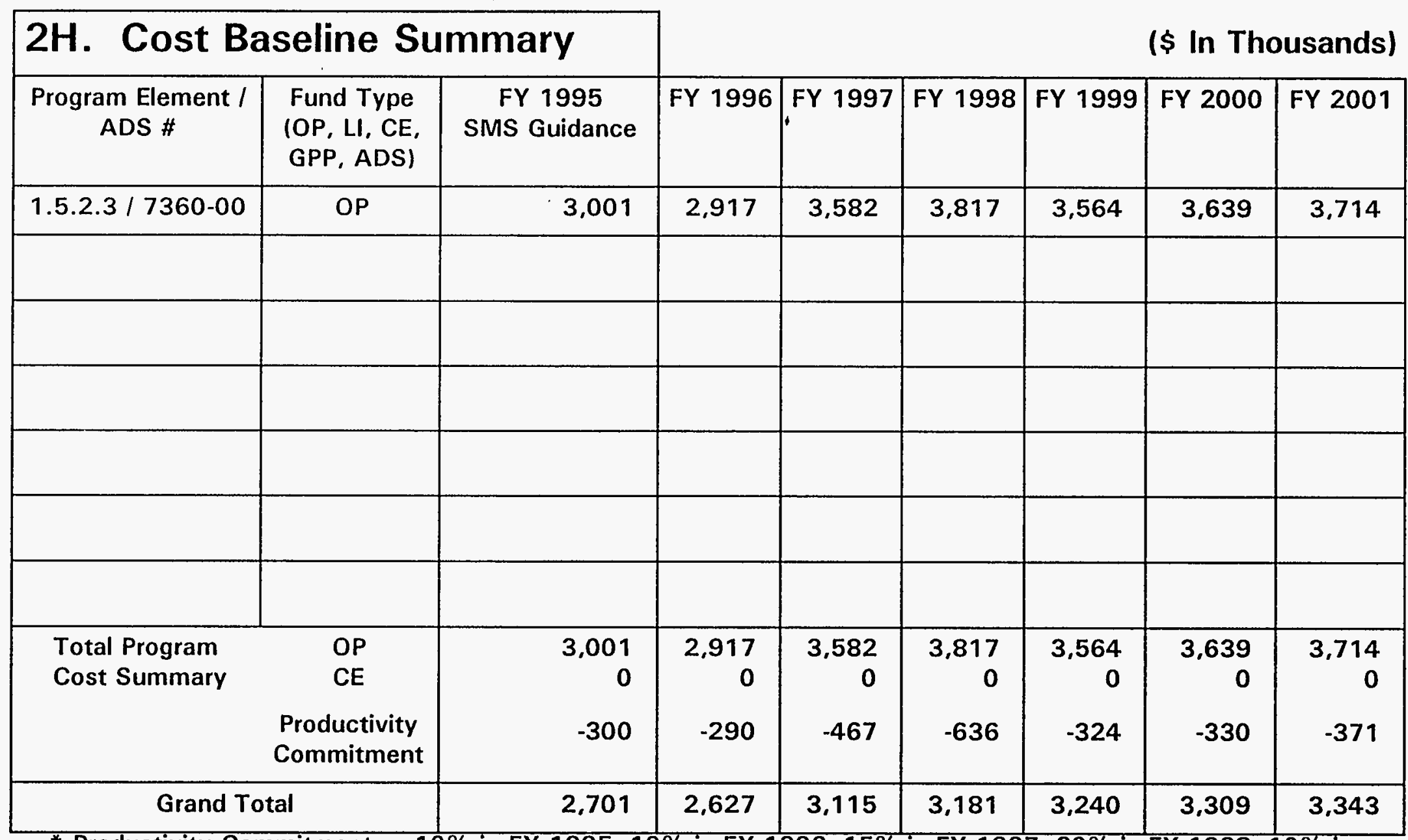

* Productivity Commitment $=10 \%$ in FY 1995, $10 \%$ in FY 1996, 15\% in FY 1997, 20\% in FY 1998, $10 \%$ in FY 1999, 10\% in FY 2000, 10\% in FY 2001. 
WBS 1.5.2.3

\section{I. BASIS OF ESTIMATE}

1 R51 - Inventory Change

Estimates for inventory change are based on input from the field/cognizant engineer, cost account managers the cognizant project control analyst and prior years cost account performance. Additionally, inventory growth is predicated on the assumption that the Hanford Site will continue to receive funding for the purpose of supporting the environmental clean-up mission. As a result, existing facilities, modified facilities/processes, new facilities, technological advancements and changing quality requirements influence, affect and create the need for inventory change.

1 R52 - Inventory Administration

Estimates based on established site rates for building occupancy (includes janitorial and utility services to $2101 \mathrm{M}$ and $275 \mathrm{EA}$ Buildings where process spares and chemicals are stored) and on actual cost account historical data of personnel required (managers, analysts, material control specialists, storekeepers, truck drivers and clerks) to administer, report, train, ensure the safe storage and segregation of materials safe keep and transport inventory items. 


\section{ENVIRONMENTAL SUPPORT - INVENTORIES MANAGEMENT WHC-SP-1112}

FY 1995 MYPP WBS 1.5.2.3

\section{J. Waste Type Data}

Additional Waste Storage, Treatment, and Disposal Capability Needs

- N/A

Waste Storage/Treatment, Disposal Issues

- N/A 


\begin{tabular}{|c|c|c|c|c|c|c|c|c|c|}
\hline \multicolumn{3}{|c|}{ 2J. Waste Type Dáta } & \\
\hline \multicolumn{10}{|c|}{ Projected Waste Volume Generation (cubic feet, gallons) } \\
\hline \multirow[t]{2}{*}{ Waste Type } & \multicolumn{2}{|c|}{ FY 1994} & \multicolumn{2}{|c|}{ FY 1995} & \multicolumn{2}{|c|}{ FY 1996} & \multicolumn{2}{|c|}{ FY97-2018 } & \multirow{2}{*}{$\begin{array}{l}\text { Planned TSD } \\
\text { Facility / Program }\end{array}$} \\
\hline & Liq. & Solid & Liq. & Solid & Liq. & Solid & Liq. & Solid & \\
\hline HLW (Tank Waste) & 0 & 0 & 0 & 0 & 0 & 0 & 0 & 0 & N/A \\
\hline TRU Waste & 0 & 0 & 0 & 0 & 0 & 0 & 0 & 0 & N/A \\
\hline LLW & 0 & 0 & 0 & 0 & 0 & 0 & 0 & 0 & N/A \\
\hline Mixed LLW & 0 & 0 & 0 & 0 & 0 & 0 & 0 & 0 & N/A \\
\hline Hazardous Waste & 0 & 0 & 0 & 0 & 0 & 0 & 0 & 0 & $N / A$ \\
\hline Sanitary Waste & 0 & 0 & 0 & 0 & 0 & 0 & 0 & 0 & N/A \\
\hline
\end{tabular}




\section{ENVIRONMENTAL SUPPORT - INVENTORIES MANAGEMENT}

FY 1995 MYPP

WBS 1.5.2.3

\begin{tabular}{|c|c|c|c|c|c|c|c|c|c|}
\hline \multicolumn{3}{|c|}{ 2J. Waste Type Data } & Oth & Facilit & (cul & feet, & jallon & & \\
\hline \multirow[t]{2}{*}{ Waste Type } & \multicolumn{2}{|c|}{ FY 1994} & \multicolumn{2}{|c|}{ FY 1995} & \multicolumn{2}{|c|}{ FY 1996} & \multicolumn{2}{|c|}{ FY97-2018 } & \multirow{2}{*}{$\begin{array}{l}\text { Planned TSD } \\
\text { Facility / Program }\end{array}$} \\
\hline & Liq. & Solid & Liq. & Solid & Liq. & Solid & Liq. & Solid & \\
\hline HLW (Tank Waste) & 0 & 0 & 0 & 0 & 0 & 0 & 0 & 0 & N/A \\
\hline TRU Waste & 0 & 0 & 0 & 0 & 0 & 0 & 0 & 0 & N/A \\
\hline LLW & 0 & 0 & 0 & 0 & 0 & 0 & 0 & 0 & N/A \\
\hline Mixed LLW & 0 & 0 & 0 & 0 & 0 & 0 & 0 & 0 & N/A \\
\hline Hazardous Waste & 0 & 0 & 0 & 0 & 0 & 0 & 0 & 0 & N/A \\
\hline Sanitary Waste & 0 & 0 & 0 & 0 . & 0 & 0 & 0 & 0 & N/A \\
\hline
\end{tabular}




\section{ENVIRONMENTAL SUPPORT - INVENTORIES MANAGEIMENT}

FY 1995 MYPP

WBS 1.5.2.3

\begin{tabular}{|c|c|c|c|c|c|c|c|}
\hline 2K. Planined Staffing & 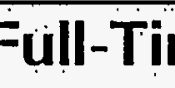 & Equil & ents) & & Job & nily only & fter 1997 \\
\hline JOB FAMILY & & & & & & & \\
\hline Job category & 1995 & 1996 & 1997 & 1998 & 1999 & 2000 & 2001 \\
\hline ADMIN/OTHER PROFESSIONALS TOT & 5.3 & 5.3 & 5.3 & 5.3 & 5.3 & 5.3 & 5.3 \\
\hline Accountant/auditor & 0.5 & 0.5 & 0.5 & 0.5 & 0.5 & 0.5 & 0.5 \\
\hline $\begin{array}{l}\text { Other - Material Control } \\
\text { spec }\end{array}$ & 4.8 & 4.8 & 4.8 & 4.8 & 4.8 & 4.8 & 4.8 \\
\hline GEN ADM/SECRETARY/CLERK TOTAL & 2.5 & 2.6 & 2.6 & 2.6 & 2.6 & 2.6 & 2.6 \\
\hline Admin Assistants & 0.4 & 0.5 & 0.5 & 0.5 & 0.5 & 0.5 & 0.5 \\
\hline Office Clerks (Gen) & 2.1 & 2.1 & 2.1 & 2.1 & 2.1 & 2.1 & 2.1 \\
\hline LABOR \& GEN WORKERS TOTAL & 4.4 & 4.4 & $\therefore 4: 4$ & 4.4 & 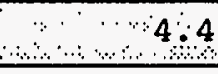 & 4.4 & 344 \\
\hline Other - Storekeepers & 4.4 & 4.4 & 4.4 & 4.4 & 4.4 & 4.4 & 4.4 \\
\hline$\cdot$ & & & \%०\% & \%। & ओ & $\because$ & ४। \\
\hline & & TOTALS B & ATEGORTE & \% & e & $\therefore$ & $\because \cdots$ \\
\hline EXEMPT & 5.3 & 5.3 & 5.3 & 5.3 & 5.3 & 5.3 & 5.3 \\
\hline NON-EXEMPT NON BARGAINING & 2.5 & 2.6 & 2.6 & 2.6 & 2.6 & 2.6 & 2.6 \\
\hline BARGAINING & 4.4 & 4.4 & 4.4 & 4.4 & 4.4 & 4.4 & 4.4 \\
\hline GRAND TOTALS & 12.2 & 12.3 & 12.3 & 12.3 & 12.3 & 12.3 & 12.3 \\
\hline
\end{tabular}


ENVIRONIMENTAL SUPPORT - INVENTORIES MANAGEMENT

WBS 1.5.2.3

\begin{tabular}{|c|c|c|c|c|c|c|c|}
\hline 2L. BL & ding Blocks & & & FY 1995 & & & $(\$$ in 000$)$ \\
\hline ADS & Description & $\begin{array}{l}\text { RL } \\
\text { Pri }\end{array}$ & TPA MS \# & Fund Type & FY 1995 & $\begin{array}{l}\text { CUM FY } \\
1995\end{array}$ & $\begin{array}{l}\text { Justification of Scope I } \\
\text { Impact if not funded }\end{array}$ \\
\hline $7360-00$ & Process Spare Parts & A2 & N/A & Expense & 589 & 589 & $\begin{array}{l}\text { Provide parts for Waste Operations/Facilities to assure } \\
\text { operation safety and efficiency. Additional spares are used for } \\
\text { planned new projects, new equipment and plant upgrades. }\end{array}$ \\
\hline $7360-00$ & Coal & A2 & N/A & Expense & 27 & 616 & Need to power 300 Area Steam Plant. \\
\hline $7360-00$ & Fuel/Misc. & A2 & N/A & Expense & 0 & 616 & Support infrastructure to roads, rails, and fuel for vehicles. \\
\hline $7360-00$ & Essential Materials/Chemicals & B2 & N/A & Expense & 25 & 641 & Includes drums to store waste. \\
\hline $7360-00$ & Telecommunications Spares & C1 & N/A & Expense & 50 & 691 & Parts for faxes, computers, radios, and telephone equipment. \\
\hline $7360-00$ & Radio Equipment & C1 & $N / A$ & Expense & $\mathbf{5}$ & 696 & Radio equipment including cellular phones. \\
\hline $7360-00$ & General Supplies/Computers & $\mathrm{C2}$ & N/A & Expense & 365 & 1,061 & $\begin{array}{l}\text { Includes office furniture, computer hardware/software, } \\
\text { essential items for fire protection and medical supplies. }\end{array}$ \\
\hline $7360-00$ & Process Spares Administration & D2 & N/A & Expense & 394 & 1,455 & $\begin{array}{l}\text { Administration, planning, reporting and training for process } \\
\text { spares, telecommunications spares, and essential materials. } \\
\text { Includes reorders, shelf criteria, assist in annual physical } \\
\text { inventories, and perform spares annual review. }\end{array}$ \\
\hline $7360-00$ & Program Support & D2 & N/A & Expense & 48 & 1.503 & $\begin{array}{l}\text { Monthly reporting of PTS and SMS. Preparation of EAC and } \\
\text { ADS Five-Year Plan, and MYPP. }\end{array}$ \\
\hline $7360-00$ & Warehouse Administration & D2 & N/A & Expense & 576 & 2,079 & Warehousing, receiving, and transportation of inventories. \\
\hline 7360.00 & Reserve Allowance for Loss & D2 & N/A & Expense & 99 & 2,178 & $\begin{array}{l}\text { Set aside reserve for inventory losses from damage, disposal, } \\
\text { excess, shrinkage, or deterioration. }\end{array}$ \\
\hline $7360-00$ & Warehouse Occupancy & D2 & N/A & Expense & 823 & 3,001 & Occupancy of $2101 \mathrm{M} \& 275 E A$. \\
\hline
\end{tabular}

Sub-Total Operating 3,001

Productivity Commitment $\quad-300$

TOTAL OPERATING $\quad 2,701$ 


\section{ENVIRONMENTAL SUPPORT - INVENTORIES MANAGEMENT}

FY 1995 MYPP

WBS 1.5.2.3

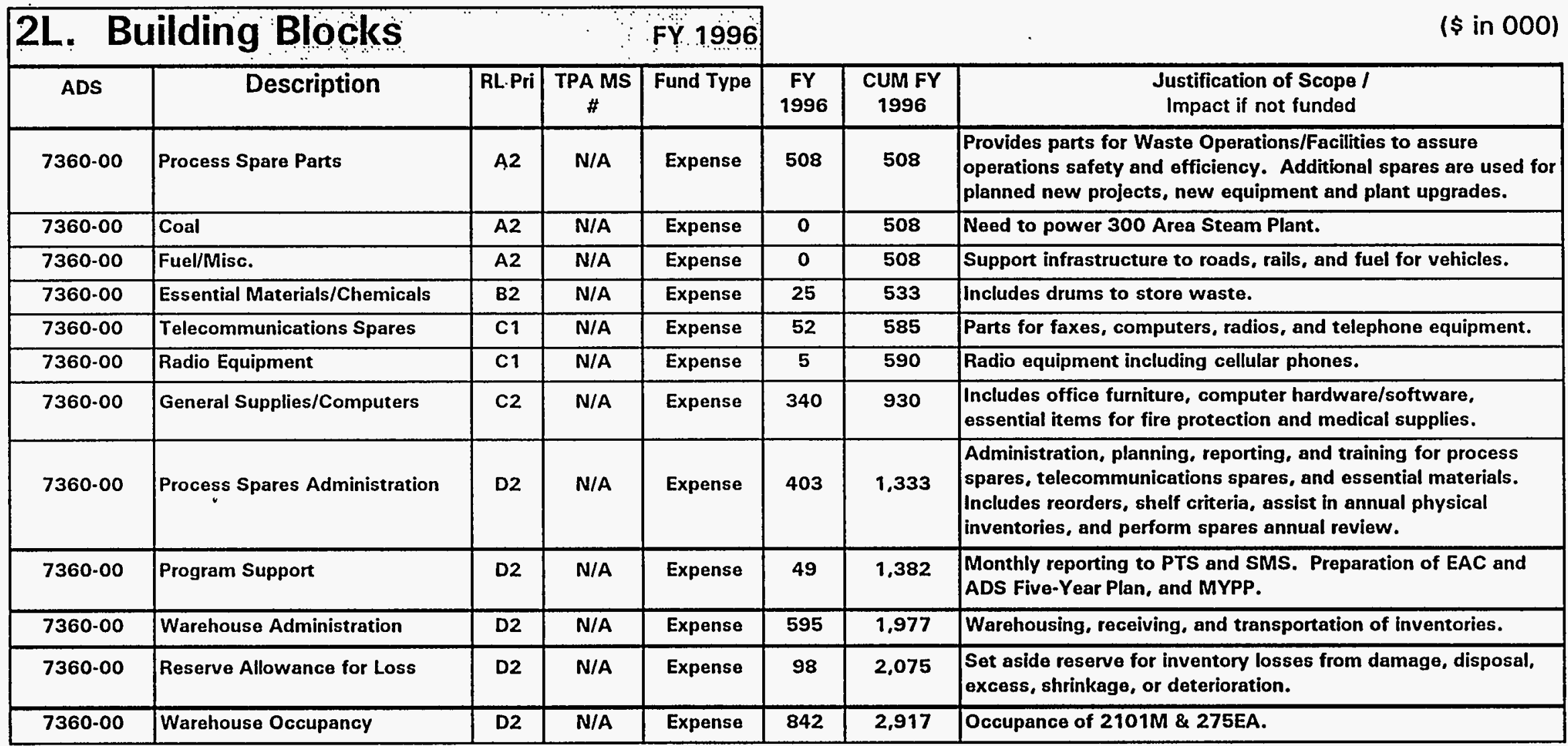

Sub- Total Operating 2,917

Productivity Commitment

Grand Total 2,627 
ㅁ
$\frac{1}{0}$
$\frac{1}{0}$
$\frac{1}{3}$

$E$
$\frac{2}{4}$
2
$z$

ऽ

U1

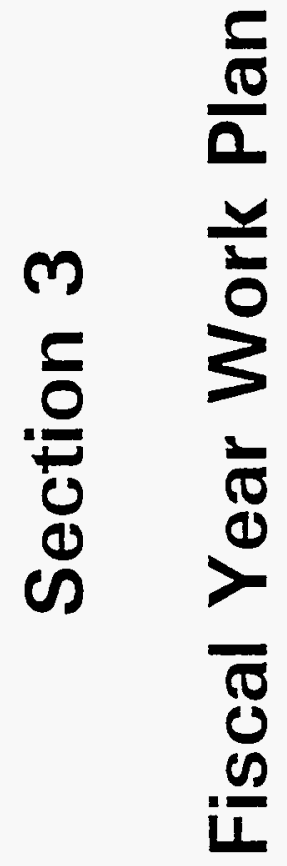

1

E

$\sum$

웅

r

$\sum$

늠

2

م

\%

5

i. 
ENVIRONMENTAL SUPPORT - INVENTORIES MANAGEMENT

WBS 1.5.2.3

\begin{tabular}{|c|c|c|c|c|c|c|c|c|c|c|c|c|c|c|c|c|}
\hline $\begin{array}{l}\text { RL } \\
\text { WBS }\end{array}$ & WBS Title & $\begin{array}{l}\text { ADS } \\
\text { Number }\end{array}$ & ADS Title & OCT & NOV & DEC & JAN & FEB & MAR & APR & MAY & JUN & JUL & AUG & SEP & TOTAL \\
\hline 1.5 .2 .3 & $\begin{array}{l}\text { Inventory } \\
\text { Mgmt/Chng }\end{array}$ & $7360-00$ & $\begin{array}{l}\text { Environmental } \\
\text { Support } \\
\text { Inventories } \\
\text { Management }\end{array}$ & 250 & 250 & 250 & 250 & 250 & 250 & 250 & 250 & 250 & 250 & 250 & 251 & 3.001 \\
\hline & & & & & & & & & & & & & & & & \\
\hline & & & & & & & & & & & & & & & & \\
\hline & & & & & & & & & & & & & & & & \\
\hline & & & & & & & & & & & & & & & & \\
\hline & & & & & & & & & & & & & & & & \\
\hline & & & & & & & & & & & & & & & & \\
\hline & & & & & & & & & & & & & & & & \\
\hline & & & TOTAL & 250 & 250 & 250 & 250 & 250 & 250 & 250 & 250 & 250 & 250 & 250 & 251 & 3,001 \\
\hline
\end{tabular}


ENVIRONMENTAL SUPPORT - INVENTORIES MANAGEMENT

FY 1995 MYPP

WBS 1.5.2.3

\begin{tabular}{|c|c|c|c|c|c|c|c|c|c|c|c|c|c|}
\hline Cost Element & ocr & Nov & DEC & JAN & FEB & MAR & APR & MAY & JUN & JUL & AUG & SEP & TOTAL \\
\hline o- Labor & 56 & 56 & 56 & 56 & 56 & 56 & 56 & 56 & 56 & 56 & 56 & 56 & 672 \\
\hline 1 - Material & 89 & 89 & 89 & 89 & 89 & 89 & 89 & 89 & 89 & 89 & 89 & 89 & 1,068 \\
\hline 2 - Purchased Services & 0 & 0 & 0 & 0 & 0 & 0 & 0 & 0 & 0 & 0 & 0 & 0 & 0 \\
\hline 3 - Other Contractors & 0 & 0 & 0 & 0 & 0 & 0 & 0 & 0 & 0 & 0 & 0 & 0 & 0 \\
\hline 4 - Site Services & 56 & 56 & 56 & 56 & 56 & 56 & 56 & 56 & 56 & 56 & 56 & 56 & 672 \\
\hline 5 - Internal Charges & 0 & 0 & 0 & 0 & 0 & 0 & 0 & 0 & 0 & 0 & 0 & 0 & 0 \\
\hline $6 \cdot \operatorname{IRM}$ & 0 & 0 & 0 & 0 & 0 & 0 & 0 & 0 & 0 & 0 & 0 & 1 & 1 \\
\hline 7 - Departmental Overheads & 16 & 16 & 16 & 16 & 16 & 16 & 16 & 16 & 16 & 16 & 16 & 16 & 192 \\
\hline G\&A/CSP & 30 & 30 & 30 & 30 & 30 & 30 & 30 & 30 & 30 & 30 & 30 & 30 & 360 \\
\hline Escalation & 3 & 3 & 3 & 3 & 3 & 3 & 3 & 3 & 3 & 3 & 3 & 3 & 36 \\
\hline & & & & & & & & & & & & & \\
\hline & & & & & & & & & & & & & \\
\hline TOTAL & 250 & 250 & 250 & 250 & 250 & 250 & 250 & 250 & 250 & 250 & 250 & 251 & 3,001 \\
\hline
\end{tabular}




\section{ENVIRONMENTAL SUPPORT - INVENTORIES MANAGEMENT}

\section{FY 1995 MYPP}

WBS 1.5.2.3

\begin{tabular}{|c|c|c|c|c|c|c|c|c|c|c|c|c|c|c|c|c|}
\hline \multicolumn{10}{|c|}{ 3.A.3 Program Funding } & \multirow[b]{2}{*}{ APR } & \multirow[b]{2}{*}{ MAY } & \multirow[b]{2}{*}{ JUN } & \multirow[b]{2}{*}{ JUL } & \multirow[b]{2}{*}{ AUG } & \multirow[b]{2}{*}{ SEP } & \multirow[b]{2}{*}{ TOTAL } \\
\hline $\begin{array}{l}\text { RL } \\
\text { WBS }\end{array}$ & WBS Title & $\begin{array}{l}\text { ADS } \\
\text { Number }\end{array}$ & ADS Title & OCT & NOV & DEC & JAN & FEB & MAR & & & & & & & \\
\hline 1.5.2.3 & $\begin{array}{l}\text { Inventory } \\
\text { Mgmt/Chng }\end{array}$ & $7360-00$ & $\begin{array}{l}\text { Environmental } \\
\text { Support } \\
\text { Inventories } \\
\text { Management }\end{array}$ & 250 & 250 & 250 & 250 & 250 & 250 & 250 & 250 & 250 & 250 & 250 & 251 & 3,001 \\
\hline & & & & & & & & & & & & & & & & \\
\hline & & & & & & & & & & & & & & & & \\
\hline & & & & & & & & & & & & & & & & \\
\hline & & & & & & & & & & & & & & & & \\
\hline & & & & & & & & & & & & & & & & \\
\hline & & & & & & & & & & & & & & & & \\
\hline & & & & & & & & & & & & & & & & \\
\hline & & & TOTAL & 250 & 250 & 250 & 250 & 250 & 250 & 250 & 250 & 250 & 250 & 250 & 251 & 3,001 \\
\hline
\end{tabular}


1.5.2 Environmental Support

U. S. Department of Energy

Richland Operations office

\section{Hanford Filter Test Facility WHC Program Manager}

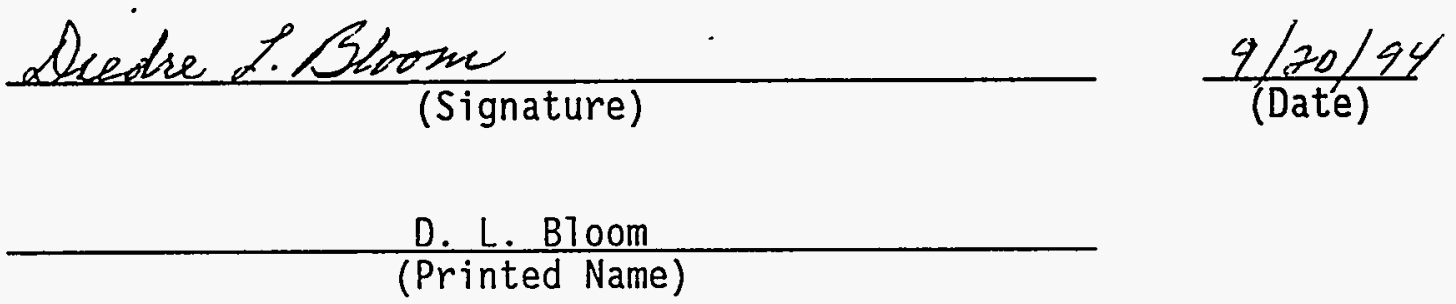




\section{·}


WHC-SP-1112

TABLE OF CONTENTS

1. Program Overview .................. 3-1

1A. Program Vision/Mission .............. 3-2

1B. Program Mission Strategy ............ 3-3 1B1. Technical Function and Requirements ... 3-4 - 3-5 1B2. Program Objectives .......... 3-6

1C. Planning Assumptions ............ . . . 3-7

1D. Issue and Constraints ............. 3-8

2. Program Baseline ............. . . 3-9

2A1. WBS Structure ............ 3-10

2A2. WBS Dictionary ........... . . 3-11

2B. Responsibility Assignment Matrix ......... 3-12

2C. Program Logic Diagram . . . . . . . . . . . 3-13

2D. Program Master Baseline Schedule (PMBS) . . . . . 3-14

$2 E$. Program Performance Baseline Schedule (PPBS) . . . 3-15

2F. Milestone List ............. . . . . . . . . . . . . . .

2G. Milestone Description Sheets . . . . . . . . 3-17

2H. Cost Baseline Summary by Year . . . . . . . . 3-18

2I. Basis of Estimate ............... 3-19

2J. Waste Type Data . . . . . . . 3-20 - 3-26

2K. Planned Staffing (Full-Time Equivalents) . . . . 3-27

2L. Building Blocks ........... 3-28 - 3-29

3. Fiscal Year Work Plan (FYWP) ............ 3- . . . 30

3A1. Cost Estimate Basel ine by Month . . . . . . . . . 3-31

3A2. Program Summary/Cost Element ........... 3-32

3A3. Program Funding . . . . . . . . . . . 3-33 
$\frac{N}{5}$
$\frac{1}{5}$
$\frac{0}{3}$

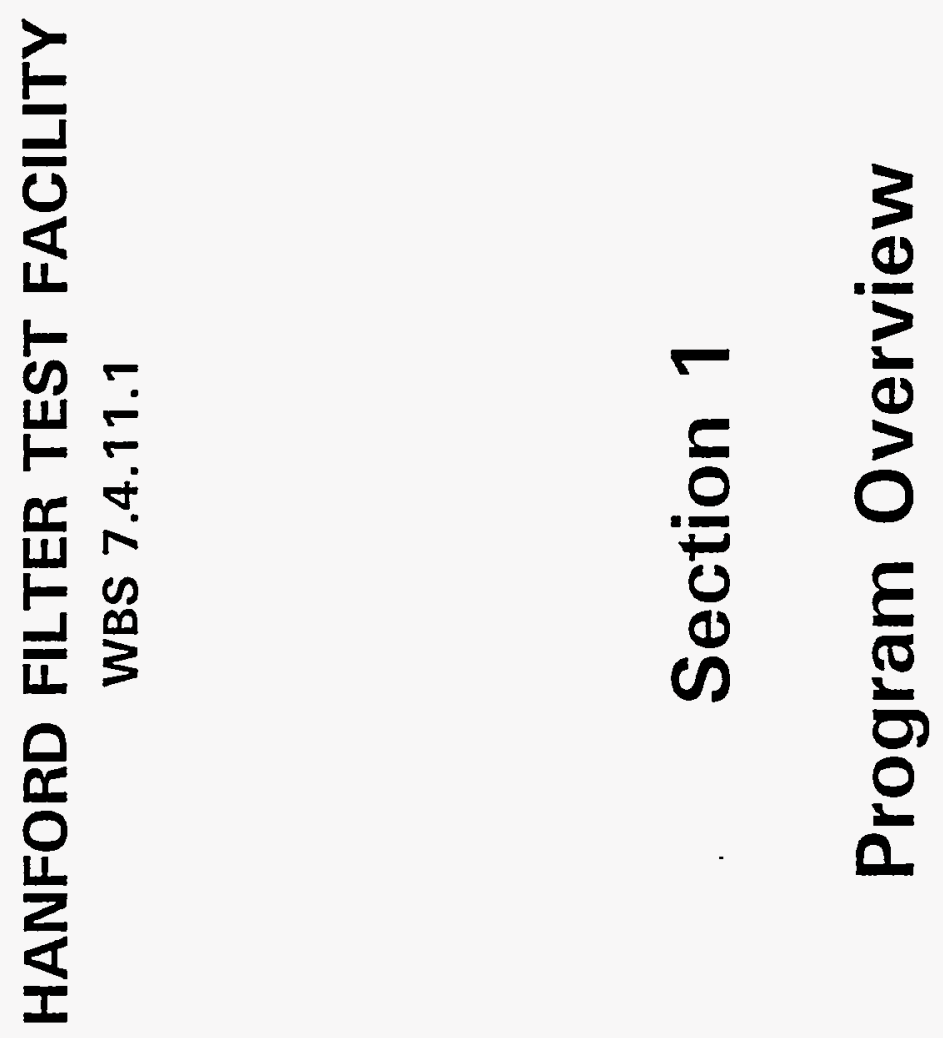

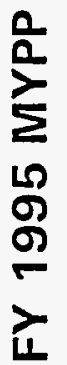




\section{A. Program Vision/Mission}

The Hanford Filter Test Facility (HFTF) provides quality assurance testing and inspection of all High Efficiency Particulate Air (HEPA) filters, HEPA filtered respirator cartridges, and HEPA filtered air movers used by Hanford contractors, other Department of Energy (DOE) contractors, and non-DOE contractors whether on- or off-site. Testing of these filters and cartridges assures environmental compliance and personnel protection for all contractors for whom the service is being provided. The HFTF mission is to continue to test filters and cartridges to assure compliance to technical requirements, and to provide responsive service in order to support the key objectives. 


\section{B. Program Mission Strategy}

1) Support all Westinghouse Hanford Company, Pacific Northwest Laboratory, ICF Kaiser Hanford Company, and Bechtel Hanford, Inc. organizations that depend upon HEPA filters and respirator cartridges to conduct day-to-day operations at Hanford. This function supports the Hanford cleanup mission through regulatory compliance with emission and safety standards.

2) Support other DOE and non-DOE on- or off-site facilities to achieve regulatory compliance with emission and safety standards. 


\section{B1. Technical Function and Requirements}

The technical function and requirements of the HFTF are to assure compliance with specific federal, state and local environmental laws, regulations and agreements.

Applicable Regulatory Statutes

Radiation Protection - Air Emissions, WAC 246-247

Washington Industrial Health and Safety Administration (WISHA) Standard for Asbestos, WAC 296-62-07751, App I

National Emissions Standard for Hazardous Air Pollutants (NESHAPS)

The Resource Conservation and Recovery Act (RCRA) and Amendments

The Comprehensive Environmental Response, Compensation, and Liability Act (CERCLA) and Amendments (SARA) 
FY 1995 MYPP WBS 7.4.11.1

\section{B1. Technical Function and Requirements}

Nuclear Energy Filters (NEF) Standards - NE-F 3-42, -43, -44, and -45

ANSI/ASME N509 - 1989, Nuclear Power Plant Air Cleaning Units and Components

ANSI/ASME N510 - 1989, Testing of Nuclear Air-Cleaning Systems

DOE 6430.1A, General Design Criteria

DOE RLIP 5480.10, Industrial Hygiene Program, Part F., DOE Filter Test Stations and Filter Testing 


\section{B2. Program Objectives}

1) Support all Westinghouse Hanford Company, Pacific Northwest Laboratory, ICF Kaiser Hanford Company, and Bechtel Hanford, Inc. organizations that depend upon HEPA filters and respirator cartridges to conduct day-to-day operations at Hanford. This function supports the Hanford cleanup mission through regulatory compliance with emission and safety standards.

2) Support other DOE and non-DOE on- or off-site facilities to achieve regulatory compliance with emission and safety standards. 


\section{C. Planning Assumptions}

Environmental laws and regulations will continue to change.

Operation of the HFTF is expected to continue until site closure.

Workscope, workload and personnel are expected to remain constant. 


\section{D. Issues and Constraints}

Mil-Std-F51068 has requirements for the acceptance of filters that are not being met by the suppliers for a limited set of filters (e.g., separator, nipple, round). As a result, acceptance of these filters would require additional testing at Rocky Flats or a waiver of the requirement. 
r
$\frac{1}{0}$
0
0
$\frac{1}{3}$

p)

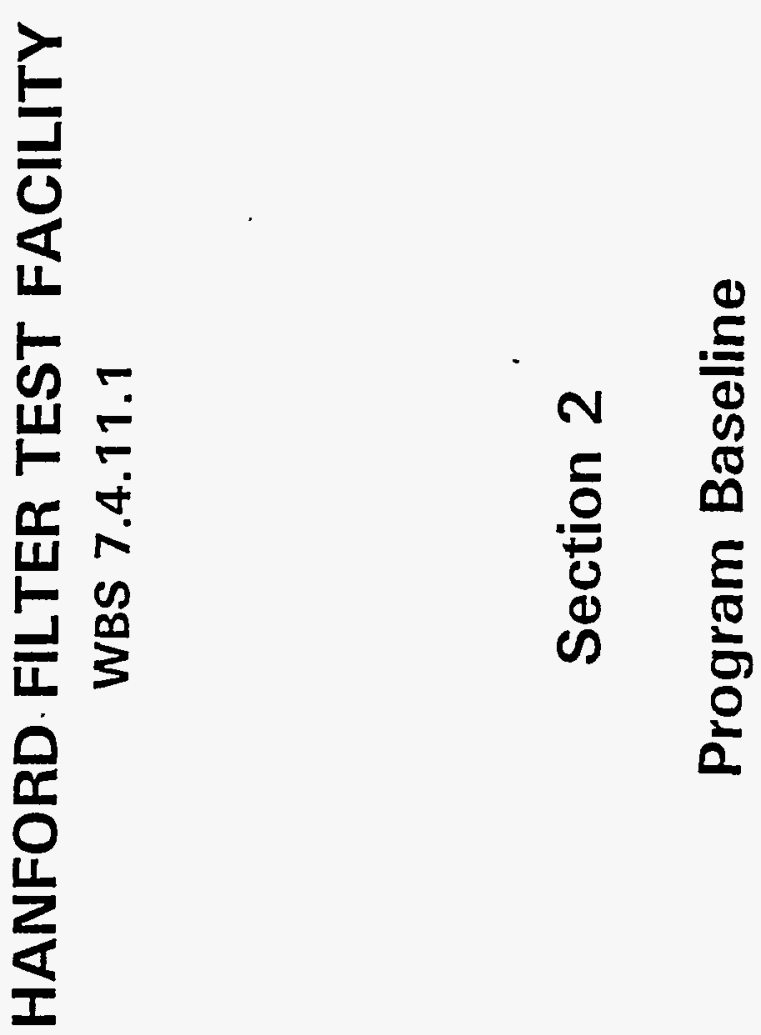

2
$\sum$
2
0
0
0
$\frac{1}{2}$ 


\section{WBS 7.4.11.1}

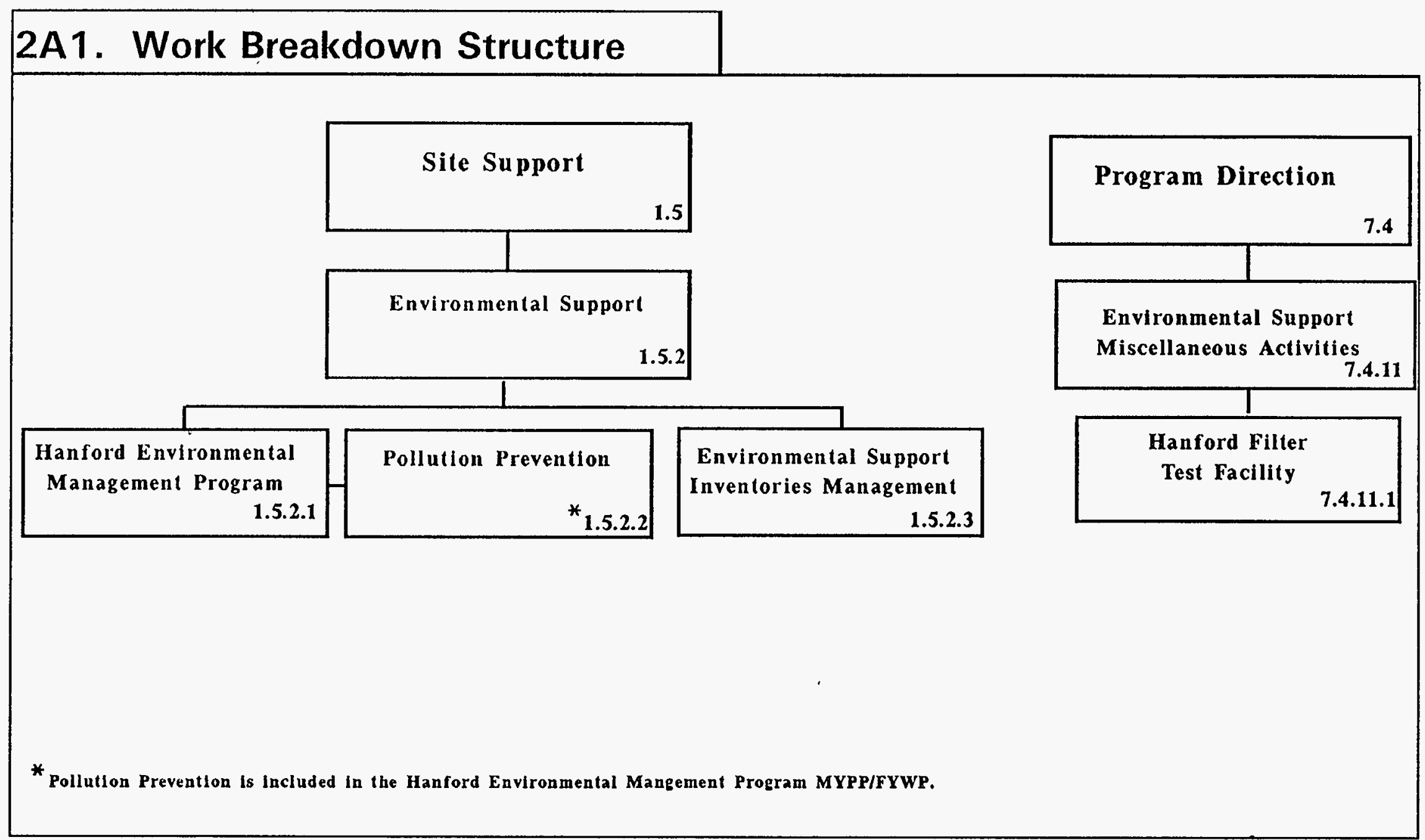




\section{A2. WBS Dictionary}

\section{WORK BREAKDOWN STRUCTURE}

\begin{tabular}{|c|c|c|}
\hline $\begin{array}{l}\text { 1. PROJECT TITLE/PARTICIPANT } \\
\text { Hanford Filter Test Facility }\end{array}$ & September 20,1994 & $\begin{array}{l}\text { 3. DOE-HQ WBS CODE: } \\
3.7 .6 .6\end{array}$ \\
\hline 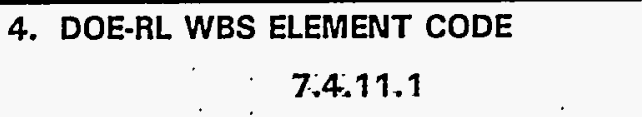 & $\begin{array}{l}\text { 5. WBS ELEMENT TITLE } \\
\text { Hanford Filter Test }\end{array}$ & \\
\hline $\begin{array}{l}\text { 6. CONTRACTOR WBS ELEMENT CODE: } \\
\text { 1R7 }\end{array}$ & $\begin{array}{l}\text { 7. REVISION \# AND AUTHORIZATION } \\
\text { Not Applicable }\end{array}$ & $\begin{array}{l}\text { 8. DATE } \\
\text { Not Applicable }\end{array}$ \\
\hline
\end{tabular}

9. APPROVED CHANGES

10. SYSTEM DESIGN DESCRIPTION NUMBER: TITLE:

\section{BUDGET AND REPORTING NUMBER}

EW-31-10-01-0

12. ELEMENT TASK DESCRIPTION

A. Cost Content

Refer to Section II part $2 \mathrm{H}$ for the Cost Baseline Summary.

B. Technical Content/Work Statement

Provide quality assurance testing and inspection of all HEPA filters, HEPA filtered respirator cartridges, and HEPA filtered air movers used by Hanford contractors, other DOE contractors, and non-DOE contractors whether on- on off- site. Testing of these filters and cartridges assures environmental compliance and personnel protection for all contractors for whom the service is being provided 


\section{HANFORD FILTER TEST FACILITY}

\section{FY 1995 MYPP}

WBS 7.4.11.1

\section{B. Responsibility Assignment Matrix}

\begin{tabular}{|l|l|l|}
\hline PROGRAM ELEMENT & ACTIVITY & COST ACCOUNT \\
\hline
\end{tabular}

7.4.11.1

TITLE

Envi ronmental Support Miscellaneous

Activities

Hanford Filter Test Facility

\begin{tabular}{|l|l|l|l|l|l|}
\hline & 7.4 .11 .1 .1 & & Hanford Filter Test Facility & DL Bloom & Procurement Quality Support \\
\hline & & 7.4 .11 .1 .1 .1 & Hanford Filter Test Facility & DL Bloom & Procurement Quality Support \\
\hline & & & & & \\
\hline & & & & & \\
\hline & & & & & \\
\hline & & & & & \\
\hline
\end{tabular}

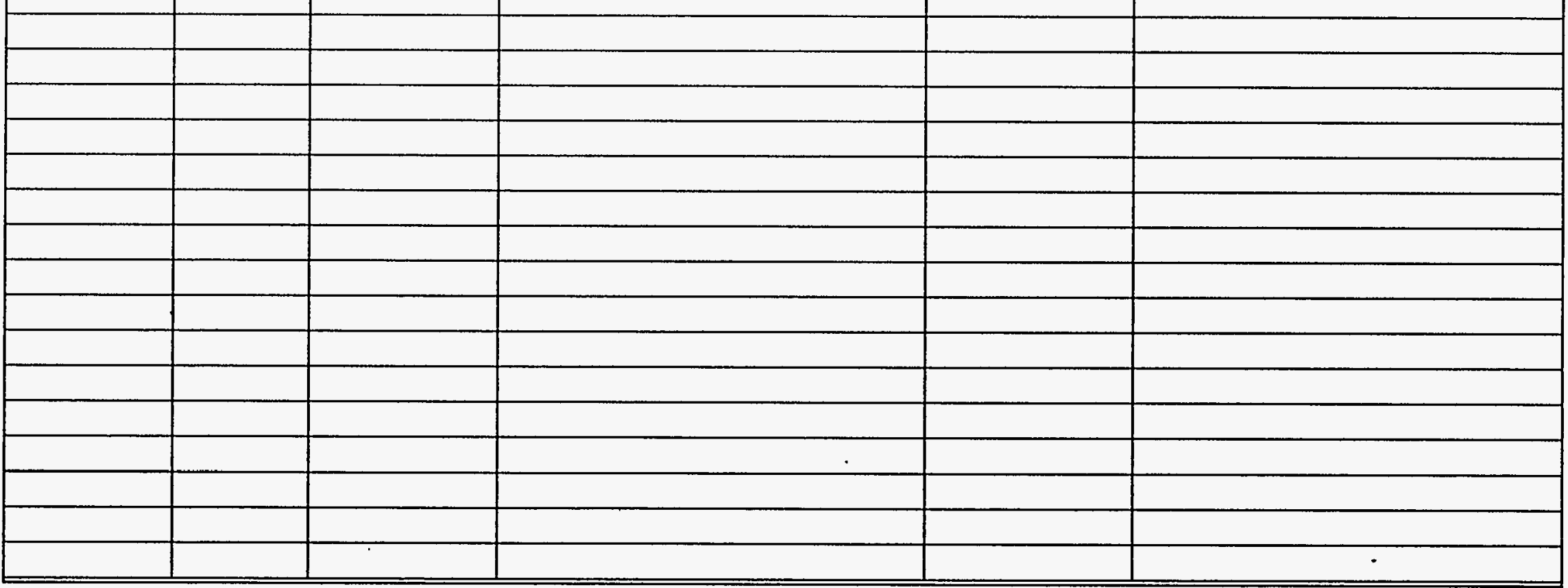




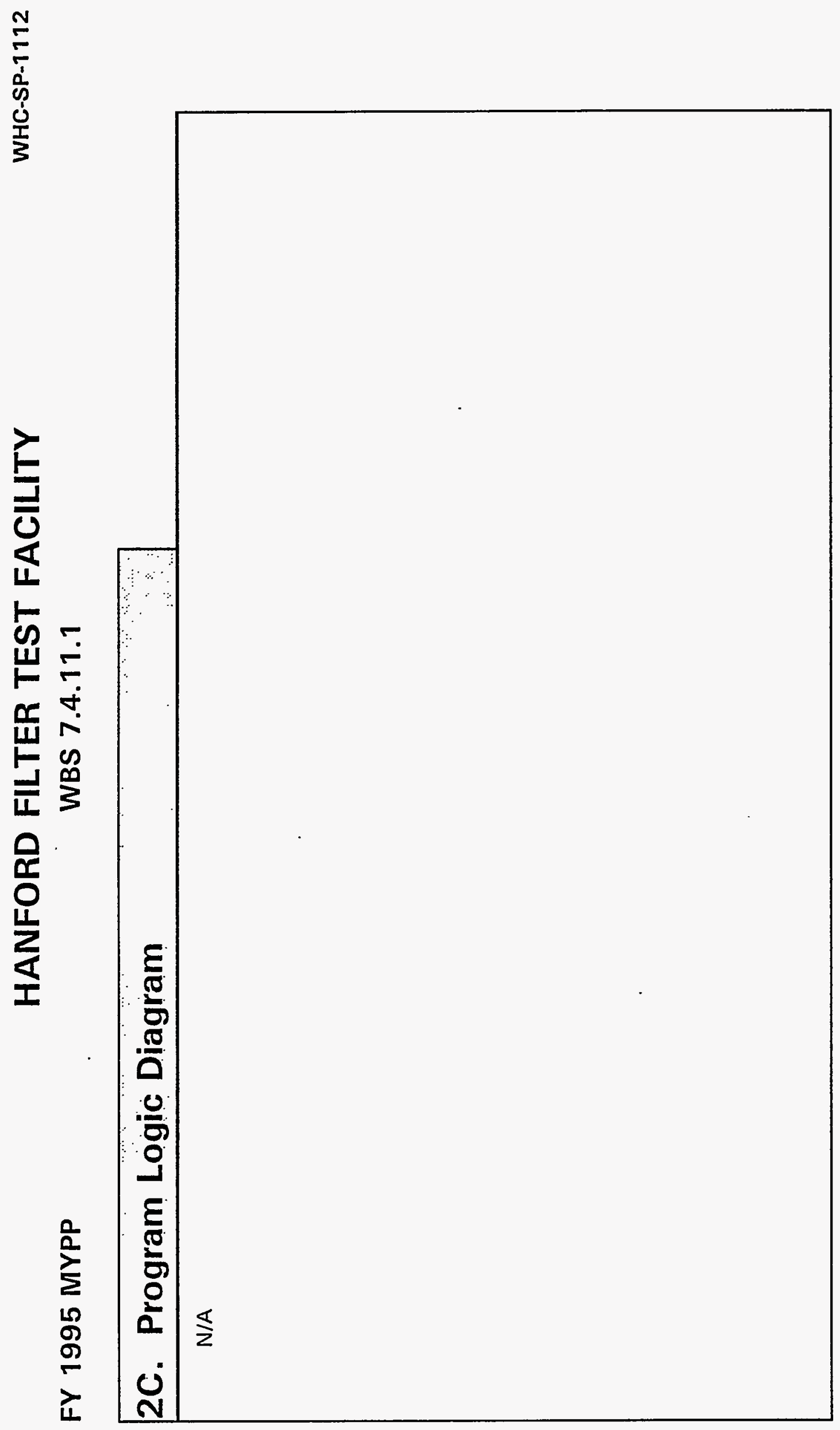




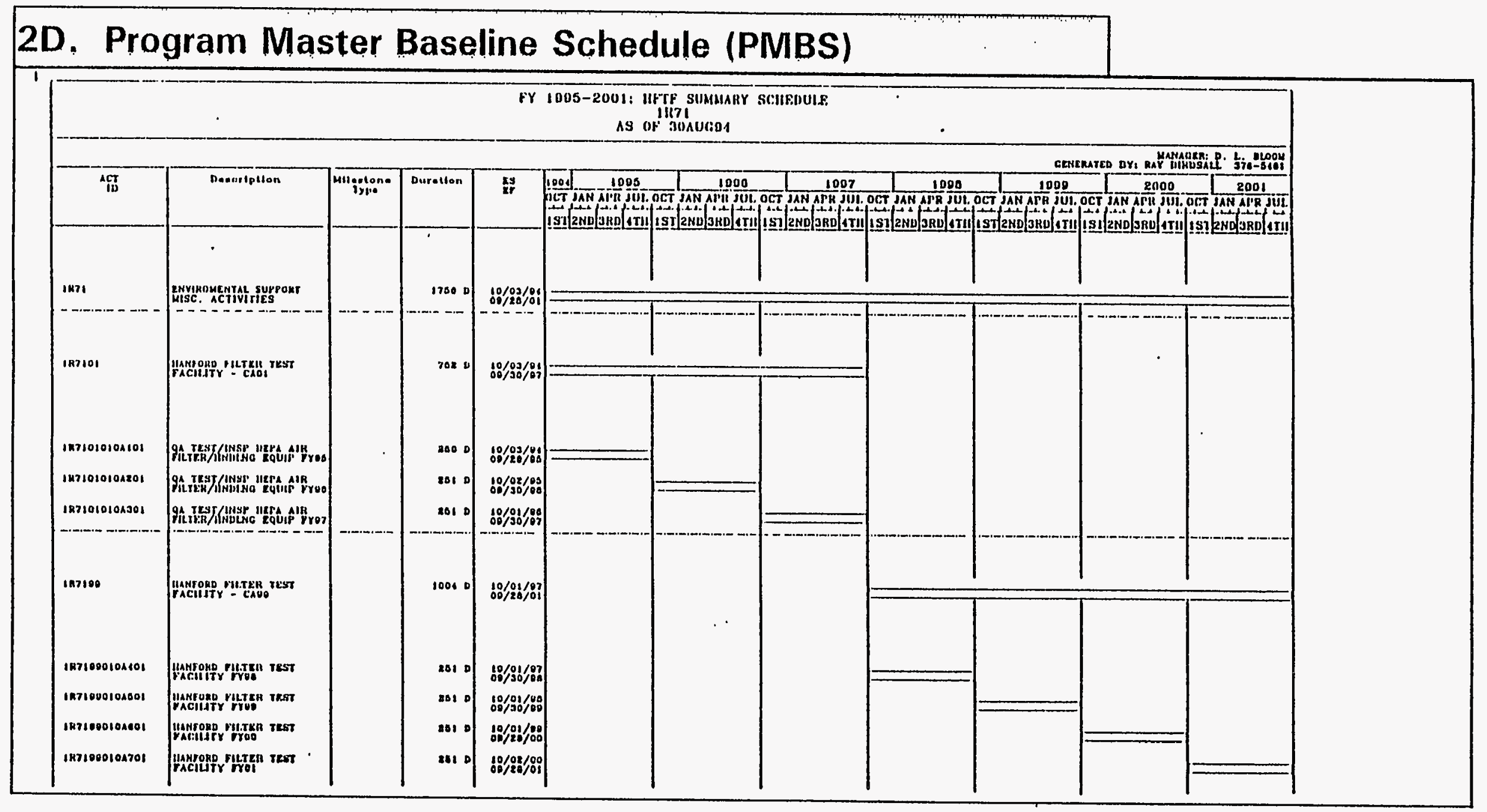




\section{E Program Performance Baseline Schedule (PMBS)}

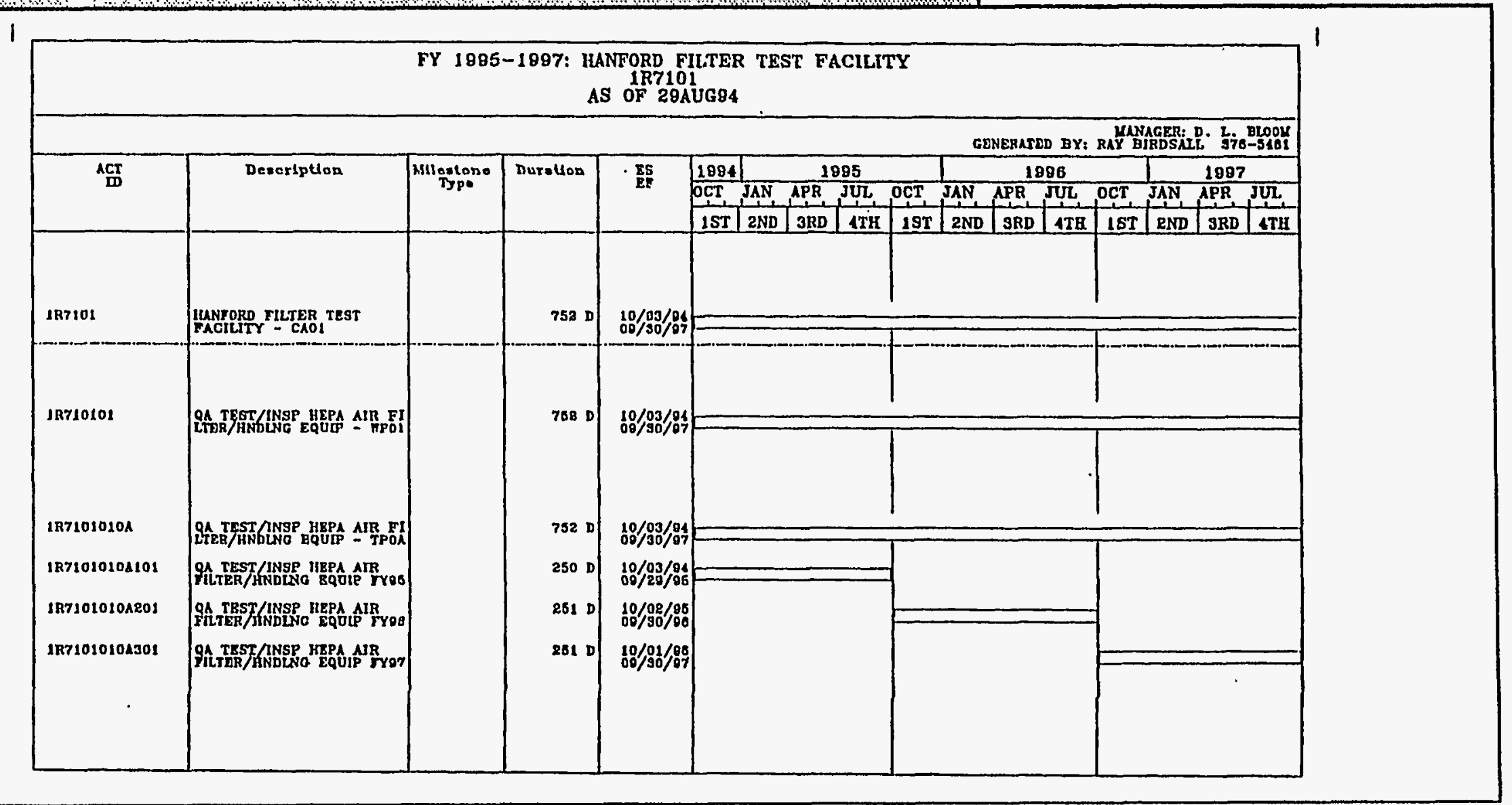




\section{F. Milestone List}

N/A 
WBS 7.4.11.1

\section{G. Milestone Description Sheets}

N/A 


\begin{tabular}{|c|c|c|c|c|c|c|c|c|}
\hline \multicolumn{3}{|c|}{ 2H. Cost Baseline Summary } & \multicolumn{6}{|c|}{ (\$ In Thousands) } \\
\hline Program Element / ADS \# & $\begin{array}{l}\text { Fund Type } \\
\text { (OP, LI, CE, } \\
\text { GPP, ADS) }\end{array}$ & $\begin{array}{l}\text { FY } 1995 \text { by } \\
\text { SMS Guidance }\end{array}$ & $\begin{array}{l}\text { FY } 1996 \\
\text { Plan }\end{array}$ & $\begin{array}{l}\text { FY } 1997 \\
\text { Plan }\end{array}$ & $\begin{array}{l}\text { FY } 1998 \\
\text { Plan }\end{array}$ & $\begin{array}{l}\text { FY } 1999 \\
\text { Plan }\end{array}$ & $\begin{array}{l}\text { FY } 2000 \\
\text { Plan }\end{array}$ & $\begin{array}{l}\text { FY } 2001 \\
\text { Plan }\end{array}$ \\
\hline \multirow[t]{2}{*}{$7.4 .11 .1 / 6695-00$} & OP & 369 & 380 & 391 & 403 & 415 & 427 & 440 \\
\hline & CE & & & 30 & & & & \\
\hline & & & & & & & & \\
\hline & & & & & & & & \\
\hline & & & & & & & & \\
\hline & & & & & & & & \\
\hline & & & & & & & & \\
\hline \multirow{2}{*}{$\begin{array}{l}\text { Total Program } \\
\text { Cost Summary }\end{array}$} & $O P$ & 369 & 380 & 391 & 403 & 415 & 427 & 440 \\
\hline & $\mathrm{CE}$ & 0 & 0 & 30 & 0 & 0 & 0 & 0 \\
\hline \multicolumn{2}{|l|}{ Grand Total } & 369 & 380 & 421 & 403 & 415 & 427 & 440 \\
\hline
\end{tabular}




\section{HANFORD FILTER TEST FACILITY}

\section{Basis of Estimate}

Cost estimating forms were completed for the Hanford Filter Test Facility and can be found in the 2440 Stevens Center Building in Room 2120. 


\section{FY 1995 MYPP}

HANFORD FILTER TEST FACILITY

WHC-SP-1112

WBS 7.4.11.1

Program: Hanford Filter Test Facility

Quantify waste by appropriate waste quantity (e.g. cubic meters, gallons, kilograms, etc.) for the EXECUTION YEAR - FY 1995 for the following WASTE TYPES for expected waste generation, receipt, transfer, and storage:

\begin{tabular}{|c|c|c|c|c|}
\hline Waste Type & $\begin{array}{c}\text { Waste Generated } \\
\text { (waste unit, e.g., } \\
\text { gallons) }\end{array}$ & $\begin{array}{c}\text { Waste Received } \\
\text { (waste unit, and name of } \\
\text { program received from) }\end{array}$ & $\begin{array}{c}\text { Waste Transferred } \\
\text { (waste unit and name of } \\
\text { program transferred from) }\end{array}$ & $\begin{array}{l}\text { Waste Stored } \\
\text { (waste unit) }\end{array}$ \\
\hline \multicolumn{5}{|l|}{ HLW } \\
\hline \multicolumn{5}{|l|}{ LLW (SOLID) } \\
\hline \multicolumn{5}{|l|}{ LLW (LIQUID) } \\
\hline \multicolumn{5}{|l|}{ LLMW (SOLID) } \\
\hline \multicolumn{5}{|l|}{ LLMW (LIQUID) } \\
\hline \multicolumn{5}{|l|}{ LLW (GTC3) } \\
\hline \multicolumn{5}{|l|}{ TRU } \\
\hline \multicolumn{5}{|l|}{ TRUM } \\
\hline \multicolumn{5}{|l|}{ HAZARDOUS } \\
\hline SANITARY (LANDFILL) & 50 gallons & & & \\
\hline $\begin{array}{c}\text { SANITARY (LANDFILL- } \\
\text { ASBESTOS) }\end{array}$ & & & & . \\
\hline
\end{tabular}


FOR THE EXECUTION YEAR - FY 1995 PROVIDE A BRIEF NARRATIVE FOR EACH OF THE FOLLOWING:

PLANS FOR WASTE STORAGE:

Each year, the Hanford Filter Test Facility generates about 50 gallons of nonhazardous waste. HEHF takes care of the necessary paperwork and the transportation of the waste to the landfill. The plans are to continue this arrangement.

PLANS FOR INTERPROGRAM WASTE TRANSFER AND/OR DISPOSITION:

NA

SUMMARY WASTE TYPE ISSUES:

NA 
HANFORD FILTER TEST FACILITY

Program: Hanford Filter Test Facility

Quantify waste by appropriate waste quantity (e.g. cubic meters, gallons, kilograms, etc.) for FY 1996 for the following WASTE TYPES for expected waste generation, receipt, transfer, and storage:

\section{J. Waste Type Projections - FY 1996}

\begin{tabular}{|c|l|c|c|c|}
\hline Waste Type & $\begin{array}{c}\text { Waste Generated } \\
\text { (waste unit, e.g., } \\
\text { gallons) }\end{array}$ & $\begin{array}{c}\text { Waste Received } \\
\text { (waste unit, and name of } \\
\text { program received from) }\end{array}$ & $\begin{array}{c}\text { Waste Transferred } \\
\text { (waste unit and name of } \\
\text { program transferred from) }\end{array}$ & $\begin{array}{c}\text { Waste Stored } \\
\text { (waste unit) }\end{array}$ \\
\hline HLW & & & & \\
\hline LLW (SOLID) & & & & \\
\hline LLW (LIOUID) & & & & \\
\hline LLMW (SOLID) & & & & \\
\hline LLMW (LIOUID) & & & & \\
\hline LLW (GTC3) & & & & \\
\hline TRU & & & & \\
\hline TRUM & & & & \\
\hline HAZARDOUS & & & & \\
\hline SANITARY (LANDFILL) & 50 gallons & & & \\
\hline SANITARY (LANDFILL- \\
ASBESTOS)
\end{tabular}




\section{HANFORD FILTER TEST FACILITY}

FY 1995 MYPP WBS 7.4.11.1

FOR THE YEAR FY 1996, PROVIDE A BRIEF NARRATIVE FOR EACH OF THE FOLLOWING:

PLANS FOR WASTE STORAGE:

Each year, the Hanford Filter Test Facility generates about 50 gallons of nonhazardous waste. HEHF takes care of the necessary paperwork and the transportation of the waste to the landfill. The plans are to continue this arrangement.

PLANS FOR INTERPROGRAM WASTE TRANSFER AND/OR DISPOSITION:

NA

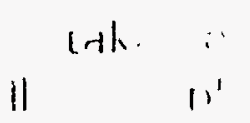

SUMMARY WASTE TYPE ISSUES:

NA 


\section{FY 1995 MYPP}

Program: Hanford Filter Test Facility

Quantify waste by appropriate waste quantity (e.g. cubic meters, gallons, kilograms, etc.) for FY 1997 for the following WASTE TYPES for expected waste generation, receipt, transfer, and storage:

\begin{tabular}{|c|c|c|c|c|}
\hline 2J. Waste Type Projections - FY 1997 & \multicolumn{2}{|c|}{} \\
\hline Waste Type & $\begin{array}{c}\text { Waste Generated } \\
\text { (waste unit, e.g., } \\
\text { gallons) }\end{array}$ & $\begin{array}{c}\text { Waste Received } \\
\text { (waste unit, and name of } \\
\text { program received from) }\end{array}$ & $\begin{array}{c}\text { Waste Transferred } \\
\text { (waste unit and name of } \\
\text { program transferred from) }\end{array}$ & $\begin{array}{c}\text { Waste Stored } \\
\text { (waste unit) }\end{array}$ \\
\hline HLW & & & & \\
\hline LLW (SOLID) & & & & \\
\hline LLW (LIOUID) & & & & \\
\hline LLMW (SOLID) & & & & \\
\hline LLMW (LIOUID) & & & & \\
\hline LLW GTC3 & & & & \\
\hline TRU & & & & \\
\hline TRUM & & & & \\
\hline HAZARDOUS & & & & \\
\hline SANITARY (LANDFILL) & 50 gallons & & & \\
\hline SANITARY (LANDFILL- \\
ASBESTOS)
\end{tabular}


FOR THE YEAR FY 1997, PROVIDE A BRIEF NARRATIVE FOR EACH OF THE FOLLOWING:

PLANS FOR WASTE STORAGE:

Each year, the Hanford Filter Test Facility generates about 50 gallons of nonhazardous waste. HEHF takes care of the necessary paperwork and the transportation of the waste to the landfill. The plans are to continue this arrangement.

PLANS FOR INTERPROGRAM WASTE TRANSFER AND/OR DISPOSITION:

NA

SUMMARY WASTE TYPE ISSUES:

NA 


\section{FY 1995 MYPP}

\section{HANFORD FILTER TEST FACILITY}

Program: Hanford Filter Test Facility

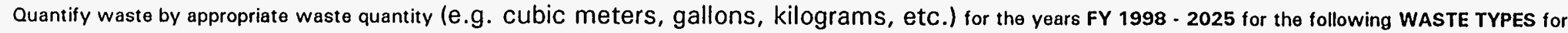
expected waste generation, receipt, transfer, and storage:

\begin{tabular}{|c|c|c|c|c|}
\hline \multicolumn{5}{|c|}{ 2J. Waste Type Projections - FY 1998 - 2025} \\
\hline Waste Type & $\begin{array}{c}\text { Waste Generated } \\
\text { (waste unit, e.g., } \\
\text { gallons) } \\
\end{array}$ & $\begin{array}{l}\text { Waste Received } \\
\text { (waste unit, and name of } \\
\text { program received from) }\end{array}$ & $\begin{array}{l}\text { Waste Transferred } \\
\text { (waste unit and name of } \\
\text { program transferred from) }\end{array}$ & $\begin{array}{l}\text { Waste Stored } \\
\text { (waste unit) }\end{array}$ \\
\hline \multicolumn{5}{|l|}{ HLW } \\
\hline \multicolumn{5}{|l|}{ LLW (SOLID) } \\
\hline \multicolumn{5}{|l|}{ LLW (LIQUID) } \\
\hline \multicolumn{5}{|l|}{ LLMW (SOLID) } \\
\hline \multicolumn{5}{|l|}{ LLMW (LIQUID) } \\
\hline \multicolumn{5}{|l|}{ LLW GTC3 } \\
\hline \multicolumn{5}{|l|}{ TRU } \\
\hline \multicolumn{5}{|l|}{ TRUM } \\
\hline \multicolumn{5}{|l|}{ HAZARDOUS } \\
\hline SANITARY (LANDFILL) & 50 gallons/year & & & \\
\hline $\begin{array}{l}\text { SANITARY (LANDFILL- } \\
\text { ASBESTOS) }\end{array}$ & & & & \\
\hline
\end{tabular}


HANFORD FILTER TEST FACILITY

FY 1995 MYPP WBS 7.4.11.1

\begin{tabular}{|c|c|c|c|c|c|c|c|}
\hline \multicolumn{5}{|c|}{ 2K. Planned Staffing (Full-Time Equivalent) } & & & \\
\hline \multicolumn{8}{|l|}{ JOB FAMIIY } \\
\hline Job category & 1995 & 1996 & 1997 & 1998 & 1999 & 2000 & 2001 \\
\hline MANAGERS & 0.2 & 0.2 & 0.2 & 0.2 & 0.2 & से०: 2 & 0.2 \\
\hline Manager, Exempt & 0.2 & 0.2 & 0.2 & 0.2 & 0.2 & 0.2 & 0.2 \\
\hline ADMIN/OTHER PROFESSIONALS & 1.0 & 1.0 & 1.0 & 1.0 & 1.0 & 1.0 & 1.0 \\
\hline Specialist, Qual Assurance & 1.0 & 1.0 & 1.0 & 1.0 & 1.0 & 1.0 & 1.0 \\
\hline TECHNICIANS & 1.4 & 1.4 & 1.4 & 1.4 & 1.4 & 1.4 & 1.4 \\
\hline Tech, Quality Assurance & 1.4 & 1.4 & 1.4 & 1.4 & 1.4 & 1.4 & 1.4 \\
\hline & & & & \multicolumn{4}{|c|}{ ब. } \\
\hline \multicolumn{8}{|c|}{ TOTALS BY CATEGORTES } \\
\hline EXEMPT & 1.2 & 1.2 & 1.2 & 1.2 & 1.2 & 1.2 & 1.2 \\
\hline NON-EXEMPT NON BARGAINING & 1.4 & 1.4 & 1.4 & 1.4 & 1.4 & 1.4 & 1.4 \\
\hline GRAND TOTALS & 2.6 & 2.6 & 2.6 & 2.6 & 2.6 & 2.6 & 2.6 \\
\hline
\end{tabular}




\begin{tabular}{|c|c|c|c|c|c|c|c|}
\hline 2L. & Building Block & & & FY 1995 & & & $(\$$ in 000$)$ \\
\hline ADS & Description & $\begin{array}{l}\text { RL } \\
\text { Pri }\end{array}$ & $\begin{array}{l}\text { TPA } \\
\text { MS \# }\end{array}$ & $\begin{array}{l}\text { Fund } \\
\text { Type }\end{array}$ & $\begin{array}{c}\text { FY } \\
1995\end{array}$ & $\begin{array}{c}\text { CUM FY } \\
1995\end{array}$ & $\begin{array}{l}\text { Justification of Scope I } \\
\text { Impact if not funded }\end{array}$ \\
\hline $6695-00$ & Hanford Filter Test Facility & A2 & N/A & Expense & 369 & 369 & $\begin{array}{l}\text { Support all Westinghouse Hanford Company, Pacific } \\
\text { Northwest Laboratory, ICF Kaiser Hanford Company, } \\
\text { and Bechtel Hanford, Inc. organizations that depend } \\
\text { upon HEPA filters and respirator cartridges to } \\
\text { conduct day-to-day operations at Hanford. This } \\
\text { function supports the Hanford cleanup mission } \\
\text { through regulatory compliance with emission and } \\
\text { safety standards. } \\
\text { Support other DOE and non-DOE on- or off-site } \\
\text { facilities to achieve regulatory compliance with } \\
\text { emission and safety standards. }\end{array}$ \\
\hline & & & & & & & \\
\hline & & & & & & & \\
\hline & & & & & & & \\
\hline & & & & & & & \\
\hline & & & & & & & \\
\hline & & & & & & & \\
\hline & & & & & & & \\
\hline & & & & & & & \\
\hline
\end{tabular}




\begin{tabular}{|c|c|c|c|c|c|c|c|}
\hline 2L. & Building Blocks & & & FY 1996 & & & $(\$$ in 000$)$ \\
\hline ADS & Description & $\begin{array}{l}\mathrm{RL} \\
\mathrm{Pri}\end{array}$ & $\begin{array}{c}\text { TPA } \\
\text { MS \# }\end{array}$ & $\begin{array}{l}\text { Fund } \\
\text { Type }\end{array}$ & $\begin{array}{l}\text { FY } \\
1996\end{array}$ & $\begin{array}{c}\text { CUM FY } \\
1996\end{array}$ & $\begin{array}{l}\text { Justification of Scope/ } \\
\text { Impactif not funded }\end{array}$ \\
\hline 6695-00 & Hanford Filter Test Facility & A2 & N/A. & Expense & 380 & 380 & $\begin{array}{l}\text { Support all Westinghouse Hanford Company, Pacific } \\
\text { Northwest Laboratory, ICF Kaiser Hanford Company } \\
\text { and Bechtel Hanford, Inc. organizations that depend } \\
\text { upon HEPA filters and respirator cartridges to } \\
\text { conduct day-to-day operations at Hanford. This } \\
\text { function supports the Hanford cleanup mission } \\
\text { through regulatory compliance with emission and } \\
\text { safety standards. } \\
\text { Support other DOE and non-DOE on- or off-site } \\
\text { facilities to achieve regulatory compliance with } \\
\text { emission and safety standards. }\end{array}$ \\
\hline & & & & & & & \\
\hline & & & & & & & \\
\hline & & & & & & & \\
\hline & & & & & & & \\
\hline & & & & & & & \\
\hline & & & & & & & \\
\hline & & & & & & & \\
\hline
\end{tabular}



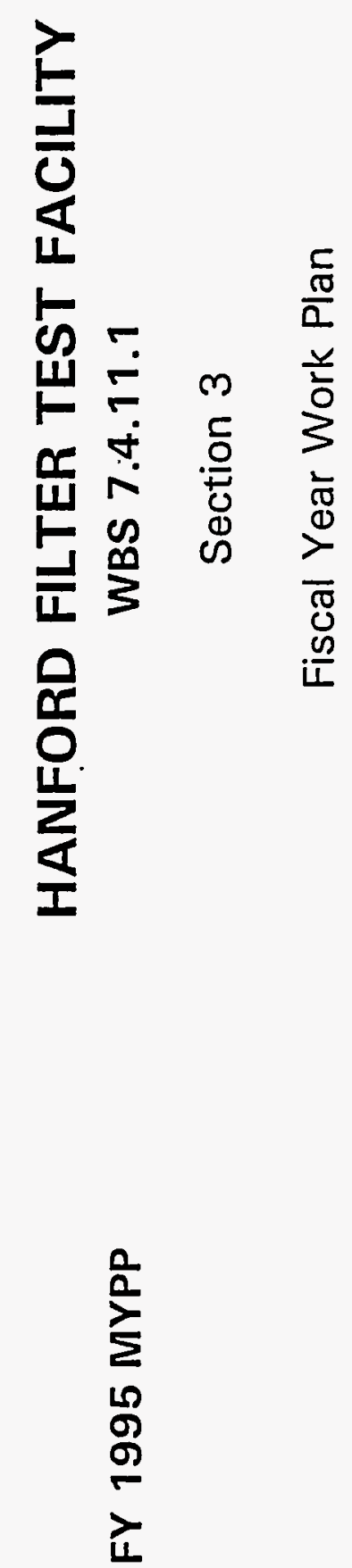
HANFORD FILTER TEST FACILITY .

FY 1995 MYPP WBS 7.4.11.1

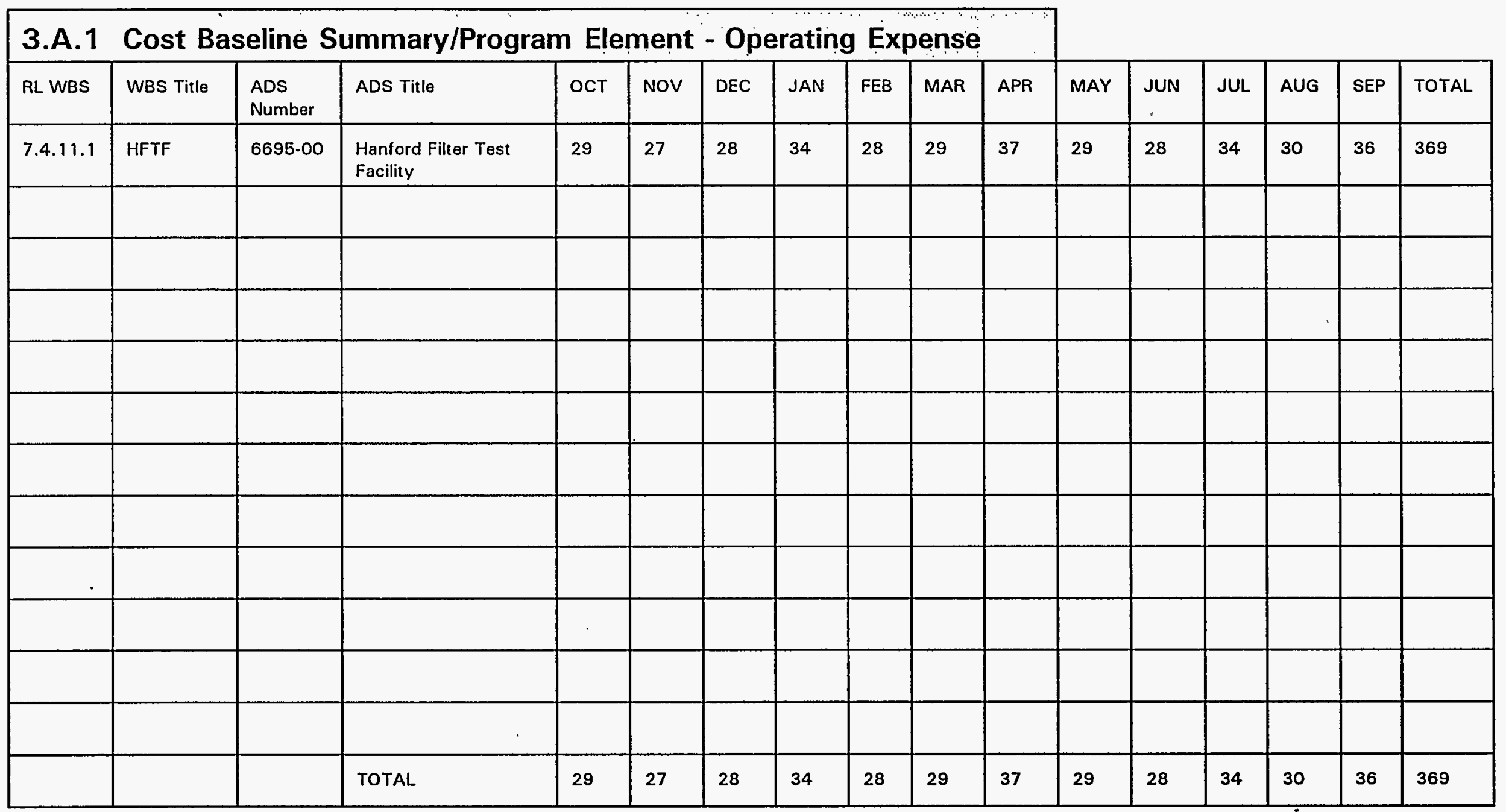


HANFORD FILTER TEST FACILITY

FY 1995 MYPP

WBS 7.4.11.1

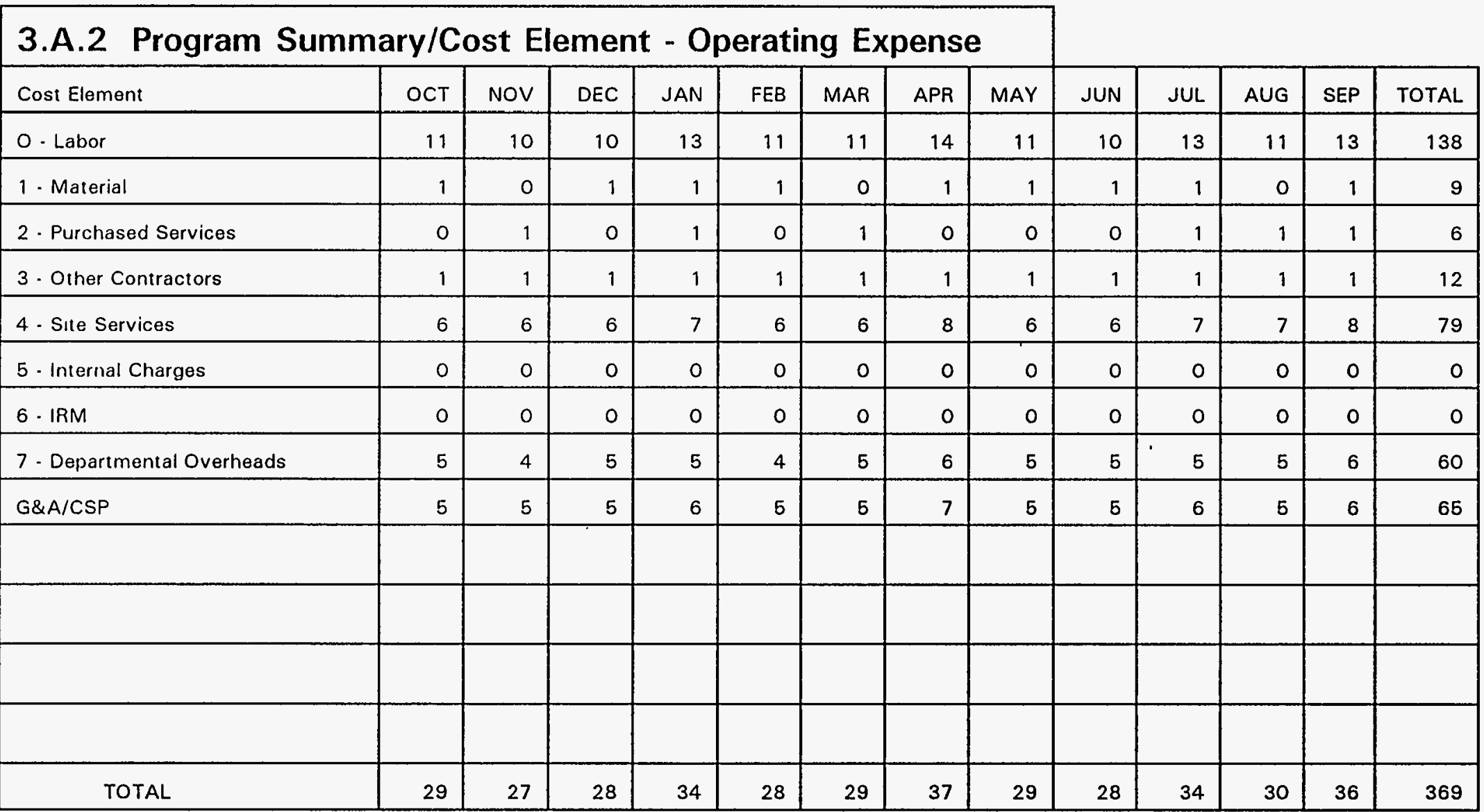


HANFORD FILTER TEST FACILITY

FY 1995 MYPP

WBS 7.4.11.1

\begin{tabular}{|c|c|c|c|c|c|c|c|c|c|c|c|c|c|c|c|c|}
\hline & & Fundin & & & & & & & & & & & & & & \\
\hline RL WBS & WBS Title & $\begin{array}{l}\text { ADS } \\
\text { Number }\end{array}$ & ADS Title & OCT & NOV & DEC & JAN & FEB & MAR & APR & MAY & JUN & JUL & AUG & SEP & TOTAL \\
\hline 7.4.11.1 & HFTF & 6695.00 & $\begin{array}{l}\text { Hanford Filter Test } \\
\text { Facilities }\end{array}$ & 29 & 27 & 28 & 34 & 28 & 29 & 37 & 29 & 28 & 34 & 30 & 36 & 369 \\
\hline & & & & & & & & & & & & & & & & \\
\hline & & & & & & & & & & & & & & & & \\
\hline & & & & & & & & & & & & & & & & \\
\hline & & & & & & & & & & & & & & & & \\
\hline & & & & & & & & & & & & & & & & \\
\hline & & & & & & & & & & & & & & & & \\
\hline & & & & & & & & & & & & & & & & \\
\hline & & & & & & & & & & & & & & & & \\
\hline & & & & & & & & & & & & & & & & \\
\hline & & . & & & & & & & & & & & & & & \\
\hline & & & & & & & & & & & & & & & & \\
\hline & & & TOTAL & 29 & 27 & 28 & 34 & 28 & 29 & 37 & 29 & 28 & 34 & 30 & 36 & 369 \\
\hline
\end{tabular}


Appendix A

Environmental Services

Department Overhead 
WHC-SP-1112

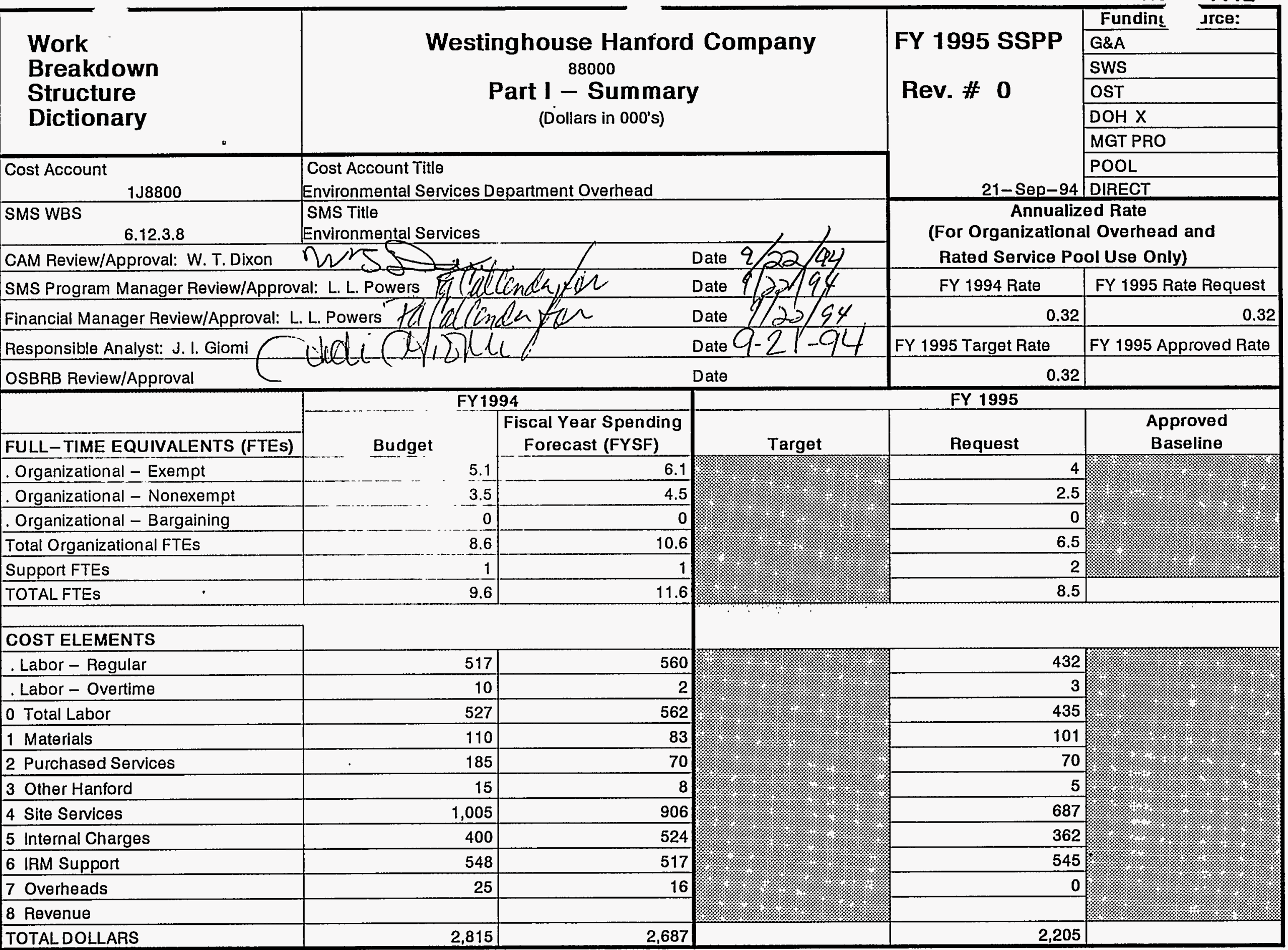




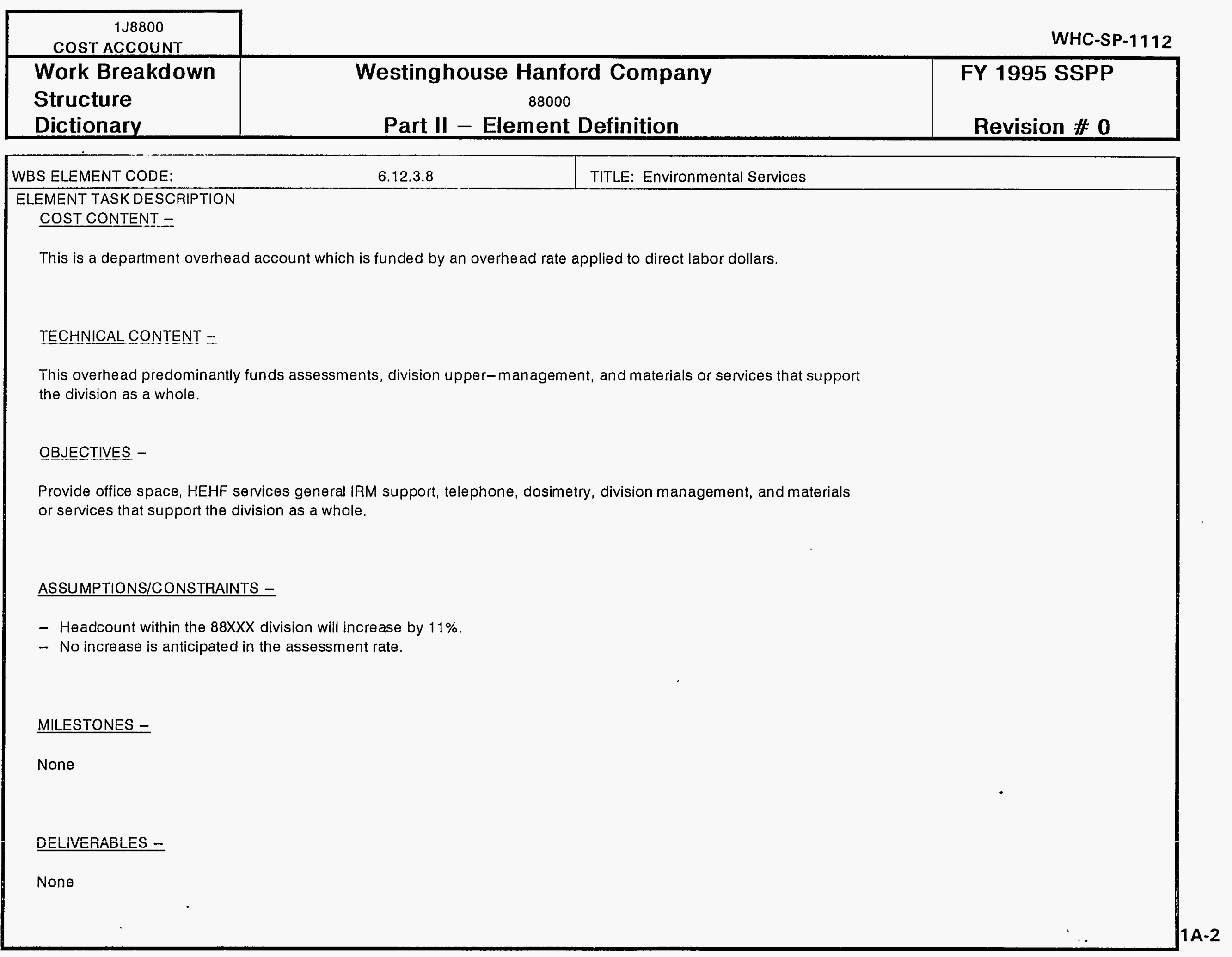


Cost $A_{L}$ junt $1 \mathrm{~J} 8800$

Part : Element Definition (continued)

Wh P-1112

\section{ELEMENT TASK DESCRIPTION}

\section{WORK STATEMENT -}

REQUESTS WITHIN TARGET:

Activity Detailed Description: J880A Environmental Services DOH

This activity provides labor dollars for division Management, and division Secretary. This activity also provides dollars for general

assessments, materials and services.

\section{Activity Detailed Description: J880B Business Management Support}

This activity provides labor dollars for Business Management Manager (.5), Scheduler (.5), two Staff Assistants (1.5), Secretary (.5) and Clerk (1.0).

\section{Activity Detailed Description: J880F 88XXX Financial Support}

Labor Cost

Non-Labor Cost

This activity provides financial analysis support to Environmental Services at the division level.

REQUESTS ABOVE TARGET:

None

Labor Cost Non-Labor Cost

Note: The information on this page for the FYSF is for these three tasks only. Some tasks included in the FY 1994 CAP will not exsist in FY 1995. 


\begin{tabular}{|c|c|c|c|c|c|c|c|c|c|c|c|c|c|c|c|c|c|}
\hline $\begin{array}{l}\text { Work } \\
\text { Breakdown } \\
\text { Structure } \\
\text { Dictionary }\end{array}$ & \multicolumn{14}{|c|}{$\begin{array}{l}\text { Westinghouse Hanford Company } \\
\qquad 88000 \\
\text { Part III - Liquidation Base Analysis } \\
\text { ORGANIZATIONAL OVERHEAD ONLY }\end{array}$} & \multicolumn{3}{|c|}{$\begin{array}{l}\text { FY } 1995 \text { SSPP } \\
\text { Revision \# } 0\end{array}$} \\
\hline $\begin{array}{l}\text { Cost Account } \\
1 \mathrm{~J} 8800 \\
\end{array}$ & \multicolumn{17}{|c|}{$\begin{array}{l}\text { Cost Account Title } \\
\text { Environmental Services Department Overhead }\end{array}$} \\
\hline & 1. & & & & & & $1 \%$ & & & & & & & (1. & $\left(\mathbb{N}_{1}\right)$ & (c) & (a) \\
\hline DIRECT STAFF* & 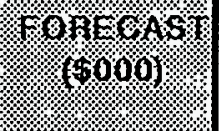 & OCT & Nov & DEC & JAN & FEB & MAR & APR & MAY & JUN & JUL & AUG & SEP & $\begin{array}{c}\text { AVG. } \\
\text { DIRECT } \\
\text { STAFF }\end{array}$ & $\begin{array}{l}\text { AVG. } \\
\text { LABOR } \\
\text { RATE }\end{array}$ & $\begin{array}{c}\text { REALIZATION } \\
\text { HOURS }\end{array}$ & $\begin{array}{l}\text { DIRECT } \\
\text { LABOR } \\
(\$ 000)\end{array}$ \\
\hline EXEMPT & & 114 & 114 & 114 & 114 & 116 & 118 & 120 & 122 & 124 & 126 & 128 & 130 & 120 & 36.68 & 1812 & 7,976 \\
\hline NONEX / PT / TEMP & & 30 & 30 & 30 & 30 & 30 & 30 & 30 & 30 & 37 & 37 & 37 & 30 & 32 & 14.47 & 1812 & 832 \\
\hline OVERTIME FACTOR & & \% & 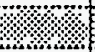 & 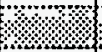 & (a) & (1) & , & 泣: & & & & (4) & & & & & 0 \\
\hline DIRECT LABOR BASE & & 144 & 144 & 144 & 144 & 146 & 148 & 150 & 152 & 161 & 163 & 165 & 160 & 152 & & & 8,808 \\
\hline ORG OVHD COST & 2,687 & 1.9 .1$. & & & & & & & & & & & & & & & 2,205 \\
\hline DIRECT LABOR BASE & 9,057 & (:- & & & & & & & & & & & & & & & 8,808 \\
\hline RATE (COST/BASE) & $29.7 \%$ & & & & & & & & & & & & & & & & $25.0 \%$ \\
\hline
\end{tabular}

Note: Some additional staff changes are expected. We plan to remain within FY 1994 target rate of $32 \%$.

* Direct staff is staff that charges labor to charge codes on which organizational overhead is applied. 
C' ': 1J8800 ENVIRONMENTAL SERVICES DOH

C. ager: DIXON, WT

Resp Org: 88000 ENVIRONMENTAL SERVICES

GL Index:
Funding Source: 1995 EXPENSE

CAP Orig Date : 10/23/92

Budget Analyst: 76 - GIOMI, JUDI

le: UNDISTRIBUTED COSTS

\section{Cap Rev: 00 Cin: Start Completion} Budget (X\$): 2205.0 Date Date

Phone: $6-5792 \quad 10 / 01 / 94 \quad 09 / 30 / 95$

CAP STATUS: D
WORK PACKAGE: 1J8BOOOA ENVIRONMENTAL SERVICES DOH Resp Org: $\quad 88000$ ENVIRONMENTAL SERVICES
Scope of Work:

DOH FOR ENVIRONMENTAL SERVICES DIVISION
Revision No: 00 Date: $08 / 30 / 94$

CAP STATUS: $D$

Task: 1J88000AOA J880A ENVIRONMENTAL SERVICES DOH Responsible Org: 88000 ENVIRONMENTAL SERVICES E V Method: 4 LEVEL OF EFFORT METHOO Parent WBS:
Scope of Work:

DOH FOR ENVIRONMENTAL SERVICES DIVISION
Revision No: 00 Date: $08 / 30 / 94$

Cin Num:

CAP STATUS: D

Fiscal Year: 1995

Performing Org: 88000 EXNIRONMENTAL SERVICES

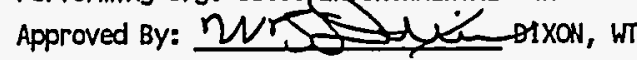

6-0428 Date: 922194

Cost Elements:

Oct Nov Dec

Jan Feb Mar Apr May

Jun Jul Aug Sep Total

\begin{tabular}{|c|c|c|c|c|c|c|c|c|c|c|c|c|c|}
\hline \multirow{3}{*}{ OO NON-EXEMPT } & Staff & 1.0 & 1.0 & 1.0 & 1.0 & 1.0 & 1.0 & 1.0 & 1.0 & 1.0 & 1.0 & 1.0 & 1.0 \\
\hline & Hrs & 148 & 132 & 136 & 163 & 142 & 149 & 183 & 148 & 139 & 156 & 142 & 174 \\
\hline & - $\$$ & 2.1 & 1.9 & 2.0 & 2.4 & 2.1 & 2.2 & 2.6 & 2.1 & 2.0 & 2.3 & 2.1 & 2.5 \\
\hline & Staff & 1.5 & 1.5 & 1.5 & 1.5 & 1.5 & 1.5 & 1.5 & 1.5 & 1.5 & 1.5 & 1.5 & 1.5 \\
\hline APT & Hrs & 222 & 198 & 204 & 245 & 213 & 224 & 275 & 222 & 209 & 234 & 213 & 261 \\
\hline & $\$$ & 8.1 & 7.3 & 7.5 & 9.0 & 7.8 & 8.2 & 10.1 & 8.1 & 7.7 & 8.6 & 7.8 & 9.6 \\
\hline
\end{tabular}

O2 NE OVERTIME HrS

$\$ \quad 0.3$

1111

0.30 .3

10 MATL \& EQUIP

$1.0 \quad 1.0$

14 OFFICE SUPPL

$3.8 \quad 3.8-3.8$

19 COMPUTER-HAR $\$$

\section{$\begin{array}{llll}3.7 & 3.7 & 3.7 & 3.7\end{array}$}

2L. TRAINING-ONS

$1.3 \quad 1.3$

\section{3}

1.3

$11 \quad 11$

$11 \quad 11$

$2 \mathrm{~N}$

2T TRAVEL \& LIV

21 PO CONTRACTS

3D PNL

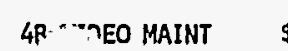

4C MPR

4F OCCUPANCY

$\$ 1.6 \quad 1.6$

\section{$0.6 \quad 0.6$}

0.6

0.3

11
0.3

$11 \quad 11$

11

11

$11 \quad 11 \quad 132$

0.6

$\begin{array}{lll}0.0 & 0.0 & 0.0\end{array}$

0.0

0.0

$1.0 \quad 1$.

$1.0 \quad 1.0$

.01 .0

.01 .0

01.0

0.3

3.0

$\begin{array}{llll}1.5 & 1.5 & 1.5 & 1.5\end{array}$

(1)

1

$\$$

$1.5 \quad 1.5$

$\begin{array}{lllllllllllll}0.4 & 0.4 & 0.4 & 0.4 & 0.4 & 0.4 & 0.4 & 0.4 & 0.4 & 0.4 & 0.4 & 0.4 & 5.0\end{array}$

$\$$

$0.0 \quad 0.0 \quad 0.0$

0.0

$0.0 \quad 0.0 \quad 0.0 \quad 0.0$

$0.0 \quad 0.0$

$0.0 \quad 0.0$

$.0 \quad 0.0$

$0.0 \quad 0.4 \quad 0.4$

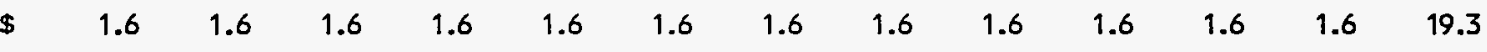

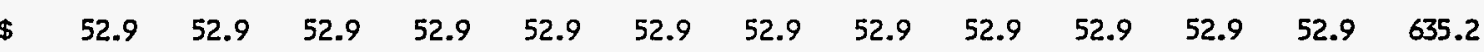




\begin{tabular}{|c|c|c|c|c|c|c|c|c|c|c|c|c|c|c|}
\hline BUS OPERATIO & $\$$ & 1.7 & 1.7 & 1.7 & 1.7 & 1.7 & 1.7 & 1.7 & 1.7 & 1.7 & 1.7 & 1.7 & 1.7 & 20.0 \\
\hline LOCKSMITHS & $\$$ & 0.2 & 0.2 & 0.2 & 0.2 & 0.2 & 0.2 & 0.2 & 0.2 & 0.2 & 0.2 & 0.2 & 0.2 & 2.0 \\
\hline TRANSPORTATI & $\$$ & 0.3 & 0.3 & 0.3 & 0.3 & 0.3 & 0.3 & 0.3 & 0.3 & 0.3 & 0.3 & 0.3 & 0.3 & $4: 0$ \\
\hline FLEET & $\$$ & 0.1 & 0.1 & 0.1 & 0.1 & 0.1 & 0.1 & 0.1 & 0.1 & 0.1 & 0.1 & 0.1 & 0.1 & 1.0 \\
\hline TRNG-SAFE\&EN & $\$$ & 0.0 & 0.0 & 0.0 & 0.0 & 0.0 & 0.0 & 0.0 & 0.0 & 0.0 & 0.0 & 0.0 & 5.0 & 5.0 \\
\hline DOSIMETRY & $\$$ & 13.3 & 13.3 & 13.3 & 13.3 & 13.3 & 13.3 & 13.3 & 13.3 & 13.3 & 13.3 & 13.3 & 13.3 & 160.0 \\
\hline MANAGEMENT P & $\$$ & 9.7 & 9.7 & 9.7 & 9.7 & 9.7 & 9.7 & 9.7 & 9.7 & 9.7 & 9.7 & 9.7 & 9.7 & 116.9 \\
\hline PGM RESERVE & $\$$ & 0.0 & 0.0 & 0.0 & 0.0 & 0.0 & 0.0 & 0.0 & 0.0 & 0.0 & 0.0 & 0.0 & 85.0 & 85.0 \\
\hline CF COMPUTER & $\$$ & 0.3 & 0.3 & 0.3 & 0.3 & 0.3 & 0.3 & 0.3 & 0.3 & 0.3 & 0.3 & 0.3 & 0.3 & 4.0 \\
\hline EUC ASSESSME & $\$$ & 8.9 & 8.9 & 8.9 & 8.9 & 8.9 & 8.9 & 8.9 & 8.9 & 8.9 & 8.9 & 8.9 & 8.9 & 106.8 \\
\hline DOE SUPPORT & $\$$ & 0.1 & 0.1 & 0.1 & 0.1 & 0.1 & 0.1 & 0.1 & 0.1 & 0.1 & 0.1 & 0.1 & 0.1 & 1.0 \\
\hline IRM ADP TRAI & $\$$ & 0.2 & 0.2 & 0.2 & 0.2 & 0.2 & 0.2 & 0.2 & 0.2 & 0.2 & 0.2 & 0.2 & 0.2 & 2.0 \\
\hline IRM MAINT SE & $\$$ & 1.0 & 1.0 & 1.0 & 1.0 & 1.0 & 1.0 & 1.0 & 1.0 & 1.0 & 1.0 & 1.0 & 1.0 & 12.0 \\
\hline NETWORK ACCE & $\$$ & 11.7 & 11.7 & 11.7 & 11.7 & 11.7 & 11.7 & 11.7 & 11.7 & 11.7 & 11.7 & 11.7 & 11.7 & 140.0 \\
\hline MULTIMEDIA S & $\$$ & 1.3 & 1.3 & 1.3 & 1.3 & 1.3 & 1.3 & 1.3 & 1.3 & 1.3 & 1.3 & 1.3 & 1.3 & 15.0 \\
\hline TELEPHONE SE & $\$$ & 22.0 & 22.0 & 22.0 & 22.0 & 22.0 & 22.0 & 22.0 & 22.0 & 22.0 & 22.0 & 22.0 & 22.0 & 264.0 \\
\hline ORG OVERHEAD & & 0.0 & 0.0 & 0.0 & 0.0 & 0.0 & 0.0 & 0.0 & 0.0 & 0.0 & 0.0 & 0.0 & 0.0 & 0.0 \\
\hline \multirow[t]{3}{*}{ Total BCwS } & Staff & 2.5 & 2.5 & 2.5 & 2.5 & 2.5 & 2.5 & 2.5 & 2.5 & 2.5 & 2.5 & 2.5 & 2.5 & 2.5 \\
\hline & Hrs & 381 & 341 & 351 & 419 & 366 & 384 & 469 & 381 & 359 & 401 & 366 & 446 & 4664 \\
\hline & $\$$ & 149.4 & 148.3 & 148.6 & 150.5 & 149.0 & 149.5 & 151.9 & 149.4 & 150.3 & 151.5 & 150.5 & 249.1 & 1898.3 \\
\hline
\end{tabular}

otal BCWP:

ask: 1J88000AOB J880B PROJECT SERVICES DOH BUDGET lesponsible Org: 88000 ENVIRONMENTAL SERVICES $\checkmark$ Method: 4 LEVEL OF EFFORT METHOD arent WBS:
Scope of Work: DOH FOR PROJECT SERVICES
Revision No: 00 Date: 09/21/94

Cin Num: CAP STATUS: D

Fiscal Year: 1995

erforming Org: gopo RROJECT SERYICES

pproved By: 2 Q 6-9558 Date: I I

:ost Elements. Foct Nov Dec Jan feb Mar Apr May Jun Jul Aug Sep Total

\begin{tabular}{|c|c|c|c|c|c|c|c|c|c|c|c|c|c|}
\hline Staff & 1.5 & 1.5 & 1.5 & 1.5 & 1.5 & 1.5 & 1.5 & 1.5 & 1.5 & 1.5 & 1.5 & 1.5 & 1.5 \\
\hline Hrs & 222 & 198 & 204 & 245 & 213 & 224 & 275 & 222 & 209 & 234 & 213 & 261 & 2720 \\
\hline$\$$ & 3.2 & 2.9 & 3.0 & 3.5 & 3.1 & 3.2 & 4.0 & 3.2 & 3.0 & 3.4 & 3.1 & 3.8 & 39.4 \\
\hline
\end{tabular}




\begin{tabular}{|c|c|c|c|c|c|c|c|c|c|c|c|c|c|c|c|}
\hline & & Staff & 2.5 & 2.5 & 2.5 & 2.5 & 2.5 & 2.5 & 2.5 & 2.5 & 2.5 & 2.5 & 2.5 & 2.5 & 2.5 \\
\hline \multirow[t]{2}{*}{ c } & MPT & Hrs & 370 & 330 & 340 & 408 & 355 & 373 & 458 & 370 & 348 & 390 & 355 & 435 & 4532 \\
\hline & & $\$$ & 13.6 & 12.1 & 12.5 & 15.0 & 13.0 & 13.7 & 16.8 & 13.6 & 12.8 & 14.3 & 13.0 & 16.0 & 166.2 \\
\hline \multicolumn{3}{|c|}{ 7C ORG OVERHEAD } & 0.0 & 0.0 & 0.0 & 0.0 & 0.0 & 0.0 & 0.0 & 0.0 & 0.0 & 0.0 & 0.0 & 0.0 & 0.0 \\
\hline \multirow{3}{*}{\multicolumn{2}{|c|}{ Total BCWS }} & Staff & 4.0 & 4.0 & 4.0 & 4.0 & 4.0 & 4.0 & 4.0 & 4.0 & 4.0 & 4.0 & 4.0 & 4.0 & 4.0 \\
\hline & & Hrs & 592 & 528 & 544 & 653 & 568 & 597 & 733 & 592 & 557 & 624 & 568 & 696 & 7252 \\
\hline & & $\$$ & 16.8 & 15.0 & 15.4 & 18.5 & 16.1 & 16.9 & 20.8 & 16.8 & 15.8 & 17.7 & 16.1 & 19.7 & 205.6 \\
\hline
\end{tabular}

Total BCWP:

Task: 1J88000AOF J880F 88XXX FINANCIAL SUPPORT Responsible Org: 88000 ENVIRONMENTAL SERVICES E V Method: 4 LEVEL OF EFFORT METHCO Parent WBS:
Scope of Work:

Revision No: 00 FINANCIAL SUPPORT OF ENVIRONMENTAL SERVICES DIVISI Date: 07/28/94 ON

CAP STATUS: $D$

Fiscal Year: 1995

Performing Org:-8Go90/PRogam gontroL

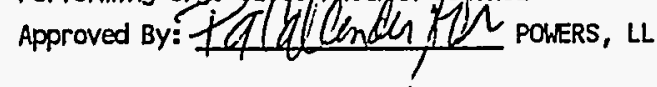
2-3283 Date: / /

Cost Elements:

Staff oct Nov Dec

Jan Feb Mar Apr May

Hrs

$0.5 \quad 0.5$

$0.5 \quad 0.5$

OO NON-EXEMPT

$\$$

1.4

$\begin{array}{ll}0.5 & 0.5\end{array}$

.50 .5

.5

0.5

0.5

50.5

Jun Jul

Aug Sep Total

Staff $\quad 1.5$

01 EXEMPT

Hrs 22

1.51 .5

$1.5 \quad 1.5$

222

$198 \quad 20$

$.5 \quad 1.5$

$1.5 \quad 1.5$

$1.5 \quad 1.5$

$\begin{array}{lll}198 & 204 & 245\end{array}$

213

1.5
224
6.9

$\begin{array}{rr}.5 & 1.5 \\ 24 & 2 \\ 6 & 8.5\end{array}$

1.7

1.4

0.5
70

0.5

$\begin{array}{lll}0.5 & 0.5 & 0.5\end{array}$

7C ORG OVERHEAD

$$
0.0 \quad 0.0 \quad 0.0
$$

$\begin{array}{llll}0.0 & 0.0 & 0.0 & 0.0\end{array}$

$.5 \quad 1.5$

1.51 .5

$1.5 \quad 1.5$

$\begin{array}{llll}1.5 & 1.5 & 1.5 & 1.5\end{array}$

$\begin{array}{lllllll}175 & 222 & 209 & 234 & 213 & 261 & 2720\end{array}$

\begin{tabular}{llllllll}
\hline .5 & 6.8 & 6.4 & 7.2 & 6.6 & 8.0 & 83.8
\end{tabular}

\begin{tabular}{|c|c|c|c|c|c|c|c|c|c|c|c|c|c|c|}
\hline \multirow[t]{3}{*}{ Total BCWS } & Staff & 2.0 & 2.0 & 2.0 & 2.0 & 2.0 & 2.0 & 2.0 & 2.0 & 2.0 & 2.0 & 2.0 & 2.0 & 2.0 \\
\hline & Hrs & 296 & 264 & 272 & 327 & 284 & 299 & 367 & 296 & 279 & 312 & 284 & 348 & 3628 \\
\hline & $\$$ & 8.2 & 7.3 & 7.6 & 9.1 & 7.9 & 8.3 & 10.2 & 8.2 & 7.8 & 8.7 & 7.9 & 9.7 & 100.9 \\
\hline
\end{tabular}

Total BCWP:

Total wp

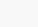

\begin{tabular}{|c|c|c|c|c|c|c|c|c|c|c|c|c|c|c|}
\hline \multirow{3}{*}{ NON-EXEMPT } & Staff & 3.0 & 3.0 & 3.0 & 3.0 & 3.0 & 3.0 & 3.0 & 3.0 & 3.0 & 3.0 & 3.0 & 3.0 & 3.0 \\
\hline & Hrs & 444 & 396 & 408 & 490 & 426 & 448 & 550 & 444 & 418 & 468 & 426 & 522 & 5440 \\
\hline & $\$$ & 6.8 & 6.0 & 6.2 & 7.5 & 6.5 & 6.8 & 8.4 & 6.8 & 6.4 & 7.1 & 6.5 & 7.9 & 82.7 \\
\hline
\end{tabular}

$\begin{array}{rrrrrrrrrrrrrrr} & \text { Staff } & 5.5 & 5.5 & 5.5 & 5.5 & 5.5 & 5.5 & 5.5 & 5.5 & 5.5 & 5.5 & 5.5 & 5.5 & 5.5 \\ 01 \text { EXEMPT } & \text { Hrs } & 814 & 726 & 748 & 898 & 781 & 821 & 1008 & 814 & 766 & 858 & 781 & 957 & 9972 \\ & \$ & 28.5 & 25.5 & 26.2 & 31.5 & 27.4 & 28.8 & 35.4 & 28.5 & 26.9 & 30.1 & 27.4 & 33.6 & 349.7\end{array}$

\begin{tabular}{|c|c|c|c|c|c|c|c|c|c|c|c|c|c|c|}
\hline O2 MIF OVERTIME & Hrs & 11 & 11 & 11 & 11 & 11 & 11 & 11 & 11 & 11 & 11 & 11 & 11 & 132 \\
\hline & $\$$ & 0.3 & 0.3 & 0.3 & 0.3 & 0.3 & 0.3 & 0.3 & 0.3 & 0.3 & 0.3 & 0.3 & 0.3 & 3.0 \\
\hline 10 MATL \& EQUIP & $\$$ & 1.0 & 1.0 & 1.0 & 1.0 & 1.0 & 1.0 & 1.0 & 1.0 & 1.0 & 1.0 & 1.0 & 1.0 & 12.0 \\
\hline 14 OFFICE SUPPL & $\$$ & 3.8 & 3.8 & 3.8 & 3.8 & 3.8 & 3.8 & 3.8 & 3.8 & 3.8 & 3.8 & 3.8 & 3.8 & 45.0 \\
\hline
\end{tabular}


19 COMPUTER-HAF

2L TRAINING-ONS

WM TRAINING-OFF

2N ED REIMBURSE

2T TRAVELL \& LIV :

21 PO CONTRACTS

3D PNL.

4B VIDEO MAINT

4C MPR

4F OCCUPANCY

$\$$

$3.7 \quad 3.7$

$\begin{array}{llllll}1.3 & 1.3 & 1.3 & 1.3 & 1.3 & 1.3\end{array}$

$0.6 \quad 0.6$

$\begin{array}{llll}0.6 & 0.6 & 0.6 & 0.6\end{array}$

$0.0 \quad 0.0 \quad 0.0$

$0.0 \quad 0$

$\begin{array}{llll}1.5 & 1.5 & 1.5 & 1.5\end{array}$

.51 .5

$\begin{array}{llll}1.5 & 1.5 & 1.5 & 1.5\end{array}$

.51

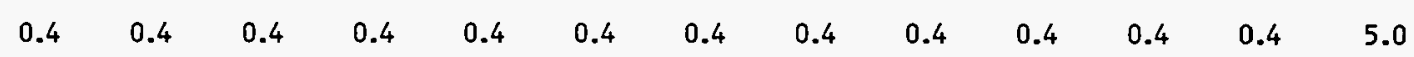

\begin{abstract}
$0.0 \quad 0.0 \quad 0.0$
\end{abstract}

\begin{abstract}
$1.6 \quad 1.6 \quad 1.6$
\end{abstract}
$52.9 \quad 52.9 \quad 52.9$

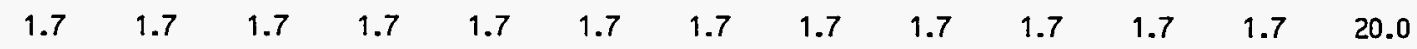

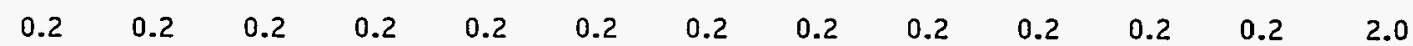

4N LOCKSMITHS

4P TRANSPORTATI

\&V FLEET

41 TRNG-SAFE\&EN

SD DOSIMETRY

$\begin{array}{lllllll}0.3 & 0.3 & 0.3 & 0.3 & 0.3 & 0.3 & 0.3\end{array}$

$0.1 \quad 0.1$

$0.1 \quad 0.1$

0.1

$\begin{array}{llll}0.1 & 0.1 & 0.1 & 0.1\end{array}$

$0.0 \quad 0$.

$0.0 \quad 0.0$

$\begin{array}{llllll}13.3 & 13.3 & 13.3 & 13.3 & 13.3 & 13.3\end{array}$

$\begin{array}{lllll}9.7 & 9.7 & 9.7 & 9.7 & 9.7^{\circ}\end{array}$

$\begin{array}{llllll}0.0 & 0.0 & 0.0 & 0.0 & 0.0 & 0.0\end{array}$

$\begin{array}{llll}0.3 & 0.3 & 0.3 & 0.3\end{array}$

0.3

\begin{abstract}
$\begin{array}{llll}8.9 & 8.9 & 8.9 & 8.9\end{array}$
\end{abstract}
8.9

\begin{abstract}
$\begin{array}{lllll}0.1 & 0.1 & 0.1 & 0.1 & 0.1\end{array}$
\end{abstract}
10

$\begin{array}{llllll}0.2 & 0.2 & 0.2 & 0.2 & 0.2 & 0.2\end{array}$

$\begin{array}{llllll}1.0 & 1.0 & 1.0 & 1.0 & 1.0 & 1.0\end{array}$

$\begin{array}{lllllll}11.7 & 11.7 & 11.7 & 11.7 & 11.7 & 11.7 & 11.7\end{array}$

$11.7 \quad 11.7$

$\begin{array}{llllll}11.7 & 11.7 & 11.7 & 11.7 & 11.7 & 140.0\end{array}$

SP MULTIMEDIA S :

\begin{abstract}
$\begin{array}{lllll}1.3 & 1.3 & 1.3 & 1.3 & 1.3\end{array}$
\end{abstract}
$1.3 \quad 1$

$22.0 \quad 22.0 \quad 22.0 \quad 22.0 \quad 22$.

$\begin{array}{llll}0.3 & 0.3 & 0.3 & 4.0\end{array}$

ST TELEPHONE SE

7C ORG OVERHEAD

$\begin{array}{llllll}0.0 & 0.0 & 0.0 & 0.0 & 0.0 & 0.0\end{array}$

0.0

$\begin{array}{lllllllllllll}8.5 & 8.5 & 8.5 & 8.5 & 8.5 & 8.5 & 8.5 & 8.5 & 8.5 & 8.5 & 8.5 & 8.5 & 8.5\end{array}$ 
WHC-SP-1112

FDS-B620-R

Financial Data System

09/21/94 19:29

COST ACCOUNT PLAN - 1J8800 - VERSION 1995

Page: 5

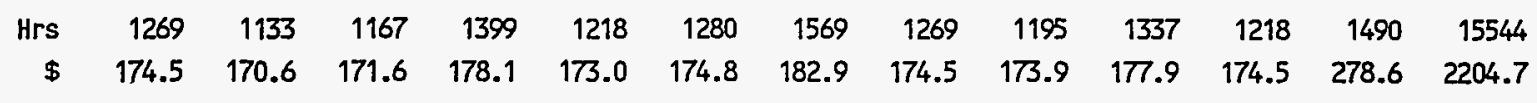

Total GA/CSP

$0.0 \quad 0.0$

0.0

0.0

$0.0 \quad 0.0 \quad 0.0$

0.0

0.0

0.0

0.0

0.0

0.0

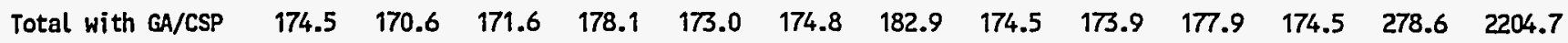

Approval Signatures:

Function Mgr:

Cap Status

D

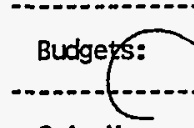

C.A. Mgr:

1A-9 